\title{
Multiscale and Multiphysics Computational Frameworks for
} Nano- and Bio-Systems

\author{
Thesis by \\ Hyungjun Kim \\ In Partial Fulfillment of the Requirements \\ for the Degree of \\ Doctor of Philosophy
}

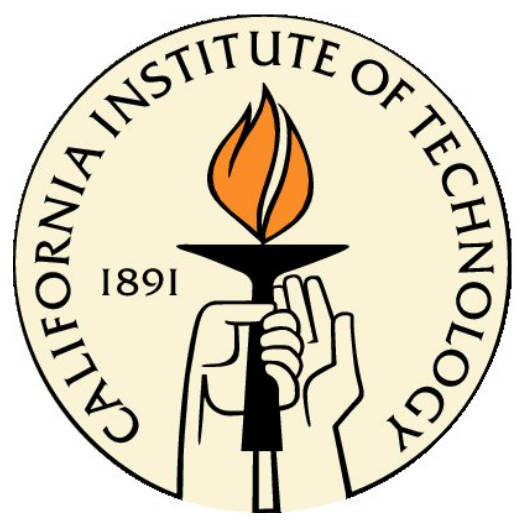

California Institute of Technology

Pasadena, California

2009

(Defended May 4, 2009) 
(c) 2009

Hyungjun Kim

All Rights Reserved 
I dedicate this $\mathrm{PhD}$ thesis to God and my loving parents. 


\section{Acknowledgments}

I would like to express my gratitude to Prof. Bill Goddard, who guided me to become an independent researcher during my $\mathrm{PhD}$ journey. He allowed me the freedom to spend my time on many different projects, trained me to solve various problems, and let me have a broad scientific scope with sharp instinct and creative ideas. His wide range of research interests and enthusiasm for science always provided plenty of insights and thoughts, which, I believe will be a great asset to me as I continue my research life.

Prof. Seung Soon Jang, at Georgia Institute of Technology, taught me a lot of simulation techniques and tips, along with his systematic way of thinking to tackle scientific problems, during his days at Caltech. Even after he moved to Gatech, he continues to be my mentor about science and non-science, and I really appreciate his invaluable advice.

I also appreciate Prof. Eok Kyun Lee, who was my undergraduate advisor. He introduced me to computational and theoretical chemistry, and furthermore, he first taught me how to figure out and solve science problems. I am always indebted to him for his warm encouragement and support.

My committee members, Prof. Vincent McKoy, Prof. Mark Davis, Prof. Jack Beauchamp, and Prof. Jim Heath, have provided useful insights and guidance through the candidacy, proposal, and thesis defense. Moreover, it has been an honor for me to work on several projects with them.

I also thank Dr. Isik Kanik and Dr. Luther Beegle at Jet Propulsion Laboratory (JPL) for supporting me during the IMS project. Their fruitful discussions helped me successfully complete our project. It was a great thrill for me to have a chance to collaborate with NASA.

While working at the MSC, it was a pleasure to have many friends and colleagues who shared a similar interest. I want to thank all MSC members, especially, Sergey Zybin and Julius Su who helped me a lot on the energetic material project and the eFF project with their kind advice. I would also like to thank my friend, Mu-Jeng Cheng, for engaging in scientific discussions as well as listening to my random complaints. I owe a lot of coffees to Jiyoung Heo, Sang Soo Han and Soo-Kyung Kim, too.

Finally, I wish to thank Hugh Kim and Young Shik Shin, who gave me many insights on the experiments while conducting our lipid projects. And last but not least, I am truly grateful for all the scientific, mental, and sometimes physical help from all other friends at KAIST and Caltech. 


\section{Abstract}

Multiscale and multiphysics simulation strategy is important to investigate complex problems in nature because it provides a systematic method to understand underpinning physics of the systems depending on the size. In this thesis, we discuss how such multiscale and multiphysics simulation framework can explain and rationalize the experimental observations in several nano- and biosystems. Furthermore, we exhibit the computational simulation methods that play major roles to rationally design novel materials with desired properties in next generation nano electronic devices, alternative energy materials, life science, and so on.

Chapter 1 reviews the significance of multiscale and multiphysics simulation strategy. In this chapter, we briefly discuss the multiscale and multiphysics natures in nano- and bio-systems, and detailed examples are contained in the next chapters. Chapter 2 introduces an electric field induced conformational change mechanism, which is responsible for the unique current-voltage $(I-V)$ behavior of nano device, negative differential resistance (NDR). In Chapter 3, the on/off kinetics of the Stoddart-Heath rotaxane-based programmable molecular electronic switch is discussed in terms of the free energy quantities. Chapter 4 explores sodium diffusion through the aluminum-doped zeolite BEA system, and the effect of water uptake amount is thoroughly discussed. This has importance for the application of zeolite to proton exchange membranes for fuel cells (PEMFC). In Chapters 5 and 6 , the ion mobilities of tertiary and quaternary ammonium cations (precursors for lipids), and phosphatidylcholine (PC) lipid cations are investigated, respectively. In order to compute the ion mobilities of the precursors and entire lipids, we develop a modified trajectory (TJ) method dealing with the complicated integrals of interaction terms. QM and MD simulations are performed to determine the structures and charge distributions. In Chapter 7, we study how the model lung system of lipid monolayer with surfactant protein B (SP-B) responds to ozone introduction. In parallel with the field induced droplet ionization (FIDI) mass spectrometry study, MD simulations identify the distinct ozone reaction mechanism at the interface, and the role of SP-B at the pulmonary surfactant (PS) system on the oxidative stresses.

From these studies, we suggest various multiscale and multiphysics modeling approaches depending on the characteristics of systems and objectives. These efforts allow us to overcome the limited time- and length-scales of the monoscale simulations. In addition, we expect that an establishment 
of such multiscale modeling procedures will invoke interdisciplinary studies by tightly combining the developments occurring independently across fields. 


\section{Contents}

Acknowledgments $\quad$ iv

Abstract $\quad$ v

1 Introduction 1

2 Negative Differential Resistance of Oligo (Phenylene Ethynylene) Self-Assembled Monolayer Systems: The Electric Field Induced Conformational Change Mechanism $\quad 8$

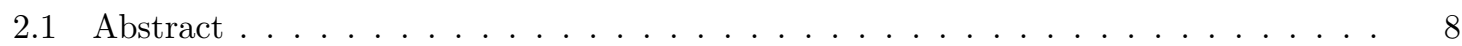

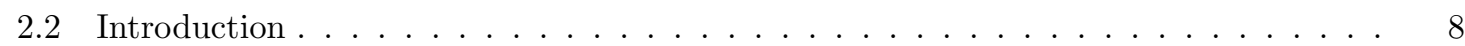

2.3 Simulation Details . . . . . . . . . . . . . . . . . . . . . . 10

2.3.1 Computational Details of QM Calculations . . . . . . . . . . . . 10

2.3.2 Conductivities of $\mathrm{P}$ and T Conformations (NEGF Calculations) . . . . . . 10

2.3.3 Coarse-Grained NN Interacting Hamiltonian ～. . . . . . . . . . . . . . 10

2.3.4 Extracting NN Model Parameters from QM/FF Energies . . . . . . . . . . . 11

2.3.5 Coarse-Grained MC Simulations . . . . . . . . . . . . . . . . . . . . 13

2.4 Results and Discussion . . . . . . . . . . . . . . . . . . . . . . . . . 13

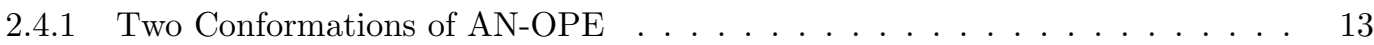

2.4 .2 Electrical Conductivities of $\mathrm{P}$ and $\mathrm{T} \ldots \ldots \ldots \ldots \ldots$

2.4.3 Response to Constant External Field . . . . . . . . . . . . . . . . . . . . . . 21

2.4.4 NDR for Time Dependent Electric Field . . . . . . . . . . . . . . . . . . . 24

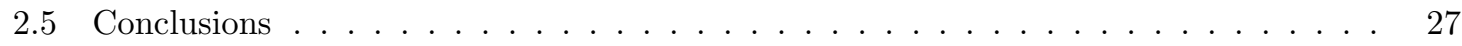

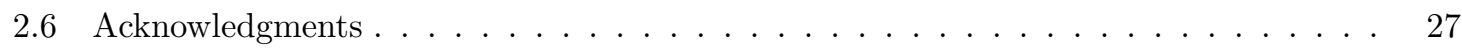

$\begin{array}{lr}\text { Bibliography } & 28\end{array}$

3 Free Energy Barrier for Molecular Motions in Bistable [2/Rotaxane Molecular Electronic Devices $\quad 30$

$3.1 \quad$ Abstract . . . . . . . . . . . . . . . . . . . . . . 30 
3.2 Introduction . . . . . . . . . . . . . . . . . . . . . 31

3.3 Simulation Details . . . . . . . . . . . . . . . . . . . 33

3.3.1 Potential of Mean Force from Constrained Molecular Dynamics Simulation . 33

3.3.2 Constrained Molecular Dynamics Simulation _. . . . . . . . . . . . 35

3.3.3 Force Field and MD Parameters . . . . . . . . . . . . . . . . . 36

3.4 Results and Discussion . . . . . . . . . . . . . . . . . . . 38

3.4.1 Charge Scheme: Adiabatic Approximation . . . . . . . . . . . . . . 38

3.4 .2 Free Energy Profiles from PMF Calculations . . . . . . . . . . . . . . . . 39

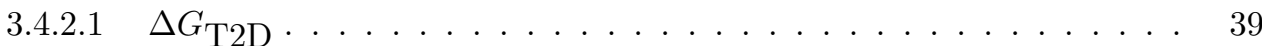

3.4.2.2 $\Delta G_{\mathrm{T} 2 \mathrm{D}}^{\ddagger}$ and $\Delta G_{\mathrm{D} 2 \mathrm{~T}}^{\ddagger}$ for Neutral Case . . . . . . . . . 41

3.4.2.3 $\Delta G_{\mathrm{T} 2 \mathrm{D}}^{\ddagger}$ and $\Delta G_{\mathrm{D} 2 \mathrm{~T}}^{\ddagger}$ for Oxidized Cases . . . . . . . . . 42

3.4.2.4 Effect of Coulombic Energy and van der Waals Energy . . . . . . . 42

3.5 Conclusions . . . . . . . . . . . . . . . . . . . 45

3.6 Acknowledgments . . . . . . . . . . . . . . . . . . . 46

$\begin{array}{lr}\text { Bibliography } & 47\end{array}$

4 Sodium Diffusion through Aluminum-Doped Zeolite BEA System: Effect of Water Solvation

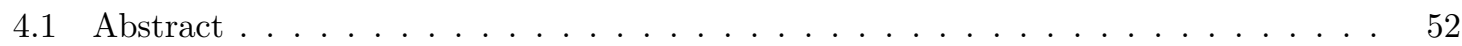

4.2 Introduction . . . . . . . . . . . . . . . . . . . . . 52

4.3 Simulation Details . . . . . . . . . . . . . . . . . . . . 55

4.3 .1 Force Field . . . . . . . . . . . . . . . . . . . . 55

4.3.2 Grand Canonical Monte Carlo (GCMC) method and Molecular Dynamics (MD) Simulation . . . . . . . . . . . . . . . . . . . 5 55

4.3.3 Construction of Models and Calculation of Properties . . . . . . . . . 56

4.4 Results and Discussion . . . . . . . . . . . . . . . . . . . 56

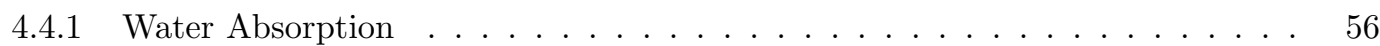

4.4 Structure of Water in Zeolite . . . . . . . . . . . . . . . . 58

4.4.3 Effect of Water Contents on Sodium Diffusion . . . . . . . . . . . . . . . 60

4.4.4 Effect of Temperature on Sodium Diffusion . . . . . . . . . . . . . 63

4.5 Conclusions . . . . . . . . . . . . . . . . . . . . . 65

4.6 Acknowledgments . . . . . . . . . . . . . . . . . . 68

$\begin{array}{lr}\text { Bibliography } & 69\end{array}$ 
5 Experimental and Theoretical Investigation into the Correlation between Mass and Ion Mobility for Choline and Other Ammonium Cations in $\mathrm{N}_{2} \quad 72$

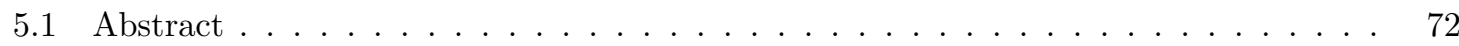

5.2 Introduction . . . . . . . . . . . . . . . . . . . . 73

5.3 Experimental Section . . . . . . . . . . . . . . . . . . 75

5.3.1 Chemicals and Reagents . . . . . . . . . . . . . . 75

5.3.2 Electrospray Ionization Ion Mobility Spectrometer . . . . . . . . . . . . 75

5.3 .3 Computational Modeling _.................... 76

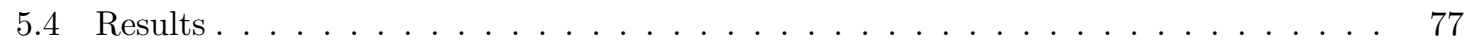

5.4.1 Mass-Mobility Correlation of Ammonium Cations . . . . . . . . . . . 77

5.4.2 Tertiary and Quaternary Ammonium Cations with Similar Molecular Weights 79

5.4.3 Functional Group Isomers of Ammonium Cations . . . . . . . . . . . . . . . 81

5.4.4 Collision Cross-Sections of Ions in $\mathrm{N}_{2}$ via the Trajectory Method . . . . . . 81

5.5 Discussion . . . . . . . . . . . . . . . . . . . . . 83

5.5.1 Classical Ion-Neutral Collision Model _. . . . . . . . . . . . . . . 83

5.5.2 Computational Trajectory Method . . . . . . . . . . . . . . . 84

5.5.3 Ion-Quadrupole Potential . . . . . . . . . . . . . . . . . . 84

5.5 .4 Ion-Induced Dipole Potential . . . . . . . . . . . . . . . . . 84

5.5 .5 Van der Waals Potential . . . . . . . . . . . . . . . . . . . 86

5.5.6 Mass-Mobility Correlation . . . . . . . . . . . . . . . . 86

5.6 Conclusions . . . . . . . . . . . . . . . . . . . . . . 89

5.7 Acknowledgments . . . . . . . . . . . . . . . . . . . . . . . 90

$\begin{array}{lr}\text { Bibliography } & 91\end{array}$

6 Structural Characterization of Unsaturated Phospholipids Using Traveling Wave $\begin{array}{ll}\text { Ion Mobility Spectrometry } & 94\end{array}$

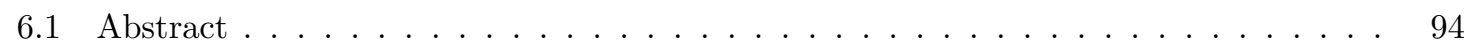

6.2 Introduction . . . . . . . . . . . . . . . . . . . . . 95

6.3 Experimental Section . . . . . . . . . . . . . . . . . . 96

6.3.1 Chemicals and Reagents . . . . . . . . . . . . . 96

6.3.2 Electrospray Ionization Traveling Wave Ion Mobility Mass Spectrometer . . . 97

6.3.3 Collision Cross-Section Calibration . . . . . . . . . . . . . . . 97

6.3.4 Computational Modeling . . . . . . . . . . . . . . . 99

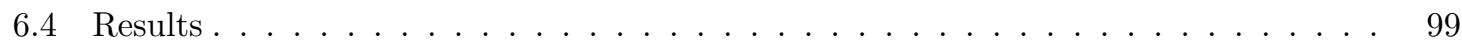

6.4.1 Saturated Phosphatidylcholine Cations . . . . . . . . . . . . . . 99

6.4.2 Unsaturated Phosphatidylcholine Cations . . . . . . . . . . . . . 101 
6.4.3 Sodiated Phosphatidylcholine Cations . . . . . . . . . . . . . . . 101

6.4.4 Estimated Collision Cross-Sections of Ions Using T-Wave Calibration . . . . 103

6.4.5 Determination of Collision Cross-Sections of Ions . . . . . . . . . . . . . . . . 104

6.4.6 Calculated Collision Cross-Sections of Ions Using the Trajectory Method . . . 106

6.5 Discussion . . . . . . . . . . . . . . . . . . . 106

6.5.1 Effect of Drift Gas on Ion Mobility . . . . . . . . . . . . . 106

6.5.2 Geometrical Effect on the Collision Cross-Sections of Phosphatidylcholine Cations . . . . . . . . . . . . . . . . . . . . 108

6.5.3 Mass-Mobility Correlations of Phophatidylcholine Cations . . . . . . . . . . . 109

6.5.4 Characterizing Unsaturated Phosphatidylcholines from Mass-Mobility Corre-

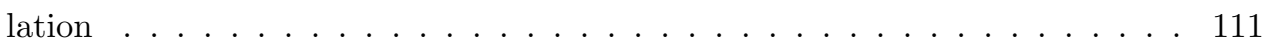

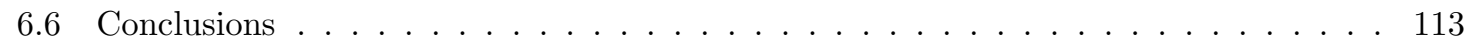

6.7 Acknowledgments . . . . . . . . . . . . . . . . . . . 115

$\begin{array}{lr}\text { Bibliography } & 116\end{array}$

7 Interfacial Reactions of Ozone with Lipids and Proteins in a Model Lung Surfactant System

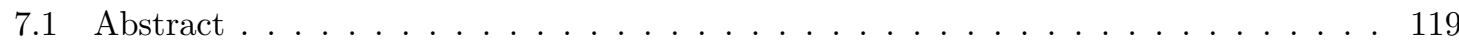

7.2 Introduction . . . . . . . . . . . . . . . . . . . . 120

7.3 Methods . . . . . . . . . . . . . . . . . . . . . . 121

7.3.1 Chemicals and Reagents . . . . . . . . . . . . . . . 121

7.3.2 Online FIDI-MS Technique and Heterogeneous Oxidation by $\mathrm{O}_{3} \ldots \ldots 121$

7.3.3 Molecular Dynamic Simulations . . . . . . . . . . . . . . . . . . 123

7.4 Results and Discussion . . . . . . . . . . . . . . . . . . . 125

7.4.1 Interfacial Reaction of POPG with $\mathrm{O}_{3} \ldots \ldots \ldots \ldots$

7.4 .2 Interfacial Oxidation of SP-B $\mathrm{B}_{1-25} \ldots \ldots \ldots \ldots \ldots$

7.4.3 Oxidation of $\mathrm{SP}_{-1-25}$ in POG Monolayer by $\mathrm{O}_{3}$. . . . . . . . . . . . . . . 128

7.4 .4 Interactions of SP-B I-25 $_{1}$ in a Lipid Monolayer . . . . . . . . . . . . . . 128

7.5 Conclusions . . . . . . . . . . . . . . . . . . . . . 132

7.6 Acknowledgments . . . . . . . . . . . . . . . . . . . . 134

Bibliography 135

A Discussions on Coarse-Graining of Time- and Length-Scale in Monte Carlo Sim$\begin{array}{ll}\text { ulations for AN-OPE SAM } & 138\end{array}$

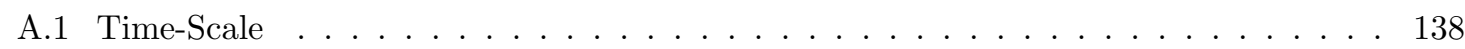

A.2 Length-Scale . . . . . . . . . . . . . . . . . . . . . . 138 
B Effect of Molecular Fluctuations on the Electrical Conductivity of AN-OPE SAM140

C NDR in Other OPE-derivative Systems 143

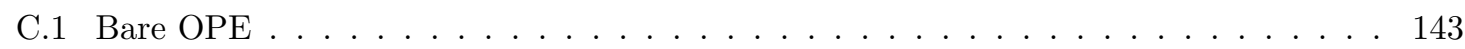

C.2 Nitro $\mathrm{OPE} \ldots \ldots \ldots \ldots \ldots \ldots \ldots \ldots \ldots$

D Conversion Factor between External Electric Field and Bias Voltage 147

E Mulliken Charge Distributions of Bistable [2/Rotaxane Molecular Switch Depend-

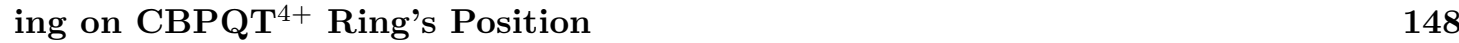

F Consideration of Metric Effect on the Bistable [2/Rotaxane Molecule during the $\begin{array}{ll}\text { Constant MD Simulations using Fixman's Theorem } & 156\end{array}$

G Time for Consumption of POPG $\quad 158$

H Bulk-phase Ozonolysis $r$

H.1 Methods . . . . . . . . . . . . . . . . . . . . . . . 159

H.1.1 Chemicals and Reagents . . . . . . . . . . . . . . . 159

H.1.2 Bulk-phase $\mathrm{O}_{3}$ Reaction . . . . . . . . . . . . . . . . . . 159

H.1.3 Fenton Reaction . . . . . . . . . . . . . . . . . . . . . . 159

H.2 Results and Discussion . . . . . . . . . . . . . . . . . . . 160

Bibliography $r \begin{aligned} & 165\end{aligned}$ 


\section{List of Figures}

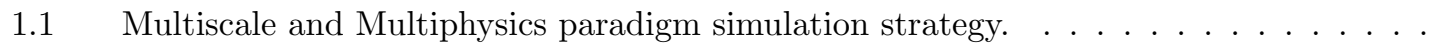

1.2 Multiscale and Multiphysics characteristics in nano electronic systems. At electron size scale, electrical conductivity depends on the quantum states of the molecules. At molecular size scale, classical interactions determine the molecular conformation, which affects its quantum state. At device size scale, hundreds of molecules are assembled to build a mesoscale junction and these are packaged into a device component. . . . . .

1.3 Multiscale and Multiphysics characteristics in bio systems. At electron size scale, chemical reactions, which are governed by huge QM effects (e.g., electron/proton transfer), regulate the molecular architecture of the protein for an effective functionality. At molecular scale, CM decribes the interactions, such as hydrophobic interaction, among the bio components of proteins, lipids, etc. Large scale motion of the membrane, such as endo- or exo-vesiculation, is in the regime of hydrodynamic descriptions, and this affects the dynamics of a cell.

2.1 (a) Energies of various conformations of AN-OPE relative to the AP conformation computed with a $1.2 \mathrm{~V} / \AA$ external field from FF calculations (black histograms) and NN model calculations (orange histograms). (b) Energies of various conformations of AN-OPE relative to the AP conformation computed with no external field from FF calculations (black histograms), NN model calculations (orange histograms), and QM

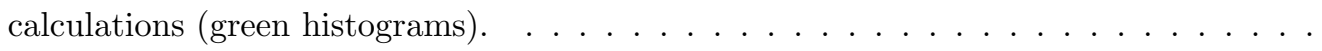

2.2 Torsional strain energy $E_{i}^{\text {torsion }}$ as a function of twisting angle $\chi$ from QM (PBE) on the isolated molecule. The energy at $\chi=4^{\circ}$ (corresponding to $\mathrm{P}$ ) is $0.01 \mathrm{kcal} / \mathrm{mol}$ higher than the ground state energy at $\chi=0^{\circ}$. The energy at $\chi=160^{\circ}$ (which corresponds to T) is $0.23 \mathrm{kcal} / \mathrm{mol}$ higher than the energy at $\chi=0^{\circ}$, leading the 0.22 $\mathrm{kcal} / \mathrm{mol}$ higher $E_{i}^{\text {torsion }}$ of T compared to $E_{i}^{\text {torsion }}$ of P. . . . . . . . . . . 
2.3 (a) Optimized geometry for the low field structure (P) of AN-OPE SAM. Here [0001] is the surface normal and the views are along $z$-axis (upper middle), $y$-axis (lower middle), and $x$-axis (lower right). The left picture is a perspective along the axis of one plane of molecules. The hydrogen bonding network is aligned along the [11 $\overline{2} 0]$ direction. (b) Optimized geometry for the high field structure (T) of AN-OPE SAM. Here [0001] is the surface normal and the views are along $z$-axis (upper middle), $y$-axis (lower middle), and $x$-axis (lower right). The left picture is a perspective along the axis of one plane of molecules. The hydrogen bonding network is aligned along the [1010] direction. . . . . . . . . . . . . . . . . . . . . . .

2.4 Density of state (DOS) and transmission function $T(E)$ of $\mathrm{P}$ structure (a) and $\mathrm{T}$ structure (b). The top electrode is located $12 \AA$ above from the bottom electrode. . .

2.5 (a) Current through $\mathrm{P}$ conformation, $I_{P}$ and current through $\mathrm{T}$ conformation, $I_{T}$ versus the bias voltage $V$ determined from the NEGF calculations using the DOS and $\mathrm{T}(\mathrm{E})$ of Figure 2.4. (b) Conductivity of $\mathrm{P}$ conformation, $\sigma_{P}$ and conductivity of $\mathrm{T}$ conformation, $\sigma_{T}$ versus the bias voltage $V$ determined. The top electrode is located $12 \AA$ above from the bottom electrode. $\sigma_{P}$ is $\sim 10$ times larger than $\sigma_{T} . \ldots \ldots \ldots$

2.6 Density of state (DOS) and transmission function $T(E)$ of $\mathrm{P}$ structure (a) and $\mathrm{T}$ structure (b). The top electrode is located $20 \AA$ above from the bottom electrode. . .

2.7 (a) Current through $\mathrm{P}$ conformation, $I_{P}$ and current through $\mathrm{T}$ conformation, $I_{T}$ versus the bias voltage $V$ determined from the NEGF calculations using the DOS and $\mathrm{T}(\mathrm{E})$ of Figure 2.6. (b) Conductivity of $\mathrm{P}$ conformation, $\sigma_{P}$ and conductivity of $\mathrm{T}$ conformation, $\sigma_{T}$ versus the bias voltage $V$ determined. The top electrode is located $20 \AA$ above from the bottom electrode. $\sigma_{P}$ is $\sim 163$ times larger than $\sigma_{T} . \quad \ldots \ldots$. .

2.8 Conductivities depending on the twisting angle are extracted from the previous study [36], which are the results from NEGF calculations. (a) Semi-log plots of $I-V$ curves shows that the slope decrease as the twist angle approaches to $90^{\circ}$, which means that the tunneling barrier from the bottom electrode to top electrode, $\Phi_{B}$ increases as the the $\pi-\pi$ orbital overlap decreases. (b) Since the conductivity is dominated by the $\pi-\pi$ orbital overlap, we assumed that the conductivity between two phenyl rings, $\sigma_{i}=1 / R_{i}$ is proportional to $\cos ^{4}\left(\chi_{i}\right)$. From simple calculation leads the total conductivity tot to be proportional to the $\cos ^{4}\left(\chi_{1}\right) \cos ^{4}\left(\chi_{2}\right) /\left(\cos ^{4}\left(\chi_{1}\right)+\cos ^{4}\left(\chi_{i}\right)\right)$, which shows good agreement with the NEGF results. 
2.9 (a) Snapshots during P-to-T transition from MC simulation with $1.2 \mathrm{~V} / \AA$ A external field. The first nucleation is occurred at 55, $184 \mathrm{MCS}$, then, the [1120] line propagates until 55, 214 MCS. More nucleation and propagation along [11 $\overline{2} 0]$ are taken place, and finally, the full system is transformed by 1,008, 706 MCS. We note that increased bias voltage yields a faster P-to-T transition. (b) Time dependence of $\mathrm{P}$ and $\mathrm{T}$ populations and electrical conductivity through the SAM during P-to-T transition. (c) Snapshots during T-to-P transition from MC simulation without an external field. The first nucleation is occurred at 486,389 MCS, then, the [11̄0] line propagates until 486, $410 \mathrm{MCS}$. More nucleation and propagation along [1120] are taken place, and finally, the full system is transformed by 2,377,335 MCS. (d) Time dependence of $\mathrm{P}$ and $\mathrm{T}$ populations and electrical conductivity through the SAM during T-to-P transition. . . . . . . . . .

2.10 (a) P-to-T transition; (b) T-to-P transition; Top panels show the temperature dependence of the propagation rate along $[11 \overline{2} 0], k_{[11 \overline{2} 0]}$. Middle panels show the probability, $P(t)$ of exhibiting no nucleation by time, $t$ for the case when the nucleation is initiated next to another $[11 \overline{2} 0]$ line. The dotted lines is an exponential fit of $P(t)$. This leads to a nucleation rate of $\tau=5,583$ MCS for the P-to-T transition and $\tau=72,926$ MCS for the T-to-P transition. Bottom panels show the probability, $P(t)$ of exhibiting no nucleation by time, $t$ for the case when the nucleation is initiated in the absence of next $[11 \overline{2} 0]$ line. The exponential fit leads to a nucleation rate of $\tau_{0}=274,193 \mathrm{MCS}$ for the P-to-T transition and $\tau_{0}=357,135$ MCS for the T-to-P transition. . . . . . .

2.11 (a) Current-voltage $(I-V)$ curves calculated at $300 \mathrm{~K}$ from MC simulations combined with the $I-V$ results of the Green's function calculations. Results for three sweeping rates are shown: $\mathrm{S}$ (blue line): $1 \times 10^{-8} \mathrm{~V} / \mathrm{MCS}, \mathrm{M}$ (red line): $4 \times 10^{-8} \mathrm{~V} / \mathrm{MCS}$, and F (black line): $2 \times 10^{-7} \mathrm{~V} / \mathrm{MCS}$. Inset is the experimental $I-V$ curves from the reference [9] with 3 different sweeping rates: $\mathrm{S}$ (blue line) : $21 \mathrm{mV} / \mathrm{sec}, \mathrm{M}$ (red line) : $83 \mathrm{mV} / \mathrm{sec}$, and $\mathrm{F}$ (black line) : $415 \mathrm{mV} / \mathrm{sec}$. (b) The sweep rate dependence of the integral of the current from the peak to the valley during NDR, $Q_{F}$ from current simulation (left panel) and experiment $[9]$ (right panel). . . . . . . . . . . . . .

3.1 Structural formula of the two co-conformations of a bistable [2]rotaxane fragment used

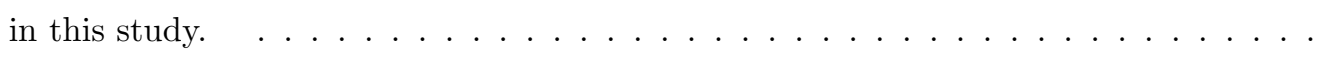

3.2 (a) Backbone of the rotaxane molecule simulated in this study. (b) $\mathrm{CBPQT}^{4+}$ ring positions along the backbone (unit: $\AA$ ) . . . . . . . . . . . . . . . . . 
3.3 Charges for the initial nine structures obtained from QM with Mulliken analysis. In addition, we included two more structures beyond each station of the TTF and the DNP, using charges identical to those for the equilibrium CBPQT ${ }^{4+} @ \mathrm{TTF}$ and $\mathrm{CBPQT}^{4+} @ \mathrm{DNP}$ cases, respectively. Ten more structures were generated on the basis of these eleven structures. The position of the $\mathrm{CBPQT}^{4+}$ ring for each additional structure was obtained using the arithmetic average of the two adjacent cases from the eleven structures. The charges were also averaged.

3.4 Probability density function of kinetic energy $\mathrm{KE}\left(=m v^{2} / 2\right)$ is from the $\mathrm{MD}$ simulation of the $\mathrm{CBPQT}^{4+}$ ring@TTF (blue line) at $300 \mathrm{~K}$. Here the time step was $0.01 \mathrm{fs}$ and the total simulation time was $3 \mathrm{~ns}$ after $500 \mathrm{ps}$ of equilibration. The black dashed line compares with the Maxwell-Boltzmann distribution of the energy, $2\left(\mathrm{KE} / \pi\left(k_{B} T\right)^{3}\right)^{1 / 2} \exp \left(-\mathrm{KE} / k_{B} T\right)$, for $T=300 \mathrm{~K} . \ldots \ldots \ldots$

3.5 Probability density function of $\mathrm{KE}_{x}\left(=m v_{x}^{2} / 2\right.$; blue line $), \mathrm{KE}_{y}\left(=m v_{y}^{2} / 2\right.$; green line), and $\mathrm{KE}_{z}\left(=m v_{z}^{2} / 2\right.$; red line $)$ are cmputed from the MD simulation of the CBPQT ${ }^{4+}$ ring@TTF (blue line), which are identical to each other. These are compared with the Maxwell-Boltzmann distribution of $\mathrm{KE}_{i}, \sqrt{1 /\left(\pi \mathrm{KE}_{i} k_{B} T\right)} \exp \left(-\mathrm{KE}_{i} / k_{B} T\right)$ at $300 \mathrm{~K}$. $(i \in\{x, y, z\}$; black dashed line $) \ldots \ldots \ldots \ldots \ldots$

3.6 Change of mean force as a function of simulation time. In each case this follows 500 ps of equilibration time. This plot shows two representative cases: the CBPQT ${ }^{4+}$

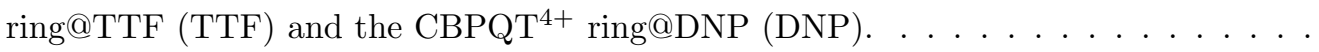

3.7 Change of potential of mean force as a function of ring position along the backbone. The blue curve allows the charge to change adiabatically as the ring moves along the dumbbell, which is the reliable result. The other two curves show the error obtained when the charges are fixed: the green curve uses fixed charges from the ring@TTF; the red curve uses a fixed charge from the ring@DNP. . . . . . . . . . . . . .

3.8 (a) Change of the mean force as a function of ring position along the backbone. (b) Change of the potential of mean force as a function of ring position along the backbone. The green vertical lines denote the ring@TTF (8.90 $\AA)$ and ring@DNP $(47.70 \AA)$. . .

3.9 Change of Coulombic interaction energy as a function of the ring position: (a) neutral state; (b) oxidation state +1 ; (c) oxidation state +2 .

3.10 Change of van der Waals interaction energy as a function of the ring position: (a) neutral state; (b) oxidation state +1 ; (c) oxidation state +2 . . . . . . . . . . . .

3.11 Variations in the total charge on the ring as a function of the ring position for the neutral case. 
4.1 Atomistic structure for the primitive periodic cell with composition of $\mathrm{Na}_{8} \mathrm{Al}_{8} \mathrm{Si}_{248} \mathrm{O}_{512}$. This shows green balls are aluminum and magenta balls are sodium. (a) Minimized aluminosilicate zeolite BEA structures without $\mathrm{H}_{2} \mathrm{O}$, (b) snapshot of zeolite BEA structures after the GCMC simulation for $298.15 \mathrm{~K}$ and $101.3250 \mathrm{kPa}$, and (c) snapshot of zeolite BEA structures after $4 \mathrm{~ns}$ NPT MD simulation at $298.15 \mathrm{~K}$ and $101.3250 \mathrm{kPa}$.

4.2 Dashed line is the least-squares fitted to $f(x)=a \tanh (b x)$, where $a=46.19$ molecules/ unit cell and $b=0.753(1 / \mathrm{kPa})$. We obtain a vapor pressure of $3.578 \mathrm{kPa}$ for the point at which the water uptake reaches to $99 \%$ of the maximum loading uptake. . . . . .

4.3 Predicted temperature-dependence of water uptake at $101.3250 \mathrm{kPa}$ when the zeolite $\mathrm{BEA}$ has no aluminum doping (red curve) and $\mathrm{Si} / \mathrm{Al}=30$ (blue curve). The presence of aluminum-doped sites as well as sodium ions enhance the water uptake amount especially in the high temperature. . . . . . . . . . . . . . . . . .

4.4 (a) Probability of finding additional water molecules at a specific distance from each water molecule. This is obtained as the product of pair correlation functions of water oxygens, $g_{O \text { (water })-O(\text { water })}$ with water density $\rho$ and $4 \pi r^{2}$. (b) Water coordination number $(\mathrm{CN})$ of water molecule as a function of pressure. The $\mathrm{CN}$ of water molecule is obtained from the integration of curve a up to first minimum, which is at $3.7 \AA$. The saturated value of $\mathrm{CN}$ of the absorbed phase $(\sim 3.8)$ is smaller than the $\mathrm{CN}$ of the bulk

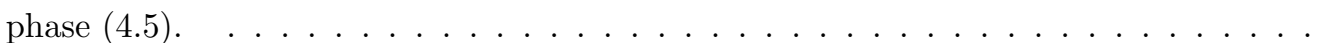

4.5 (a) Probability of finding sodium ions at a specific distance from each water molecule. This is obtained as the product of pair correlation functions of sodium atoms and water oxygen, $g_{N a-O(w a t e r)}$ with water density $\rho$ and $4 \pi r^{2}$. (b) Water coordination number $(\mathrm{CN})$ of sodium ions as a function of pressure. The $\mathrm{CN}$ of water molecule is obtained from the integration of curve a up to first minimum, which is at $3.5 \AA$. The saturated value of $\mathrm{CN}$ of the absorbed phase $(\sim 5.0)$ is same to the $\mathrm{CN}$ of the bulk phase. . . .

4.6 Log-log plot of mean square displacements (MSD) vs time at 298.15 K and 101.3250 $\mathrm{kPa}$ based on a trajectory of $12 \mathrm{~ns}$. Initially the MSD shows MSD $\propto t^{1 / 2}$ up to $t=\sim 20$ ps, but the times $>\sim 2$ ns, we see normal 3D Fickian behavior $(\mathrm{MSD} \propto t)$. The Fickian regime leads to a diffusion coefficient of $D=1.233 \times 10^{-7} \mathrm{~cm}^{2} / \mathrm{s}$. . . . . . . . . 
xvii

4.7 Dependence of diffusion coefficient of sodium ion on water coordination number $(\mathrm{CN})$ of sodium ion. The diffusion coefficients were calculated from three partitioned trajectories of 12 ns NPT simulations (each partition has 4 ns length), and the error bars were evaluated from the diffusion coefficients obtained from the three partitioned trajectories. This represents pressures from 0.1013 to $101.3250 \mathrm{kPa}$ at $298.15 \mathrm{~K}$. At a pressure of $\sim 2.0 \mathrm{kPa}$, we find an abrupt change in the water $\mathrm{CN}$. When the sodium ion is solvated by $>4.5$ water molecules, its diffusion coefficient increases abruptly by a factor of 3.5. The dashed line is to guide the eye. . . . . . . . . . . . . .

4.8 Time dependence of the distances of one sodium ion to all 8 aluminum atoms of the zeolite membrane. The distance to each aluminum atom is shown with a different color. (a) Vapor pressure is $101.3250 \mathrm{kPa}$ (good solvation regime) at $298.15 \mathrm{~K}$. This shows that it took $2 \mathrm{~ns}$ for the $\mathrm{Na}$ to hop from a position $4.8 \AA$ from the purple $\mathrm{Al}$ to a position $7.5 \AA$ from the black Al. Here a distance of $4.8 \AA$ indicates one water shell between the $\mathrm{Na}$ and the $\mathrm{Al}$, while $7.5 \AA$ indicates separate water shells around each; (b) vapor pressure is $0.1013 \mathrm{kPa}$ (poor solvation regime) at $298.15 \mathrm{~K}$. In this case the $\mathrm{Na}$ remains at $4 \AA$ from the $\mathrm{Al}$ which indicates that the $\mathrm{Na}$ remains coordinated to the

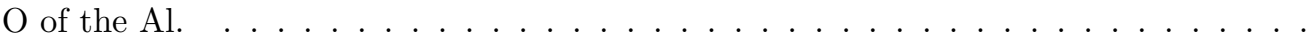

4.9 Dependence of the sodium diffusion coefficient on the temperature for a vapor pressure of $101.3250 \mathrm{kPa}$. The diffusion coefficients were calculated from three partitioned trajectories of 12 ns NPT simulations (each partition has 4 ns length), and the error bars were evaluated from the diffusion coefficients obtained from the three partitioned trajectories. Up to $373.15 \mathrm{~K}$, the diffusion coefficient increases with increasing temperature, whereas it decreases beyond $373.15 \mathrm{~K}$ due to the depletion of the water solvation.

4.10 Arrhenius plots of diffusion coefficient from each solvation regime: the red squares are for the poor solvation regime and the blue diamond is for the good solvation regime. The solid black line is a linear fitting of each regime. This leads to $D_{300 K}=3.762 \times 10^{-8}$ $\mathrm{cm}^{2} / \mathrm{s}$ and $E_{a c t}=3.810 \mathrm{kcal} / \mathrm{mol}$ for the poor solvation regime and $D_{300 K}=1.512 \times$ $10^{-7} \mathrm{~cm}^{2} / \mathrm{s}$ and $E_{a c t}=3.540 \mathrm{kcal} / \mathrm{mol}$ for the good solvation regime, and the equation of each fitted line and R2 value are inset in the figure. 
xviii

5.1 Examples of the ion mobility spectra taken in this study. Shown are two spectra taken in 730 Torr $\mathrm{N}_{2}$. The electric field strength and the temperature of the drift tube were $292 \mathrm{~V} / \mathrm{cm}$ and $473 \mathrm{~K}$, respectively. The dash curve is a spectrum taken with pure solvent being introduced to the electrospray needle while the solid curve is a spectrum of solvent and $300 \mu \mathrm{M} N, N$-dimethylammoniumethanol. The two spectra were smoothed (10 point adjacent averaging) and shifted in intensity by an additive constant to avoid overlap. The $N, N$-dimethylammoniumethanol feature is indicated in the figure. The unlabeled features correspond to ionized solvent (water, methanol, acetic acid) and atmospheric constituents ionized through proton transfer (due to the open nature of the ESI-IMS instrument). . . . . . . . . . . . . .

5.2 Plot of $K_{0}^{-1}$ for $3^{\circ}$ and $4^{\circ}$ ammonium cations drifting in $\mathrm{N}_{2}$ versus ion mass. Experimentally determined data for $3^{\circ}$ ammonium and $4^{\circ}$ ammonium cations are shown as asterisks and solid squares, respectively. The solid line is the fit of the $12-4$ potential model to the ammonium cation data set. DFT optimized structure of each numerically or alphabetically labeled ion is shown above. Optimized geometries are obtained at B3LYP/6-31G** level. The hydrogen bonds are indicated with dashed lines. . . . . .

5.3 (a) Plot of experimentally determined collision cross-sections $\left(\Omega_{D}\right)$ of abiotic amino acid cations [14] and carboxylic acid anions [15] in $\mathrm{N}_{2}$ versus theoretically determined $\Omega_{D}$ using the modified TJ method for $\mathrm{N}_{2}$ drift gas. Abiotic amino acid cation data are shown as solid squares and carboxylic acid anion data are shown as empty circles. The solid line is $y=x$. (b) Plot of experimentally determined collision cross-sections $\left(\Omega_{D}\right)$ of $3^{\circ}$ and $4^{\circ}$ ammonium cations in $\mathrm{N}_{2}$ versus theoretically determined $\Omega_{D}$ using the modified TJ method for $\mathrm{N}_{2}$ drift gas. $3^{\circ}$ ammonium cation data are shown as asterisks and $4^{\circ}$ ammonium cation data are shown as solid squares. Each ion is labeled with the appropriate identifying number and alphabet shown in Figure 5.1. The solid line

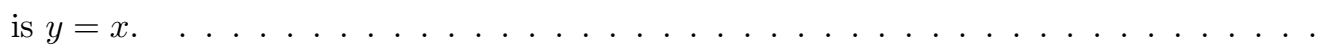

$5.4 \quad$ Plots of theoretically determined collision cross-sections $\left(\Omega_{D}\right)$ (a) with potential from van der Waals and ion-induced dipole $(V D W+I I D)$ interactions over the theoretical $\Omega_{D}$ with original pairwise potential, van der Waals + ion-induced dipole + ionquadrupole $(V D W+I I D+I Q)$ interactions, and (b) with potential from van der Waals potential $(V D W)$ over the theoretical $\Omega_{D}$ with potential from van der Waals and ion-induced dipole $(V D W+I I D)$ interactions of $3^{\circ}$ and $4^{\circ}$ ammonium cations, abiotic amino acid cations, and carboxylic acid anions in $\mathrm{N}_{2}$ versus ion mass. The ammonium cation data, the abiotic amino acid cation, and carboxylic acid anion data are shown as solid squares, empty circles, and asterisks, respectively. . . . . . . . 
5.5 Plots of theoretically determined collision cross-sections $\left(\Omega_{D}\right)$ of $3^{\circ}$ and $4^{\circ}$ ammonium cations, abiotic amino acid cations, and carboxylic acid anions in $\mathrm{N}_{2}$ versus ion mass. The calculated $\Omega_{D}$ of the molecular ions only with van der Waals $(V D W)$ interaction with $\mathrm{N}_{2}$ are shown as empty squares (left $y$-axis). The calculated $\Omega_{D}$ of the molecular ions with original pairwise potential, van der Waals + ion-induced dipole + ion-quadrupole $(V D W+I I D+I Q)$ interactions, with $\mathrm{N}_{2}$ are shown as solid circles

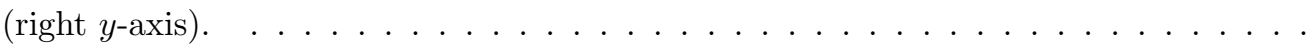

5.6 Plot of the total shape asymmetry (AS) of the ammonium cations versus ion mass. The ammonium cations with no oxygen atom are shown as solid squares. The ions containing one oxygen atom and two oxygen atoms are shown as empty triangles and empty circles, respectively. The DFT optimized structure of each numerically or alphabetically labeled ion is shown in Figure $5.2 . \ldots \ldots \ldots$. . . . . . . . . .

6.1 (a) Plot of drift time of saturated phosphatidylcholine (PC) cations in traveling wave ion mobility spectrometer versus ion mass. Experimentally determined data for symmetric PC and asymmetric PC cations are shown as solid squares and empty circles, respectively. The black dash and red solid lines are the linear fit to the symmetric PC cation data set and to both symmetric and asymmetric PC cation data set, respectively. (b) Plot of drift time of PC cations spanning mass range 700-800 Da in traveling wave ion mobility spectrometer versus ion mass. Experimentally determined data for saturated PC and unsaturated PC cations are shown as solid squares and empty circles, respectively. The black dash and red solid lines are the linear fit to the saturated PC cation data set and to both saturated and unsaturated PC cation data set, respectively. (c) Plot of drift time of protonated and sodiated PC cations in traveling wave ion mobility spectrometer versus ion mass. Experimentally determined data for protonated PC and sodiated PC cations are shown as solid squares and empty circles, respectively. The black dash and red solid lines are the linear fit to the protonated PC cation data set and to both protonated and sodiated PC cation data set, respectively.

6.2 (a) Plot of corrected empiric cross-sections versus effective drift times for 14 peptides and 4 phosphatidylcholines (PC). For each peptide and PC the singly charged cation is used. Linear trend and power trend lines are shown as solid and dash lines, respectively. (b) A plot of the estimated cross-sections versus the ion mass for PC cations investigated in this study. The estimated collision cross-sections from linear trend and power trend are shown as solid squares and empty circles, respectively. . . . . . . . . . . . . . 10 
6.3 (a) Time profile of the distance between the carbon atoms at the end of each acyl chain of 18:0-18:0 phophatidylcholine during 200 ps of the molecular dynamics simulation. The fluctuation is ranging from $\sim 5 \AA$ to $\sim 25 \AA$ with the time period of $5-20$ ps. Approximately 17 times of fluctuation is observed from this trajectory. (b) Plot of experimentally determined collision cross-sections $\left(\Omega_{D}\right)$ of phosphatidylcholine (PC) cations in $\mathrm{N}_{2}$ against theoretically determined $\Omega_{D}$ using the modified TJ method for $\mathrm{N}_{2}$ drift gas. The theoretical $\Omega_{D}$ is obtained by averaging $\Omega_{D}$ for 200 structures from MD simulations. The solid line is $y=x$. (c) Plot of theoretical $\Omega_{D}$ in He over theoretical $\Omega_{D}$ in $\mathrm{N}_{2}$ versus mass of PC cations. . . . . . . . . . . . . . . . . . . 107

6.4 (a) Plots of theoretically determined collision cross-sections $\left(\Omega_{D}\right)$ and surface areas of phosphatidylcholine (PC) cations in $\mathrm{N}_{2}$ versus ion mass. The calculated average $\Omega_{D}$ of the 200 ion conformations are shown as solid squares (left $y$-axis). The calculated surface areas of $\mathrm{PC}$ cations in $\mathrm{N}_{2}$ at $300 \mathrm{~K}$ are shown as empty circles (right $y$-axis). (b) Plots of experimentally evaluated $\Omega_{D}$ and surface areas of phosphatidylcholine (PC) cations in $\mathrm{N}_{2}$ versus ion mass. The $\Omega_{D}$ of PC cations are shown as solid squares (left $y$-axis). The calculated surface areas of PC cations in $\mathrm{N}_{2}$ at $300 \mathrm{~K}$ and $400 \mathrm{~K}$ are shown as empty circles and empty triangle, respectively (right $y$-axis). . . . . . . . . 110

6.5 (a) MD simulated structures of saturated phosphatidylcholine cations at minimum energy state $\left(E_{0}\right)$ are shown. The structures of the closest $\Omega_{D}$ to the experimental values are also shown along with corresponding relative energy values $\left(E^{*}\right)$. (b) Plot of experimentally determined collision cross-sections $\left(\Omega_{D}\right)$ of phosphatidylcholine (PC) cations in $\mathrm{N}_{2}$ against theoretically determined $\Omega_{D}$ at $E_{0}$ and $E^{*}$ using the modified TJ

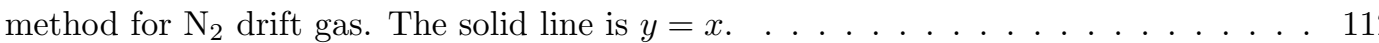

6.6 MD simulated structures of unsaturated phosphatidylcholine cations at minimum energy state $\left(E_{0}\right)$ are shown. The structures of the closest $\Omega_{D}$ to the experimental values are also shown along with corresponding relative energy values $\left(E^{*}\right)$. Unsaturated acyl chain is colored in yellow. 
7.1 Illustration of FIDI-MS methodology for studies of interfacial reactions. (a) A quiescent hanging droplet of analyte-containing solution is formed on the end of a capillary. All electrical components remain at ground as the droplet is formed and reacts in a fieldfree environment. (b) The droplet is exposed to gas-phase reactants for a variable period of time to allow for heterogeneous reactions between gas-phase and solutionphase species. (c) After a reaciton period, a pulsed electric field stretches the neutral droplet until it emits stream of positively and negatively charged submicron droplets in opposite directions. Ionized reactants and products from heterogeneous reactions enter the capillary inlet of the mass analyzer. Either positive or negative ions can be sampled. . . . . . . . . . . . . . . . . . . . 122

7.2 Structures of POPG, POG, and SP-B ${ }_{1-25}$ investigated in this study. . . . . . . . . . 123

7.3 (a) Heterogeneous reaction of POPG with $\mathrm{O}_{3}$ as a function of time. In the absence of ozone, the negative ion FIDI-MS spectrum of POPG is dominated by the singly deprotonated POPG peak at m/z 748. POPG is depleted after $15 \mathrm{~s}$ of the exposure and oxidation products are dominated by deprotonated hydroxyhydroperoxide (HHP) at $\mathrm{m} / \mathrm{z}$ 672. The aldehyde, carboxylic acid, and methoxyhydroperoxide products are observed at $\mathrm{m} / \mathrm{z} 638, \mathrm{~m} / \mathrm{z} 654$, and $\mathrm{m} / \mathrm{z} 686$, respectively. The secondary ozonide (SOZ) and sodiated alcohol products show up in the spectra at $\mathrm{m} / \mathrm{z} 796$ and $\mathrm{m} / \mathrm{z}$ 662 , respectively. (b) Summary of heterogeneous oxidation of POPG with $\mathrm{O}_{3}$ at the air-liquid interface. $\mathrm{R}^{\prime}$ is $\mathrm{H}$ for water and $\mathrm{CH}_{3}$ for methanol. . . . . . . . . . . . . .

7.4 (a) Air-liquid interfacial oxidation of $\mathrm{SP}_{-1-25}$ by $\mathrm{O}_{3}$ as a function of time. In the absence of ozone, the positive ion FIDI-MS spectrum of SP-B ${ }_{1-25}$ is dominated by the doubly protonated $\mathrm{SP}_{-} \mathrm{B}_{1-25}$ peak at $\mathrm{m} / \mathrm{z} 1465$. The products at $\mathrm{m} / \mathrm{z} 1481$ and $\mathrm{m} / \mathrm{z}$ 1489 correspond to doubly protonated $\mathrm{SP}-\mathrm{B}_{1-25}$ with two oxygen atoms and with three oxygen atoms, respectively, appear after the droplet is exposed to $\mathrm{O}_{3}$ for $5 \mathrm{~s}$. The triply oxygenated product at $\mathrm{m} / \mathrm{z} 1489$ dominates the FIDI-MS spectrum after exposing the droplet to $\mathrm{O}_{3}$ for $10 \mathrm{~s}$. No further oxidation of the peptide is observed up to $30 \mathrm{~s}$ of exposure. (b) FIDI-MS ${ }^{2}$ of doubly charged triply oxygenated $\mathrm{SP}_{-} \mathrm{B}_{1-25}$ product from heterogeneous oxidation yields an exclusive fragment at m/z 1457 resulting from the elimination of hydrosulfinylmethane $\left(\mathrm{CH}_{4} \mathrm{SO}\right)$. (c) The oxidation mechanisms of Trp by ozonolysis and hydrolysis with hydrolysis mechanism of Met in peptide. (d) Airliquid interfacial oxidation of $\mathrm{SP}_{-} \mathrm{B}_{1-25}$ by $\mathrm{O}_{3}$ in the POG monolayer as a function of time. Doubly protonated SP-B $1-25$ products with two oxygen atoms and with three oxygen atoms appear after the droplet is exposed to $\mathrm{O}_{3}$ for $10 \mathrm{~s}$. The triply oxygenated product dominates the FIDI-MS spectrum after exposing the droplet to $\mathrm{O}_{3}$ for $15 \mathrm{~s}$ and no further oxidation of the peptide is observed up to $30 \mathrm{~s}$ of exposure. 
7.5 Atomic density profiles of POPG monolayer systems (solid lines) and POG monolayer systems (dotted lines) as a function of $\Delta z$, where the air/liquid interface is 0 , and proceeding left to right from the water to the lipid layer. The lipid surface densities are (a) $55 \AA^{2} /$ lipid, (b) $60 \AA^{2} /$ lipid, (c) $65 \AA^{2} /$ lipid, and (d) $70 \AA^{2} /$ lipid. Blue lines denote the density profiles of oxygen atoms of water molecules, black lines denote that of saturated carbons of lipid acyl chains, and red lines denote that of unsaturated carbons of lipid acyl chains

7.6 (a) Final snapshot after 2.0 ns of MD simulation of SP-B $\mathrm{B}_{1-25}$ in a POG monolayer at 60 $\AA^{2}$ /lipid. The peptide is shown in rainbow color (C-terminal: red, N-terminal: blue). Lipids, water molecules, and chloride are shown in purple, cyan, and red, respectively. (b) AA hydrophobicities [46] (top) and $\Delta z$ of $\mathrm{C}_{\alpha}$ of each residue averaged during the last $0.5 \mathrm{~ns}$ of a $2.0 \mathrm{~ns}$ duration MD simulation (bottom) are plotted as a function of amino acid residue number. The air/water interface is located near $\Delta z=0$. (c) Atomic density profiles of SP-B $\mathrm{B}_{1-25}$ in POG monolayer at $60 \AA^{2} /$ lipid as a function of $\Delta z$ during the last $0.5 \mathrm{~ns}$ of the $2.0 \mathrm{~ns}$ MD simulation. Blue dash line denotes the density profiles of oxygen atoms of water molecules. Black and red dash lines denote those of separately summed saturated and unsaturated carbons of lipid acyl chains, respectively. Wine, magenta, cyan, and olive solid lines denote the 100 times scaled density profiles of the $\mathrm{C}_{\alpha}$ carbon of $\mathrm{Cys}_{8}$, Trp 9 , $\mathrm{Cys}_{11}$, Met 21 residues, respectively.

7.7 (a) The $x y$-projected density profiles of saturated carbon atoms of lipid acyl chains from MD simulations is shown with colors and the averaged positions of $\mathrm{C}_{\alpha}$ carbons of SP-B $\mathrm{B}_{1-25}$ in the POG monolayer is shown with a black line (each residue is shown with cross). (b) Top view of final snapshot after 2.0 ns of MD simulation of SP$\mathrm{B}_{1-25}$ in a POG monolayer at $60 \AA^{2} /$ lipid. The peptide is shown in rainbow color (C-terminal: red, N-terminal: blue). Lipids and water molecules are shown in gray and cyan, respectively. Black spheres denote unsaturated carbon atoms of lipid acyl chains, and orange spheres denote hydroxyl oxygen atoms. . . . . . . . . . . . . . 131 
7.8 (a) Final snapshot after $2.0 \mathrm{~ns}$ MD simulation of SP-B ${ }_{1-25}$ in POPG monolayer at 60 $\AA^{2} /$ lipid is shown at top panel. The peptide is shown in rainbow color (C-terminal: red, N-terminal: blue), lipids in gray, water molecules in cyan, and chloride ions in red. Lower left and right show that Arg residues and Lys residues (displayed with sticks) are interacting with phosphate groups of lipids, respectively. (Phosphorous atoms are in magenta.) (b) AA index for hydrophobicity scale [46] (top) and $\Delta z$ of $\mathrm{C} \alpha$ of each residue averaged during the last $0.5 \mathrm{~ns}$ trajectory of $2.0 \mathrm{~ns}$ duration MD simulations (bottom) are plotted as a function of amino acid residue number. The air/water interface is located near $\Delta z=0$. (c) Atomic density profiles of $\mathrm{SP}_{-} \mathrm{B}_{1-25}$ in POG monolayer at $60 \AA^{2} /$ lipid as a function of $\Delta z$ during the last $0.5 \mathrm{~ns}$ of the $2.0 \mathrm{~ns}$ MD simulation. Blue dash line denotes the density profiles of oxygen atoms of water molecules. Black and red dash lines denote those of saturated and unsaturated carbons of lipid acyl chains, respectively. Wine, magenta, cyan, and olive solid lines denote the 100 times scaled density profiles of the $\mathrm{C}_{\alpha}$ carbon of $\mathrm{Cys}_{8}, \operatorname{Trp}_{9}, \mathrm{Cys}_{11}$, and $\mathrm{Met}_{21}$

7.9 The $x y$-projected density profiles of saturated carbon atoms of lipid acyl chains is shown with colors and the averaged positions of $\mathrm{C}_{\alpha}$ carbons of $\mathrm{SP}-\mathrm{B}_{1-25}$ in the POPG monolayer is shown with a black line (each residue is shown with cross).

A.1 Total time taken for the complete P-to- $\mathrm{T}$ transition, $t_{\mathrm{P}-\mathrm{to}-\mathrm{T}}$ by varying the area of the periodic simulation cell as $10 \times 10=100,30 \times 30=900,40 \times 40=1600,50 \times 50=2500$, and $60 \times 60=3600$. Black line denotes the change of $t_{\mathrm{P} \text {-to- } \mathrm{T}}$ under the external field of $F=1.2 \mathrm{~V} / \AA$, red line denotes the change of $t_{\mathrm{P}-\text { to- } \mathrm{T}}$ scaled by 10 under the external field of $F=1.0 \mathrm{~V} / \AA$, and blue line denotes the change of $t_{\mathrm{P} \text {-to- } \mathrm{T}}$ scaled by 15 under the external field of $F=0.95 \mathrm{~V} / \AA$. The $t_{\mathrm{P} \text {-to-T }}$ value is converged at the area of $900-1600$ and 2500-3600 when $F=1.2 \mathrm{~V} / \AA$ and $1.0 \mathrm{~V} / \AA$, respectively. When $F=0.95 \mathrm{~V} / \AA$, the $t_{\mathrm{P}-\text { to- } \mathrm{T}}$ value is not converged until the area of 3600 . This infers that we need much larger simulation cell to get the right converged value of $t_{\mathrm{P}-t_{\mathrm{O}}-\mathrm{T}}$ as the $F$ approaches to the critical field, $F_{c}=0.56 \mathrm{~V} / \AA$. Otherwise, we will get the overestimated value of $t_{\mathrm{P} \text {-to-T }}$ near $F_{c}$.

B.1 (top panel) Electrical conductivity through the AN-OPE is estimated based on the twisting angles from MD simulations with $(10 \times 10)$ unit cell. (bottom panels) The final snapshot after each step is shown with the populations of $\chi_{1}$ and $\chi_{2}$, which are averaged during the last $0.5 \mathrm{~ns}$ dynamics. 
C.1 (a) Optimized geometry for the low field structure (P) of N-OPE SAM. Here [0001] is the surface normal and the views are along z-axis (upper middle), $y$-axis (lower middle), and $x$-axis (lower right). The left picture is a perspective along the axis of one plane of molecules. The weak hydrogen bonding network is aligned along the $[11 \overline{2} 0]$ direction. (b) Optimized geometry for the high field structure (T) of N-OPE SAM. Here [0001] is the surface normal and the views are along $z$-axis (upper middle), $y$-axis (lower middle), and $x$-axis (lower right). The left picture is a perspective along the axis of one plane of molecules. The weak hydrogen bonding network is aligned along the $[10 \overline{1} 0]$ direction. . . . . . . . . . . . . . . . . . . . .

C.2 (a) Energies of various conformations of N-OPE relative to the AP conformation computed with a $0.6 \mathrm{~V} / \AA$ external field from FF calculations (black histograms). (b) Energies of various conformations of N-OPE relative to the AP conformation computed with no external field from FF calculations (black histograms), and QM calculations (green histograms). . . . . . . . . . . . . . . . . . . . 146

E.1 Structures of a) backbone part and b) $\mathrm{CBPQT}^{4+}$ ring part with numbered atoms. The assigned numbers on the atoms are associated with the partial charge distribution data shown in Tables E.1, E.2, and E.3.

H.1 (a) Quadruply charged SP-B $\mathrm{B}_{1-25}$ and oxidized products from (b) the bulk-phase $\mathrm{O}_{3}$ reaction and (c) the Fenton reaction with intact SP-B $1-25$. (d) Triply charged SP$\mathrm{B}_{1-25}$ and oxidized products from (e) the bulk-phase $\mathrm{O}_{3}$ reaction and (f) the Fenton reaction are also shown. . . . . . . . . . . . . . . . . 160

H.2 (a) The ESI mass spectrum of the trypsin digests of SP-B $\mathrm{B}_{1-25}$ in positive mode. (b) The ESI mass spectrum of the trypsin digests of the oxidized SP-B ${ }_{1-25}$ from the bulkphase $\mathrm{O}_{3}$ reaction in positive mode. (c) The negative mode ESI mass spectrum of the trypsin digests of the oxidized $\mathrm{SP}-\mathrm{B}_{1-25}$ from the bulk-phase $\mathrm{O}_{3}$ reaction. . . . . . .

H.3 (a) The CID spectrum of cationic IQAMIPK $+3 \mathrm{O}$ at $\mathrm{m} / \mathrm{z} 817$ from the tryptic digest of the oxidized SP-B $1-25$. (b) The CID of cationic FPIPLPYCWLCR $+8 \mathrm{O}$ at $\mathrm{m} / \mathrm{z} 1636$ from the tryptic digest of the oxidized SP-B $1-25$. (c) The CID of anionic FPIPLPYCWLCR +80 at m/z 1634 from the tryptic digest of the oxidized SP-B $1-25.162$

H.4 ESI-MS ${ }^{2}$ of triply oxygenated products of quadruply protonated SP-B $1-25$ from (a) bulk-phase $\mathrm{O}_{3}$ application, and from (b) the Fenton reaction. $\mathrm{M}$ denotes a parent ion, which is triply oxygenated $\mathrm{SP}_{-1-25} \ldots \ldots \ldots \ldots \ldots$ 


\section{List of Tables}

2.1 AN-OPE Interaction Energies, $U_{i j}$, for Monte Carlo (MC) Calculations . . . . . . .

3.1 Free Energy Barriers, Rate Constants, and Relaxation Half-Lives from DNP toward TTF $(\mathrm{DNP} \rightarrow \mathrm{TTF})$ at $298 \mathrm{~K}$ (All Simulation Results from this Work) . . . . . . .

4.1 Vapor Pressures, Water Uptake/Cell, Water Coordination Numbers of the Water Molecule (up to $3.7 \AA$ Cutoff), Water Coordination Numbers of the Sodium Ion (up to $3.5 \AA$ Cutoff), and Diffusion Coefficients from NPT MD at Temperature of $298.15 \mathrm{~K}$. . . .

4.2 Temperature, Water Uptake/Cell, and Diffusion Coefficients at Constant Pressure Condition of $101.3250 \mathrm{kPa} \ldots \ldots \ldots \ldots$

5.1 Drift Times, Reduced Mobilities, and Collision Cross-Sections of Ammonium Cations

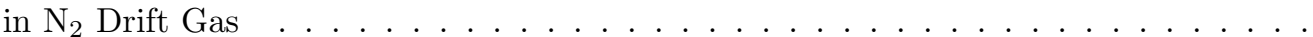

5.2 Critical Impact Parameter, $b^{*}$, Langevin Capture Cross-Section, $\Omega_{L}$, and Mean Relative Kinetic Energies, KE, during the Experiments with Experimentally Determined HardSphere Collision Radius, $R_{c}$ for Each Ammonium Cation . . . . . . . . . . . . . . . 86

5.3 Theoretically Determined Collision Cross-Sections of $3^{\circ}$ and $4^{\circ}$ Ammonium Cations . 87

6.1 Mass, Drift time, and Corrected Drift Time of Ion Molecule Used in This Study . . . 98

6.2 Optimized Partial Charge Distribution of Protonated Phosphate from the DFT Calculation of Protonated Dimethyl Phosphate (The Partial Charges with Atom Types for CHARMM Force Field are Tabulated.

6.3 Collision Cross-Sections of Phosphatidylcholine Cations in $\mathrm{N}_{2}$ Drift Gas Estimated and Evaluated using Empiric Calibration Method and Equations from Shavartsburg and Smith [27], respectively. Theoretically Determined Collision Cross-Sections in $\mathrm{N}_{2}$

6.4 Theoretically Determined Collision Cross-Sections $\left(\Omega_{D}\right)$ of Phosphatidylcholine Cations at Minimum Energy State $\left(E_{0}\right)$. The Differences of $\Omega_{D}\left(\Delta \Omega_{D}\right)$ and Potential Energy $(\Delta E)$ from the PC Structure at $E_{0}$ to Experimentally Determined $\Omega_{D} \ldots \ldots \ldots$ 
xxvi

E.1 Partial Charge Distribution of Neutral Rotaxane when the Center of Mass of the $\mathrm{CBPQT}^{4+}$ Ring Moves from $z=10.92 \AA$ (TTF side) to $z=44.83 \AA$ (DNP side) . . . 150

E.2 Partial Charge Distribution of +1 Oxidized Rotaxane when the Center of Mass of the $\mathrm{CBPQT}^{4+}$ Ring Moves from $z=10.92 \AA$ (TTF side) to $z=44.83 \AA$ (DNP side) . . . 152

E.3 Partial Charge Distribution of +2 Oxidized Rotaxane when the Center of Mass of the $\mathrm{CBPQT}^{4+}$ Ring Moves from $z=10.92 \AA$ (TTF side) to $z=44.83 \AA$ (DNP side) . . . 154 


\section{Chapter 1}

\section{Introduction}

Generally, a great number of chemical and physical problems in real life have multiscale and multiphysics features. Many observable physical quantities originate from multiple physical phenomena working cooperatively at different time- and length-scale. Interestingly, it is well known that different regimes are interpreted in terms of the different physics, which are inter-connected to each other through the "coarse-graining of the scale." Exemplifying, quantum mechanics (QM) governs the physics between nuclei and electrons. Classical mechanics (CM) governs the atomic or molecular dynamics on the complex energy surface, which is the outcome of the quantum mechanical interactions. The material flow is described by rheology, which coarse grains the particles into a continuum. Here, the governing parameters of such rheology are determined by the inter-molecular interactions.

Computational simulation methods, along with statistical mechanics, have been employed as a useful tool to expand our knowledge of microscale events and connect them into macroscopic observances. Thanks to the development of many simulation methods at various scales and the combination of these methods within a multiscale and multiphysics simulation paradigm (Figure 1.1), computational simulation is now regarded as an indispensable tool for the new advancement in science and engineering. In a good number of cases, computer simulations efficiently supplement experiments in interpreting observations, otherwise research would be too expensive or dangerous. On the contrary, in many others, computer simulations are the only possible choice to probe the complicated phenomena. In this context, the tremendous quest for ever-higher levels of detail and realism in such simulations, contribute to the inexorable demand for new theory, methods, and computational tools.

Currently, an enormous amount of research is focused on nano- and bio-systems. Research on nano-systems is most often centered on predicting nanoscaled transport properties such as electron, thermo, and mass transportations for the application of next-generation electronic devices or alternative energy materials. Research on bio-systems has significant potential in life science and medical applications. In particular, such nano- or bio-systems have a strong multiscale and multiphysical nature within them, intriguing the quest for the development of an elaborated methodology to un- 


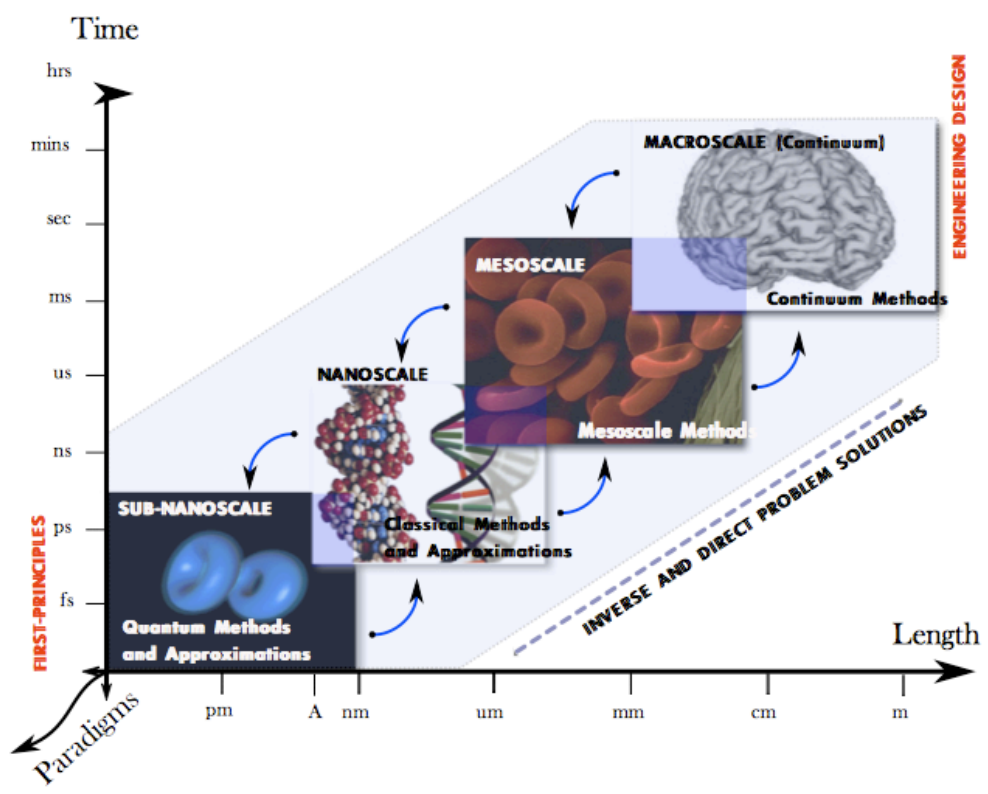

Figure 1.1: Multiscale and Multiphysics paradigm simulation strategy.

derstand the scale-dependent physics. Specifically, in nano-systems (Figure 1.2), electron transport through a molecule is strongly dependent on the energy eigen states of the molecule (QM regime), which are affected by the molecular/atomic conformations and motions (CM regime). When we design an engineering process for manufacturing electronic device such as ultimate complementary metal-oxide-semiconductor (CMOS) electronics using novel materials, continuum level theory and simulations are important. In bio-systems (Figure 1.3), lipid layers encapsulate cells and control fluidity and surface tensions, which are macroscopic/hydrodynamic regime quantities. These macroscopic changes are yielded by the inter-molecular interactions at the interface (CM region). In the lower scale, such molecular interactions are often tuned by chemical reactions such as a proton transfer, which has a strong QM effect.

Within contemporary computing power, direct use of first principle computational methods is limited by an order of $\sim 1000$ atoms and an order of pico to nano seconds. Thus, for managing many practical issues in using computational simulation methods which often involve a large lengthand long time-span, we need to overcome the limitations in simulation scales. In this thesis, several cases of nano- and bio-systems are presented; (1) we understand them by capturing fundamental nanoscale intrinsic and extensive properties, then, (2) we define the macroscopic behavior observed from experiments based on the microscopic understandings.

The first half (Chapters 2-4) discusses the multiscale and multiphysics simulation approaches for nano-systems.

In Chapter 2, we investigate a negative differential resistance (NDR) effect observed from a metal/molecular SAM/metal junction. Amino- and nitro-substituted oligo-phenylene ethynelene 


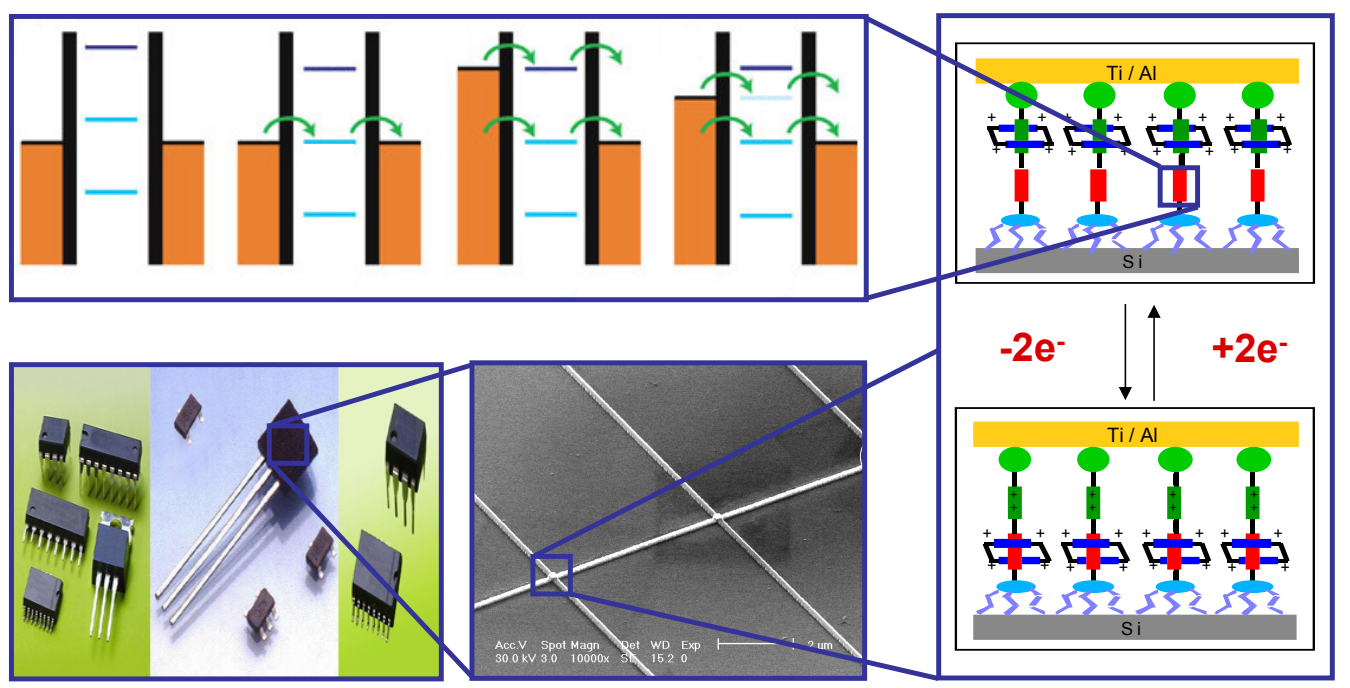

Figure 1.2: Multiscale and Multiphysics characteristics in nano electronic systems. At electron size scale, electrical conductivity depends on the quantum states of the molecules. At molecular size scale, classical interactions determine the molecular conformation, which affects its quantum state. At device size scale, hundreds of molecules are assembled to build a mesoscale junction and these are packaged into a device component.

(OPE) molecules in the self-assembled monolayer (SAM) have a large molecular dipole moment, which responds to the external electric field. Hence, applying the bias voltage induces the conformational changes of the individual moleculesand thereby yield a structural transition of the SAM. Such a conformational change is in the regime where the molecular dynamics (MD) simulation is useful. However, the kinetics during the structural relaxation of the SAM requires larger scale simulation methods. On the other hand, electrical conductivities on the total $I$ - $V$ curve are rooted in QM. In order to manage such a complicated problem, we design a nearest neighbor (NN) model with lattice Monte Carlo (MC) simulation, whose parameters are obtained by coarse-graining QM and MD simulation results. The electrical conductivities depending on the conformations are parameterized using non-equilibrium green functional theory combined with QM.

In Chapter 3, on/off kinetics of the molecular switch, bistable [2/rotaxane is discussed. The rotaxane has been intensively studied because of its interesting properties, which are attributed to the molecular recognition between charge donor and acceptor. The shuttling motion of electron accepting cyclobis-(paraquat- $p$-phenylene) $\left(\mathrm{CBPQT}^{4+}\right)$ between the charge donating stations such as tetrathiafulvalene (TTF) and 1,5-dioxynaphthalene (DNP) moieties, has brought many applications such as molecular switch in nanoelectronics and artificial muscle in nanomechanics. Therefore, comprehensive understanding of the free energy barrier required for this shuttling motion is essential. In addition, the fine control of the free energy barrier can open an application of the rotaxane system 


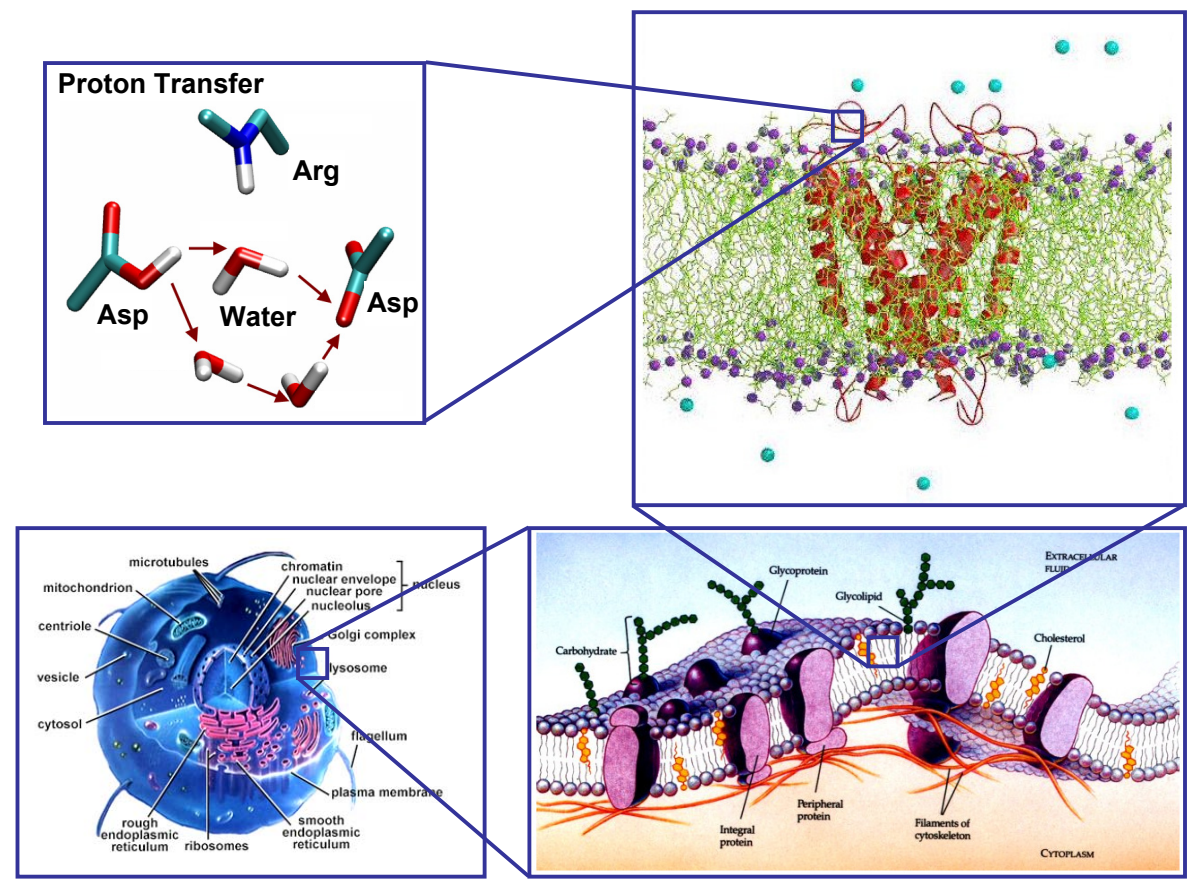

Figure 1.3: Multiscale and Multiphysics characteristics in bio systems. At electron size scale, chemical reactions, which are governed by huge QM effects (e.g., electron/proton transfer), regulate the molecular architecture of the protein for an effective functionality. At molecular scale, CM decribes the interactions, such as hydrophobic interaction, among the bio components of proteins, lipids, etc. Large scale motion of the membrane, such as endo- or exo-vesiculation, is in the regime of hydrodynamic descriptions, and this affects the dynamics of a cell. 
toward non-volatile flash memory devices, which has not yet achieved. Since the ring and backbone form a charge transfer complex, the local charge distribution (QM effect) strongly determines the free energy profiles for the rings shuttling. However, the time scale for the shuttling is order of $\mu \mathrm{s}$, which is beyond the available time scale of atomistic simulations. To handle such difficulties, we perform a "blue moon sampling technique" with MD simulations, which allows an effective sampling using a series of constraint MD simulations. The change of potential energy surface depending on the charge transfer amount is reflected on the MD simulations using various Mulliken charge populations from QM calculations according to the ring's location. The free energy barrier from the TTF station to the DNP station is evaluated as $19 \mathrm{kcal} / \mathrm{mol}$ when the system is neutral and $8-9 \mathrm{kcal} / \mathrm{mol}$ for the oxidation states +1 and +2 , and the the free energy barrier from the DNP station to the TTF station is $18 \mathrm{kcal} / \mathrm{mol}, 22-23 \mathrm{kcal} / \mathrm{mol}$, and $32-33 \mathrm{kcal} / \mathrm{mol}$ for the neutral and the oxidation states +1 and +2 , respectively. These values are quite comparable to the values that were obtained from various prior experiments, and greatly explain the microscopic ground for the on/off switching kinetics of the molecular switch.

In Chapter 4, the diffusion process of sodium ion in the aluminum-doped zeolite BEA system is studied. Understanding the ionic diffusion through a percolated pore of the zeolite is important for the application of the zeolite to proton exchange membranes for fuel cells (PEMFC). Especially, investigating the role of the confined water is vital because it provides a medium for transport of the ions. The amount of water swelled into the PEMFC depends on the macroscopic variables, such as pressure and temperature, where the PEMFC is working. Thus, combining the grand canonical Monte Carlo (GCMC) simulations (for the study of water uptake amount) with MD simulations (for the study of confined water structure), we investigate the effect of water on the sodium diffusion. We observe a first-order-like transition from the absorption isotherm, inferring that zeolite provides a hydrophobic environment. From MD trajectories, we observe the sodium ions diffused via a hopping mechanism among aluminum-doped sites (which are Brönsted acid sites). We figure out that, above $15 \mathrm{wt} \%$ hydration (good solvation regime), the solvation cage is easily formed, and dramatically increases sodium diffusion by reducing the hopping energy barrier by $25 \%$ from the value of 3.8 $\mathrm{kcal} / \mathrm{mol}$ observed in the poor solvation regime.

The second half (Chapters 5-7) discusses the multiscale and multiphysics simulation approaches for bio-systems. In particular, we scrutinize lipid systems, which have critical roles in cell structure, energy storage and metabolic control, using computational methods in collaboration with gas-phase experiments.

In Chapter 5, we study the ion mobility of a number of tertiary amine and quaternary ammonium cations in gas-phase. These tertiary amine and quaternary ammonium cations are related to the choline and its derivatives, which are precursors for lipids such as a phosphatidlcholine and sphingomyelin. For example, choline is oxidized to betaine, which is readily demethylated to yield 
$N, N$-dimethylglycine. Decomposition of choline yields trimethylamine and dimethylamine. Ion mobility in gas phase is determined by the collision of the ion with drift gas molecules. Utilizing the kinetic theory under the assumption of binary collision, the ion mobility in gas-phase is formulated as a complex integration of interaction terms between the ion and the drift gas molecule. We develop a methodology (referred to as modified trajectory (TJ) method) for the numerical integrations of complex interaction terms for the case where the ion drifts within the $\mathrm{N}_{2}$ buffer gas. Since the interaction terms depend on the electron distribution and molecular conformation, the gas phase structures and the charge distributions are investigated using QM calculations. Then, ion mobilities are calculated using the modified TJ method. The calculated ion mobility is in agreement with the experimental value, and it well explains the observed mass-mobility correlation of tertiary amine and quaternary ammonium cations, in terms of the asymmetry of the shape. In addition, computational numerical study allows us to identify the role of each interaction term (van der Waals interaction, ion-induced dipole interaction, ion-quadrupole interaction, charge-charge interaction) on the ion mobility at the border-line regime, which lies between the regime where the long-range interactions are dominant (small ion size) and the regime where the short-range interactions are dominant (large ion size).

In Chapter 6, we expand the ion mobility study of lipid precursors in Chapter 5 into the study of the intact lipids of phophatidycholines (PC). Since the PC contains a flexible acyl chain, the molecular fluctuation changes the mobility of the PC cation. Taking the molecular fluctuation effect into account, 200 conformations are sampled from the MD simulations, then, the average collision cross-section is computed using the modified TJ method for the calculation of ion mobility. Here, for the experimental section, a commercial traveling wave ion mobility spectrometry (TWIMS) has been employed. Our simulation results exhibit a good agreement with the experimental values. In particular, we reveal that the traveling wave applied in the experiments excites the ions. Since this excitation yields a less compact form of saturated PCs, while unsaturated PCs can not form such a stretched conformation due to the rigid double bonds, we observe distinct mass-mobility correlation lines for the saturated PC cations and for the unsaturated PC cations.

In Chapter 7, a model lung surfactant system composed of lipid monolayer with surfactant protein B (SP-B) is examined. Lung disease is the third leading cause of death in United States, and concerns about chronic respiratory tract disease increase significantly as the level of air pollution increases. Thus, chemical and physical comprehensions about the response of pulmonary surfactant (PS) system to ozone $\left(\mathrm{O}_{3}\right)$, one of the most common air pollutants, are required. Due to the interfacial characteristic of PS, the ozonolysis of PS can undergo different pathways according to the reaction environment, especially, depending on whether or not the reaction occurs under waterrich conditions. From the multiscale/multiphysics point of view, the ozonolysis is the result of the QM effect, but the reaction environment is determined by the location of each component, 
which is the outcome of the CM interactions among the molecules. In addition, the change of physical properties of PS (e.g., surface tension) is in the regime of larger-scale physics such as hydrodynamics. In this chapter, a complete study on the response of lipid monolayer to $\mathrm{O}_{3}$ is achieved at the air-liquid interface when $\mathrm{O}_{3}$ is introduced from the air. The chemical composition changes during the heterogeneous $\mathrm{O}_{3}$ reaction are analyzed using field induced droplet ionization (FIDI) mass spectrometry, which exhibits quite a different reaction pathway to the bulk-phase ozone reaction. Our MD simulation provides molecular level understanding and rationalization for the observed distinct reaction pathways.

Depending on the characteristics of systems and the objectives that we want to figure out, as exemplified in this thesis, distinct approaches are required to explain the actual observations from experiments. Based on the cases discussed here, we suggest various systematic strategies to overcome the limitations in time- and length-scales of the traditional monoscale approaches. In addition, due to the nature of multiscale and multiphysics phenomena, we expect that a concrete establishment of the fundamental multiscale modeling procedures will invoke interdisciplinary studies by tightly combining the developments occurring independently across fields. 


\section{Chapter 2}

\section{Negative Differential Resistance of Oligo (Phenylene Ethynylene) Self-Assembled Monolayer Systems: The Electric Field Induced Conformational Change Mechanism}

\subsection{Abstract}

We investigate here a possible mechanism for the room temperature Negative Differential Resistance (NDR) in the $\mathrm{Au} / \mathrm{AN}-\mathrm{OPE} / \mathrm{RS} / \mathrm{Hg}$ self-assembled monolayer (SAM) system, where AN-OPE = 2'amino, 5'-nitro oligo (phenylene ethynylene) and RS is a C14 alkyl thiolate. Kiehl and co-workers showed that this molecular system leads to NDR with hysteresis and sweep-rate-dependent position and amplitude in the NDR peak. To investigate a molecular basis for this interesting behavior, we combine first principles quantum mechanics (QM) and meso-scale lattice Monte Carlo (MC) methods to simulate the switching as a function of voltage and voltage rate, leading to results consistent with experimental observations. This simulation shows how the structural changes at the microscopic level lead to the NDR and sweep-rate dependent macroscopic $I$ - $V$ curve observed experimentally, suggesting a microscopic model that might aid in designing improved NDR systems.

\subsection{Introduction}

Esaki's discovery of the negative differential resistance (NDR) in Ge $p$ - $n$ diodes opened a new phase in semiconductor devices [1]. Since the NDR devices enable faster and more efficient circuits 
by reducing the number of transistors required, they have many applications such as high-speed integrated circuits and low-power memories. As the scale of electronic devices is reduced toward nano-scale sizes, it would be useful to demonstrate NDR in molecular electronic systems $[2,3,4,5$, $6,7,8,9,10,11,12,13]$.

Derivatives of oligo (phenylene ethynylene) (OPE) have been identified as a good candidate for the molecular junctions due to its rigid and good conducting (fully conjugated) characteristics $[9,11,12,13,14,15,16,17,18,19]$. Chen et al. reported that a SAM of amino-nitro substituted OPE (AN-OPE) between two Au electrodes exhibits NDR at $60 \mathrm{~K}$ with an applied voltage of $\sim 2 \mathrm{~V}$. The current-voltage $(I-V)$ curve is fully reversible, but the NDR peak decays as the temperature increases. This NDR has been rationalized by the electrochemical oxidation/reduction or resonant-tunneling mechanism $[15,16,17]$. Support for this oxidation/reduction mechanism was the correspondence between the threshold potential for the electrical conductance $(2.09 \mathrm{~V})$ and the electrochemical potential $(1.67 \mathrm{~V})[15,19]$.

The device showing NDR at room temperature (RT) is important for many practical applications $[20,21]$. However, the poor reproducibility in device construction and the limited device stability have hampered extensive study on NDR. Kiehl and co-workers showed that a SAM of AN-OPE deposited on an $\mathrm{Au}$ electrode coupled to a $\mathrm{Hg}$ electrode covered with a tetradecane-thiolate (RS) leads to a well-defined and stable NDR at RT [9]. In this system, a distinct sweeping-rate-dependency in the NDR hysteresis loop was observed for a bias voltage near $\sim 0.6 \mathrm{~V}$. The presence of hysteresis rules out the resonant tunneling mechanism $[5,6,7,8,10]$. Based on the observed hysteresis and a variety of detailed features of the characteristics, they proposed a charge capture (QC) mechanism to explain the macroscopic $I-V$ behavior. However, an atomistic level analysis of a charge capture process and other possible mechanisms has not yet been established.

Several studies suggest that the conformational change would be a plausible mechanism to explain hysteretic $I-V$ curve [14, 22], and the external electric field can induce conformational change of the molecule in the junction $[14,23,24,25,26]$. Especially, Donhauser et al. reported STM studies in which isolated AN-OPE molecules contained in a dodecane-thiolate SAM on the Au substrate show at least two states having different conductances [14]. They showed that the transition from the high-conductance state to the low-conductance state is switched by applying external electric field. However no detailed atomic level description of the mechanism was provided.

In this chapter, we use first principle theory to analyze the sweeping-rate-dependent hysteresis of NDR observed in Kiehl's system, focusing on the possibility of electric field based conformational changes. We find that this system has two states:

- a high-conductance phase stable at low field (planar structure) and

- a low-conductance phase stable at high field (twisted structure). 
The transition between the two phases is driven by the interaction between the external field and the molecular dipole moment of the middle phenylene ring in AN-OPE. This leads to consistent results with the charge capturing mechanism of Kiehl. Using coarse-grained Monte Carlo simulations, we investigated how such a molecular conformational change results in a sweep-rate-dependent hysteresis in the NDR as well as the detailed kinetics of transition.

\subsection{Simulation Details}

\subsubsection{Computational Details of QM Calculations}

To obtain the structures and energies of planar $(\mathrm{P})$ and twisted $(\mathrm{T})$ structures, we carried out QM calculations for a $(1 \times 1)$ periodic unit cell. We employed the Perdew-Burke-Ernzerhof $(\mathrm{PBE})$ generalized gradient approximation exchange-correlation density-functional with a plane wave basis set (540 eV cutoff), using the Vienna Ab initio Simulation Package (VASP) [27]. Only the gamma point is sampled in reciprocal space to reduce computational cost for this large system (67 atoms per periodic cell). For the electrodes, we used 3-layer of Au (111) surface with all gold atoms fixed at their bulk value $(a=2.8838 \AA$ ) during the geometry optimization steps. The OPE molecules were anchored on the $3 \times 3 \mathrm{Au}$ (111) surface through sulfur atoms with hexagonal packing.

To understand the local electronic structure and local interactions of the AN-OPE part, we performed non-periodic QM calculations of the isolated OPE with 3 connected Au atoms, using Jaguar package [28] with PBE exchange-correlation functional and LACVP** basis set. Using the geometries from VASP calculations, we carried out the single point calculations.

\subsubsection{Conductivities of $\mathrm{P}$ and $\mathrm{T}$ Conformations (NEGF Calculations)}

The $I-V$ performance of each conformation is calculated by combining Green's function theory with the DFT Hamiltonian that are determined from the SeqQuest calculation with PBE functional $[29,30]$. The current is calculated using following equation:

$$
I(v)=\frac{2 e}{h} \int_{-\infty}^{\infty} T(E, V)\left[f_{1}(E, V)-f_{2}\left(E, V_{2}\right)\right] d E
$$

where $T(E, V)$ is the transmission function for the AN-OPE SAM part.

\subsubsection{Coarse-Grained NN Interacting Hamiltonian}

The NN interacting Hamiltonian which describes the AN-OPE SAM is

$$
H=\sum_{i=1}^{N}\left(E_{i}^{\text {torsion }}(\chi)-D_{i} F\right)+\sum_{\mathrm{NN}} U_{i j}
$$


Table 2.1: AN-OPE Interaction Energies, $U_{i j}$, for Monte Carlo (MC) Calculations

\begin{tabular}{cccc}
\hline \hline & {$[10 \overline{10}]$ pair } & {$[01 \overline{10}]$ pair } & {$[11 \overline{2} 0]$ pair } \\
\hline$\{i, j\} \subset \mathrm{P}$ & -3.95 & -5.00 & -7.19 \\
$\{i, j\} \subset \mathrm{T}$ & -7.66 & -3.12 & -0.61 \\
$\{i \in \mathrm{P}, j \in \mathrm{T}\}$ or $\{i \in \mathrm{T}, j \in \mathrm{P}\}$ & -5.70 & -2.56 & 3.13 \\
\hline \hline
\end{tabular}

*All energy values are in $\mathrm{kcal} / \mathrm{mol}$.

where $E_{i}^{\text {torsion }}(\chi)$ is the internal torsional energy of the $i$-th AN-OPE molecule due to twisting the AN-OPE by an angle $\chi, D_{i}$ is the [0001] component of the dipole moment of the $i$-th AN-OPE, $F$ is the [0001] component of the external electric field, $U_{i j}$ is the intermolecular interaction energy between $i$-th and $j$-th AN-OPE, and summation over NN denotes that the summation is over nearest

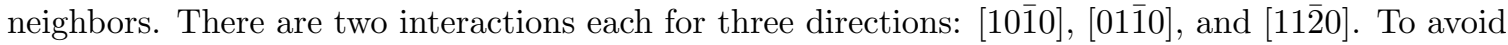
double counting, however, we used just one interaction per direction.

\subsubsection{Extracting NN Model Parameters from QM/FF Energies}

The $U_{i j}$ terms when $\{i, j\} \subset \mathrm{P}$ or $\{i, j\} \subset \mathrm{T}$ were determined from the Jaguar calculations (vide infra). In order to extract $U_{i j}$ when $\{i \in \mathrm{P}, j \in \mathrm{T}\}$ or $\{i \in \mathrm{T}, j \in \mathrm{P}\}$, we need to investigate interOPE interaction in the packed system from a larger simulation cell containing more than one OPE. Since this is too expensive for QM calculations (268 atoms), we used a simple DREIDING Force Field [31] (FF) in which the charges are based on QM. We considered six possible packings within a $(2 \times 2)$ unit cell:

- AP: all P's,

- AT: all T's,

- P3T1: 3 P's and 1 T,

- P2T2 ${ }_{10 \overline{1} 0}: 2$ P's and 2 T's aligned along [10̄̄0],

- $\mathrm{P} 2 \mathrm{~T} 2_{01 \overline{1} 0}: 2$ P's and 2 T's aligned along [01̄̄0],

- $\mathrm{P} 2 \mathrm{~T} 2_{11 \overline{2} 0}: 2$ P's and 2 T's aligned along $[11 \overline{2} 0]$,

- P1T3: $1 \mathrm{P}$ and 3 T's.

The FF energies are shown at Figures 2.1a and 2.1b for when no external electric field and $1.2 \mathrm{~V} / \AA$ external electric field is applied, respectively. Using FF energies under zero external field, the $U_{i j}$ terms when $\{i \in \mathrm{P}, j \in \mathrm{T}\}$ or $\{i \in \mathrm{T}, j \in \mathrm{P}\}$ were fitted. Values are in Table 2.1.

The torsional energy, $E_{i}^{\text {torsion }}$ is estimated from the energy versus twisting angle $\chi$ curve (Figure 2.2 ), leading the $0.01 \mathrm{kcal} / \mathrm{mol}$ per AN-OPE when $i \in P$, and $0.23 \mathrm{kcal} / \mathrm{mol}$ when $i \in T$. The 


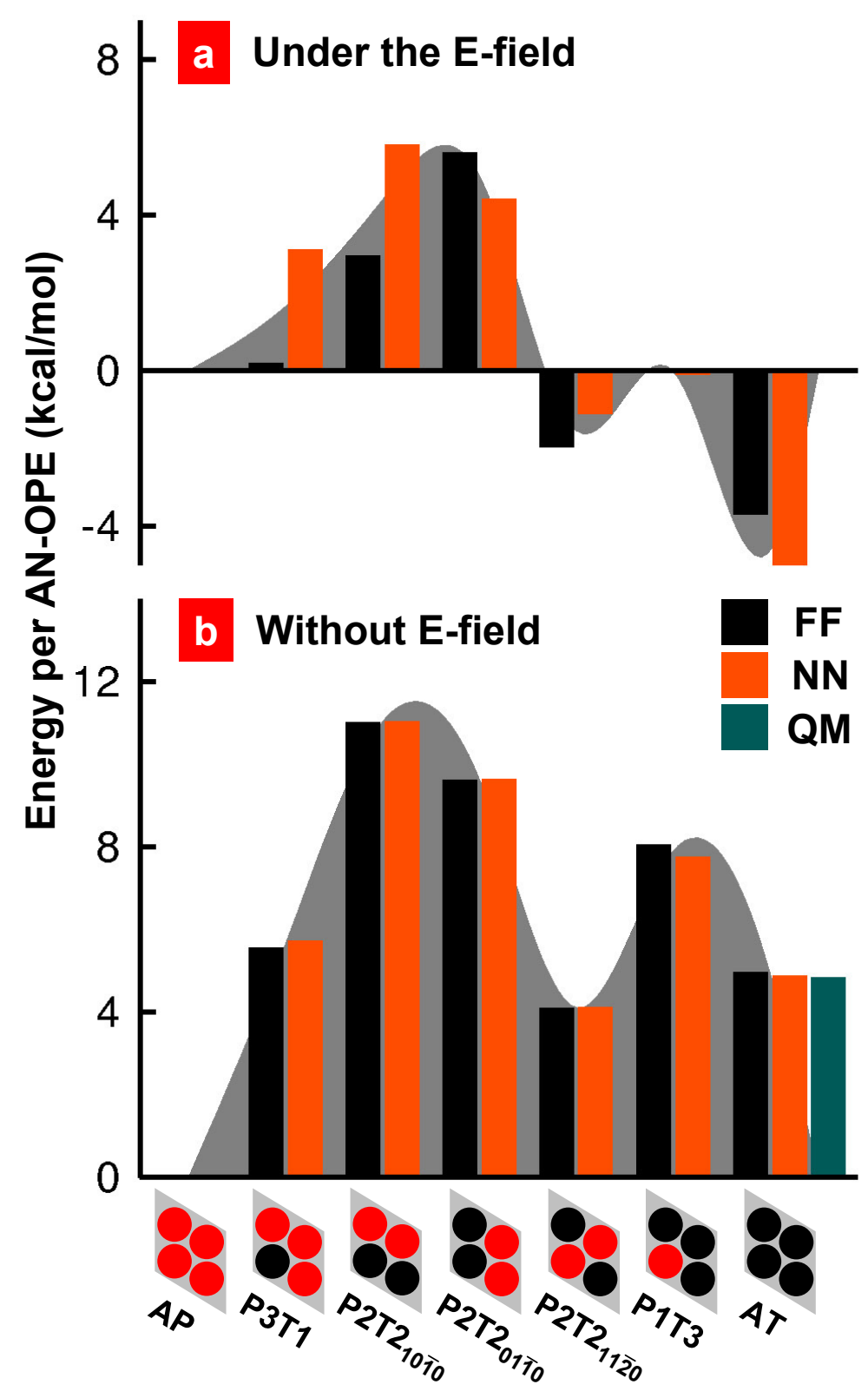

Figure 2.1: (a) Energies of various conformations of AN-OPE relative to the AP conformation computed with a $1.2 \mathrm{~V} / \AA$ external field from FF calculations (black histograms) and NN model calculations (orange histograms). (b) Energies of various conformations of AN-OPE relative to the AP conformation computed with no external field from FF calculations (black histograms), NN model calculations (orange histograms), and QM calculations (green histograms). 


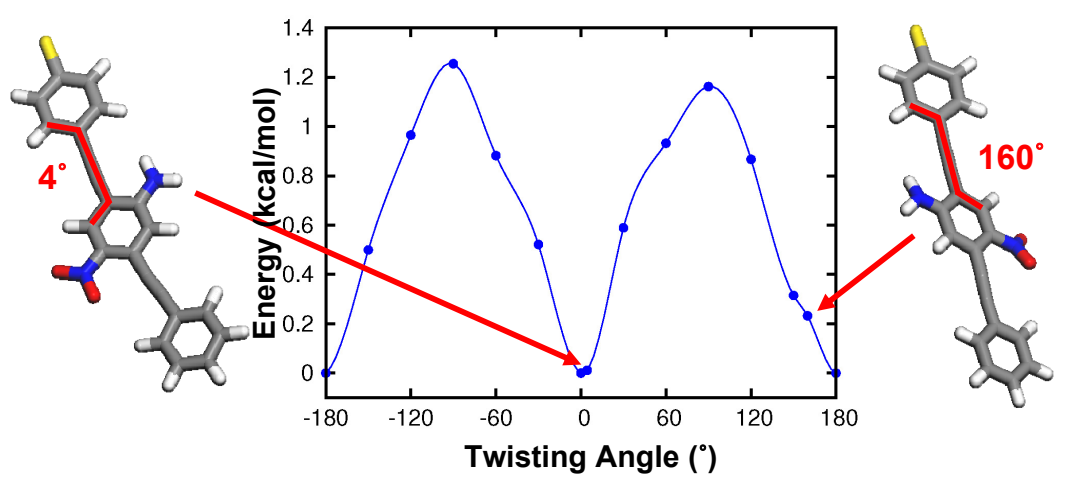

Figure 2.2: Torsional strain energy $E_{i}^{\text {torsion }}$ as a function of twisting angle $\chi$ from QM (PBE) on the isolated molecule. The energy at $\chi=4^{\circ}$ (corresponding to $\mathrm{P}$ ) is $0.01 \mathrm{kcal} / \mathrm{mol}$ higher than the ground state energy at $\chi=0^{\circ}$. The energy at $\chi=160^{\circ}$ (which corresponds to $\mathrm{T}$ ) is $0.23 \mathrm{kcal} / \mathrm{mol}$ higher than the energy at $\chi=0^{\circ}$, leading the $0.22 \mathrm{kcal} / \mathrm{mol}$ higher $E_{i}^{\text {torsion }}$ of $\mathrm{T}$ compared to $E_{i}^{\text {torsion }}$ of P.

$D_{i}$ values were optimized to fit the energies from Hamiltonian in Equation 2.2 to FF energies under $1.2 \mathrm{~V} / \AA$ external field. The fitted values are $D_{i}=5.48$ debye per AN-OPE for P and 7.30 Debye for $\mathrm{T}$, which are quite comparable to the dipoles from Mulliken charge analysis, $D_{i}=5.74$ Debye per AN-OPE for $\mathrm{P}$ and 7.03 debye for $\mathrm{T}$.

All MD simulations with DREIDING FF were performed using LAMMPS (large-scale atomic/ molecular massively parallel simulator) MD code from Plimpton at Sandia [32, 33]. The equations of motion were integrated using the velocity-Verlet algorithm [34], with a time step of $1.0 \mathrm{fs}$.

\subsubsection{Coarse-Grained MC Simulations}

The 2-dimensional rhombic MC simulation cell containing $(50 \times 50)$ lattice points with periodic boundary conditions. The MC simulations used the Metropolis algorithm (our own code). Each Monte Carlo Step (MCS) is defined as 2500 MC trials with a fixed external field applied in the $z$-direction. Our MC simulations are similar to the study of an Ising model in a time-dependent magnetic field [35].

\subsection{Results and Discussion}

\subsubsection{Two Conformations of AN-OPE}

Figure 2.3a shows the minimized conformation of AN-OPE determined from the periodic DFT calculation. The three phenylene rings are coplanar, forming a well-conjugated structure, which we label as $\mathrm{P}$ (for planar). Here the lowest ring is connected to the Au electrode via a thiolate group. The middle ring (containing the functional groups) has a twist angle of $\chi=4^{\circ}$ with respect to the 

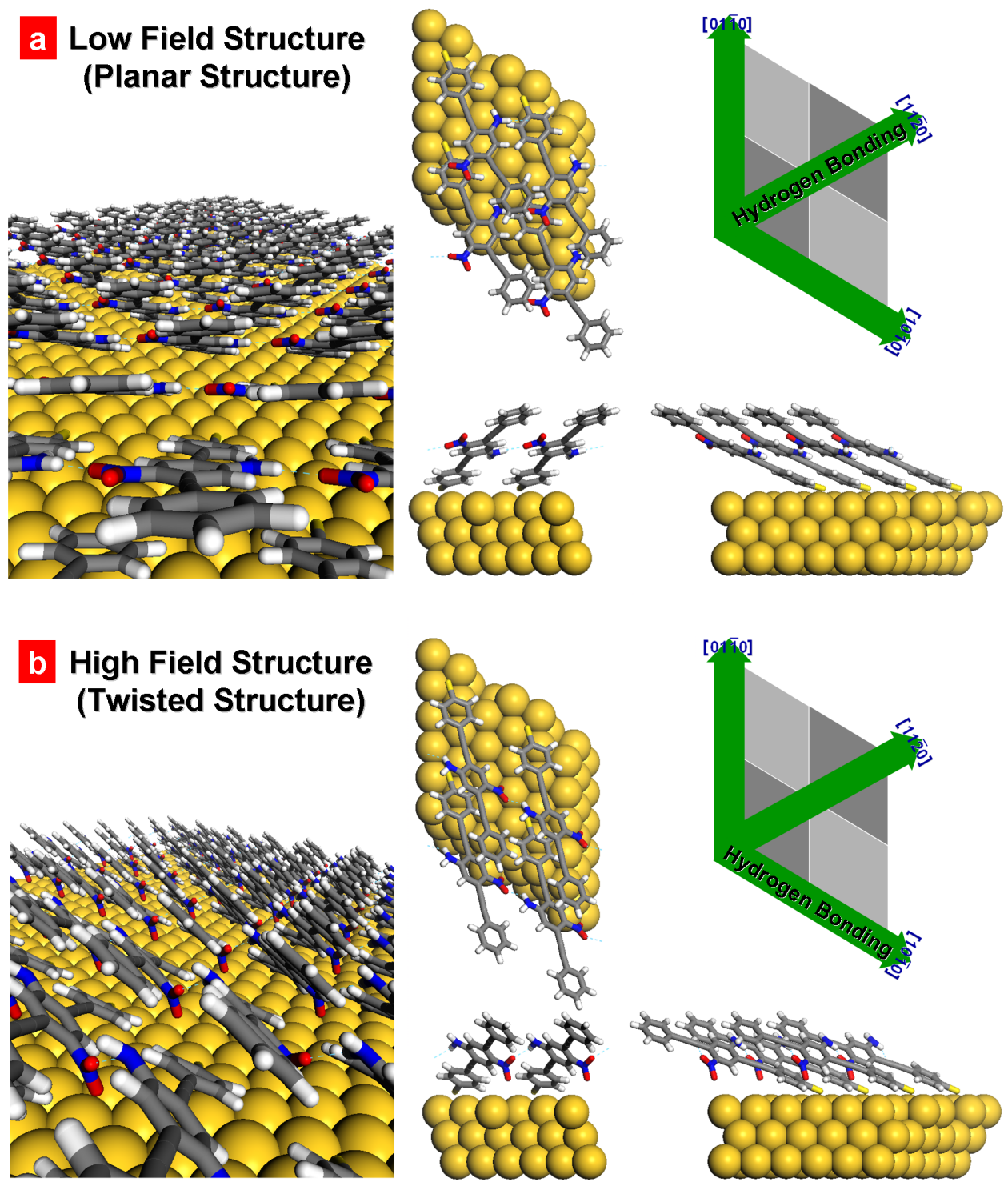

Figure 2.3: (a) Optimized geometry for the low field structure (P) of AN-OPE SAM. Here [0001] is the surface normal and the views are along $z$-axis (upper middle), $y$-axis (lower middle), and $x$-axis (lower right). The left picture is a perspective along the axis of one plane of molecules. The hydrogen bonding network is aligned along the [1120] direction. (b) Optimized geometry for the high field structure (T) of AN-OPE SAM. Here [0001] is the surface normal and the views are along $z$-axis (upper middle), $y$-axis (lower middle), and $x$-axis (lower right). The left picture is a perspective along the axis of one plane of molecules. The hydrogen bonding network is aligned along the [10 $\overline{1} 0]$ direction. 
bottom ring $\left(\chi=0^{\circ}\right.$ for the isolated OPE). This structure packs on the Au surface as $(3 \times 3)$, with the axis of the molecule along the [1100] direction, and a tilt angle $\theta=66^{\circ}$ from the $z$-axis. The adjacent amino and nitro groups form hydrogen bonding (HB) networks along the [1120] direction.

To understand the local electronic structure and local interactions of the organic molecular part, we performed a single point non-periodic QM calculation of an isolated OPE. The polar amino and nitro groups lead to a large dipole moment of 9.24 (7.22) debye with the $z$-axis component ([0001]) of 5.74 (3.55) debye, the component along [1100] (tilt direction) of 4.44 (3.35) debye, and the component along [1120] (HB direction) of 5.72 (5.32) debye. These dipole moments were determined from the analysis of Mulliken charges, while the values in parenthesis are from quantum mechanical wavefunctions.

The inter AN-OPE interaction energies on the SAM, $U_{i j}$ are determined from the difference between dimer energy and doubled monomer energy:

$$
U_{i j}=E_{2 \times(A N-O P E w / 3 A u)}-2 \times E_{A N-O P E w / 3 A u},
$$

where we included three $\mathrm{Au}$ atoms connected to the sulfur atom.

The value of $U_{i j}$ between two P's are shown at Table 2.1, especially, $U_{i j}$ along [11 $\left.\overline{2} 0\right]$ direction shows the largest stabilization energy of $-7.19 \mathrm{kcal} / \mathrm{mol}$, due to the HB interaction.

Figure $2.3 \mathrm{~b}$ shows the $\mathrm{T}$ (for twisted) conformation, which is $4.85 \mathrm{kcal} / \mathrm{mol}$ less stable than $\mathrm{P}$. The middle and terminal phenyl rings are rotated from the bottom one by $\chi=160^{\circ}$. Although we note that this twist angle is not stable for the isolated AN-OPE, it becomes meta-stable in the packed system (Figure 2.2). The rotation of the middle ring changes the direction of amino/nitro

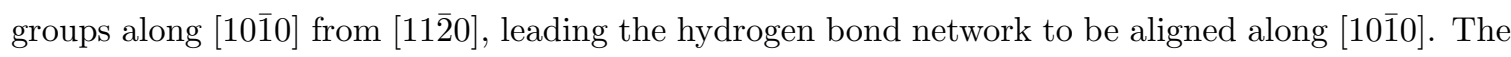
axis of the molecule is at $\theta=71^{\circ}$ from the $z$-axis, which makes the $\mathrm{T}$ lies more down than the $\mathrm{P}$. The height of the terminal phenyl ring of $\mathrm{T}$ is $7.60 \AA$ while that of $\mathrm{P}$ is $8.73 \AA$ from the Au surface. The lower height of $\mathrm{T}$ is comparable to the Donhausers observations from the STM experiment that exhibits $\sim 3 \AA$ lower height of low conductance phase to higher conductance phase [14].

The dipole moment of $\mathrm{T}$ is 8.71 (6.88) debye with the component along [0001] of 7.04 (4.62) debye, a component along [11̄00] (tilt direction) of $-3.32(-3.40)$ debye and a component along [1010] (HB direction) of 5.12 (5.10) debye.

The value of $U_{i j}$ between two T's shown at Table 2.1. For T conformation, $U_{i j}$ along the [1010] direction (HB network direction) is the most stable with the value of $-7.66 \mathrm{kcal} / \mathrm{mol}$.

\subsubsection{Electrical Conductivities of $\mathrm{P}$ and $\mathrm{T}$}

The electrical conductivity, $\sigma$, was predicted using non-equilibrium Green's function (NEGF) theory for $\mathrm{P}$ and $\mathrm{T}$ structures with $\mathrm{QM}$ on the $(1 \times 1)$ unit cell. These calculations partition the tunnel- 
a Planar Structure
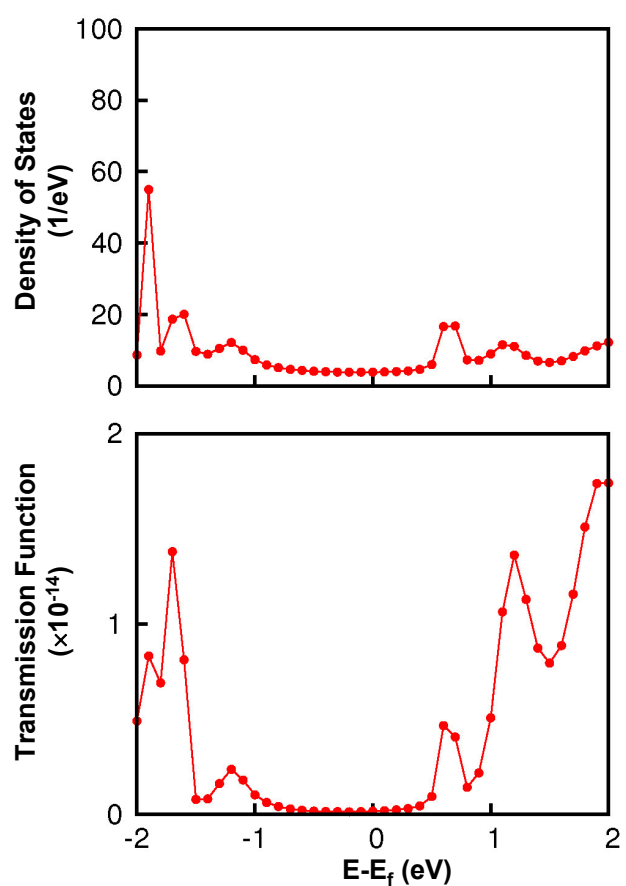

b Twisted Structure
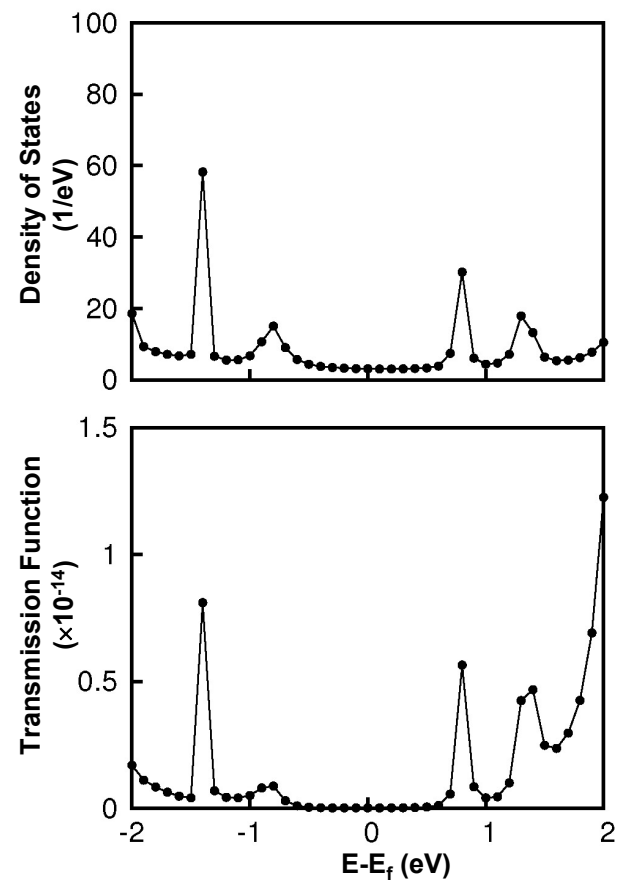

Figure 2.4: Density of state (DOS) and transmission function $T(E)$ of $\mathrm{P}$ structure (a) and $\mathrm{T}$ structure (b). The top electrode is located $12 \AA$ above from the bottom electrode.

ing Hamiltonian using the Gaussian basis function representation. The semi-infinite electrode is calculated interactively using 3 explicit layers of $\mathrm{Au}[29]$.

The experiments use a top $\mathrm{Hg}$ electrode covered with a tetradecane-thiolate (RS), the atomic structure of which is not certain due to the amorphous character of the $\mathrm{Hg}$ electrode and the fluctuations in the alkyl thiol at room temperature. Instead, our calculations use a second 3-layer of $\mathrm{Au}$ (111) surface $12 \AA$ above the bottom electrode. We also tested placing the top electrode in contact with the OPE, which $20 \AA$ above the bottom electrode.

The density of states (DOS) with the transmission function $T(E)$ were computed (Figures 2.4 and 2.6), and these are used to obtain the $I-V$ curve and the $\sigma-V$ curve (Figures 2.5 and 2.7). Over the range of 0 to $1.5 \mathrm{~V}$, we see that the average ratio of $\sigma_{P}$ to $\sigma_{T}$ is 10 for the thickness of $12 \AA$, and 163 for the thickness of $20 \AA$. Since the experimental value is $\sim 13$ fold larger $\sigma_{P}$ than $\sigma_{T}$, we adopted the case of $12 \AA$.

The calculated $I-V$ curves lead correctly to a smaller $\sigma$ for the high field stable phase. The difference in $\sigma$ is explained by two factors:

1. the loss of $\pi-\pi$ orbital overlap due to the rotation of the middle phenyl ring (which contributes to the conductivity through the molecules) and 

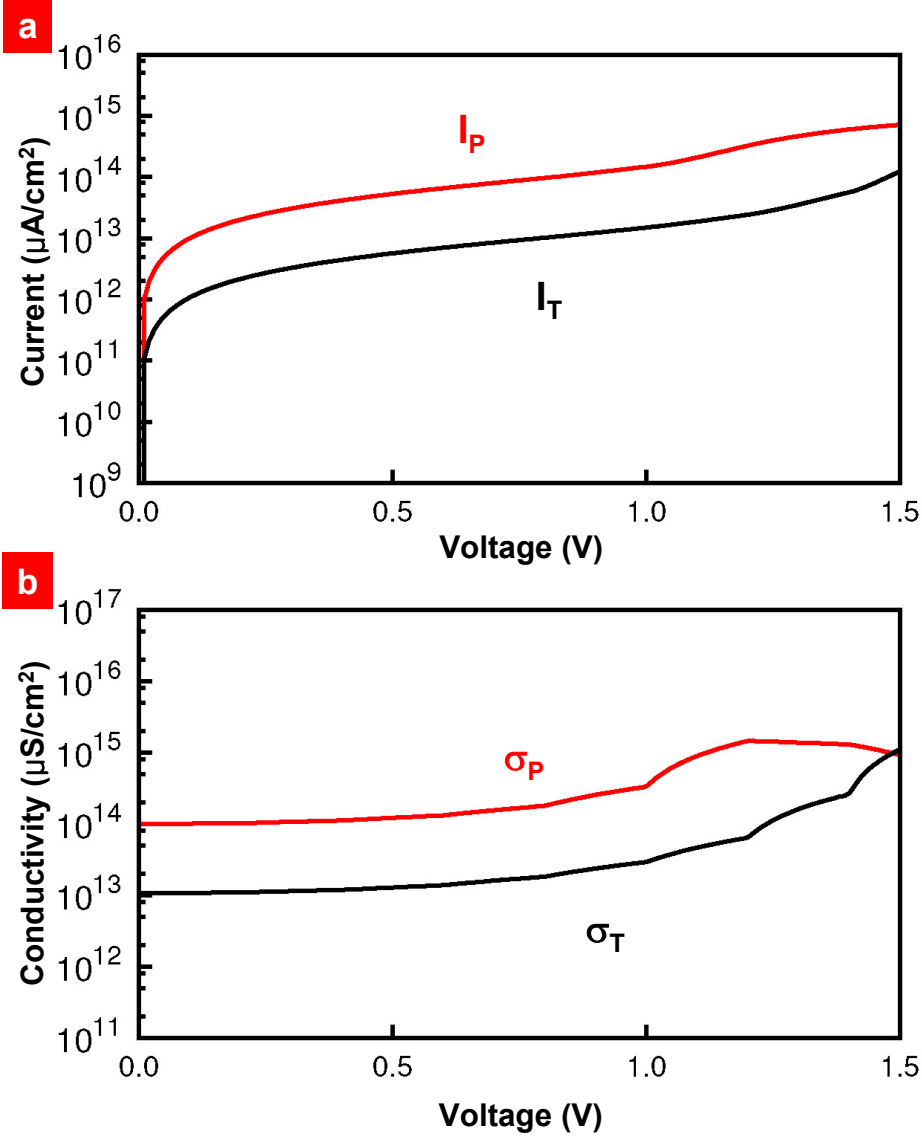

Figure 2.5: (a) Current through $\mathrm{P}$ conformation, $I_{P}$ and current through $\mathrm{T}$ conformation, $I_{T}$ versus the bias voltage $V$ determined from the NEGF calculations using the DOS and T(E) of Figure 2.4. (b) Conductivity of $\mathrm{P}$ conformation, $\sigma_{P}$ and conductivity of $\mathrm{T}$ conformation, $\sigma_{T}$ versus the bias voltage $V$ determined. The top electrode is located $12 \AA$ above from the bottom electrode. $\sigma_{P}$ is $\sim 10$ times larger than $\sigma_{T}$. 
a Planar Structure
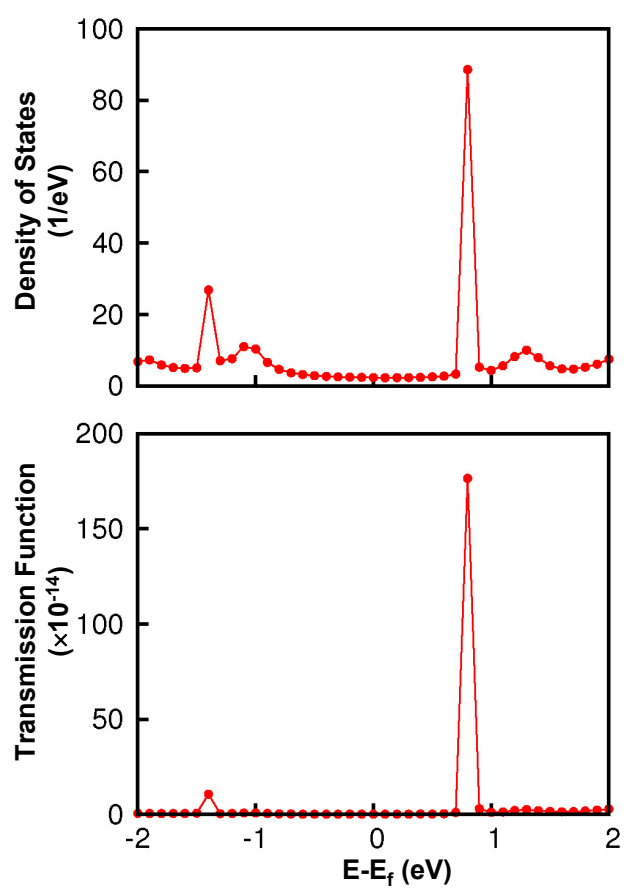

b Twisted Structure
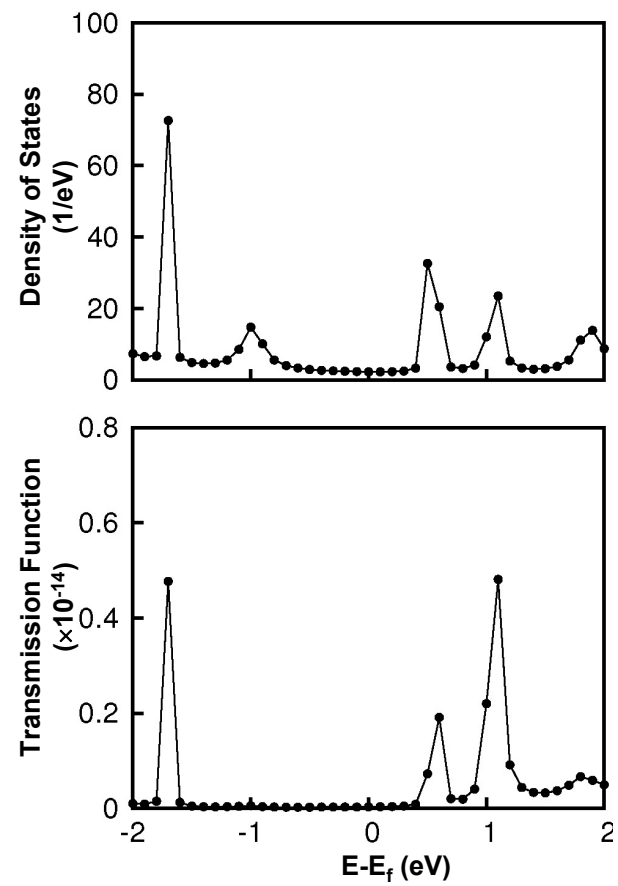

Figure 2.6: Density of state (DOS) and transmission function $T(E)$ of $\mathrm{P}$ structure (a) and $\mathrm{T}$ structure (b). The top electrode is located $20 \AA$ above from the bottom electrode.

2. the increase of vacuum distance due to the lower height of $\mathrm{T}$ phase.

Previous studies showed that the rotation of the ring by $\chi$ reduces the conductivity by factor of $\cos ^{4} \chi$ (Figure 2.8) [36]. Thus, using the QM minimized structures, which are twisted by $4^{\circ}$ and $160^{\circ}$, yield an average ratio of $\sigma_{P}$ to $\sigma_{T}$ by $\sim 1.3$ times. However, considering the distribution of angles from the $\mathrm{MD}$, we find an average ratio of $\sigma_{P}$ to $\sigma_{T}$ of $\sim 3.5$ times. (Detals are discussed in Appendix B.)

The minimized structures for $\mathrm{P}$ and $\mathrm{T}$ lead semi-log plots of $I-V$ curves (Figure 2.5) to show similar slopes for both phases, in disagreement with experiment showing a slope ratio of $\sim 3.2$. From previous study [36], we found that the slope of the semi-log plot of $I-V$ curve strongly depends on the $\chi$, and it decreases until almost zero when the molecule twisted with $\chi=90^{\circ}$ (Figure 2.8a). This is a reasonable result since the slope of semi-log plot is related to the height of tunneling barrier, $\Phi_{B}$ which highly dependeds on the $\pi-\pi$ orbital overlap. Therefore, consideration of the twisted structure from MD simulations, which has a siginificant population near $90^{\circ}$, well explains the distinct difference between the slopes of semi-log $I-V$ plots for both structures observed in the experiment. 

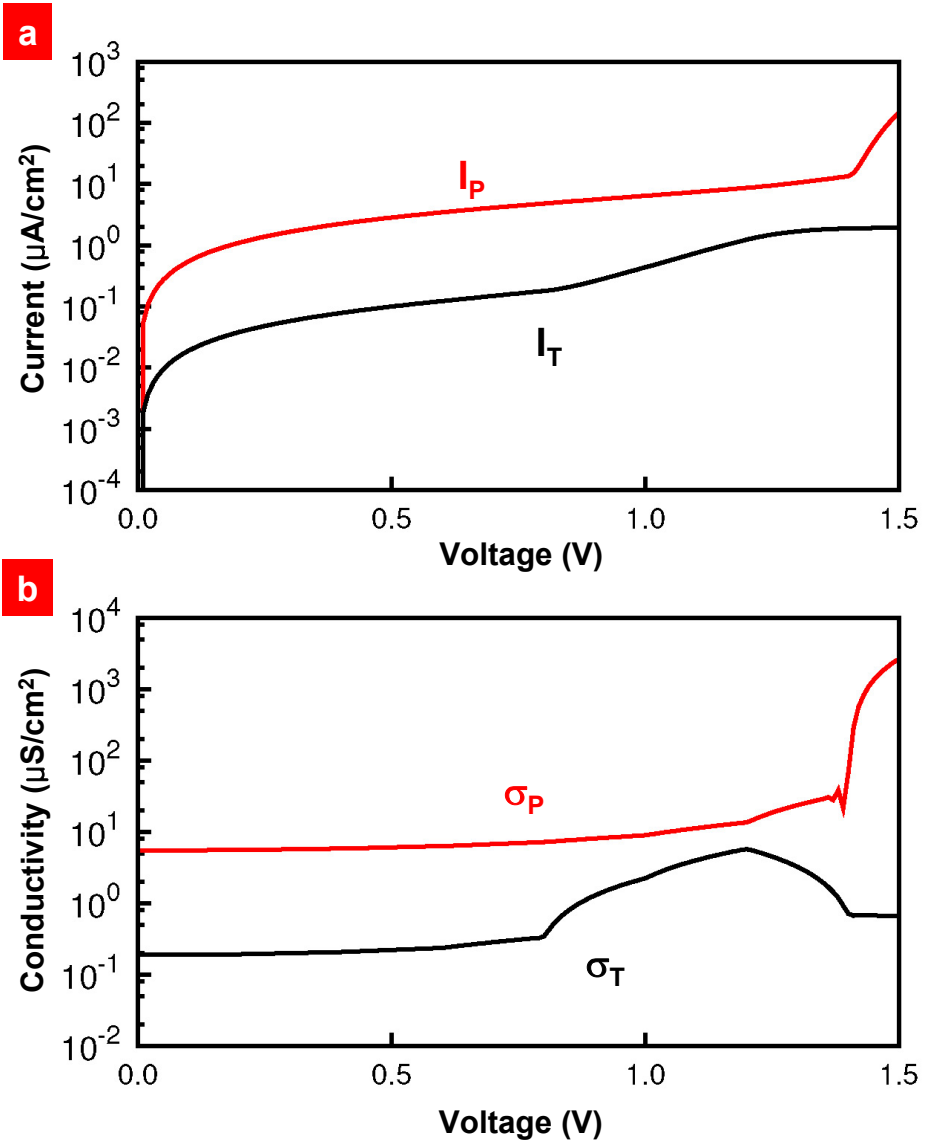

Figure 2.7: (a) Current through $\mathrm{P}$ conformation, $I_{P}$ and current through $\mathrm{T}$ conformation, $I_{T}$ versus the bias voltage $V$ determined from the NEGF calculations using the DOS and T(E) of Figure 2.6. (b) Conductivity of $\mathrm{P}$ conformation, $\sigma_{P}$ and conductivity of $\mathrm{T}$ conformation, $\sigma_{T}$ versus the bias voltage $V$ determined. The top electrode is located $20 \AA$ above from the bottom electrode. $\sigma_{P}$ is $\sim 163$ times larger than $\sigma_{T}$. 

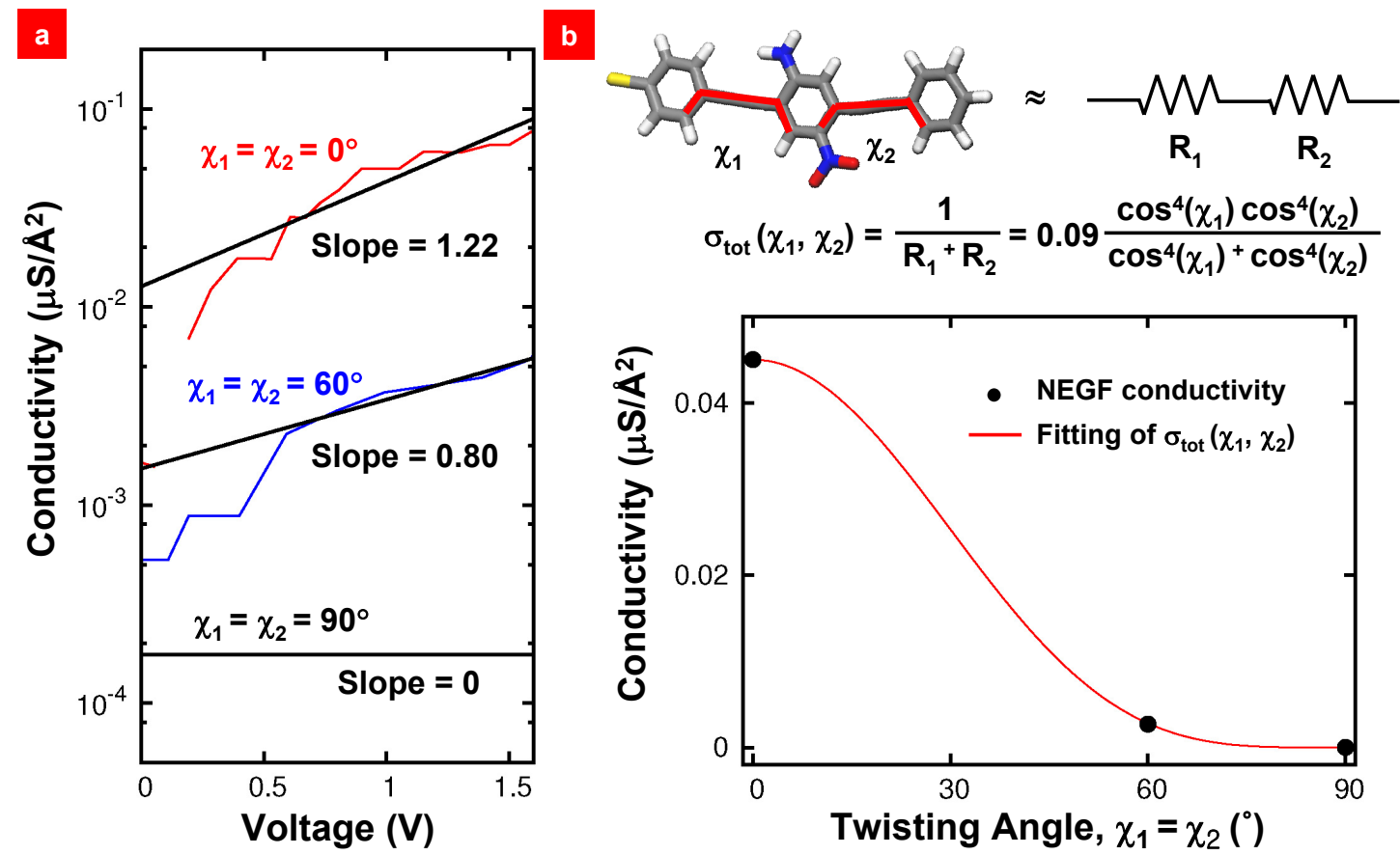

Figure 2.8: Conductivities depending on the twisting angle are extracted from the previous study [36], which are the results from NEGF calculations. (a) Semi-log plots of $I-V$ curves shows that the slope decrease as the twist angle approaches to $90^{\circ}$, which means that the tunneling barrier from the bottom electrode to top electrode, $\Phi_{B}$ increases as the the $\pi$ - $\pi$ orbital overlap decreases. (b) Since the conductivity is dominated by the $\pi-\pi$ orbital overlap, we assumed that the conductivity between two phenyl rings, $\sigma_{i}=1 / R_{i}$ is proportional to $\cos ^{4}\left(\chi_{i}\right)$. From simple calculation leads the total conductivity tot to be proportional to the $\cos ^{4}\left(\chi_{1}\right) \cos ^{4}\left(\chi_{2}\right) /\left(\cos ^{4}\left(\chi_{1}\right)+\cos ^{4}\left(\chi_{i}\right)\right)$, which shows good agreement with the NEGF results. 


\subsubsection{Response to Constant External Field}

In order to describe the structural rearrangement process of the AN-OPE SAM, we use a $(50 \times 50)$ unit cell containing 2500 AN-OPE molecules. To describe the dynamics of such a large simulation cell, we developed a simplified nearest neighbor (NN) interaction Hamiltonian with the model parameters extracted from QM and Force Field (FF) energies (Figure 2.1). Important feature of the NN model is that the energetically favorable state changes from $\mathrm{P}$ to $\mathrm{T}$ as the external electric field increases, due to the higher dipole moment along [0001] of the T phase. In this model, the critical field for which the energies of both states are same is $F_{c}=0.56 \mathrm{~V} / \AA$.

We simulated the response of the SAM of the P's to the $1.2 \mathrm{~V} / \AA$ external field during $1.2 \times 10^{6}$ Monte Carlo steps (MCS) at $T=300 \mathrm{~K}$. Figures 2.9a and 2.9b show the time evolution of the system starting with all P's and ending with all T's and the population change with the electrical conductivity change during this P-to- $\mathrm{T}$ transition, respectively. There were no $\mathrm{T}$ states until just before 55, $184 \mathrm{MCS}$, but within the next 15 steps, half of the neighbors along [1120] have transformed, and after another 15 steps the entire [1120] line is transformed to the T. Then, by 405, 907 MCS, totally 19 [11 $\overline{2} 0]$ lines have transformed, which are all neighbors to the original one. Then, at 486, 389 MCS, we see that a second [1120] swath has nucleated. By 697,272 MCS, these two have grown to 28 and 7 adjacent lines but still just two swathes, and from 697,273 MCS, they are merged into one swath. Finally, by 1,008, 706 MCS the full system is transformed to T. Along with the decrease of the $\mathrm{P}$ population, the total conductivity through the SAM also decreases.

The analysis of the snapshots demonstrates that the time to complete the transformation of each

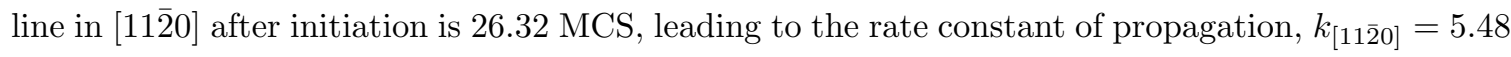
$\AA / \mathrm{MCS}$. The $k_{[11 \overline{2} 0]}$ shows almost no dependence on the temperature (Figure 2.10a, top), which infers that the energy barrier for the propagation is zero or quite negligible. Thus, once one ANOPE is switched as a nucleation, the transformation propagates quickly along the [112̄0] line, which is quite reasonable in terms of the energetic stability of $\mathrm{P} 2 \mathrm{~T} 2_{11 \overline{2} 0}$ (Figure 2.1). Then, another nucleation occurs for subsequent transformation of another [11 $\overline{2} 0]$ line. Typically this subsequent transformation takes place right next to the precedent transformed line in the [1]100] direction.

Therefore, the nucleation is the key step governing the time scale of the transition. The middle panel of Figure 2.10a shows the probability, $P(t)$ of exhibiting no nucleation until time $t$. To obtain the nucleation time $(\tau)$ when we have a transformed [11 $\overline{2} 0]$ line already, the nucleation events initiated next to [112̄0] line are analyzed among the snapshots. The nucleation process is found to be a Poisson process, in which $P(t)$ decays exponentially with time. The value of $\tau$ was obtained as 5,583 MCS by fitting of $P(t)$.

To obtain the nucleation time $\left(\tau_{0}\right)$ in the absence of [11 $\left.\overline{2} 0\right]$ line, we carried out additional 50 simulations to find when the first $\mathrm{P}$ transforms to the $\mathrm{T}$, showing that this process proceeds as a Poisson process with $\tau_{0}=274,193 \mathrm{MCS}$ that is $\sim 50$ time larger than $\tau$ (Figure 2.10a, bottom 


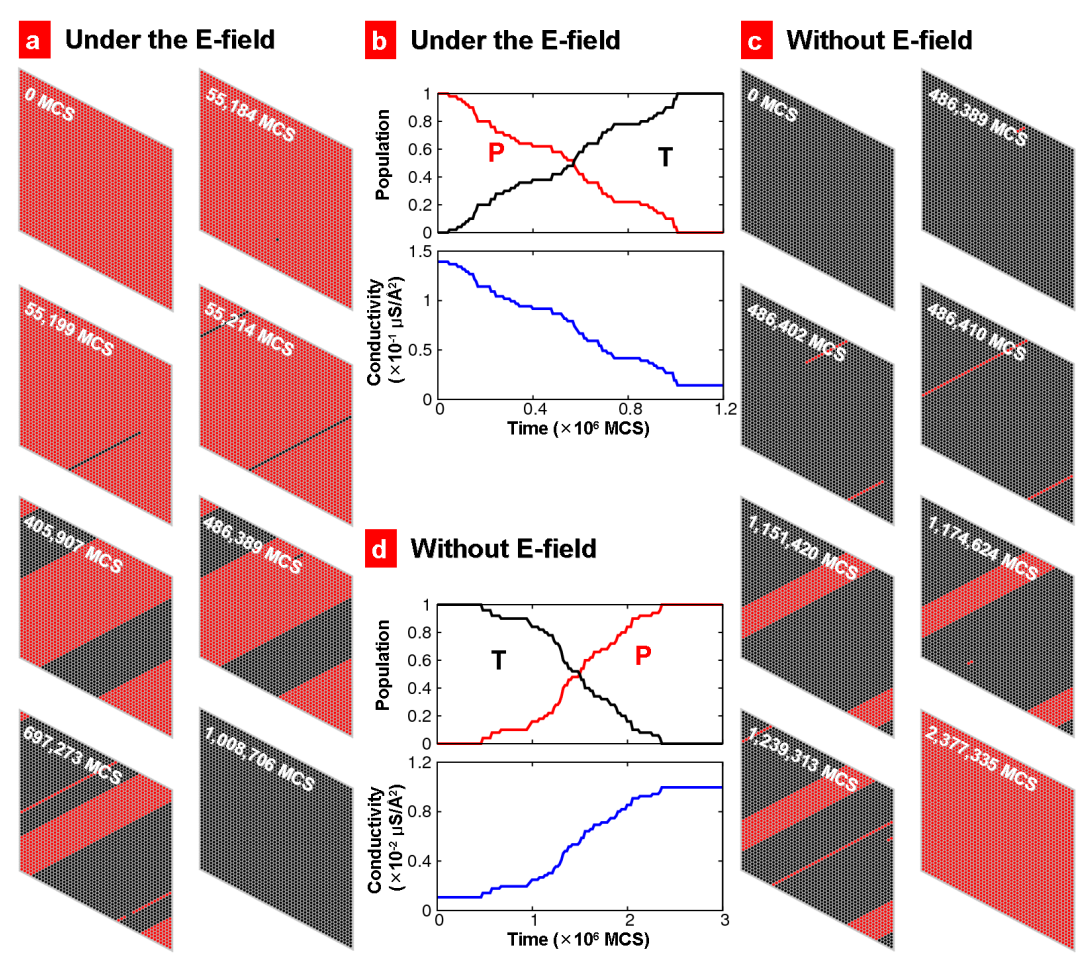

Figure 2.9: (a) Snapshots during P-to-T transition from MC simulation with $1.2 \mathrm{~V} / \AA$ external field. The first nucleation is occurred at 55, $184 \mathrm{MCS}$, then, the [1120] line propagates until 55, 214 MCS. More nucleation and propagation along [1120] are taken place, and finally, the full system is transformed by 1,008, 706 MCS. We note that increased bias voltage yields a faster P-to-T transition. (b) Time dependence of $\mathrm{P}$ and $\mathrm{T}$ populations and electrical conductivity through the SAM during P-to-T transition. (c) Snapshots during T-to-P transition from MC simulation without an external field. The first nucleation is occurred at 486,389 MCS, then, the [112̄0] line propagates until 486, 410 MCS. More nucleation and propagation along [1120] are taken place, and finally, the full system is transformed by 2,377,335 MCS. (d) Time dependence of $\mathrm{P}$ and $\mathrm{T}$ populations and electrical conductivity through the SAM during T-to-P transition. 
a P-to-T transition
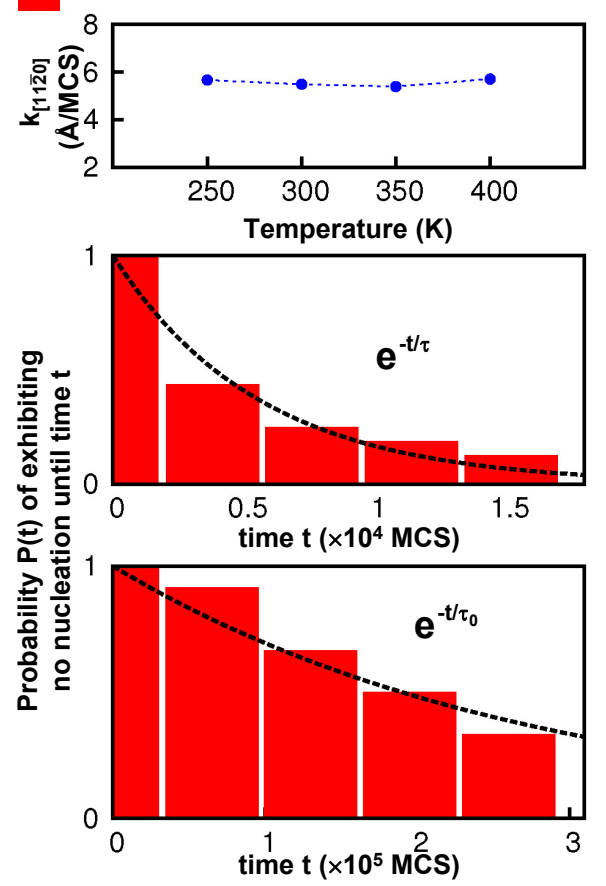

b T-to-P transition
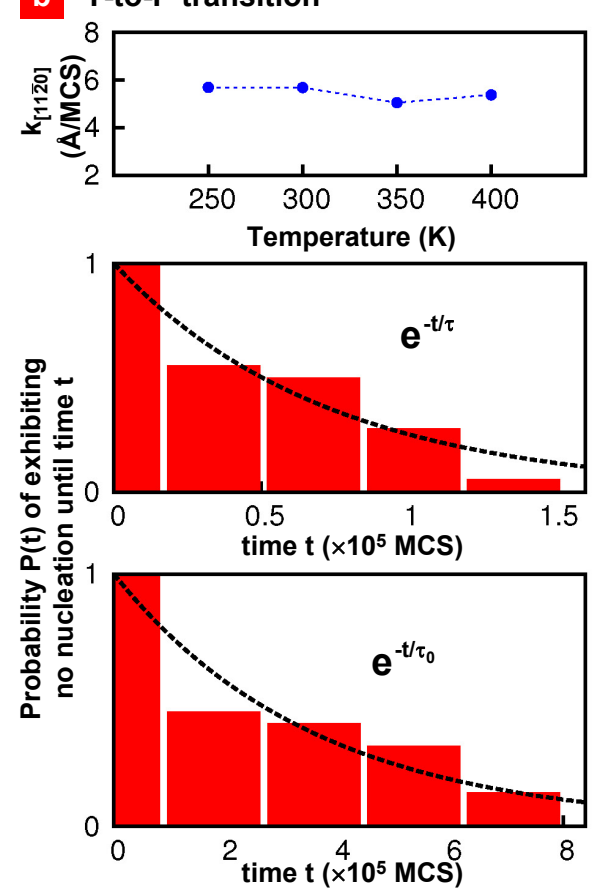

Figure 2.10: (a) P-to-T transition; (b) T-to-P transition; Top panels show the temperature de-

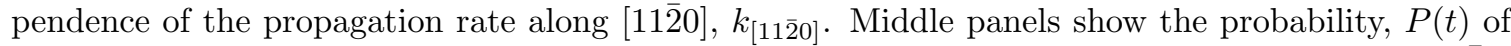
exhibiting no nucleation by time, $t$ for the case when the nucleation is initiated next to another $[11 \overline{2} 0]$ line. The dotted lines is an exponential fit of $P(t)$. This leads to a nucleation rate of $\tau=5,583 \mathrm{MCS}$ for the P-to-T transition and $\tau=72,926$ MCS for the T-to-P transition. Bottom panels show the probability, $P(t)$ of exhibiting no nucleation by time, $t$ for the case when the nucleation is initiated in the absence of next [112̄0] line. The exponential fit leads to a nucleation rate of $\tau_{0}=274,193$ MCS for the P-to-T transition and $\tau_{0}=357,135$ MCS for the T-to-P transition. 
panel).

Figures $2.9 \mathrm{c}$ and $2.9 \mathrm{~d}$ show the time evolution of the system with no external field starting with all T's and ending with all P's and the population change with the electrical conductivity change during this T-to-P transition, respectively. The overall process is quite similar to that of $\mathrm{P}$-to- $\mathrm{T}$ transition except for the detailed numbers. The first nucleation occurred at 486, $389 \mathrm{MCS}$, and then, the neighbors along $[11 \overline{2} 0]$ showed fast transition to $\mathrm{P}$ within 21 MCS. While this swath is growing along [1ํㅣㅇㅣ direction, the second and the third nucleation without next transformed line occurred at 1,174,624 MCS and 1,239,313 MCS, respectively. Finally, the full system is transformed to P by $2,377,335$ MCS. During the transition, the total conductivity through the SAM increases along with the decrease of the $\mathrm{P}$ population.

During T-to-P transition, the average time to complete each line of [1120] growth is 25.38 MCS, leading to $k_{[11 \overline{2} 0]}=5.68 \AA / \mathrm{MCS}$ with no temperature dependency (Figure 2.10b, top panel).

The nucleation times are studied in the same manner with the P-to-T transition. The nucleation process follows Poisson process with the $\tau=72,926 \mathrm{MCS}$ and $\tau_{0}=357,135 \mathrm{MCS}$ in the presence and the absence of the next transformed [1120] line, respectively (Figure 2.10b, middle and bottom panels).

The interaction between $\mathrm{P}-\mathrm{T}$ is smaller than the interaction between $\mathrm{P}-\mathrm{P}$ or $\mathrm{T}-\mathrm{T}$ by $\sim 8.6$ $\mathrm{kcal} / \mathrm{mol}$ in average (Table 1), due to the loss of HB or less-favorable van der Waals interaction caused by the packing of two different conformations. This infers that the boundary of $[11 \overline{2} 0]$ line is energetically less stable, leading a fast transformation at the boundary. This well explains the smaller $\tau$ than $\tau_{0}$.

We also found $\tau$ during P-to-T transition is smaller than $\tau$ during T-to-P transition (which is responsible to that NDR is not shown during the backward sweep). This is because the formation of T-T HB network is accompanied with the expansion of [11 $\overline{2} 0]$ swath along [1ํㅣㄹ during the P-to-T transition, while the loss of T-T HB network is accompanied during the T-to-P transition.

\subsubsection{NDR for Time Dependent Electric Field}

Applying a time dependent external field, we calculated the response of the system to voltage sweeps at various sweep rates. For each sweep, the magnitude of the external field was increased linearly until $F=1.4 \mathrm{~V} / \AA$ (corresponding to $1.5 \mathrm{~V}$ bias voltage in forward sweep) and then it was decreased at the same rate until the field was $0 \mathrm{~V} / \AA$ (corresponding to $0 \mathrm{~V}$ bias voltage in backward sweep). (Appendix D discusses the conversion factor between the external electic field and the bias voltage.) The sweep rates were $1 \times 10^{-8} \mathrm{~V} / \mathrm{MCS}, 4^{-8} \mathrm{~V} / \mathrm{MCS}$, and $2 \times 10^{-7} \mathrm{~V} / \mathrm{MCS}$. The resultant $I$ $V$ curves are shown in Figure 2.11a. Although the current drops dramatically at sufficiently high voltage, similar for all cases, we found that faster sweep let the systems stay in the P phase at higher voltage, which is in a good agreement with the experimental observation. Clearly, the simulations 


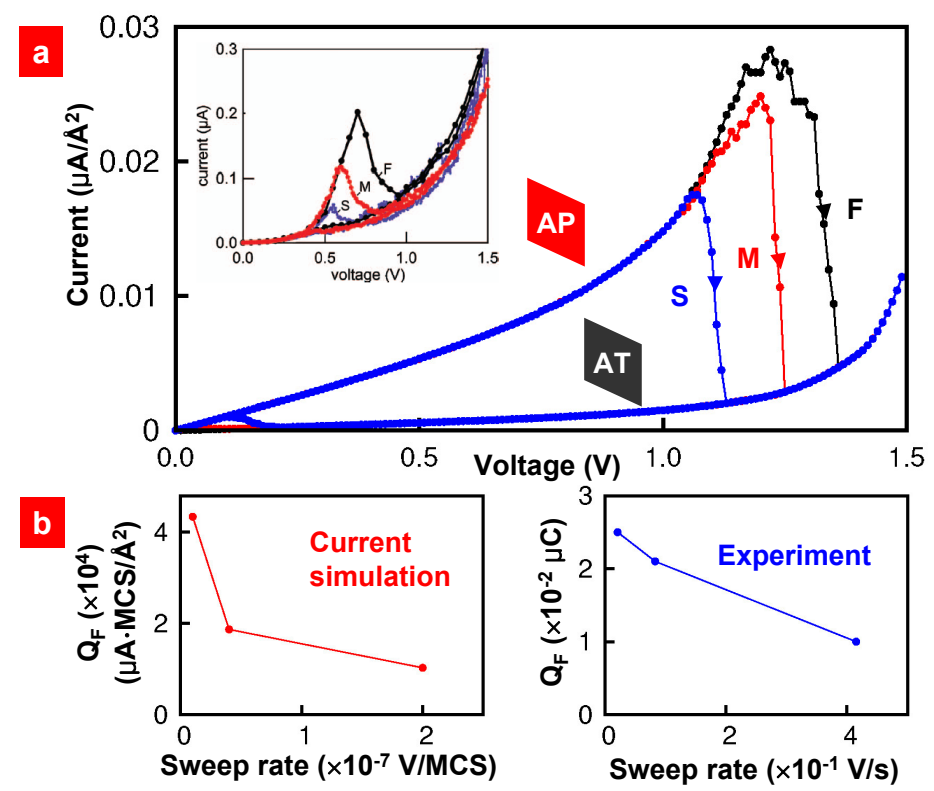

Figure 2.11: (a) Current-voltage $(I-V)$ curves calculated at $300 \mathrm{~K}$ from MC simulations combined with the $I-V$ results of the Green's function calculations. Results for three sweeping rates are shown: $\mathrm{S}$ (blue line): $1 \times 10^{-8} \mathrm{~V} / \mathrm{MCS}, \mathrm{M}$ (red line): $4 \times 10^{-8} \mathrm{~V} / \mathrm{MCS}$, and F (black line): $2 \times 10^{-7} \mathrm{~V} / \mathrm{MCS}$. Inset is the experimental $I-V$ curves from the reference [9] with 3 different sweeping rates: $\mathrm{S}$ (blue line) : $21 \mathrm{mV} / \mathrm{sec}, \mathrm{M}$ (red line) : $83 \mathrm{mV} / \mathrm{sec}$, and $\mathrm{F}$ (black line) : $415 \mathrm{mV} / \mathrm{sec}$. (b) The sweep rate dependence of the integral of the current from the peak to the valley during NDR, $Q_{F}$ from current simulation (left panel) and experiment [9] (right panel). 
show both the NDR phenomena and hysteretic behavior with sweep rate dependence as observed experimentally.

We found that the P-to- $\mathrm{T}$ transition voltage is located at $1.1-1.4 \mathrm{~V}$, depending on sweep rate. This can be compared to the experimental results in which the transition is completed by 0.6 to 1.0 V. During the backward sweep, the simulations found that the T state transforms back to $\mathrm{P}$ at $\sim 0.2 \mathrm{~V}$ bias voltage with slower sweep rates.

To determine the total charge associated with the NDR region, we integrated the current from the peak to the valley in Figure 2.11a. This amount of charge $\left(Q_{F}\right)$ flowing through the junction during NDR, is an important physical quantity that characterizes the QC model [9]. The calculated $Q_{F}$ values are

- $Q_{F}=4.34 \times 10^{4} \mu \mathrm{A} \cdot \mathrm{MCS} / \AA^{2}\left(\sim 1.76 \times 10^{6} \mathrm{e} / \mathrm{AN}-\mathrm{OPE}\right)$, for sweep rates of $1 \times 10^{-8} \mathrm{~V} / \mathrm{MCS}$,

- $Q_{F}=1.87 \times 10^{4} \mu \mathrm{A} \cdot \mathrm{MCS} / \AA^{2}\left(\sim 7.57 \times 10^{5} \mathrm{e} / \mathrm{AN}-\mathrm{OPE}\right)$, for sweep rates of $4 \times 10^{-8} \mathrm{~V} / \mathrm{MCS}$, and

- $Q_{F}=1.03 \times 10^{4} \mu \mathrm{A} \cdot \mathrm{MCS} / \AA^{2}\left(\sim 4.17 \times 10^{5} \mathrm{e} / \mathrm{AN}-\mathrm{OPE}\right)$, for sweep rates of $2 \times 10^{-7} \mathrm{~V} / \mathrm{MCS}$.

Figure 2.11b shows the sweep rate versus $Q_{F}$ plots from the simulation (left panel) and from the experiment (right panel). The simulations reproduce the experimental observation that $Q_{F}$ decreases with increasing sweep rate. Additionally, we find that changing the sweep rate by 20 times only changes the $Q_{F}$ by 4.2 times, which is comparable to the experimental observation that the $Q_{F}$ varies 2.5 times while the sweep rate changes by $\sim 20$ times [9].

The NDR peak from the MC simulations leads to a voltage range of $\sim 0.15 \mathrm{~V}$ while the experimental NDR range is $\sim 0.25 \mathrm{~V}$. One issue is the time scale. Our MC sweep frequency was $\sim 10^{-7} \mathrm{~V} / \mathrm{MCS}$ whereas experimental sweep frequency is $\sim 10^{-1} \mathrm{~V} / \mathrm{s}$. To convert from MCS to second, we compared the initial nucleation rate expressed in MCS to that expected from the transition state theory using the predicted barrier in $\mathrm{kcal} / \mathrm{mol}$. The result is $1 \mathrm{MCS}$ equals $\sim 10^{-13} \mathrm{~s}$ (see Appendix A.), indicating the theoretical sweep frequency $\sim 10^{6}$ times the experimental sweep frequency. This faster sweep should decrease the NDR range. In addition, the small size of our periodic cell, $1620 \mathrm{~nm}^{2}$, compared to the experiment of $O\left(\mathrm{~cm}^{2}\right)$ would also tend to decrease the NDR range.

A limitation in our MC simulations is that we idealized the degrees of freedom for the molecules into two states, which consider as the perfectly crystallized phases where an infinite AN-OPEs are connected through the HB network. In order to investigate the effect of molecular fluctuations, we performed a series of MD simulations with a $(10 \times 10)$ unit cell $(100$ independent AN-OPE molecules). The MD simulations account for two aspects missing from the MC simulations:

1. The AN-OPE is allowed to have a distribution of conformations, accounting for the much lower current for $\chi$ near $90^{\circ}$ while losing the HB network at high fields. This distribution in $\chi$ leads 
to decreased current at a given voltage, leading to a lower slope in the higher-field due to the larger $\Delta \Phi_{B}$.

2. The AN-OPE forms a partially disordered phase with some HB along [10 $\overline{1} 0]$ in the low field (Appendix B). The loss of the HB network at high field decreases the hysteresis during the backward sweep since the loss of HB in the high field decreases the T-to-P transition barrier.

In addition, we found that once a disordered area is developed, it does not easily recover the $\mathrm{P}$ phase; hence, the development of a disordered part on the SAM yields less dramatic changes in conductivity. The experimental observation of slight current decrease with successive sweeps may result from the expansion of the disordered area during the sweep cycles.

In order to validate the suggested mechanism for NDR for AN-OPE, we investigated the possibility of NDR from the system N-OPE which contains no $\mathrm{NH}_{2}$ group and the bare B-OPE containing no functional group. We found that the N-OPE system shows NDR behavior very similar to ANOPE, in agreement with experiment [37]. However, we found no NDR behavior for B-OPE, also in agreement with experiment (Appendix C).

\subsection{Conclusions}

Summarizing, we find that a coarse-grained model based on parameters from first principles calculations leads to a mechanism for room temperature hysteretic NDR that is in qualitative agreement with experiments on AN-OPE, N-OPE, and B-OPE. This provide a plausible mechanism for understanding this phenomena which maybe useful in developing new NDR systems.

\subsection{Acknowledgments}

The computational work was initiated with support by the National Science Foundation (NIRT, WAG). The collaboration was supported by the Microelectronics Advanced Research Corporation (MARCO, WAG and RAK) and its Focus Centers on Functional Engineered NanoArchitectonics (FENA). The facilities of the MSC (WAG) were supported by ONR-DURIP, ARO-DURIP and the facilities of the CNBT lab (SSJ) were supported by the start-up from the MSE in Georgia Tech. 


\section{Bibliography}

[1] Esaki, L. Phys. Rev. 1958, 109, 603-604.

[2] Xue, Y. Q.; Datta, S.; Hong, S.; Reifenberger, R.; Henderson, J. I.; Kubiak, C. P. Phys. Rev. $B$ 1999, 59, R7852-R7855.

[3] Tao, N. J. Nat. Nanotechnol. 2006, 1, 173-181.

[4] Chen, J.; Reed, M. A.; Rawlett, A. M.; Tour, J. M. Science 1999, 286, 1550-1552.

[5] Selzer, Y.; Salomon, A.; Ghabboun, J.; Cahen, D. Angew. Chem. Int. Ed. 2002, 41, 827.

[6] Pitters, J. L.; Wolkow, R. A. Nano Lett. 2006, 6, 390-397.

[7] He, J.; Lindsay, S. M. J. Am. Chem. Soc. 2005, 127, 11932-11933.

[8] Salomon, A.; Arad-Yellin, R.; Shanzer, A.; Karton, A.; Cahen, D. J. Am. Chem. Soc. 2004, $126,11648-11657$.

[9] Kiehl, R. A.; Le, J. D.; Candra, P.; Hoye, R. C.; Hoye, T. R. Appl. Phys. Lett. 2006, 88, 172102.

[10] Guisinger, N. P.; Yoder, N. L.; Hersam, M. C. Proc. Nat. Acad. Sci. U.S.A. 2005, 102, 88388843.

[11] Kratochvilova, I.; Kocirik, M.; Zambova, A.; Mbindyo, J.; Mallouk, T. E.; Mayers, T. S. J. Mater. Chem. 2002, 12, 2927-2930.

[12] Rawlett, A. M.; Hopson, T. J.; Nagahara, L. A.; Tsui, R. K.; Ramachandran, G. K.; Lindsay, S. M. Appl. Phys. Lett. 2002, 81, 3043-3045.

[13] Amlani, I.; Rawlett, A. M.; Nagahara, L. A.; Tsui, R. K. Appl. Phys. Lett. 2002, 80, 2761-2763.

[14] Donhauser, Z. J.; Mantooth, B. A.; Kelly, K. F.; Bumm, L. A.; Monnell, J. D.; Stapleton, J. J.; Price, D. W.; Rawlett, A. M.; Allara, D. L.; Tour, J. M.; Weiss, P. S. Science 2001, 292, 2303-2307.

[15] Fan, F. R. F.; Lai, R. Y.; Cornil, J.; Karzazi, Y.; Bredas, J. L.; Cai, L. T.; Cheng, L.; Yao, Y. X.; Price, D. W.; Dirk, S. M.; Tour, J. M.; Bard, A. J. J. Am. Chem. Soc. 2004, 126, 2568-2573. 
[16] Seminario, J. M.; Zacarias, A. G.; Tour, J. M. J. Am. Chem. Soc. 2000, 122, 3015-3020.

[17] Xiao, X. Y.; Nagahara, L. A.; Rawlett, A. M.; Tao, N. J. J. Am. Chem. Soc. 2005, 127, 9235-9240.

[18] Reed, M. A.; Chen, J.; Rawlett, A. M.; Price, D. W.; Tour, J. M. Appl. Phys. Lett. 2001, 78, $3735-3737$.

[19] Chen, J.; Wang, W.; Reed, M. A.; Rawlett, A. M.; Price, D. W.; Tour, J. M. Appl. Phys. Lett. 2000, 77, 1224-1226.

[20] Sollner, T. C. L. G.; Goodhue, W. D.; Tannenwald, P. E.; Parker, C. D.; Peck, D. D. Appl. Phys. Lett. 1983, 43, 588-590.

[21] Tsuchiya, M.; Sakaki, H.; Yoshino, J. Jpn. J. Appl. Phys., Part 2-Letters 1985, 24, L466-L468.

[22] Qiu, X. H.; Nazin, G. V.; Ho, W. Phys. Rev. Lett. 2004, 93, 4.

[23] Akdim, B.; Pachter, R. J. Phys. Chem. C 2008, 112, 3170-3174.

[24] Troisi, A.; Ratner, M. A. Nano Lett. 2004, 4, 591-595.

[25] Emberly, E. G.; Kirczenow, G. Phys. Rev. B 2001, 64, 125318.

[26] Galperin, M.; Ratner, M. A.; Nitzan, A. Nano Lett. 2005, 5, 125-130.

[27] Kresse, G.; Furthmuller, J. Phys. Rev. B 1996, 54, 11169-11186.

[28] Jaguar, V. 6.5 ed.; Schrödinger Inc.: Portland, 2005.

[29] Kim, Y. H.; Jang, S. S.; Jang, Y. H.; Goddard, W. A. Phys. Rev. Lett. 2005, 94, 156801.

[30] Schultz, P., SeqQuest Project; Sandia National Laboratories: Albuquerque, NM, 2003.

[31] Mayo, S. L.; Olafson, B. D.; Goddard, W. A. J. Phys. Chem. 1990, 94, 8897-8909.

[32] Plimpton, S. J. J. Comput. Phys. 1995, 117, 1-19.

[33] Plimpton, S. J.; Pollock, R.; Stevens, M. The Eighth SIAM Conference on Parallel Processing for Scientific Computing Minneapolis; 1997.

[34] Swope, W. C.; Andersen, H. C.; Berens, P. H.; Wilson, K. R. J. Chem. Phys. 1982, 76, 637-649.

[35] Lo, W. S.; Pelcovits, R. A. Phys. Rev. A 1990, 42, 7471-7474.

[36] Stokbro, K.; Taylor, J.; Brandbyge, M.; Ordejón, P.; Ann. N Y. Acad. Sci. 2003, 1006, 212-216.

[37] Candra, P. M.S. Dissertation, University of Minnesota, 2006. 


\title{
Chapter 3
}

\section{Free Energy Barrier for Molecular Motions in Bistable [2/Rotaxane Molecular Electronic Devices}

\author{
Reproduced with permission from Kim, H; Goddard, W. A.; Jang S. S.; Dichtel, W. R.; Heath, J. \\ R.; and Stoddart J. F. J. Phys. Chem. A 2009, 113, 2136. Copyright 2009 American Chemical \\ Society.
}

\subsection{Abstract}

Donor-acceptor binding of the $\pi$-electron-poor cyclophane cyclobis(paraquat-p-phenylene) (CBPQT

${ }^{4+}$ ) with the $\pi$-electron-rich tetrathiafulvalene (TTF) and 1,5-dioxynaphthalene (DNP) stations provides the basis for electrochemically switchable, bistable [2]rotaxanes, which have been incorporated and operated within solid state devices to form ultradense memory circuits $[1,2]$ and nanoelectromechanical systems. The rate of $\mathrm{CBPQT}^{4+}$ shuttling at each oxidation state of the [2]rotaxane dictates critical write-and-retention time parameters within the devices, which can be tuned through chemical synthesis. To validate how well computational chemistry methods can estimate these rates for use in designing new devices, we used molecular dynamics simulations to calculate the free energy barrier for the shuttling of the $\mathrm{CBPQT}^{4+}$ ring between the TTF and the DNP. The approach used here was to calculate the potential of mean force along the switching pathway, from which we calculated free energy barriers. These calculations find a turn-on time after the rotaxane is doubly oxidized of $\sim 10^{-7} \mathrm{~s}$ (suggesting that the much longer experimental turn-on time is determined by the time scale of oxidization). The return barrier from the DNP to the TTF leads to a predicted lifetime of $2.1 \mathrm{~s}$, which is compatible with experiments. 


\subsection{Introduction}

The electrochemically switchable, bistable [2]rotaxanes [1] (Figure 3.1) developed in recent years by Stoddart and co-workers exhibit two distinct co-conformations [3, 4, 5, 6, 7]: the ground-state co-conformation, in which the cyclobis(paraquat- $p$-phenylene) $\left(\mathrm{CBPQT}^{4+}\right)$ encircles the tetrathiafulvalene (TTF) station, and the metastable state co-conformation, in which the $\mathrm{CBPQT}^{4+}$ encircles the 1,5-dioxynaphthalene (DNP) station $[2,8,9,10,11,12,13,14,15]$. The population of the two co-conformations may be shifted away from equilibrium by temporarily oxidizing one or two electrons from the TTF units. This switching process forms the basis of using these compounds as storage elements in molecular electronic devices. Consequently, significant experimental efforts have been made to investigate the switching behavior of molecular switches $[5,6,16,17,18,19,20,21,22,23]$ and molecular machines $[24,25,26,27,28,29]$ in various environments, such as solution $[5,6,30,31,32,33,34,35,36,37]$, polymer electrolyte gels [38], metal surfaces [39, 40, 41] and devices [7, 16, 17]. Important experimental evidence [15] for molecular switching in these devices was the correlation of the kinetics of relaxation from the DNP to the TTF, across each of these environments. However, the rate of this process is also a function of the molecular structure, suggesting that longer storage times, or even nonvolatile memory, might be possible with the appropriate molecular design.

Computation chemistry calculations could provide an effective approach for optimizing the performance of such molecular switches, but such applications require that the accuracy of the theory be validated by comparing to well-documented experimental results. The purpose of this paper is to provide such validation. Previously, we investigated these compounds using a multiscale first principles approach combining quantum mechanics (QM) and atomistic force field (FF) methods $[42,43,44,45,46,47]$. First we considered the molecules as individual species, and then we examined self-assembled monolayers bound to gold surfaces or compressed into Langmuir monolayers at the air-water interface. These studies successfully predicted a number of phenomena that were confirmed later experimentally, including the higher conductivity [47] of the DNP relative to the TTF, and the increased stability of the TTF relative to the DNP (by $2.0 \mathrm{kcal} / \mathrm{mol}$ from QM, 2.3 $\mathrm{kcal} / \mathrm{mol}$ from the $\mathrm{FF}$, and $1.4-1.6 \mathrm{kcal} / \mathrm{mol}$ from experiment) [15, 43]. In addition, on the basis of the predicted footprint of the $115 \AA^{2} /$ molecule for the self-assembled structure, we predicted that the surface tension of the TTF is $32 \%$ lower than that of the DNP, an observation that was confirmed in subsequent experiements $[43,44]$.

In this study, we evaluated the free energy profile of the shuttling motion of the $\mathrm{CBPQT}^{4+}$ ring between the TTF and the DNP stations to determine how the nature of the rotaxane affects the switching and relaxation rates. These rates have been determined experimentally in various environments $[15,35,40,48]$, and we now want to understand the structural contributions to these 


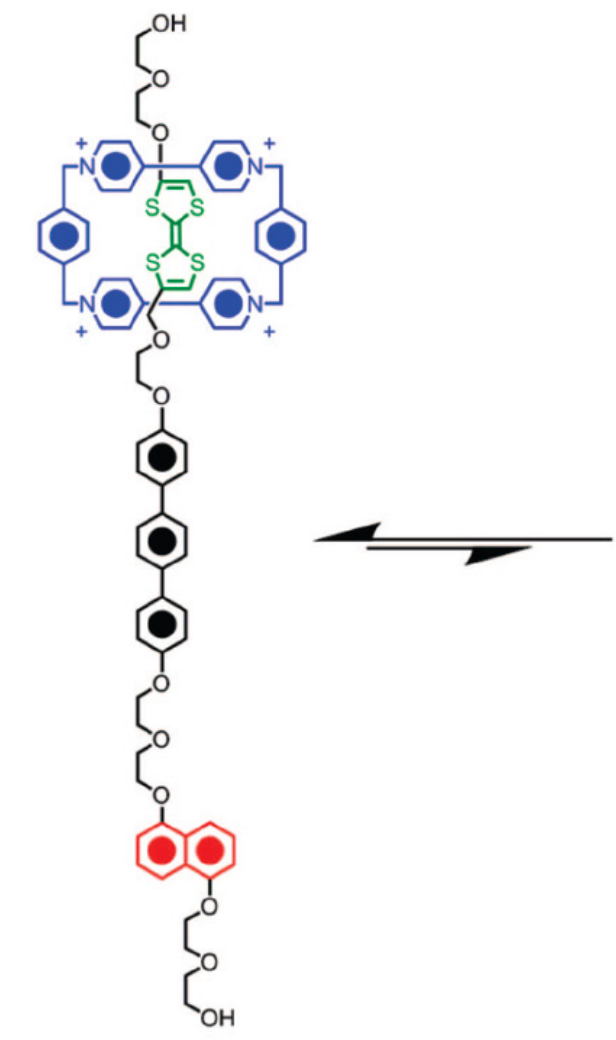

Ground State TTF

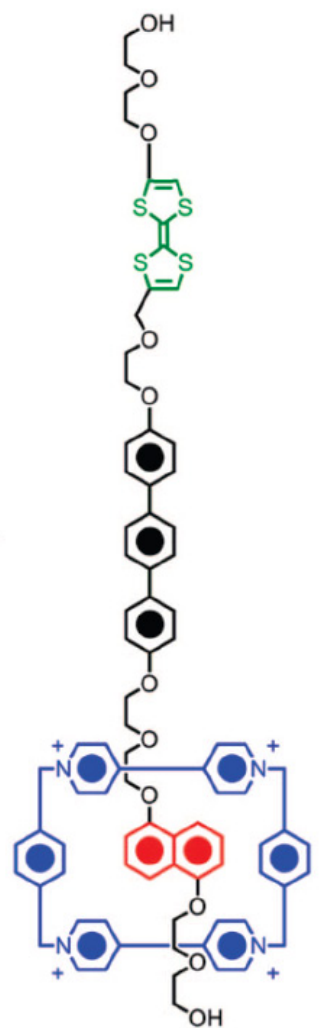

Metastable DNP

Figure 3.1: Structural formula of the two co-conformations of a bistable [2/rotaxane fragment used in this study. 
rates. We seek to find a level for theoretical calculations of these rates that is both accurate and fast so that we can use theory to optimize the structural characteristics to achieve desired rates.

Rather than finding the energy barrier for a minimized reaction path connecting the two states, we used potential of mean force (PMF) to evaluate the change of free energy along the shuttling pathway of the $\mathrm{CBPQT}^{4+}$ ring between the TTF and the DNP so that we can determine the rates at the experimental temperature. We carried out these calculations for three oxidation states of the molecule relevant to the switching and thermally activated relaxation process.

\subsection{Simulation Details}

\subsubsection{Potential of Mean Force from Constrained Molecular Dynamics Simulation}

The experimental time scale for the ring to relax back from the DNP to the TTF is $10^{-1}-10^{3}$ seconds [15, 34, 49], suggesting that simple molecular dynamics (MD) simulation of a few hundred nanoseconds might not be sufficiently ergodic to provide an accurate transition rate. Hence, we adopted the "Blue Moon sampling" technique [50, 51] of constrained MD simulations using holonomic constraints that fix the systems along the reaction coordinate. To determine the free energy barrier, we used the reaction-coordinate $(R)$-dependent potential of mean force (PMF), $F_{\mathrm{rxn}}(R)$ defined as the integration of the mean force $(\mathrm{MF})$ along the reaction coordinate, $-\mathrm{d} F_{\mathrm{rxn}}(R) \mathrm{d} R[52]$,

$$
F_{\text {rxn }}(R)=F_{\text {rxn }}(\infty)+\int_{\infty}^{R} \frac{\mathrm{d} F_{\text {rxn }}\left(R^{\prime}\right)}{\mathrm{d} R^{\prime}} \mathrm{d} R^{\prime}
$$

Here, the MF is a measurable quantity from our simulations. To calculate the MF, we assumed that the $\mathrm{CBPQT}^{4+}$ ring moves between the TTF and the DNP along the backbone of the rotaxane (Figure 3.2a), which we assume to be in an extended conformation but with the minimized structure. This extended conformation should provide the fastest shuttling motion of the $\mathrm{CBPQT}^{4+}$ ring, being governed mainly by its interaction with the backbone. This MF does not account for the presence of folded chain conformations, so that the PMF may lack some contributions from conformational entropy.

First, we prepared the extended rotaxane backbone without the $\mathrm{CBPQT}^{4+}$ ring using quantum mechanical geometry optimization at the level of B3LYP/6-31G* (Figure 3.2a). Then, we added and optimized the $\mathrm{CBPQT}^{4+}$ ring at various fixed points on the fixed extended backbone (Figure 3.2b) using quantum mechanics. Thus, the atomic partial charges of all atoms are allowed to readjust, depending on the relative position of the charge acceptor $\left(\mathrm{CBPQT}^{4+}\right)$ with respect to the charge donor (TTF and DNP).

To obtain the change of the PMF during the shuttling process, we first evaluated the MF as a 


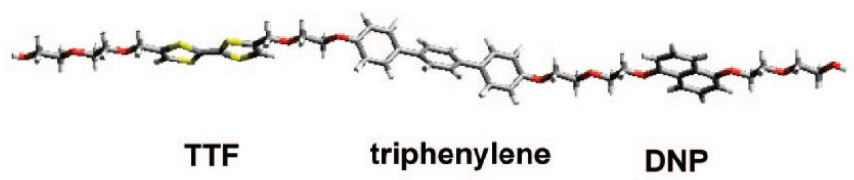

(a)

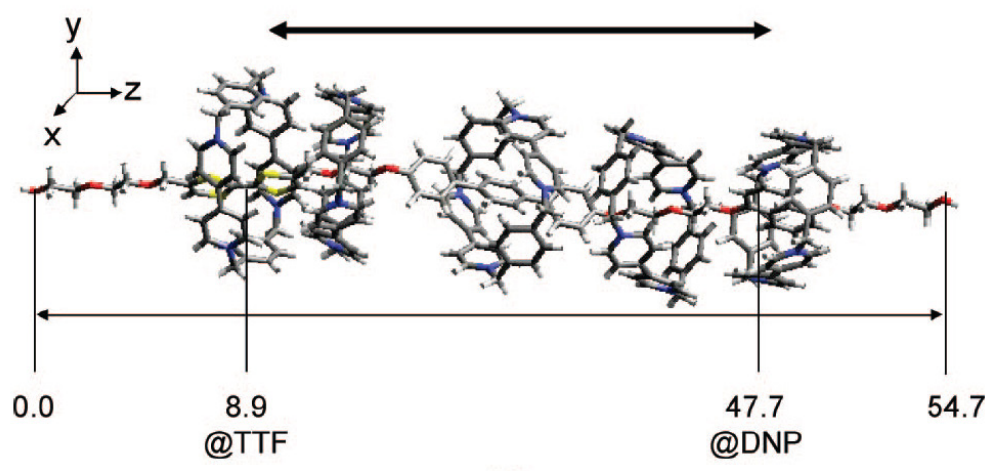

(b)

Figure 3.2: (a) Backbone of the rotaxane molecule simulated in this study. (b) $\mathrm{CBPQT}^{4+}$ ring positions along the backbone (unit: $\AA$ ).

function of the position of the $\mathrm{CBPQT}^{4+}$ ring. Because the length of the backbone is $54.7 \AA$ and the distance between the TTF and the DNP is $36.9 \AA$, we chose to sample the dynamics for nine independent samples, each of which has the $z$-coordinate (along the backbone) of the center of mass $(\mathrm{COM})$ of the $\mathrm{CBPQT}^{4+}$ ring at a different position along the extended backbone, as schematically presented in Figure 3.3. Using quantum mechanics, the geometry and atomic charges were obtained from each of these nine cases.

After preparing these nine initial structures, we prepared two more structures beyond each station of the TTF and the DNP with identical charges to the CBPQT ${ }^{4+}$ @TTF case and the $\mathrm{CBPQT}^{4+} @ \mathrm{DNP}$ case, respectively. In addition, we constructed another ten structures in which the position and charges of the $\mathrm{CBPQT}^{4+}$ ring were calculated by arithmetically averaging the coordinates and charges of two consecutive structures in the eleven structures. Thus, a total of 21 structures were prepared for simulations.

Then, to simulate both the turning on and turning off the rotaxane switch, we investigated the effect of oxidation of rotaxane molecule on the free energy profile, for three different oxidation states: the neutral state, the +1 oxidation state, and the +2 oxidation state.

The QM calculations of the charges for the nine different structures were repeated for each of the three oxidation states: $0,+1$, and +2 . The atomic partial charge distributions are tabulated in the Appendix E (Tables E.1, E.2, and E.3).

All quantum mechanical computations in this study were performed using Jaguar [53]. 


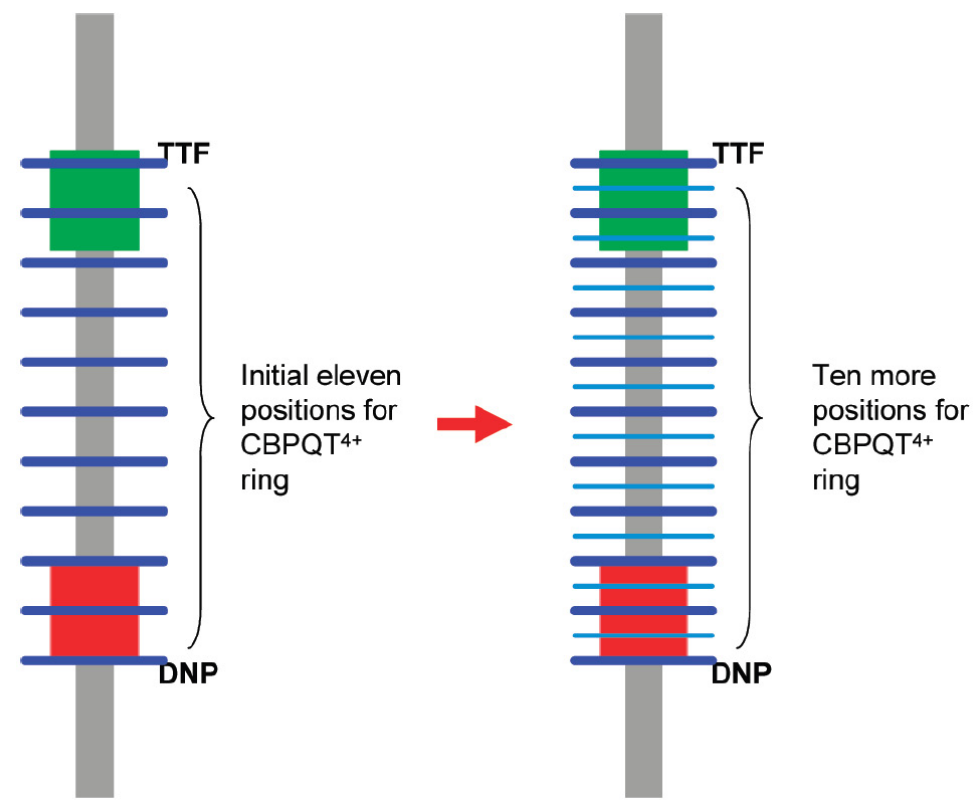

Figure 3.3: Charges for the initial nine structures obtained from QM with Mulliken analysis. In addition, we included two more structures beyond each station of the TTF and the DNP, using charges identical to those for the equilibrium $\mathrm{CBPQT}^{4+} @ \mathrm{TTF}$ and $\mathrm{CBPQT}^{4+} @ \mathrm{DNP}$ cases, respectively. Ten more structures were generated on the basis of these eleven structures. The position of the $\mathrm{CBPQT}^{4+}$ ring for each additional structure was obtained using the arithmetic average of the two adjacent cases from the eleven structures. The charges were also averaged.

\subsubsection{Constrained Molecular Dynamics Simulation}

Next, we carried out a constrained NVT MD simulation at $300 \mathrm{~K}$ for 500 ps to equilibrate each system. This MD was then continued for an additional $3 \mathrm{~ns}$ at $300 \mathrm{~K}$ (constrained NVT MD) to compute the MF. The constraint was introduced using Gauss' principle of least constraints [54] to fix only the $z$-component of the center of mass $(\mathrm{COM})$ of the $\mathrm{CBPQT}^{4+}$ ring parallel to the molecular axis direction ( $z$-axis direction as in Figure $3.2 \mathrm{~b}$ ). To ensure that our constrained dynamics produces the correct equilibrium averages without bias due to ensemble sampling, we used Fixman's theorem [55] to evaluate the metric effect originating from the holonomic constraints. We determined that the metric effect only adds a constant scalar value to the absolute free energy values, which has no influence on the relative energetics. (Details are in the Appendix F.)

We also fixed the position of the last oxygen atom at each end of the backbone to retain the extended conformation. This restricts the conformational flexibility of the system, which suppresses conformational entropic contributions to the free energy. The mean force was sampled from such constrained MD simulations. 


\subsubsection{Force Field and MD Parameters}

We used the generic DREIDING force field [56], which was found to lead to accurate results in our previous studies on rotaxane systems [43, 44, 45]. It was also successful in our studies on various other molecular systems, such as the hydrated polymer electrolyte membranes [57, 58, 59] and the surfactant-mediated air-water interface [60, 61].

The force field has the form

$$
E_{\text {total }}=E_{\mathrm{vdW}}+E_{\mathrm{Q}}+E_{\text {bond }}+E_{\text {angle }}+E_{\text {torsion }}+E_{\text {inversion }}
$$

where $E_{\text {total }}, E_{\mathrm{vdW}}, E_{\mathrm{Q}}, E_{\mathrm{bond}}, E_{\text {angle }}, E_{\text {torsion }}$, and $E_{\text {inversion }}$ are the total energies, the van der Waals, electrostatic, bond stretching, angle bending, torsion, and inversion energy components, respectively, and the force field parameters are described in the original papers [56]. The atomic charges were obtained from a QM Mulliken population analysis as indicated above.

All MD simulations were performed using LAMMPS (large-scale atomic/molecular massively parallel simulator) MD code from Plimpton at Sandia $[62,63]$. The equations of motion were integrated using the velocity-Verlet algorithm [64], with a time step of $0.01 \mathrm{fs}$. This unusually small time step was to ensure high quality sampling of phase space by avoiding abrupt changes in atomic positions.

The temperature was kept constant during the MD using the Berendsen thermostat with temperature damping time of $0.01 \mathrm{fs}$. To demonstrate that our MD leads to a proper canonical ensemble, the probability distribution function $(\mathrm{PDF})$ of kinetic energy $\mathrm{KE}\left(=m v^{2} / 2\right)$ is shown in Figure 3.4. The PDF is quite close to the Maxwell-Boltzmann distribution of energy at $T=300 \mathrm{~K}$, indicating that the simulation describes a proper canonical ensemble. Furthermore, the PDF for each component of velocity is the same and the system obeys the equipartition theorem (Figure 3.5).

Figure 3.6 shows the typical behavior of the MF as a function of simulation time for two representative systems: one is the ground state, CBPQT ${ }^{4+} @ \mathrm{TTF}$, green color, denoted as TTF and the other is the metastable state, the $\mathrm{CBPQT}^{4+}$ ring on the DNP, red color, denoted as DNP. This shows that the mean force was well equilibrated for both cases.

The weakness of this blue moon sampling method is that the error in each MF measurement is integrated to obtain the PMF profile along the reaction coordinate. From block averages, we estimate the uncertainty of the MF values to be $0.04 \mathrm{kcal} / \mathrm{mol} / \AA$ for $\mathrm{CBPQT}^{4+} @ \mathrm{TTF}$ and 0.22 $\mathrm{kcal} / \mathrm{mol} / \AA$ for $\mathrm{CBPQT}^{4+} @ \mathrm{DNP}$. Assuming that these errors are random and that the average value is $0.13 \mathrm{kcal} / \mathrm{mol} / \AA$, we estimate that the error of the free energy difference between two stations is $38.8 \times 0.13 /(20)^{1 / 2}=1.13 \mathrm{kcal} / \mathrm{mol}$ from integrating over the $38.8 \AA$ distance. Similarly the error of the barrier from the DNP toward the TTF is $25.5 \times 0.13 /(14)^{1 / 2}=0.89 \mathrm{kcal} / \mathrm{mol}$ from the integration over the $25.5 \AA$ distance. Hence, the small errors in the MF values can lead to 


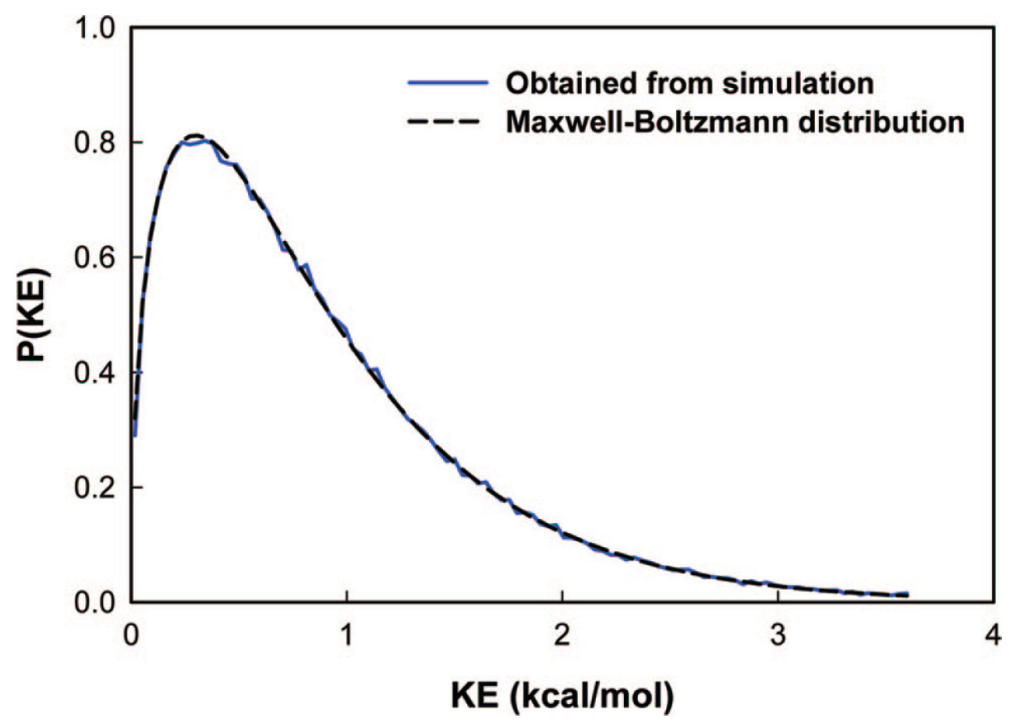

Figure 3.4: Probability density function of kinetic energy KE $\left(=m v^{2} / 2\right)$ is from the MD simulation of the $\mathrm{CBPQT}^{4+}$ ring@TTF (blue line) at $300 \mathrm{~K}$. Here the time step was 0.01 fs and the total simulation time was $3 \mathrm{~ns}$ after $500 \mathrm{ps}$ of equilibration. The black dashed line compares with the Maxwell-Boltzmann distribution of the energy, $2\left(\mathrm{KE} / \pi\left(k_{B} T\right)^{3}\right)^{1 / 2} \exp \left(-\mathrm{KE} / k_{B} T\right)$, for $T=300$ $\mathrm{K}$.

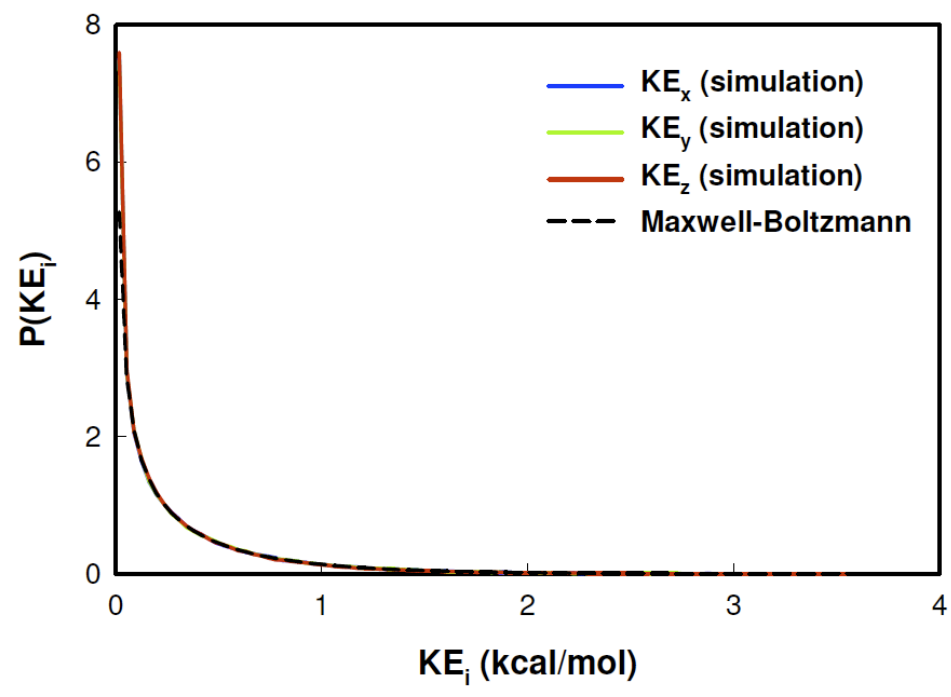

Figure 3.5: Probability density function of $\mathrm{KE}_{x}\left(=m v_{x}^{2} / 2\right.$; blue line $), \mathrm{KE}_{y}\left(=m v_{y}^{2} / 2\right.$; green line), and $\mathrm{KE}_{z}\left(=m v_{z}^{2} / 2\right.$; red line) are cmputed from the MD simulation of the $\mathrm{CBPQT}^{4+}$ ring@TTF (blue line), which are identical to each other. These are compared with the Maxwell-Boltzmann distribution of $\mathrm{KE}_{i}, \sqrt{1 /\left(\pi \mathrm{KE}_{i} k_{B} T\right)} \exp \left(-\mathrm{KE}_{i} / k_{B} T\right)$ at $300 \mathrm{~K} .(i \in\{x, y, z\}$; black dashed line $)$ 

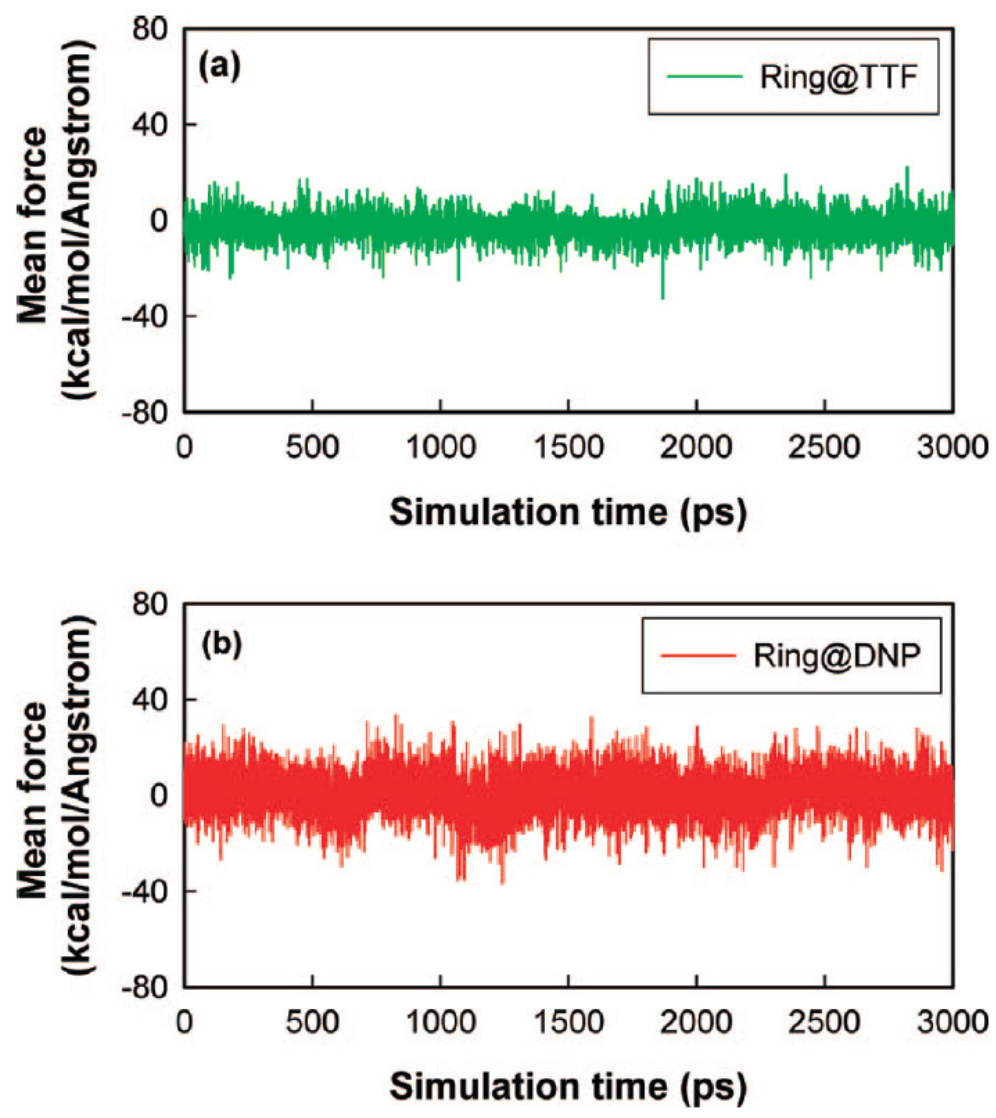

Figure 3.6: Change of mean force as a function of simulation time. In each case this follows $500 \mathrm{ps}$ of equilibration time. This plot shows two representative cases: the $\mathrm{CBPQT}^{4+}$ ring@TTF (TTF) and the $\mathrm{CBPQT}^{4+}$ ring@DNP (DNP).

substantial errors in the PMF value. However, previous studies that carefully compare various PMF calculation methods show that constraint-biased sampling to determine mean forces is one of the best methods to obtain reasonable PMF values, even though, statistically, they contain large error bars [52].

\subsection{Results and Discussion}

\subsubsection{Charge Scheme: Adiabatic Approximation}

We expected that no set of fixed charges scheme would be adequate enough to describe the electrostatic interactions as the highly charged ring is moved along the backbone. Thus, as described in Section 3.3.1, we obtained atomic charges from independent QM at each position as the ring is moved along the backbone.

This assumption of adiabatically adjusted charges assumes that charge re-distribution is much faster than the time for the ring to travel along the backbone. To test the effect of these charge 


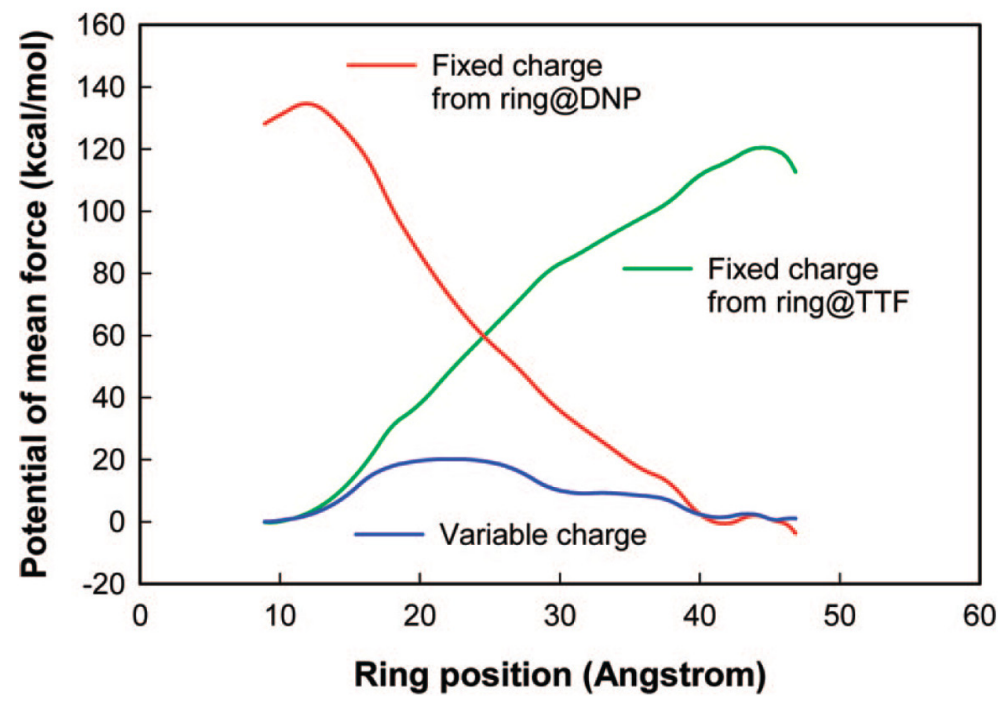

Figure 3.7: Change of potential of mean force as a function of ring position along the backbone. The blue curve allows the charge to change adiabatically as the ring moves along the dumbbell, which is the reliable result. The other two curves show the error obtained when the charges are fixed: the green curve uses fixed charges from the ring@TTF; the red curve uses a fixed charge from the ring@DNP.

re-adjustments on the PMF, Figure 3.7 shows the PMF based on three different charge schemes for the neutral rotaxane system: the green curve was obtained using the fixed charges from the ring@TTF, the red curve was obtained using the fixed charges from the ring@DNP, and the blue curve was obtained using adiabatic charges.

Clearly, the green and red curves are biased to have a minimum PMF at the position for which the charge was calculated, leading to very bad estimates of the barrier. In contrast, the energy barrier between the TTF and the DNP sites, based on the adiabatic charges, is consistent with experimental observations. Thus, we used the adiabatic charges for all oxidation states from the neutral state to the +2 state.

\subsubsection{Free Energy Profiles from PMF Calculations}

Sampling the MFs from the constrained MD simulations (Figure 3.8a) and integrating them along the ring position, we calculated the profile of the PMF for the shuttling motion of the $\mathrm{CBPQT}^{4+}$ ring (Figure $3.8 \mathrm{~b})$. We found that each oxidation state (neutral state $(0)$, oxidized states $(+1$ and $+2)$ ), leads to significantly different profiles.

\subsubsection{1 $\Delta G_{\mathrm{T} 2 \mathrm{D}}$}

We calculated that the most stable complex for the neutral state (black) is CBPQT ${ }^{4+} @$ TTF (ring at $8.9 \AA$ ) whereas the $\mathrm{CBPQT}^{4+} @ \mathrm{DNP}$ state (ring at $47.7 \AA$ ) is less stable than the TTF by 

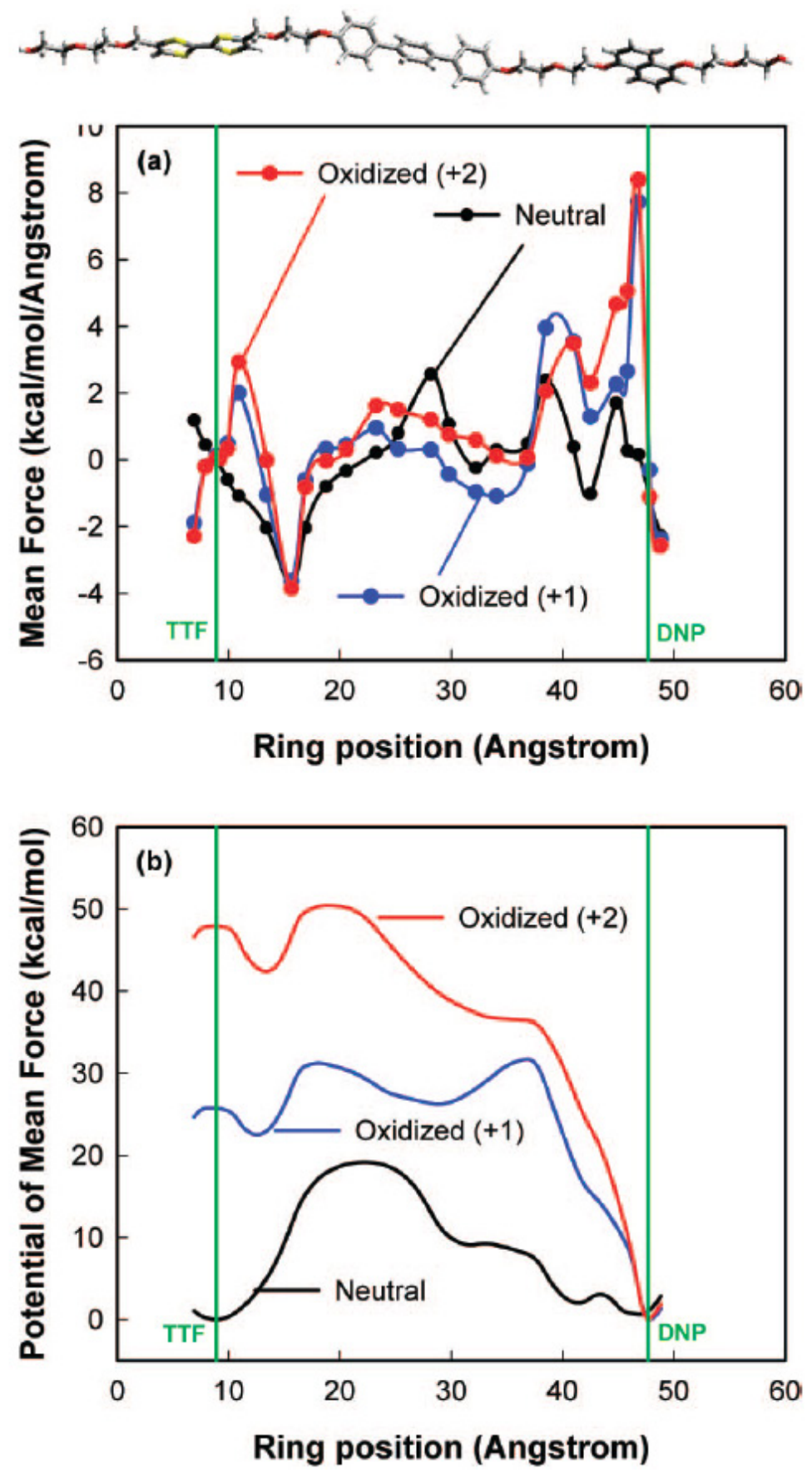

Figure 3.8: (a) Change of the mean force as a function of ring position along the backbone. (b) Change of the potential of mean force as a function of ring position along the backbone. The green

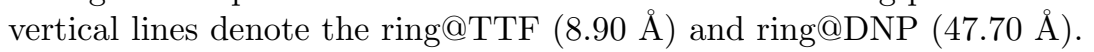


$\Delta G_{\mathrm{T} 2 \mathrm{D}}=1.0 \mathrm{kcal} / \mathrm{mol}$. This agrees with various experiments, which lead to $\Delta G_{\mathrm{T} 2 \mathrm{D}}=1.4-1.6$ $\mathrm{kcal} / \mathrm{mol}[15,65,66,67,68]$ on the basis of the difference in the binding free energies of the individual components of the rotaxane in the MeCN solvent. In addition, this calculation agrees with our previous computations from QM $\left(\Delta G_{\mathrm{T} 2 \mathrm{D}}=2.0 \mathrm{kcal} / \mathrm{mol}\right)[43]$ and Hessian-based FF calculations $\left(\Delta G_{\mathrm{T} 2 \mathrm{D}}=2.3 \mathrm{kcal} / \mathrm{mol}\right)[43]$. We emphasize here that all previous experimental and theoretical studies studied $\Delta G_{\mathrm{T} 2 \mathrm{D}}$ by comparing the binding free energy of the TTF derivatives with the $\mathrm{CBPQT}^{4+}$ ring and the binding free energy of the DNP derivatives with the $\mathrm{CBPQT}^{4+}$ ring. Thus, our current calculation is the first direct measurement of the $\Delta G_{\mathrm{T} 2 \mathrm{D}}$ on a complete rotaxane.

\subsubsection{2 $\Delta G_{\mathrm{T} 2 \mathrm{D}}^{\ddagger}$ and $\Delta G_{\mathrm{D} 2 \mathrm{~T}}^{\ddagger}$ for Neutral Case}

We calculate that the free energy barrier is $\Delta G_{\mathrm{T} 2 \mathrm{D}}^{\ddagger}=19.03 \mathrm{kcal} / \mathrm{mol}$ from the TTF toward the $\mathrm{DNP}$, and $\Delta G_{\mathrm{D} 2 \mathrm{~T}}^{\ddagger}=18.03 \mathrm{kcal} / \mathrm{mol}$ in the opposite direction. The relaxation barrier in the neutral state was measured for a similar bistable [2]rotaxane in which the triphenylene spacer was missing, leading to [15] (see Table 3.1)

- $\Delta G_{\mathrm{D} 2 \mathrm{~T}}^{\ddagger}=16.2 \mathrm{kcal} / \mathrm{mol}(\tau \sim 0.095 \mathrm{~s})$ in MeCN solvent,

- $\Delta G_{\mathrm{D} 2 \mathrm{~T}}^{\ddagger}=18.1 \mathrm{kcal} / \mathrm{mol}(\tau \sim 2.4 \mathrm{~s})$ in a MeCN/polymethylmethacrylate/propylene carbonate/ $\mathrm{LiClO}_{4}$ polymer matrix containing weight ratios of 70:7:20:3, and

- $\Delta G_{\mathrm{D} 2 \mathrm{~T}}^{\ddagger}=22.21 \mathrm{kcal} / \mathrm{mol}\left(\tau \sim 2.5 \times 10^{3} \mathrm{~s}\right)$ in the molecular switch tunnel junction.

In addition, the devices fabricated with this derivative containing the triphenylene spacer exhibit a relaxation half-life of $\tau \sim 90 \min [2]\left(\Delta G_{\mathrm{D} 2 \mathrm{~T}}^{\ddagger}=22.66 \mathrm{kcal} / \mathrm{mol}\right)$.

In addition, our free energy barrier is quite comparable to the barriers to circumrotation of [2]catenanes. Leigh and co-workers used NMR to determine $\Delta G^{\ddagger}$ of interlocked catenane molecules as $11-20 \mathrm{kcal} / \mathrm{mol}$ for various solvents and calculated the free energy barrier as $10-20 \mathrm{kcal} / \mathrm{mol}$ using force-field based Hessians [69, 70, 71].

Although our simulations were performed in the gas phase, the $\Delta G_{\mathrm{D} 2 \mathrm{~T}}^{\ddagger}$ of $18 \mathrm{kcal} / \mathrm{mol}$ agrees well with the experimental barriers $(17-22 \mathrm{kcal} / \mathrm{mol})[15,34,49]$ for a variety of environments. This suggests that the energy barrier does not depend strongly on environment.

We did not include the counterions in this study because preliminary calculations showed that the charges would sometimes change in erratic ways due to the floppy energy landscape for the countercharges. Indeed, the good agreement with experiment for the barriers suggests that the instantaneous changes in the potential due to counterions can be neglected. 


\subsubsection{3 $\Delta G_{\mathrm{T} 2 \mathrm{D}}^{\ddagger}$ and $\Delta G_{\mathrm{D} 2 \mathrm{~T}}^{\ddagger}$ for Oxidized Cases}

Although the neutral state prefers to have the $\mathrm{CBPQT}^{4+}$ ring at the TTF, we find that the +1 and +2 oxidized states lead to a completely different energy profile (Figure 3.8b). In both cases, the DNP becomes the global minimum with the TTF destabilized by $\Delta G=25.75 \mathrm{kcal} / \mathrm{mol}$ for the +1 oxidation state and $\Delta G=47.78 \mathrm{kcal} / \mathrm{mol}$ for the +2 oxidation state.

Starting with the ring at the TTF site and oxidizing, we find that the ring moves first by $\sim 5 \AA$ to a local minimum on the ethylene oxide linker (with an energy decrease by $\Delta G=3.25 \mathrm{kcal} / \mathrm{mol}$ for the +1 and $\Delta G=5.49 \mathrm{kcal} / \mathrm{mol}$ for the +2$)$. Then, it has a free energy barrier of $\Delta G=8.70$ $\mathrm{kcal} / \mathrm{mol}(+1 \mathrm{state})$ or $8.02 \mathrm{kcal} / \mathrm{mol}(+2$ state $)$ to continue past the triphenylene spacer and toward the DNP for oxidation states.

Using the Eyring rate equation $\left[1 / \tau=\left(k_{B} T / h\right) \exp \left(-\Delta G^{\ddagger} / R T\right)\right]$, the time required to overcome this barrier to move onto the DNP is $2.9 \times 10^{-7} \mathrm{~s}$ for the +1 oxidation state and $9.0 \times 10^{-8} \mathrm{~s}$ for the +2 oxidation state. It would be interesting to design an experiment to probe for this predicted barrier. It has been assumed that the huge Coulomb potential of the +4 ring with the +2 TTF would preclude a barrier. The origin of this barrier in the oxidized state is discussed below, which we find arises from the triphenylene spacer. We expect that there would be no barrier without this spacer.

Relative to the final state of the ring at the DNP site, the energy at the ethylene oxide linker (EO) near the TTF site is $22.52 \mathrm{kcal} / \mathrm{mol}$ higher (+1 oxidation), leading to a Boltzmann population of $10^{-17}$. For the +2 oxidation state, the energy is $42.41 \mathrm{kcal} / \mathrm{mol}$ higher, leading to a population of $10^{-32}$. Thus, for oxidation states +1 and +2 , we expect the $\mathrm{CBPQT}^{4+}$ ring to stay on the DNP site until the system is reduced.

Indeed, there is an experimental estimate of this reverse barrier. Using a modified AFM with the ring attached, Brough et al. [72] measured the force exerted on the ring shuttling from the DNP to the TTF in the +2 oxidized system as $145 \mathrm{pN}$. Combining this experimental data with results from molecular mechanics simulations, they estimated the energy barrier to be $65 \mathrm{kcal} / \mathrm{mol}$. This can be compared to our calculated barrier of $50.4 \mathrm{kcal} / \mathrm{mol}$ energy, validating the accuracy of the experiment. The maximum force measured in our simulation during the ring shuttling is $583 \mathrm{pN}$, which is similar to the experimental value of $145 \mathrm{pN}$.

\subsubsection{Effect of Coulombic Energy and van der Waals Energy}

To understand why the PMF profiles are so different between the neutral, +1 , and +2 oxidation states, we calculated the change in the Coulombic interaction energy and the van der Waals (vdW) interaction energy as a function of ring position along the backbone, for these three oxidation states (Figures 3.9 and 3.10). 
Table 3.1: Free Energy Barriers, Rate Constants, and Relaxation Half-Lives from DNP toward TTF $(\mathrm{DNP} \rightarrow \mathrm{TTF})$ at $298 \mathrm{~K}$ (All Simulation Results from this Work)

\begin{tabular}{|c|c|c|c|}
\hline$\Delta G(\mathrm{kcal} / \mathrm{mol})$ & ${ }^{a} k\left(\mathrm{~s}^{-1}\right)$ & ${ }^{a} \tau_{1 / 2}(\mathrm{~s})$ & condition \\
\hline $\begin{array}{c}18.03 \pm 1.5 \\
\text { (simul.) }\end{array}$ & $0.33 \pm 0.83$ & $2.1 \pm 5.4$ & $\begin{array}{l}\text { gas phase } \\
\text { (neutral) }\end{array}$ \\
\hline $\begin{array}{l}16.2 \pm 0.3 \\
(\exp .[34])\end{array}$ & $7.3 \pm 3.7$ & $0.095 \pm 0.048$ & $\begin{array}{c}\left(\mathrm{CH}_{3} \mathrm{CN}\right) \\
\text { (neutral) }\end{array}$ \\
\hline $\begin{array}{c}18.1 \pm 0.2 \\
(\exp .[15,34,71])\end{array}$ & $0.3 \pm 0.10$ & $2.4 \pm 0.082$ & $\begin{array}{c}{ }^{b} \text { polymer matrix } \\
(\text { neutral })\end{array}$ \\
\hline $\begin{array}{c}22.21 \pm 0.04 \\
(\exp .[15,34,71])\end{array}$ & $(2.7 \pm 0.19) \times 10^{-4}$ & $(2.5 \pm 0.18) \times 10^{3}$ & $\begin{array}{l}\text { molecular-switch junction } \\
\text { (neutral) }\end{array}$ \\
\hline $\begin{array}{c}31.22 \\
(\text { simul. })\end{array}$ & $6.3 \times 10^{-11}$ & $1.1 \times 10^{10}$ & $\begin{array}{l}\text { gas phase } \\
\text { (oxidation }+1)\end{array}$ \\
\hline $\begin{array}{c}50.43 \\
(\text { simul. })\end{array}$ & $4.5 \times 10^{-25}$ & $1.5 \times 10^{24}$ & $\begin{array}{l}\text { gas phase } \\
\text { (oxidation }+2 \text { ) }\end{array}$ \\
\hline $\begin{aligned} & 65 \\
&(\text { exp. }+ \text { simul. }[72]) \\
&\end{aligned}$ & - & - & $\begin{array}{c}{ }^{c} \mathrm{SAM} \text { on } \mathrm{SiO}_{2} \text { wafers } \\
(\text { oxidation }+2)\end{array}$ \\
\hline
\end{tabular}

${ }^{a}$ Values are calculated using the Eyring equation, $1 / \tau=\left(k_{B} T / h\right) \exp \left(-\Delta G^{\ddagger} / R T\right)$. ${ }^{b}$ Weight ratio 70:7:20:3 for $\mathrm{CH}_{3} \mathrm{CN} /$ poly(methylmethacrylate)/propylene carbonate/LiClO $4 .{ }^{c}$ The modified AFM tip is attached to the $\mathrm{CBPQT}^{4+}$ ring.

For the neutral state, we find that the Coulombic energy increases by $60 \mathrm{kcal} / \mathrm{mol}$ as the ring moves from the TTF to the triphenylene spacer (barrier) and then drops by $45 \mathrm{kcal} / \mathrm{mol}$ as it moves to the DNP. On the other hand, the vdW energy changes, within a range of $\pm 4 \mathrm{kcal} / \mathrm{mol}$, while the ring travels from the TTF to the DNP.

This indicates that the barrier is dominated by the differential Coulombic interactions with a peak of $443 \mathrm{kcal} / \mathrm{mol}$ at $z=28 \AA$ (over the spacer). We were quite surprised because we expected the barrier to be dominated by vdW repulsions due to the bulky size of the triphenylene. To understand why Coulombic interactions are so important, we plot in Figure 3.11 the total charge on the ring along the pathway, in the neutral case. We see that at the TTF or the DNP positions there is strong delocalization from the ring onto the backbone, but as the ring passes over the triphenylene spacer (at $z=28 \AA$ ), this charge localizes back onto the ring. Thus, we conclude that localization of the ring charge increases the Coulombic repulsion and dominates the free energy barrier. This suggests that the barrier can be modified dramatically by changing the polarity of the spacer.

We also found that as the system is oxidized, the magnitude of Coulombic repulsion increases from $380-445 \mathrm{kcal} / \mathrm{mol}$ for the neutral state, to $520-545 \mathrm{kcal} / \mathrm{mol}$ for the +1 oxidation state, and finally, to $670-700 \mathrm{kcal} / \mathrm{mol}$ for the +2 oxidation state. In contrast, the vdW energy changes from $165-173$ to $165-172$ to $164-171 \mathrm{kcal} / \mathrm{mol}$ as the system is oxidized. This implies that the driving force inducing the mechanical movement of the ring is the increased Coulombic repulsion due to oxidization of the rotaxane. This confirms our view since the beginning of our experiments.

However, the PMF profile (Figure 3.7b) still differs substantially from the Coulombic energy 

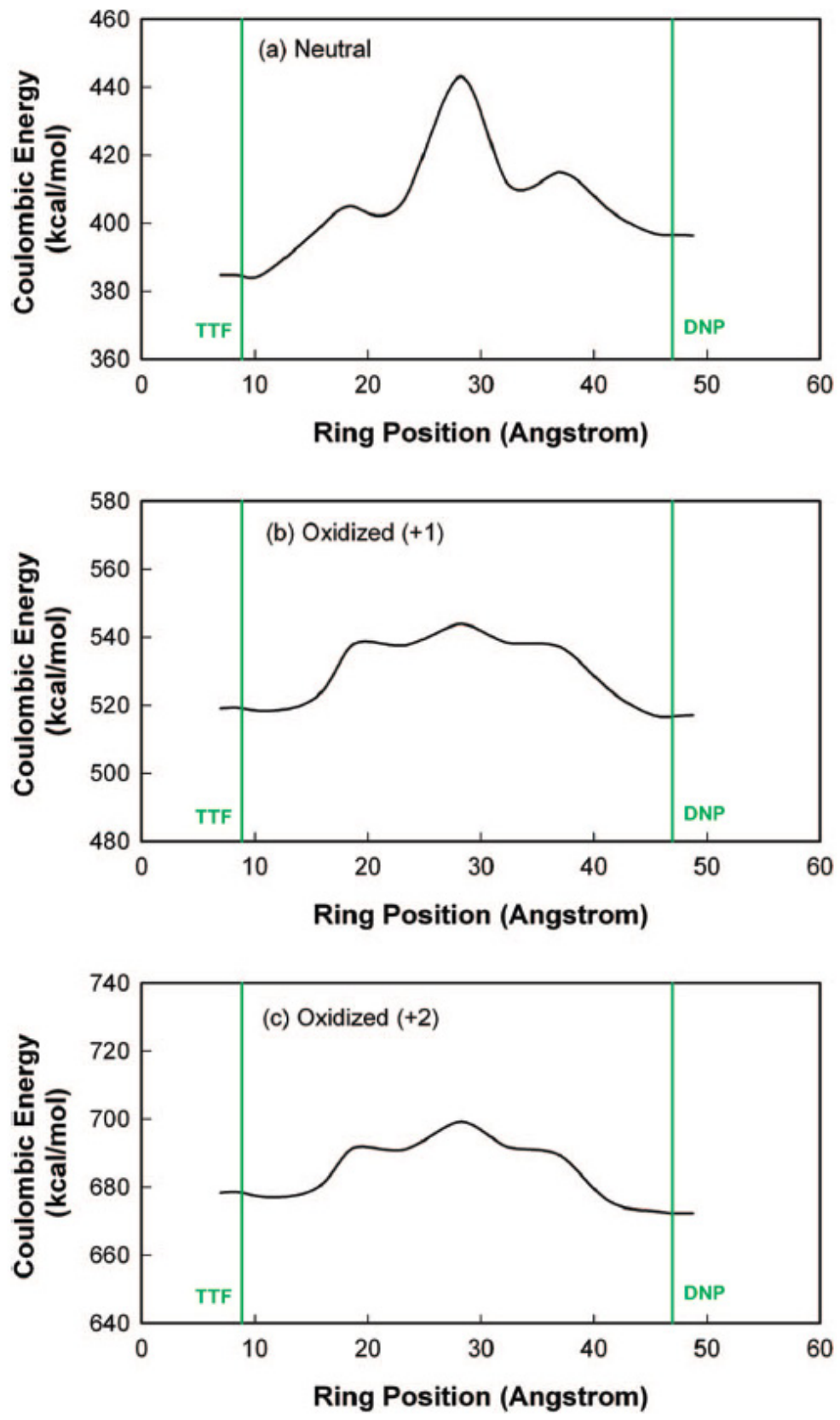

Figure 3.9: Change of Coulombic interaction energy as a function of the ring position: (a) neutral state; (b) oxidation state +1 ; (c) oxidation state +2 . 


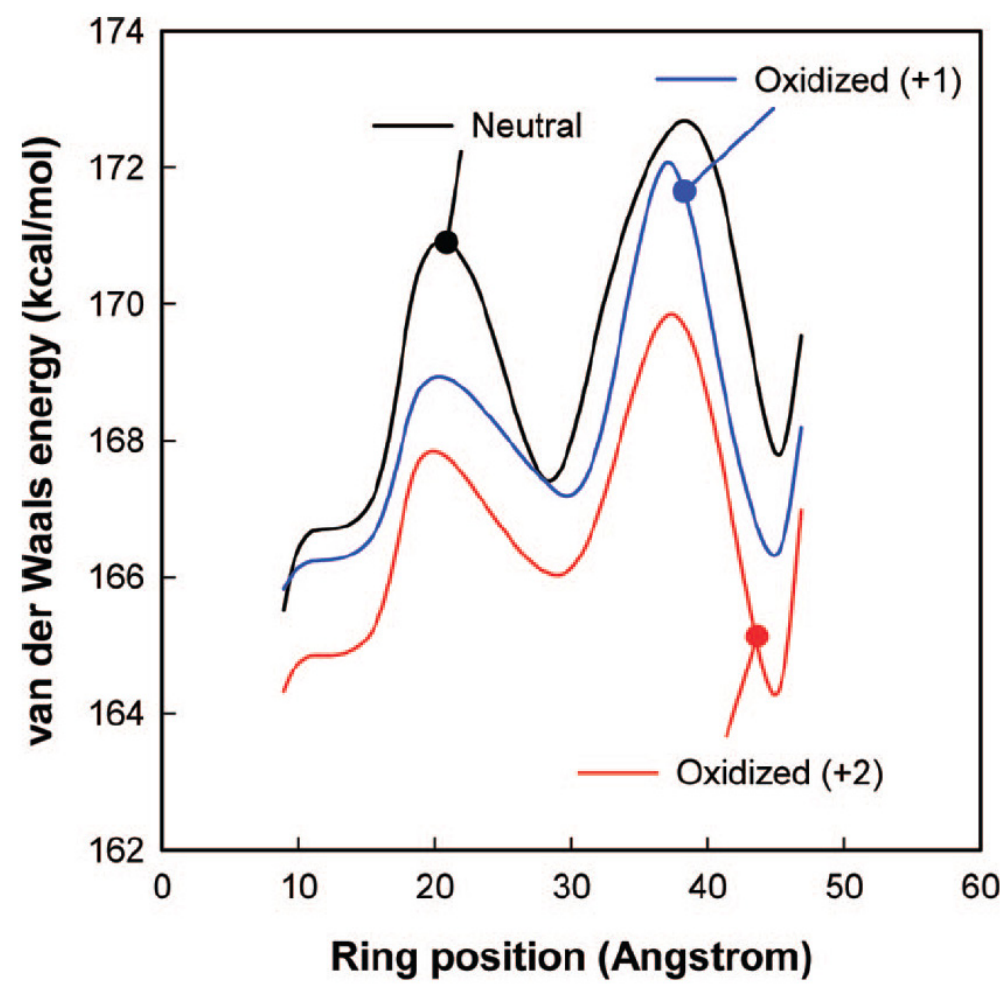

Figure 3.10: Change of van der Waals interaction energy as a function of the ring position: (a) neutral state; (b) oxidation state +1 ; (c) oxidation state +2 .

profile (Figure 3.9). For instance, in the neutral case, the Coulombic energy difference between two stations is $12.03 \mathrm{kcal} / \mathrm{mol}$, which is $\sim 12$ times larger than $\Delta G_{\mathrm{T} 2 \mathrm{D}}=1.0 \mathrm{kcal} / \mathrm{mol}$, and the Coulombic energy barrier for the shuttling from the DNP to the TTF is $46.75 \mathrm{kcal} / \mathrm{mol}$, which is $\sim 2.6$ times larger than $\Delta G_{\mathrm{D} 2 \mathrm{~T}}^{\ddagger}=18.03 \mathrm{kcal} / \mathrm{mol}$. Thus, the key features of the PMF profile are not fully explained in terms of the Coulombic energy alone. Another possible contributor to the free energy is vibrational entropy, which can be investigated directly from the MD simulation trajectory $[73,74]$.

\subsection{Conclusions}

We used constrained MD simulations to calculate the free energy profile at $300 \mathrm{~K}$ for the shuttling of the $\mathrm{CBPQT}^{4+}$ ring between the TTF and the DNP in the rotaxane molecule. This free energy profile was derived by calculating and integrating the MF acting on the ring as it is moved from one position to another position along the backbone. We found that it is particularly important to allow the charges to adjust adiabatically as the ring moves. Indeed, we find that the Coulomb interactions dominate the barriers for these systems.

We found that the free energy barrier from the DNP to the TTF is $18.03 \mathrm{kcal} / \mathrm{mol}$ for the neutral 


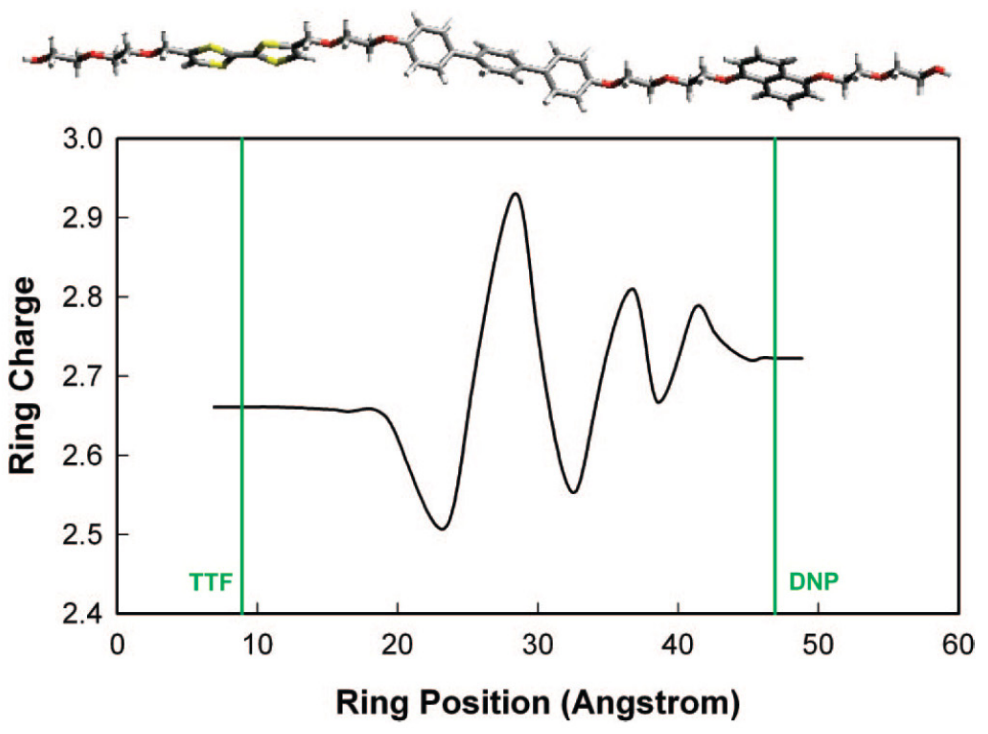

Figure 3.11: Variations in the total charge on the ring as a function of the ring position for the neutral case.

system, which agrees well with experimental values of $17-22 \mathrm{kcal} / \mathrm{mol}$ for various environments. We calculate that the $\Delta G$ between the TTF and the DNP positions is $1.0 \mathrm{kcal} / \mathrm{mol}$, which compares well with experimental results of $1.4-1.6 \mathrm{kcal} / \mathrm{mol}$ obtained from binding energies of separate DNP and TTF systems with the $\mathrm{CBPQT}^{4+}$ ring.

These results validate the accuracy of our computational procedure. Thus, we can now use this validated technique for estimating the switching kinetics for new designs of molecular architectures.

\subsection{Acknowledgments}

The computational work was initiated with support by the National Science Foundation (NIRT, W.A.G.). The collaboration was supported by the Microelectronics Advanced Research Corporation (MARCO; W.A.G. and J.F.S.) and its Focus Centers on Functional Engineered NanoArchitectonics (FENA) and Materials Structures and Devices, the Molectronics Program of the Defense Advanced Research Projects Agency (DARPA; J.F.S. and J.R.H.), the Center for Nanoscale Innovation for Defense (CNID; J.F.S.), and the MARCO Materials Structures and Devices Focus Center (J.R.H.). In addition, the facilities of the MSC (W.A.G.) were supported by ONR-DURIP and ARO-DURIP. 


\section{Bibliography}

[1] Luo, Y.; Collier, C. P.; Jeppesen, J. O.; Nielsen, K. A.; Delonno, E.; Ho, G.; Perkins, J.; Tseng, H. R.; Yamamoto, T.; Stoddart, J. F.; Heath, J. R. ChemPhysChem 2002, 3, 519-525.

[2] Green, J. E.; Choi, J. W.; Boukai, A.; Bunimovich, Y.; Johnston-Halperin, E.; DeIonno, E.; Luo, Y.; Sheriff, B. A.; Xu, K.; Shin, Y. S.; Tseng, H.-R.; Stoddart, J. F.; Heath, J. R. Nature 2007, 445, 414-417.

[3] Anelli, P. L.; Spencer, N.; Stoddart, J. F. J. Am. Chem. Soc. 1991, 113, 5131-5133.

[4] Bissell, R. A.; Cordova, E.; Kaifer, A. E.; Stoddart, J. F. Nature 1994, 369, 133-137.

[5] Asakawa, M.; Ashton, P. R.; Balzani, V.; Credi, A.; Hamers, C.; Mattersteig, G.; Montalti, M.; Shipway, A. N.; Spencer, N.; Stoddart, J. F.; Tolley, M. S.; Venturi, M.; White, A. J. P.; Williams, D. J. Angew. Chem. Int. Ed. 1998, 37, 333-337.

[6] Balzani, V.; Credi, A.; Mattersteig, G.; Matthews, O. A.; Raymo, F. M.; Stoddart, J. F.; Venturi, M.; White, A. J. P.; Williams, D. J. J. Org. Chem. 2000, 65, 1924-1936.

[7] Collier, C. P.; Mattersteig, G.; Wong, E. W.; Luo, Y.; Beverly, K.; Sampaio, J.; Raymo, F. M.; Stoddart, J. F.; Heath, J. R. Science 2000, 289, 1172-1175.

[8] Barboiu, M.; Lehn, J.-M. Proc. Natl. Acad. Sci. U.S.A. 2002, 99, 5201-5206.

[9] Hogg, L.; Leigh, D. A.; Lusby, P. J.; Morelli, A.; Parsons, S.; Wong, J. K. Y. Angew. Chem., Int. Ed. 2004, 43, 1218-1221.

[10] Zheng, X.; Mulcahy, M. E.; Horinek, D.; Galeotti, F.; Magnera, T. F.; Michl, J. J. Am. Chem. Soc. 2004, 126, 4540-4542.

[11] Hawthorne, M. F.; Zink, J. I.; Skelton, J. M.; Bayer, M. J.; Liu, C.; Livshits, E.; Baer, R.; Neuhauser, D. Science 2004, 303, 1849-1851.

[12] de Jong, J. J. D.; Lucas, L. N.; Kellogg, R. M.; van Esch, J. H.; Reringa, B. L. Science 2004, 304, 278-281. 
[13] Turberfield, A. J.; Mitchell, J. C.; Yurke, B.; Mills, A. P.; Blakey, M. I.; Simmel, F. C. Phys. Rev. Lett. 2003, 90, art. no.118102.

[14] Liu, H. Q.; Schmidt, J. J.; Bachand, G. D.; Rizk, S. S.; Looger, L. L.; Hellinga, H. W.; Montemagno, C. D. Nat. Mater. 2002, 1, 173-177.

[15] Choi, J. W.; Flood, A.; Steuerman, D. W.; Nygaard, S.; Braunschweig, A.; Moonen, N.; Laursen, B.; Luo, Y.; DeIonno, E.; Peters, A. J.; Jeppesen, J. O.; Stoddart, J. F.; Heath, J. R. Chem. Eur. J. 2006, 12, 261-279.

[16] Collier, C. P.; Jeppesen, J. O.; Luo, Y.; Perkins, J.; Wong, E. W.; Heath, J. R.; Stoddart, J. F. J. Am. Chem. Soc. 2001, 123, 12632-12641.

[17] Diehl, M. R.; Steuerman, D. W.; Tseng, H. R.; Vignon, S. A.; Star, A.; Celestre, P. C.; Stoddart, J. F.; Heath, J. R. ChemPhysChem 2003, 4, 1335-1339.

[18] Credi, A.; Balzani, V.; Langford, S. J.; Stoddart, J. F. J. Am. Chem. Soc. 1997, 119, 2679-2681.

[19] Collier, C. P.; Wong, E. W.; Belohradsky, M.; Raymo, F. M.; Stoddart, J. F.; Kuekes, P. J.; Williams, R. S.; Heath, J. R. Science 1999, 285, 391-394.

[20] Elizarov, A. M.; Chiu, S. H.; Stoddart, J. F. J. Org. Chem. 2002, 67, 9175-9181.

[21] Carroll, R. L.; Gorman, C. B. Angew. Chem. Int. Ed. 2002, 41, 4379-4400.

[22] Yu, H. B.; Luo, Y.; Beverly, K.; Stoddart, J. F.; Tseng, H. R.; Heath, J. R. Angew. Chem. Int. Ed. 2003, 42, 5706-5711.

[23] Heath, J. R.; Ratner, M. A. Phys. Today 2003, 56, 43-49.

[24] Balzani, V.; Gomez-Lopez, M.; Stoddart, J. F. Acc. Chem. Res. 1998, 31, 405-414.

[25] Balzani, V.; Credi, A.; Raymo, F. M.; Stoddart, J. F. Angew. Chem. Int. Ed. 2000, 39, 33493391.

[26] Chia, S. Y.; Cao, J. G.; Stoddart, J. F.; Zink, J. I. Angew. Chem. Int. Ed. 2001, 40, 2447-2451.

[27] Belohradsky, M.; Elizarov, A. M.; Stoddart, J. F. Collect. Czech. Chem. Commun. 2002, 67, 1719-1728.

[28] Hernandez, R.; Tseng, H. R.; Wong, J. W.; Stoddart, J. F.; Zink, J. I. J. Am. Chem. Soc. 2004, 126, 3370-3371.

[29] Badjic, J. D.; Balzani, V.; Credi, A.; Silvi, S.; Stoddart, J. F. Science 2004, 303, 1845-1849.

[30] Tseng, H. R.; Vignon, S. A.; Stoddart, J. F. Angew. Chem., Int. Ed. 2003, 42, 1491-1495. 
[31] Jeppesen, J. O.; Perkins, J.; Becher, J.; Stoddart, J. F. Angew. Chem. Int. Ed. 2001, 40, 1216-1221.

[32] Jeppesen, J. O.; Nielsen, K. A.; Perkins, J.; Vignon, S. A.; Di Fabio, A.; Ballardini, R.; Gandolfi, M. T.; Venturi, M.; Balzani, V.; Becher, J.; Stoddart, J. F. Chem. Eur. J. 2003, 9, 2982-3007.

[33] Yamamoto, T.; Tseng, H. R.; Stoddart, J. F.; Balzani, V.; Credi, A.; Marchioni, F.; Venturi, M. Collect. Czech. Chem. Commun. 2003, 68, 1488-1514.

[34] Tseng, H. R.; Vignon, S. A.; Celestre, P. C.; Perkins, J.; Jeppesen, J. O.; Di Fabio, A.; Ballardini, R.; Gandolfi, M. T.; Venturi, M.; Balzani, V.; Stoddart, J. F. Chem. Eur. J. 2004, $10,155-172$.

[35] Kang, S. S.; Vignon, S. A.; Tseng, H. R.; Stoddart, J. F. Chem. Eur. J. 2004, 10, 2555-2564.

[36] Livoreil, A.; Dietrichbuchecker, C. O.; Sauvage, J. P. J. Am. Chem. Soc. 1994, 116, 9399-9400.

[37] Flood, A. H.; Peters, A. J.; Vignon, S. A.; Steuerman, D. W.; Tseng, H.-R.; Kang, S.; Heath, J. R.; Stoddart, J. F. Chem. Eur. J. 2004, 24, 6558-6561.

[38] Steuerman, D. W.; Tseng, H.-R.; Peters, A. J.; Flood, A. H.; Jeppesen, J. O.; Nielsen, K. A.; Stoddart, J. F.; Heath, J. R. Angew. Chem. Int. Ed. 2004, 43, 6486-6491.

[39] Lee, I. C.; Frank, C. W.; Yamamoto, T.; Tseng, H.-R.; Flood, A. H.; Stoddart, J. F.; Jeppesen, J. O. Langmuir 2004, 20, 5809-5828.

[40] Tseng, H. R.; Wu, D. M.; Fang, N. X. L.; Zhang, X.; Stoddart, J. F. ChemPhysChem 2004, 5, 111-116.

[41] Raehm, L.; Kern, J. M.; Sauvage, J. P.; Hamann, C.; Palacin, S.; Bourgoin, J. P. Chem. Eur. J. 2002, 8, 2153-2162.

[42] Jang, Y. H.; Kim, Y. H.; Jang, S. S.; Hwang, S. G.; Goddard, W. A., III Abstr. Pap., Am. Chem. Soc. 2004, 227, U850-U850.

[43] Jang, S. S.; Jang, Y. H.; Kim, Y.-H.; Goddard, W. A., III; Flood, A. H.; Laursen, B. W.; Tseng, H.-R.; Stoddart, J. F.; Jeppesen, J. O.; Choi, J. W.; Steuerman, D. W.; DeIonno, E.; Heath, J. R. J. Am. Chem. Soc. 2005, 127, 1563-1575.

[44] Jang, S. S.; Jang, Y. H.; Kim, Y. H.; Goddard, W. A., III; Choi, J. W.; Heath, J. R.; Laursen, B. W.; Flood, A. H.; Stoddart, J. F.; Norgaard, K.; Bjornholm, T. J. Am. Chem. Soc. 2005, 127, 14804-14816.

[45] Jang, Y. H.; Jang, S. S.; Goddard, W. A., III J. Am. Chem. Soc. 2005, 127, 4959-4964. 
[46] Jang, Y. H.; Goddard, W. A., III J. Phys. Chem. B 2006, 110, 7660-7665.

[47] Kim, Y.-H.; Goddard, W. A., III J. Phys. Chem. C 2007, 111, 4831-4837.

[48] Jeppesen, J. O.; Nygaard, S.; Vignon, S. A.; Stoddart, J. F. Eur. J. Org. Chem. 2004, 196, 220.

[49] Dichtel, W. R.; Heath, J. R.; Stoddart, J. F. Philos. Trans. R. Soc. A, Math. Phys. Eng. Sci. 2007, 365, 1607-1625.

[50] Carter, E. A.; Ciccotti, G.; Hynes, J. T.; Kapral, R. Chem. Phys. Lett. 1989, 156, 472-477.

[51] Sprik, M.; Ciccotti, G. J. Chem. Phys. 1998, 109, 7737-7744.

[52] Trzesniak, D.; Kunz, A. P. E.; van Gunsteren, W. F. ChemPhysChem 2007, 8, 162-169.

[53] Jaguar, V. 5.0 ed.; Schrödinger Inc.: Portland, 2003.

[54] Evans, D. J.; Morriss, G. P. Statistical Mechanics of Nonequilibrium Liquids; Academic Press: London, 1990.

[55] Fixman, M. Proc. Natl. Acad. Sci. U.S.A. 1974, 71, 3050-3053.

[56] Mayo, S. L.; Olafson, B. D.; Goddard, W. A., III J. Phys. Chem. 1990, 94, 8897-8909.

[57] Jang, S. S.; Molinero, V.; Cagin, T.; Goddard, W. A., III J. Phys. Chem. B 2004, 108, 31493157.

[58] Jang, S. S.; Lin, S.-T.; Cagin, T.; Molinero, V.; Goddard, W. A., III J. Phys. Chem. B 2005, 109, 10154-10167.

[59] Jang, S. S.; Goddard, W. A. J. Phys. Chem. C 2007, 111, 2759-2769.

[60] Jang, S. S.; Lin, S.-T.; Maiti, P. K.; Blanco, M.; Goddard, W. A., III; Shuler, P.; Tang, Y. J. Phys. Chem. B 2004, 108, 12130-12140.

[61] Jang, S. S.; Goddard, W. A. J. Phys. Chem. B 2006, 110, 7992-8001.

[62] Plimpton, S. J. J. Comput. Phys. 1995, 117, 1-19.

[63] Plimpton, S. J.; Pollock, R.; Stevens, M. The Eighth SIAM Conference on Parallel Processing for Scientific Computing Minneapolis; 1997.

[64] Swope, W. C.; Andersen, H. C.; Berens, P. H.; Wilson, K. R. J. Chem. Phys. 1982, 76, 637-649.

[65] Castro, R.; Nixon, K. R.; Evanseck, J. D.; Kaifer, A. E. J. Org. Chem. 1996, 61, 7298-7303. 
[66] Ashton, P. R.; Balzani, V.; Becher, J.; Credi, A.; Fyfe, M. C. T.; Mattersteig, G.; Menzer, S.; Nielsen, M. B.; Raymo, F. M.; Stoddart, J. F.; Venturi, M.; Williams, D. J. J. Am. Chem. Soc. 1999, 121, 3951-3957.

[67] Bryce, M. R.; Cooke, G.; Duclairoir, F. M. A.; Rotello, V. M. Tetrahedron Lett. 2001, 42, 1143-1145.

[68] Nielsen, M. B.; Jeppesen, J. O.; Lau, J.; Lomholt, C.; Damgaard, D.; Jacobsen, J. P.; Becher, J.; Stoddart, J. F. J. Org. Chem. 2001, 66, 3559-3563.

[69] Leigh, D. A.; Murphy, A.; Smart, J. P.; Deleuze, M. S.; Zerbetto, F. J. Am. Chem. Soc. 1998, $120,6458-6467$.

[70] Leigh, D. A.; Troisi, A.; Zerbetto, F. Chem. Eur. J. 2001, 7, 1450-1454.

[71] Deleuze, M. S.; Leigh, D. A.; Zerbetto, F. J. Am. Chem. Soc. 1999, 121, 2364-2379.

[72] Brough, B.; Northrop, B. H.; Schmidt, J. J.; Tseng, H. R.; Houk, K. N.; Stoddart, J. F.; Ho, C. M. Proc. Natl. Acad. Sci. U.S.A. 2006, 103, 8583-8588.

[73] Lin, S. T.; Blanco, M.; Goddard, W. A. J. Chem. Phys. 2003, 119, 11792-11805.

[74] Lin, S. T.; Jang, S. S.; Cagin, T.; Goddard, W. A. J. Phys. Chem. B 2004, 108, 10041-10052. 


\section{Chapter 4}

\section{Sodium Diffusion through Aluminum-Doped Zeolite BEA System: Effect of Water Solvation}

Reproduced with permission from Kim, H; Deng, W.-Q.; Goddard, W. A.; Jang S. S.; Davis M. E.; Yang Y. J. Phys. Chem. C 2009, 113, 819. Copyright 2009 American Chemical Society.

\subsection{Abstract}

To investigate the effect of hydration on the diffusion of sodium ions through the aluminum-doped zeolite BEA system $(\mathrm{Si} / \mathrm{Al}=30)$, we used the grand canonical Monte Carlo (GCMC) method to predict the water absorption into aluminosilicate zeolite structure under various conditions of vapor pressure and temperature, followed by molecular dynamics (MD) simulations to investigate how the sodium diffusion depends on the concentration of water molecules. The predicted absorption isotherm shows first-order-like transition, which is commonly observed in hydrophobic porous systems. The MD trajectories indicate that the sodium ions diffuse through zeolite porous structures via hopping mechanism, as previously discussed for similar solid electrolyte systems. These results show that above $15 \mathrm{wt} \%$ hydration (good solvation regime) the formation of the solvation cage dramatically increases sodium diffusion by reducing the hopping energy barrier by $25 \%$ from the value of $3.8 \mathrm{kcal} / \mathrm{mol}$ observed in the poor solvation regime.

\subsection{Introduction}

Zeolites constitute a unique class of the porous materials widely used in ion exchange, selective catalysis, and molecular sieve applications [1,2]. An important property for many of these applications is migration of the absorbed water molecules. It has been observed that water migration in such porous materials proceeds differently than in the bulk water phase under the same temperature and 
chemical potential conditions $[3,4,5,6]$; hence, we undertook a study of how nanoscale confinement in zeolites affects such of properties as structure, dynamics, and thermodynamics with absorbed molecules $[7,8,9,10,11,12,13,14,15,16,17,18]$.

In aluminosilicate zeolites the aluminum is generally incorporated in the three-dimensional framework in the form of $\mathrm{AlO}_{4}^{-}$surrounded by neighboring $\mathrm{SiO}_{4}$ while alkali metals such as $\mathrm{Na}^{+}$or $\mathrm{K}^{+}$ are in the pores, interacting electrostatically with the zeolite framework. These positively charged and Movable cations in various zeolite systems have been studied intensively [7, 8, 19, 20, 21, 22, $23,24,25,26,27,28,29]$ since they impart many interesting properties to the zeolite systems. In particular, the ionic conductivity of zeolites can be controlled by the level of nonframework ions and their hydration (undoped zeolites are insulators with an electronic band gap of $\sim 7 \mathrm{eV}$ ) [9]. The chemical nature, hydrophilicity, and selectivity for binding guest molecules in zeolites can be manipulated as appropriate for many practical applications [10, 11].

One recent interesting application of zeolites is as proton exchange membranes for fuel cells (PEMFC) $[12,13,14,15,16,17]$. Here the molecular sieving capability and tunable acidity/hydrophilicity of zeolites can be incorporated to form a polyelectrolyte-based membrane that could improve the high temperature/low humidity performance of fuel cells [18].

We report here studies of the aluminosilicate zeolite $\mathrm{BEA}(\mathrm{Si} / \mathrm{Al}=30)$. A distinct structural feature of interest for BEA is its three-dimensional channel structure which is characterized by $\sim 1$ nm diameter channels (Figure 4.1) in which water molecules may form a continuous phase, thereby facilitate ionic transport which makes membranes based on such materials useful as a replacement for polymer electrolyte membranes (PEM) for fuel cell applications. In PEM fuel cell membrane such at Nafion, the protons are transported through two mechanisms: vehicular diffusion of protonated waters and Grotthuss diffusion in which protons hop from water to water, with the relative contributions depending on the water content, counterions, porosity, temperature etc. Of course, diffusion of sodium or potassium cations occurs only through hopping. In this study, we determine how the nanometer scale confinement in the aluminosilicate zeolite BEA ( $\mathrm{Si} / \mathrm{Al}=30)$ affects sodium diffusion.

First, we used grand canonical Monte Carlo (GCMC) simulations to predict the water absorption of aluminosilicate zeolite BEA system at various conditions, and then we applied the equilibrium molecular dynamics (MD) simulations on the diffusion starting with the hydrated systems obtained from the GCMC simulations. During such MD simulations, we sampled the time evolution of both the sodium diffusion and the water structure associated with the sodium ions. 

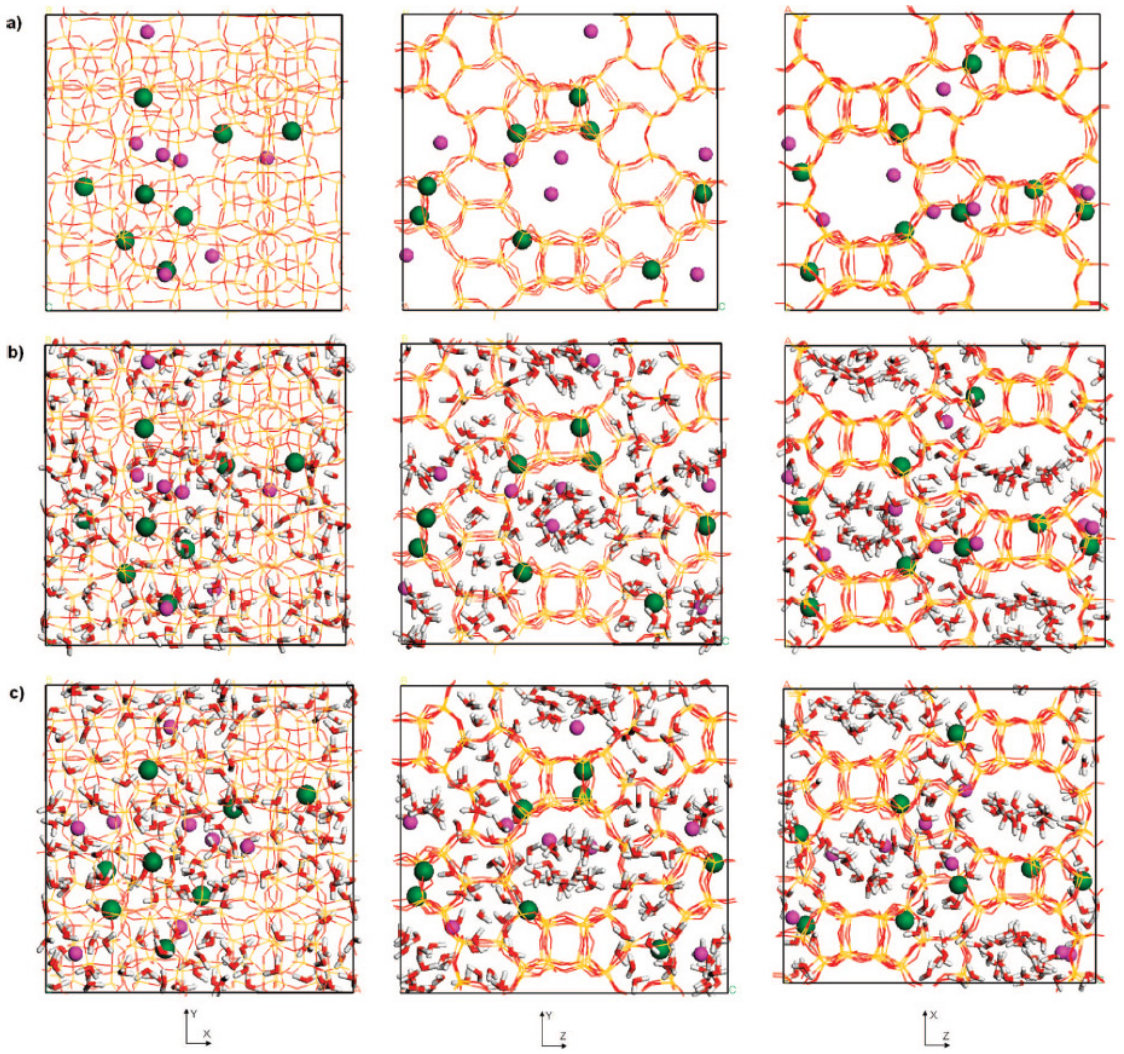

Figure 4.1: Atomistic structure for the primitive periodic cell with composition of $\mathrm{Na}_{8} \mathrm{Al}_{8} \mathrm{Si}_{248} \mathrm{O}_{512}$. This shows green balls are aluminum and magenta balls are sodium. (a) Minimized aluminosilicate zeolite BEA structures without $\mathrm{H}_{2} \mathrm{O}$, (b) snapshot of zeolite BEA structures after the GCMC simulation for $298.15 \mathrm{~K}$ and $101.3250 \mathrm{kPa}$, and (c) snapshot of zeolite BEA structures after $4 \mathrm{~ns}$ NPT MD simulation at $298.15 \mathrm{~K}$ and $101.3250 \mathrm{kPa}$. 


\subsection{Simulation Details}

\subsubsection{Force Field}

To calculate the energy and geometry of the zeolite framework, we employed Burchart force field (FF) [19] developed to describe aluminosilicate structures and the van der Waals interactions with the Na. This FF uses the Lennard-Jones $12-6$ form for the van der Waals interactions between all atoms of the framework. To describe the interactions between water molecules, we used the F3C water FF [20]. For the off-diagonal van der Waals interactions between different types of atoms, we used the geometric-mean combination rule. The Lennard-Jones interactions were terminated beyond $18.0 \AA$, using a cubic spline function starting at $15.0 \AA$.

The atomic charges of the individual atoms of the zeolite frame were determined using the charge equilibration (QEq) method [21]. Since our simulation cell contains eight $\mathrm{AlO}_{4}^{-}$sites, the total charge of the framework is set by -8 . The eight sodium ions each were taken to have +1 charge, leading to charge neutrality of the simulation cell. The atomic charge of the water is from the F3C model [20]. The particle-particle particle-mesh (PPPM) method [22] was employed to compute the electrostatic using an accuracy criterion of $10^{-5}$.

\subsubsection{Grand Canonical Monte Carlo (GCMC) method and Molecular Dy- namics (MD) Simulation}

To predict the water absorption in aluminosilicate zeolite BEA framework as a function of partial pressure and temperature, we carried out grand canonical Monte Carlo (GCMC) simulations [23, 24] using the Sorption module of Cerius2 [25]. Since the chemical potential of the adsorbed phase equals the chemical potential of the bulk gas at equilibrium condition, the GCMC simulation makes multiple attempts to add one water molecule according to the probability

$$
P_{a d d}=\min \left[1, \frac{V}{(N+1) \Lambda^{3}} \exp \left(-\frac{\Delta U}{k_{B} T}\right)\right],
$$

or subtract one with the following probability

$$
P_{s u b}=\min \left[1, \frac{N \Lambda^{3}}{V} \exp \left(-\frac{\Delta U}{k_{B} T}\right)\right],
$$

where $V$ is the pore volume, $N$ is the number of water molecules in the simulated system, $\Lambda$ is the thermal de Broglie wavelength, and $\Delta U$ is the change in potential energy. For each GCMC simulation, we ran $2 \times 10^{8}$ Monte Carlo steps (MCS), during which the zeolite framework including sodium ions were fixed and water molecules were moved by the Metropolis sampling rule [26, 27]. Each Monte Carlo step is allowed four different types of operations-translation, rotation, creation, 
and destructions-of the water molecule with equal probabilities.

All molecular dynamics (MD) simulations were performed using the LAMMPS (large-scale atomic/molecular massively parallel simulator) MD code developed by Plimpton at Sandia [28, 29]. The velocity Verlet algorithm[30] was used to solve the equations of motion with a time step of 1.0 fs. The isobaric-isothermal ensembles (NPT) were generated using Nosé-Hoover thermostat with a temperature damping relaxation time of 0.1 ps and the Andersen-Hoover barostat with a dimensionless cell mass factor of 1.0. A series of MD simulations were conducted at constant temperature of 298.15 K with various pressure conditions from 0.1013 to $101.3250 \mathrm{kPa}$. To understand the effect of temperature, another set of simulations were performed at constant pressure of $101.3250 \mathrm{kPa}$ with various temperature conditions from 298.15 to $453.15 \mathrm{~K}$.

\subsubsection{Construction of Models and Calculation of Properties}

We used the $2 \times 2 \times 1$ superstructure of zeolite BEA (based on the X-ray crystallography database [31]) as the periodic simulation cell for the MD. Then, eight silicon atoms in the channels were randomly chosen and replaced with aluminum atoms to have the lowest energy using substitutional disorder option of Cerius2 [25]. This leads to a $\mathrm{Si} / \mathrm{Al}$ ratio of 30, with a simulation cell consisting of $8 \mathrm{AlO}_{4}^{-}$and $248 \mathrm{SiO}_{4}$ tetrahedral sites with an overall composition of $\mathrm{Na}_{8} \mathrm{Al}_{8} \mathrm{Si}_{248} \mathrm{O}_{512}$.

The eight sodium ions were initially placed near the aluminum-doped sites, and the full structure of zeolite frame including the sodium ions were energy minimized. Then the water contents were determined from GCMC simulations at various pressure and temperature conditions.

Then we carried out 8-12 ns NPT MD simulations and evaluated such properties as density, pair correlation function, and diffusion coefficients using the full trajectory files.

\subsection{Results and Discussion}

\subsubsection{Water Absorption}

Using GCMC, the chemical potential of water in zeolite frame was equilibrated with that of the external reservoir at various vapor pressure conditions ranging from 0.1013 to $101.3250 \mathrm{kPa}$. The water absorption isotherm at $298.15 \mathrm{~K}$ and the temperature dependence of water adsorption at $101.3250 \mathrm{kPa}$ are shown at Figures 4.2 and 4.3 , respectively.

We were unable to locate published water adsorption isotherms for zeolite BEA with $\mathrm{Si} / \mathrm{Al}$ of 30. Thus the data shown in Figures 4.2 and 4.3 are from simulation only. We observe a stepwise condensation at $3.5 \mathrm{kPa}$. The amount of water uptake increases abruptly and then shows fast saturation up to $\sim 50$ molecules per crystallographic unit cell, which can be regarded as a maximum loading number at $298.15 \mathrm{~K}$. Previous studies discovered that a spontaneous condensation of water 


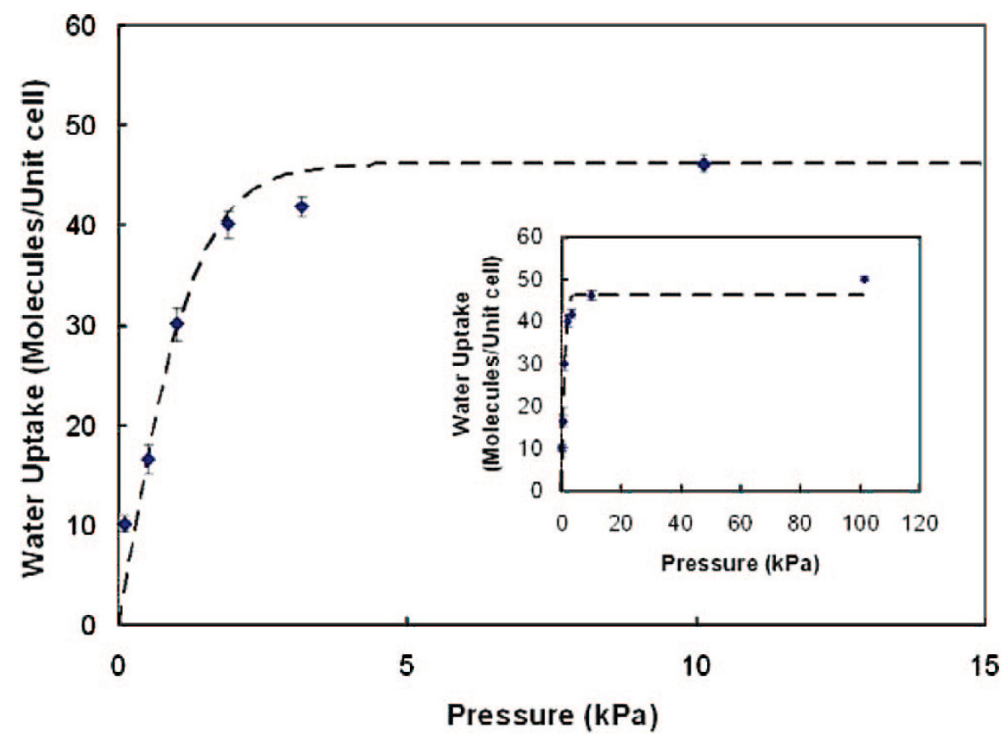

Figure 4.2: Dashed line is the least-squares fitted to $f(x)=a \tanh (b x)$, where $a=46.19$ molecules/ unit cell and $b=0.753(1 / \mathrm{kPa})$. We obtain a vapor pressure of $3.578 \mathrm{kPa}$ for the point at which the water uptake reaches to $99 \%$ of the maximum loading uptake.

occurs in hydrophobic porous materials such as sodium faujasites of $\mathrm{NaY}$ and $\mathrm{NaX}$ [32], silicate-1 zeolites [33, 34] and carbon nanotubes [35]. Here, the term "hydrophobic" is used because of the spontaneous condensation behavior: if the surface of the nanopore were hydrophilic, then water absorption would proceed gradually over wide range of pressure through wetting the surface of the nanopore instead of increasing abruptly at a certain narrow pressure range. We expect that the capillary condensation would follow a first-order-like transition since the nonwetting character of the pore prohibits the intrusion of liquid water until a certain hydraulic pressure. This suggests that the aluminosilicate BEA zeolite system may sustain some extent of hydrophobicity even after some of the hydrophobic $\mathrm{SiO}_{4}$ sites are replaced by hydrophilic $\mathrm{AlO}_{4}^{-}$sites.

The temperature dependence of water absorption is plotted at Figure 4.3 at $101.3250 \mathrm{kPa}$. As the temperature increases, the amount of water uptake in zeolite frame decreases. This type of temperature dependency of water uptake has been observed in the experiments on the zeolite-4A structure [36] and in other simulation studies [32]. Of particular interest is that at $423 \mathrm{~K}$, this BEA aluminosilicate zeolite frame still holds $20 \%$ of water absorbed at $300 \mathrm{~K}$. We expect that this capability to retain significant amount of water at high temperature could make these materials useful as a replacement for performance of the PEM for fuel cell operation under high temperature/low humidity condition. 


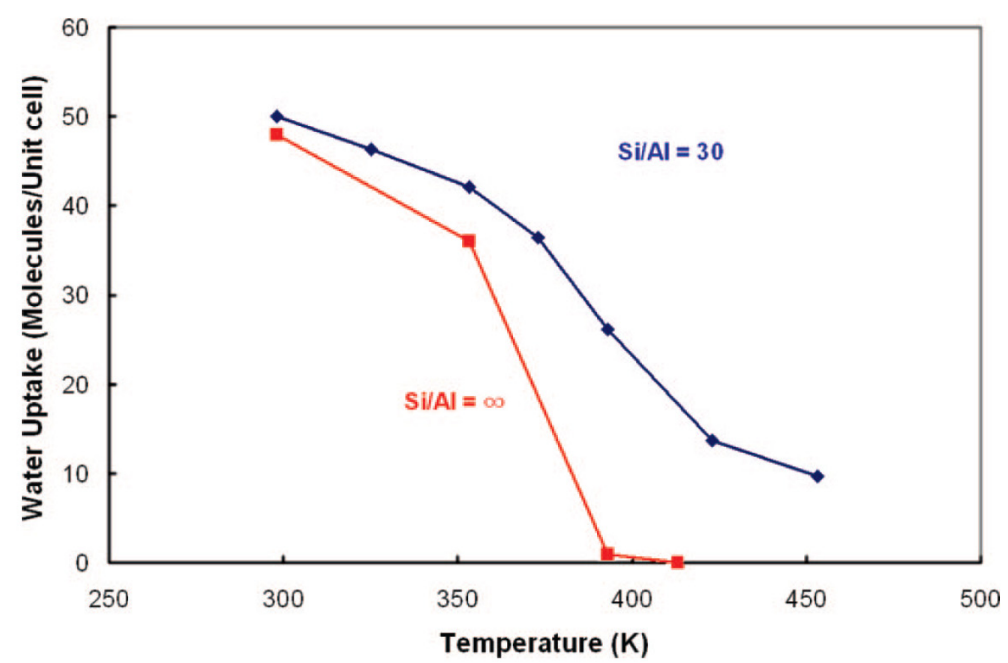

Figure 4.3: Predicted temperature-dependence of water uptake at $101.3250 \mathrm{kPa}$ when the zeolite $\mathrm{BEA}$ has no aluminum doping (red curve) and $\mathrm{Si} / \mathrm{Al}=30$ (blue curve). The presence of aluminumdoped sites as well as sodium ions enhance the water uptake amount especially in the high temperature.

\subsubsection{Structure of Water in Zeolite}

To characterize the water structure we calculated the density-normalized $\mathrm{O}$ (water)-O(water) pair correlation function, $4 \pi r^{2} \rho g_{O}$ (water) $-O($ water $)$ where $r$ is the distance and $\rho$ is the number density of water. Figure 4 .4a shows $4 \pi r^{2} \rho g_{O \text { (water)-O(water) }}$ plots for water absorbed in various vapor pressure conditions and compares to the values for bulk water. Integrating the first peak in Figure 4.4a leads to the coordination number $\mathrm{CN}$. For bulk water we obtain $\mathrm{CN}=4.59$, in good agreement with $\mathrm{CN}=4.5$, from neutron diffraction experiment [37]. Figure 4.4b summarizes the change of the coordination numbers as a function of pressure, showing a behavior identical with the absorption isotherm in Figure 4.2. This provides clear evidence for the spontaneous condensation in which all the water molecules absorbed into the system participate in water clusters. Figure 4.4 shows that the structure of the water absorbed in the zeolite framework approaches to that of bulk phase water as the water uptake/pressure is increased to $101.3250 \mathrm{kPa}$. However, the saturated value of $\mathrm{CN}$ of the absorbed phase $(\sim 3.8)$ is smaller than the $\mathrm{CN}$ of the bulk phase $(4.5)$. This is reasonable because of the huge surface area of $1362.99 \mathrm{~m}^{2} / \mathrm{g}$ of the nanopores of the zeolite (cf. activated carbon has $500-1500 \mathrm{~m}^{2} / \mathrm{g}$ ). We observed similar behavior for water in Nafion and Dendrion polymer membranes $[38,39,40]$.

In order to investigate the effect of the sodium ion on the water structure, we analyzed the density-normalized Na-O(water) pair correlation function, $4 \pi r^{2} \rho g_{N a-O(w a t e r)}$ and the water coordination number of sodium ion as shown in Figure 4.5, parts a and b, respectively. Similar to $4 \pi r^{2} \rho g_{O \text { (water)-O(water) }}$ in Figure 4.4 we see that $4 \pi r^{2} \rho g_{N a-O(w a t e r)}$ increases with increasing pressure with a first peak position that does not change with pressure. However, the increase of the 

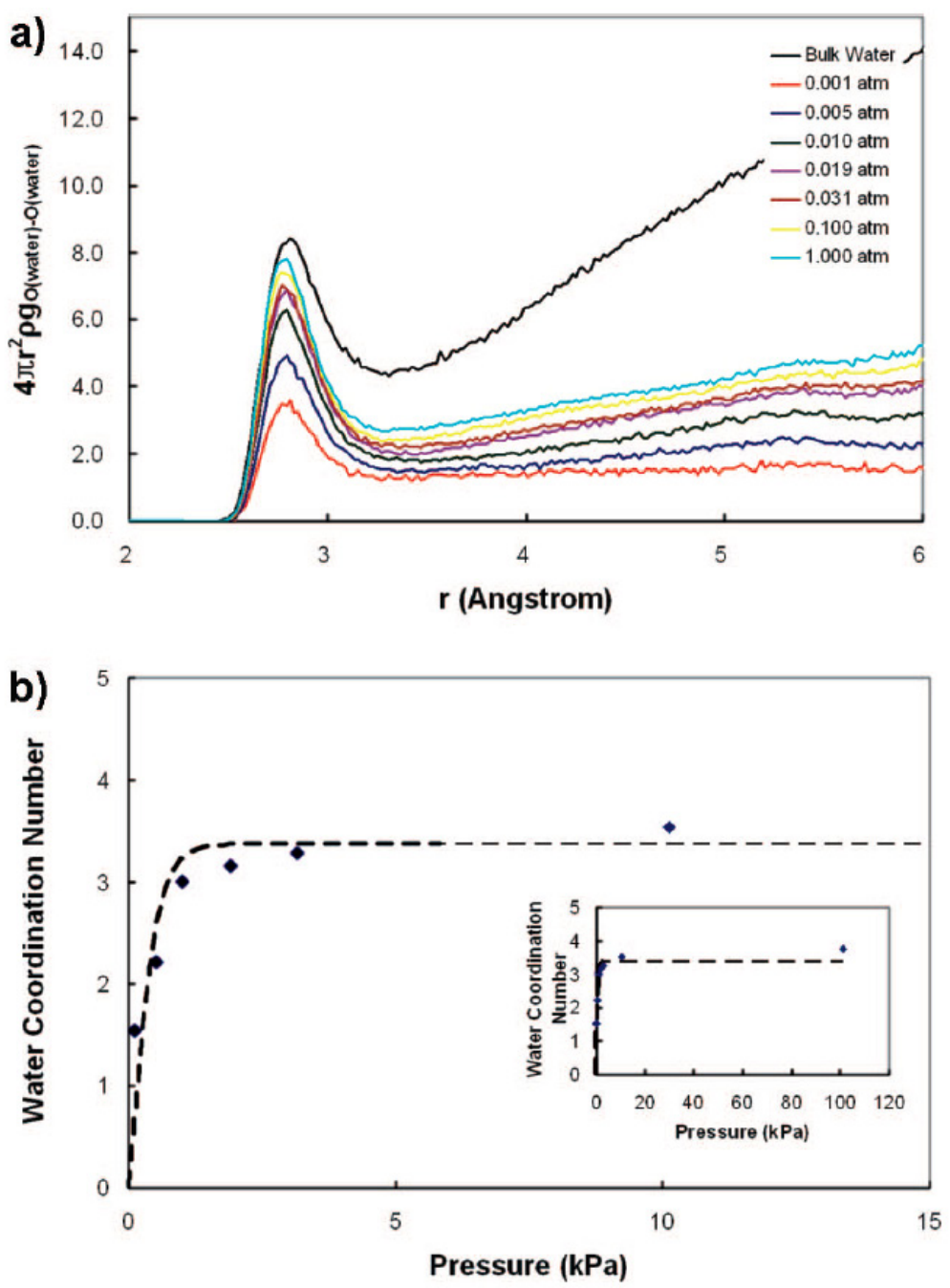

Figure 4.4: (a) Probability of finding additional water molecules at a specific distance from each water molecule. This is obtained as the product of pair correlation functions of water oxygens, $g_{O(\text { water })-O(\text { water })}$ with water density $\rho$ and $4 \pi r^{2}$. (b) Water coordination number (CN) of water molecule as a function of pressure. The $\mathrm{CN}$ of water molecule is obtained from the integration of curve a up to first minimum, which is at $3.7 \AA$. The saturated value of CN of the absorbed phase $(\sim 3.8)$ is smaller than the $\mathrm{CN}$ of the bulk phase $(4.5)$. 
first peak intensity of $4 \pi r^{2} \rho g_{N a-O \text { (water) }}$ up to $\sim 3.5 \AA$ was not significant compared to that of

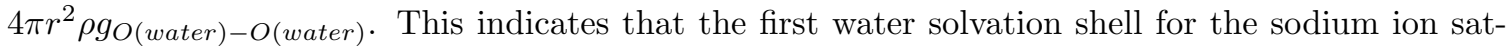
urates rapidly toward $\mathrm{CN}=\sim 5.0$ (see Figure 5b inset), which is consistent with the previous indication that water molecules cluster with sodium rather than interact with the surface of the nanopore, (indicating a hydrophobic nature of the nanopore surface). We believe that this is because the strong interaction of the net charge in the sodium ion enhances the development of its solvation structure. This value shows excellent agreement with the experiment which finds 4.82 [41].

\subsubsection{Effect of Water Contents on Sodium Diffusion}

The diffusion coefficient of the sodium ion was calculated at pressures ranging from 0.1013 to 101.3250 $\mathrm{kPa}$, as summarized at Table 4.1. To characterize the nature of the diffusion, Figure 4.6 shows the $\log -\log$ plot of the mean square displacements (MSD) versus time. For times up to $~ 20$ ps, the MSD increases as a function of $t^{1 / 2}$ indicating single file diffusion (SFD) behavior. For simulation times longer than $20 \mathrm{ps}$, the MSD shows a normal Fickian behavior $(\mathrm{MSD} \propto t)$. This transition of MSD from SFD to normal Fickian diffusion occurs when the pore confines the particles but still allows the particles to pass by each other [42]. In the long time Fickian regime, we obtained the diffusion coefficient $D$ from the mean square displacements of the sodium ions using Equation 4.3,

$$
D=\left\langle(r(t)-r(0))^{2}\right\rangle / 6 t
$$

In order to examine how the dynamics of sodium ion is influenced by its water solvation shell, we analyzed the diffusion coefficient of sodium ion versus water coordination number at 298.15 K. From Figure 4.7, we observed a dramatic jump at about $\mathrm{CN}=4.5$ that corresponds to the point of the water uptake saturation in the absorption isotherm (Figure 4.2). Indeed, Faux and his coworkers $[43,44,45]$ reported theoretical studies that the diffusion coefficient of sodium in zeolite-4A system increases from $1 \times 10^{-7} \mathrm{~cm}^{2} / \mathrm{s}$ to $10 \times 10^{-7} \mathrm{~cm}^{2} / \mathrm{s}$ as the number of water molecules per unit cell increases from 0 to 224 in agreement with the trend and order of magnitude of the diffusion coefficient in our simulations. Furthermore, Faux et al. also observed a sudden jump of the sodium diffusion coefficient between 112 and 168 water molecules per unit cell, but did not explain the origin. We believe that this results directly from the degree of solvation. This spontaneous condensation of water (discussed in Section 4.4.1), leads to two regimes for the ionic diffusion in the presence of water. Detailed analysis of sodium ion trajectories allows us to enunciate why a threshold amount of water uptake is critical to enhance the diffusion of the ion from the microscopic point of view. The doped $\mathrm{AlO}_{4}^{-}$sites are Brönsted acid sites [46] that bound the nonframework cations via strong Coulomb interaction. Parts a and b of Figure 4.8 show the time profile of the Al-Na distances for the good solvation regime (vapor pressure $=101.3250 \mathrm{kPa}$ ) and the poor solvation regime (vapor 

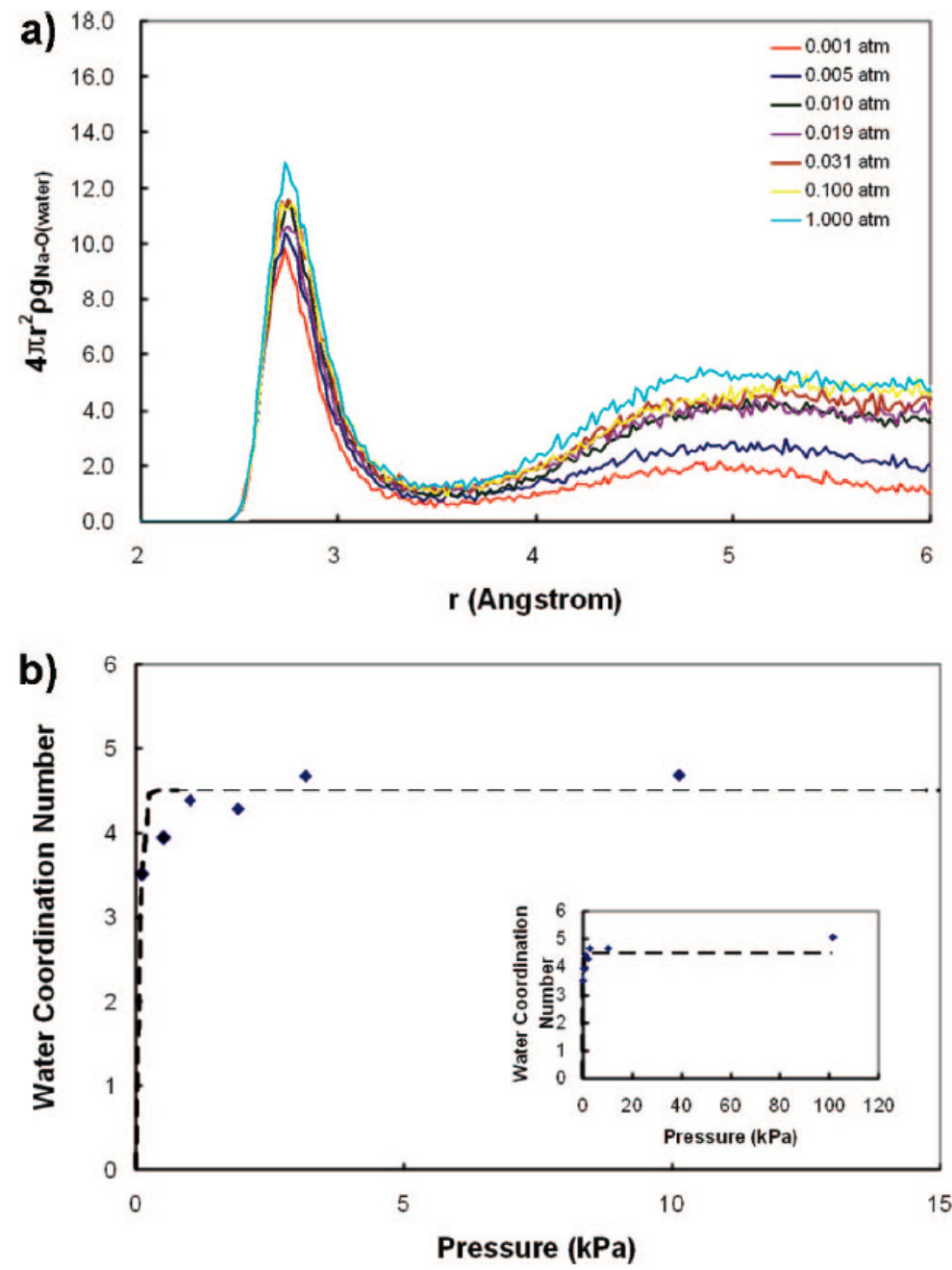

Figure 4.5: (a) Probability of finding sodium ions at a specific distance from each water molecule. This is obtained as the product of pair correlation functions of sodium atoms and water oxygen, $g_{N a-O \text { (water })}$ with water density $\rho$ and $4 \pi r^{2}$. (b) Water coordination number $(\mathrm{CN})$ of sodium ions as a function of pressure. The $\mathrm{CN}$ of water molecule is obtained from the integration of curve a up to first minimum, which is at $3.5 \AA$. The saturated value of $\mathrm{CN}$ of the absorbed phase $(\sim 5.0)$ is same to the $\mathrm{CN}$ of the bulk phase. 


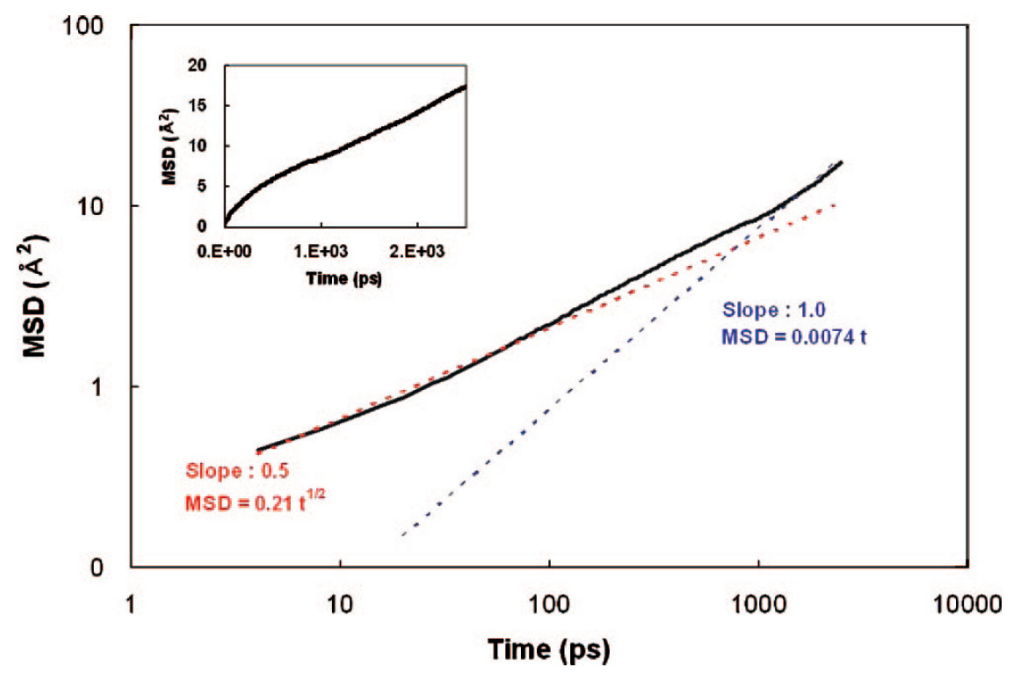

Figure 4.6: Log-log plot of mean square displacements (MSD) vs time at 298.15 K and 101.3250 $\mathrm{kPa}$ based on a trajectory of $12 \mathrm{~ns}$. Initially the MSD shows MSD $\propto t^{1 / 2}$ up to $t=\sim 20 \mathrm{ps}$, but the times $>\sim 2 \mathrm{~ns}$, we see normal $3 D$ Fickian behavior (MSD $\propto t$ ). The Fickian regime leads to a diffusion coefficient of $D=1.233 \times 10^{-7} \mathrm{~cm}^{2} / \mathrm{s}$.

Table 4.1: Vapor Pressures, Water Uptake/Cell, Water Coordination Numbers of the Water Molecule (up to $3.7 \AA$ Cutoff), Water Coordination Numbers of the Sodium Ion (up to $3.5 \AA$ Cutoff), and Diffusion Coefficients from NPT MD at Temperature of $298.15 \mathrm{~K}$

\begin{tabular}{ccccc}
\hline \hline $\begin{array}{c}\text { vapor pressure } \\
(\mathrm{kPa})\end{array}$ & water uptake/cell & $\begin{array}{c}\mathrm{CN} \\
(\text { water })\end{array}$ & $\begin{array}{c}\mathrm{CN} \\
(\text { sodium })\end{array}$ & $\begin{array}{c}\text { diffusion coefficient } \\
\left(\mathrm{cm}^{2} / \mathrm{s}\right)\end{array}$ \\
\hline 0.1013 & 10.15 & 1.55 & 3.51 & $(0.338 \pm 0.033) \times 10^{-7}$ \\
0.5066 & 16.57 & 2.22 & 3.94 & $(0.382 \pm 0.048) \times 10^{-7}$ \\
1.0133 & 30.08 & 3.01 & 4.39 & $(0.642 \pm 0.219) \times 10^{-7}$ \\
1.9006 & 40.10 & 3.16 & 4.29 & $(1.431 \pm 0.041) \times 10^{-7}$ \\
3.1677 & 41.86 & 3.29 & 4.67 & $(1.170 \pm 0.035) \times 10^{-7}$ \\
10.1325 & 46.18 & 3.54 & 4.68 & $(1.478 \pm 0.368) \times 10^{-7}$ \\
101.3250 & 50.00 & 3.77 & 5.04 & $(1.466 \pm 0.601) \times 10^{-7}$ \\
\hline \hline
\end{tabular}

pressure $=0.1013 \mathrm{kPa}$ ), respectively. This shows clearly that each sodium ion localizes within $\sim 5$ $\AA$ of one of the aluminum-doped sites and diffuses by hopping from one site to a nearest neighbor aluminum site. Thus, the diffusion of sodium ion occurs through the hopping mechanism between $\mathrm{AlO}_{4}^{-}$sites (the Brönsted acid sites) with the energy barrier of $3.5-4.0 \mathrm{kcal} / \mathrm{mol}$ caused by the electrostatic interaction. Figure 4.8a indicates that the hopping events occur every 2 ns on average in the good solvation regime, whereas Figure 4.8b shows just 1 hop within $12 \mathrm{~ns}$ in the poor solvation regime. This explains why the diffusion coefficient of sodium ion is $\sim 10$ times larger in the good solvation regime than in the poor solvation regime as in Table 4.1. Sufficient numbers of water molecules are the most critical in creating solvation cage and helping the ionic hopping. 


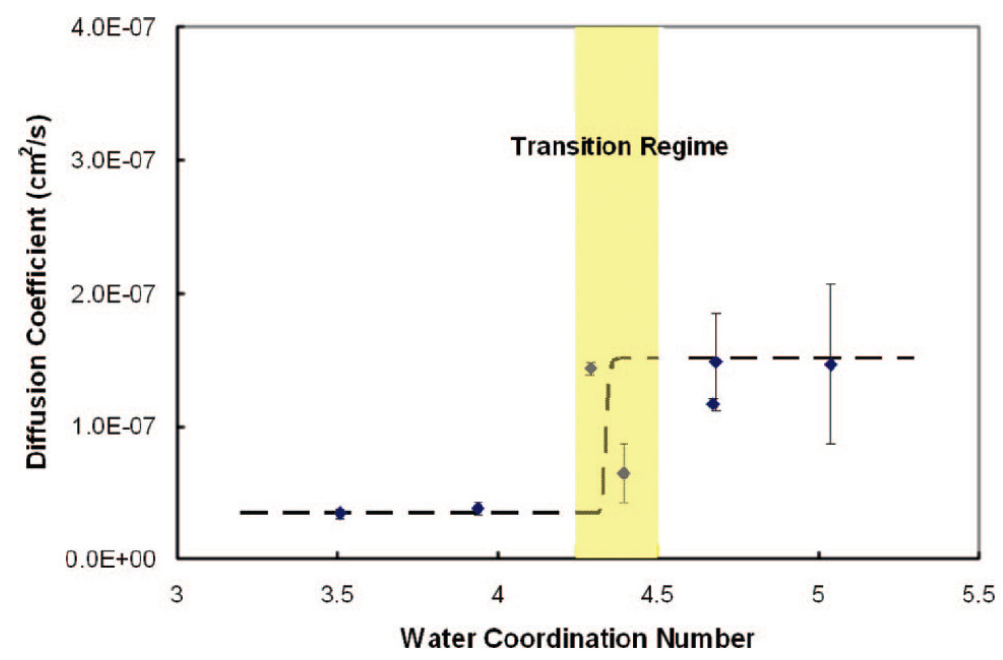

Figure 4.7: Dependence of diffusion coefficient of sodium ion on water coordination number $(\mathrm{CN})$ of sodium ion. The diffusion coefficients were calculated from three partitioned trajectories of 12 ns NPT simulations (each partition has $4 \mathrm{~ns}$ length), and the error bars were evaluated from the diffusion coefficients obtained from the three partitioned trajectories. This represents pressures from 0.1013 to $101.3250 \mathrm{kPa}$ at $298.15 \mathrm{~K}$. At a pressure of $\sim 2.0 \mathrm{kPa}$, we find an abrupt change in the water $\mathrm{CN}$. When the sodium ion is solvated by $>4.5$ water molecules, its diffusion coefficient increases abruptly by a factor of 3.5. The dashed line is to guide the eye.

\subsubsection{Effect of Temperature on Sodium Diffusion}

We calculated the diffusion coefficients of the sodium ions confined in the aluminosilicate zeolite BEA membrane are obtained for various temperatures ranging $298.15 \mathrm{~K}$ to $453.15 \mathrm{~K}$. For each temperature, the amount of water uptake was determined using GCMC simulation (Figure 4.3). The temperature effect on the sodium diffusion was taken from MD simulations based on the hydrated zeolite. These diffusion coefficients are presented in Table 4.2 and Figure 4.9. Up to $373.15 \mathrm{~K}$, the diffusion coefficient of sodium ion increases with increasing temperature, as expected for a normal activated process. However, we observe from Figure 4.9 that the diffusion coefficient decreases with increasing temperature from $373.15 \mathrm{~K}$ to $453.15 \mathrm{~K}$. This anomaly is a consequence of the less hydration of the zeolite at high temperature as previously discussed in Section 4.4.2. Here, the insufficient water absorption results in a less developed solvation shell, which cannot facilitate the sodium ion hopping events. Thus, the diffusion coefficient decreases at high temperature range with less number of water molecules. Therefore, just as in the discussion about good solvation and poor solvation regimes that depend on the vapor pressure, we consider that the good solvation regime applies up to $373.15 \mathrm{~K}$, while the poor solvation regime applies beyond $400 \mathrm{~K}$ (the $393.15 \mathrm{~K}$ point lies within the transition regime.)

Normally, the temperature dependence for diffusion coefficient is written in terms of a standard Arrhenius equation, Equation 4.4. 

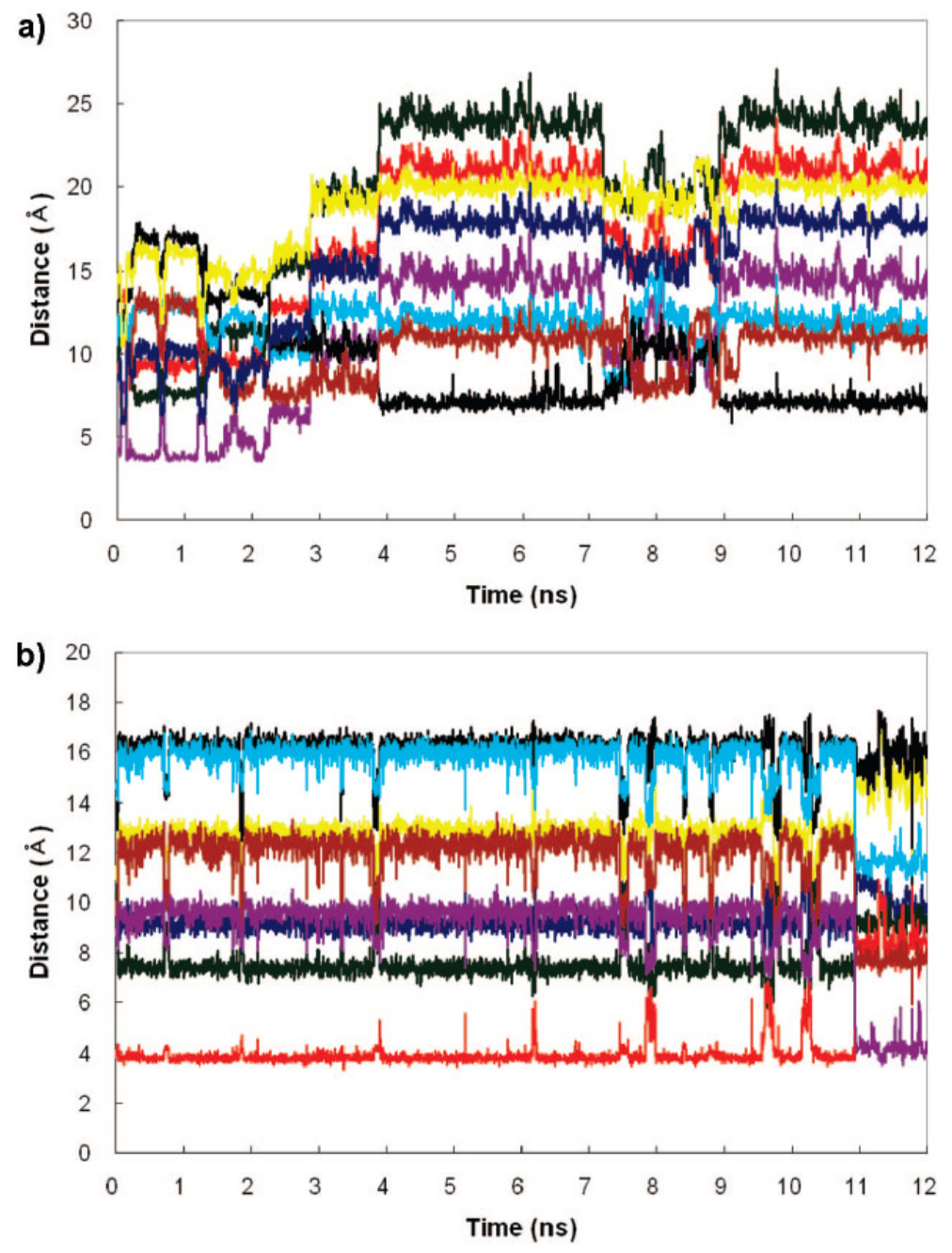

Figure 4.8: Time dependence of the distances of one sodium ion to all 8 aluminum atoms of the zeolite membrane. The distance to each aluminum atom is shown with a different color. (a) Vapor pressure is $101.3250 \mathrm{kPa}$ (good solvation regime) at $298.15 \mathrm{~K}$. This shows that it took $2 \mathrm{~ns}$ for the $\mathrm{Na}$ to hop from a position $4.8 \AA$ from the purple $\mathrm{Al}$ to a position $7.5 \AA$ from the black Al. Here a distance of $4.8 \AA$ indicates one water shell between the $\mathrm{Na}$ and the Al, while $7.5 \AA$ indicates separate water shells around each; (b) vapor pressure is $0.1013 \mathrm{kPa}$ (poor solvation regime) at $298.15 \mathrm{~K}$. In this case the $\mathrm{Na}$ remains at $4 \AA$ from the $\mathrm{Al}$ which indicates that the $\mathrm{Na}$ remains coordinated to the $\mathrm{O}$ of the $\mathrm{Al}$. 


$$
D=A_{\text {diff }} \exp \left(-\frac{\Delta E}{k_{B} T}\right)
$$

where the $\Delta E$ implies the energy barrier for the hopping from one site to another site. $A_{\text {diff }}=$ $L^{2} k_{B} T / h \exp (n s / R)$ is the prefactor, a product of frequency factor (activation entropy term) with a hopping-related length $(L) . k_{B}$ is the Boltzmann constant and $T$ is the absolute temperature. In order to include the solvation effect, we define the stabilization energy $\Delta E_{\text {solv }}$ due to the solvation. This leads to a modified Equation 4.4 where $A_{\text {diff-solve }}$ now includes a correction for solvation.

$$
D=A_{\text {diff-solve }} \exp \left(-\frac{\Delta E-\Delta E_{\text {solv }}}{k_{B} T}\right) .
$$

We assume that the $\Delta E_{\text {solv }}$ is a step function which is zero for the poor solvation regime and nonzero for the good solvation regime, which implies that the incomplete solvation shell of sodium ion has no effect to stabilization. The Arrhenius plot for each regime is shown in Figure 4.10 and the curve for each regime is fitted with a linear equation using least-squares fitting method. From the slope of the fitted line, we estimate the $\Delta E=3.810 \mathrm{kcal} / \mathrm{mol}$ for the poor solvation regime and the $\Delta E-\Delta E_{\text {solv }}=3.540 \mathrm{kcal} / \mathrm{mol}$ for the good solvation regime. Thus, the stabilization energy by solvation $\left(\Delta E_{\text {solv }}\right)$ becomes $0.270 \mathrm{kcal} / \mathrm{mol}$. The $y$-intercepts lead to $A_{\text {diff }}=-10.70 \mathrm{~cm}^{2} / \mathrm{s}$ and $A_{\text {diff-solve }}=-9.76 \mathrm{~cm}^{2} / \mathrm{s}$. The nearly unit value for $A$ suggests that the diffusion mechanism for both regimes is basically identical, supporting the Equation 4.5. Although no experimental data has been reported for the energy barrier of the diffusion of sodium ion, the energy barrier for the diffusion of sodium ion in the $\beta$ alumina (known as a good ionic conductor) has been measured experimentally $[47,48]$. Since the sodium ion in the $\beta$ alumina is also believed to jump around aluminum oxide sites, these values should be comparable with ours. Kim et al. [47] reported that the barrier as $3.275 \mathrm{kcal} / \mathrm{mol}$ from the measurement of ionic conductivity and $3.897 \mathrm{kcal} / \mathrm{mol}$ from the measurement of sodium tracer diffusion. Also the values from Whittingham and Huggins [48] obtained values of $3.81 \mathrm{kcal} / \mathrm{mol}$ from the tracer diffusion measurement and $3.95 \mathrm{kcal} / \mathrm{mol}$ from the dielectric loss measurement. All these values are very comparable to our $\Delta E$ value of $3.540 \mathrm{kcal} / \mathrm{mol}$, supporting the accuracy of our simulations. On the basis of these comparable values of the energy barrier, we expect that the stabilization energy for the hydrated system would also be reasonable although the effect of hydration in such aluminosilicate system has yet been reported.

\subsection{Conclusions}

Combining GCMC and MD simulations, we studied the effect of hydration on the sodium dynamics. From the absorption isotherm, we observed that the water absorption in BEA zeolites proceeds with a spontaneous condensation at a pressure of $3.5 \mathrm{kPa}$ and a temperature of $298.15 \mathrm{~K}$ even though 
Table 4.2: Temperature, Water Uptake/Cell, and Diffusion Coefficients at Constant Pressure Condition of $101.3250 \mathrm{kPa}$

\begin{tabular}{ccccc}
\hline $\begin{array}{c}\text { temperature } \\
(\mathrm{K})\end{array}$ & water uptake/cell & $\begin{array}{c}\mathrm{CN} \\
\text { (water) }\end{array}$ & $\begin{array}{c}\text { CN } \\
(\text { sodium })\end{array}$ & $\begin{array}{c}\text { diffusion coefficient } \\
\left(\mathrm{cm}^{2} / \mathrm{s}\right)\end{array}$ \\
\hline 298.15 & 50.00 & 3.77 & 5.04 & $(1.466 \pm 0.601) \times 10^{-7}$ \\
325.15 & 46.25 & 3.73 & 4.71 & $(2.372 \pm 0.024) \times 10^{-7}$ \\
353.15 & 42.12 & 3.72 & 4.24 & $(3.762 \pm 0.822) \times 10^{-7}$ \\
373.15 & 36.50 & 3.46 & 4.39 & $(4.806 \pm 0.226) \times 10^{-7}$ \\
393.15 & 26.17 & 3.27 & 4.08 & $(3.725 \pm 0.188) \times 10^{-7}$ \\
423.15 & 13.73 & 2.45 & 3.63 & $(2.630 \pm 0.459) \times 10^{-7}$ \\
453.15 & 9.75 & 1.74 & 3.42 & $(3.042 \pm 0.301) \times 10^{-7}$ \\
\hline \hline
\end{tabular}

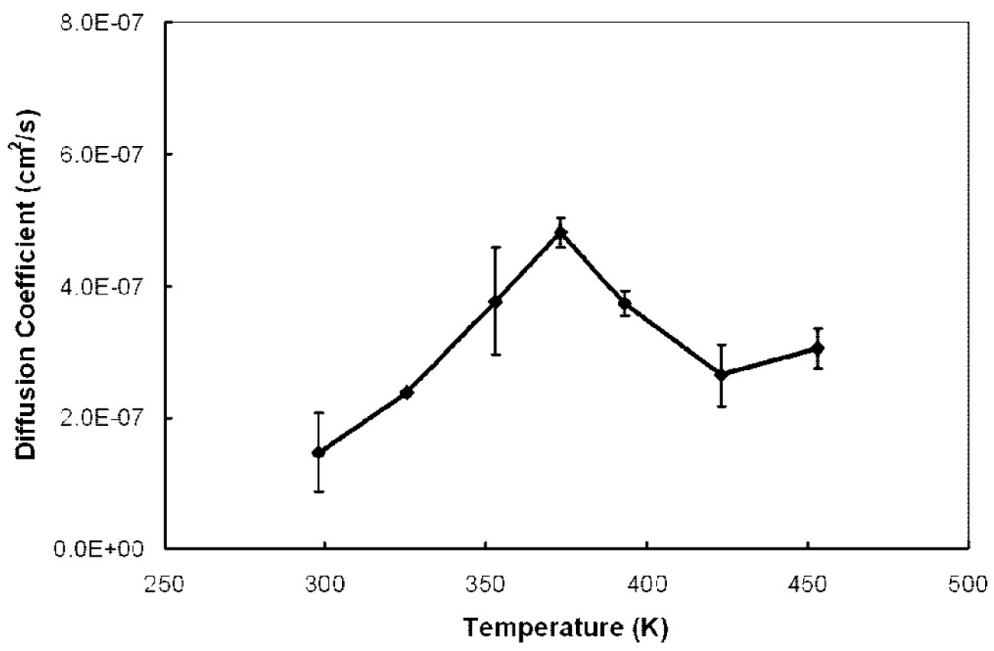

Figure 4.9: Dependence of the sodium diffusion coefficient on the temperature for a vapor pressure of $101.3250 \mathrm{kPa}$. The diffusion coefficients were calculated from three partitioned trajectories of 12 ns NPT simulations (each partition has 4 ns length), and the error bars were evaluated from the diffusion coefficients obtained from the three partitioned trajectories. Up to $373.15 \mathrm{~K}$, the diffusion coefficient increases with increasing temperature, whereas it decreases beyond $373.15 \mathrm{~K}$ due to the depletion of the water solvation. 


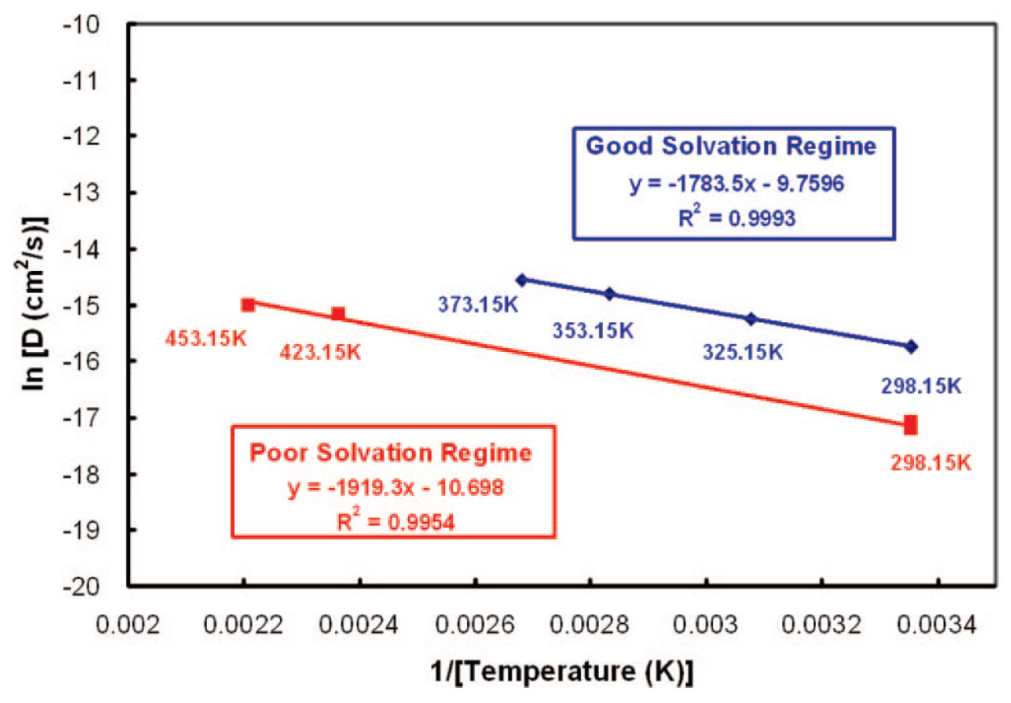

Figure 4.10: Arrhenius plots of diffusion coefficient from each solvation regime: the red squares are for the poor solvation regime and the blue diamond is for the good solvation regime. The solid black line is a linear fitting of each regime. This leads to $D_{300 K}=3.762 \times 10^{-8} \mathrm{~cm}^{2} / \mathrm{s}$ and $E_{a c t}=3.810$ $\mathrm{kcal} / \mathrm{mol}$ for the poor solvation regime and $D_{300 K}=1.512 \times 10^{-7} \mathrm{~cm}^{2} / \mathrm{s}$ and $E_{a c t}=3.540 \mathrm{kcal} / \mathrm{mol}$ for the good solvation regime, and the equation of each fitted line and $\mathrm{R} 2$ value are inset in the figure.

the internal space of zeolite remains hydrophobic. Below the pressure of $101.3250 \mathrm{kPa}$, the water uptake of the zeolite decreases monotonically with increasing temperature. From the pair correlation functions, we found that the tetrahedral water solvation structure for water molecule is suppressed by the hydrophobic pore surfaces. In contrast, the water solvation structure surrounding the sodium ion remains $\sim 5.0$. This difference is a direct consequence of the strong electrostatic interaction of the positively charged sodium ion with water molecules.

The MD trajectories indicate that the sodium ions are electrostatically bound to the aluminumdoped sites and the diffusion of the sodium ions proceeds via hopping mechanism among these aluminum-doped sites (Figure 4.8).

On the basis of the spontaneous water condensation process, we found the two regimes: a good solvation regime below $373.15 \mathrm{~K}$ and a poor solvation regime above $400 \mathrm{~K}$. The Arrhenius plot of each regime leads to an energy barrier of $3.540 \mathrm{kcal} / \mathrm{mol}$ for the diffusion of sodium ion for the good solvation regime and of $3.810 \mathrm{kcal} / \mathrm{mol}$ for the poor solvation regime. This leads to an estimate of $0.270 \mathrm{kcal} / \mathrm{mol}$ for the stabilization energy due to the solvation of sodium ion.

This study of the sodium diffusion through BEA zeolite shows that the ionic diffusion in the confined geometry depends on the degree of solvation by water which distinguishes it from bulk diffusion. As a next step, we are planning to directly investigate the proton diffusion through the aluminum-doped zeolite BEA system based on results of the current study. 


\subsection{Acknowledgments}

This research was supported in part by the Department of Energy (DE-FG02-05ER15716, William S. Millman). The facilities of the Materials and Process Simulation Center used for these studies were supported by DURIP-ARO, DURIP-ONR. 


\section{Bibliography}

[1] Zones, S. I.; Davis, M. E. Curr. Opin. Solid State Mater. Sci. 1996, 1, 107-117.

[2] Davis, M. E. Nature 2002, 417, 813-821.

[3] Truskett, T. M.; Debenedetti, P. G.; Torquato, S. J. Chem. Phys. 2001, 114, 2401-2418.

[4] Gallo, P.; Ricci, M. A.; Rovere, M. J. Chem. Phys. 2002, 116, 342-346.

[5] Giaya, A.; Thompson, R. W. J. Chem. Phys. 2002, 117, 3464-3475.

[6] Brovchenko, I.; Geiger, A.; Oleinikova, A. J. Chem. Phys. 2004, 120, 1958-1972.

[7] Maurin, G.; Bell, R. G.; Devautour, S.; Henn, F.; Giuntini, J. C. J. Phys. Chem. B 2004, 108, 3739-3745.

[8] Lee, Y.; Vogt, T.; Hriljac, J. A.; Parise, J. B.; Hanson, J. C.; Kim, S. J. Nature 2002, 420, 485-489.

[9] Demkov, A. A.; Sankey, O. F. Chem. Mater. 1996, 8, 1793-1806.

[10] Maurin, G.; Llewellyn, P.; Poyet, T.; Kuchta, B. J. Phys. Chem. B 2005, 109, 125-129.

[11] Calero, S.; Dubbeldam, D.; Krishna, R.; Smit, B.; Vlugt, T. J. H.; Denayer, J. F. M.; Martens, J. A.; Maesen, T. L. M. J. Am. Chem. Soc. 2004, 126, 11377-11386.

[12] Carrette, L.; Friedrich, K. A.; Stimming, U. ChemPhysChem 2000, 1, 162-193.

[13] Kreuer, K. D. J. Membr. Sci. 2001, 185, 29-39.

[14] Paddison, S. J. Annu. Rev. Mater. Res. 2003, 33, 289-319.

[15] Li, Q. F.; He, R. H.; Jensen, J. O.; Bjerrum, N. J. Chem. Mater. 2003, 15, 4896-4915.

[16] Mauritz, K. A.; Moore, R. B. Chem. Rev. 2004, 104, 4535-4585.

[17] Hickner, M. A.; Pivovar, B. S. Fuel Cells 2005, 5, 213-229. 
[18] Holmberg, B. A.; Hwang, S. J.; Davis, M. E.; Yan, Y. S. Microporous Mesoporous Mater. 2005, 80, 347-356.

[19] Burchart, E. D.; Verheij, V. A.; Vanbekkum, H.; Vandegraaf, B. Zeolites 1992, 12, 183-189.

[20] Levitt, M.; Hirshberg, M.; Sharon, R.; Laidig, K. E.; Daggett, V. J. Phys. Chem. B 1997, 101, 5051-5061.

[21] Rappe, A. K.; Goddard, W. A. J. Phys. Chem. 1991, 95, 3358-3363.

[22] Hockney, R. W.; Eastwood, J. W. Computer simulation using particles; McGraw-Hill: New York, 1981.

[23] Razmus, D. M.; Hall, C. K. AiChE J. 1991, 37, 769-779.

[24] Goodbody, S. J.; Watanabe, K.; Macgowan, D.; Walton, J.; Quirke, N. J. Chem. Soc., Faraday Trans. 1991, 87, 19511958.

[25] Accelrys Inc.: San Diego, CA, 1999.

[26] Allen, M. P.; Tildesley, D. J. Computer Simulation of Liquids; Oxford University Press: Oxford, U.K., 1987.

[27] Frenkel, D.; Smit, B. Understanding Molecular Simulations: From Algorithms to Applications; Academic Press: London, 2002.

[28] Plimpton, S. J. Comput. Phys. 1995, 117, 1-19.

[29] Plimpton, S. J.; Pollock, R.; Stevens, M. Minneapolis, MN, 1997.

[30] Swope, W. C.; Andersen, H. C.; Berens, P. H.; Wilson, K. R. J. Chem. Phys. 1982, 76, 637-649.

[31] Baerlocher, C.; McCusker, L. B. Database of zeolite Structures: http://www.izastructure.org/databases/.

[32] Di Lella, A.; Desbiens, N.; Boutin, A.; Demachy, I.; Ungerer, P.; Bellat, J. P.; Fuchs, A. H. Phys. Chem. Chem. Phys. 2006, 8, 5396-5406.

[33] Desbiens, N.; Demachy, I.; Fuchs, A. H.; Kirsch-Rodeschini, H.; Soulard, M.; Patarin, J. Angew. Chem., Int. Ed. 2005, 44, 5310-5313.

[34] Desbiens, N.; Boutin, A.; Demachy, I. J. Phys. Chem. B 2005, 109, 24071-24076.

[35] Hummer, G.; Rasaiah, J. C.; Noworyta, J. P. Nature 2001, 414, 188-190.

[36] Gorbach, A.; Stegmaier, M.; Eigenberger, G. Ads.-J. Int. Ads. Soc. 2004, 10, 29-46. 
[37] Soper, A. K.; Phillips, M. G. Chem. Phys. 1986, 107, 47-60.

[38] Jang, S. S.; Goddard, W. A., III J. Phys. Chem. C 2007, 111, 2759-2769.

[39] Jang, S. S.; Lin, S. T.; Cagin, T.; Molinero, V.; Goddard, W. A., III J. Phys. Chem. B 2005, 109, 10154-10167.

[40] Jang, S. S.; Molinero, V.; Cagin, T.; Goddard, W. A., III J. Phys. Chem. B 2004, 108, 31493157.

[41] Vaudry, F.; DiRenzo, F.; Espiau, P.; Fajula, F.; Schulz, P. Zeolites 1997, 19, 253-258.

[42] Keffer, D.; McCormick, A. V.; Davis, H. T. Mol. Phys. 1996, 87, 367-387.

[43] Faux, D. A.; Smith, W.; Forester, T. R. J. Phys. Chem. B 1997, 101, 1762-1768.

[44] Faux, D. A. J. Phys. Chem. B 1998, 102, 10658-10662.

[45] Faux, D. A. J. Phys. Chem. B 1999, 103, 7803-7808.

[46] Franke, M. E.; Simon, U. Phys. Status Solidi B: Basic Res. 2000, 218, 287-290.

[47] Kim, K. K.; Mundy, J. N.; Chen, W. K. J. Phys. Chem. Solids 1979, 40, 743-755.

[48] Whitting, Ms; Huggins, R. A. J. Chem. Phys. 1971, 54, 414. 


\section{Chapter 5}

\section{Experimental and Theoretical Investigation into the Correlation between Mass and Ion Mobility for Choline and Other Ammonium Cations in $\mathrm{N}_{2}$}

Reproduced with permission from Kim, H; Kim, H. I.; Johnson, P. V.; Beegle, L. W.; Beauchamp, J. L.; Goddard, W. A.; Kanik, I. Anal. Chem. 2008, 80, 1928. Copyright 2008 American Chemical Society.

\subsection{Abstract}

A number of tertiary amine and quaternary ammonium cations spanning a mass range of $60-146 \mathrm{amu}$ (trimethylamine, tetramethylammonium, trimethylethylammonium, $N, N$-dimethylaminoethanol, choline, $N, N$-dimethylglycine, betaine, acetylcholine, (3-carboxypropyl)trimethylammonium) were investigated using electrospray ionization ion mobility spectrometry. Measured ion mobilities demonstrate a high correlation between mass and mobility in $\mathrm{N}_{2}$. In addition, identical mobilities within experimental uncertainties are observed for structurally dissimilar ions with similar ion masses. For example, dimethylethylammonium (88 amu) cations and protonated $N, N$-dimethylaminoethanol cations (90 amu) show identical mobilities $\left(1.93 \mathrm{~cm}^{2} \mathrm{~V}^{-1} \mathrm{~s}^{-1}\right)$ though $N, N$-dimethylaminoethanol contains a hydroxyl functional group while dimethylethylammonium only contains alkyl groups. Computational analysis was performed using the modified trajectory (TJ) method with nonspherical $\mathrm{N}_{2}$ molecules as the drift gas. The sensitivity of the ammonium cation collision cross-sections to the details of the ion-neutral interactions was investigated and compared to other classes of organic molecules (carboxylic acids and abiotic amino acids). The specific charge distribution of the 
molecular ions in the investigated mass range has an insignificant affect on the collision cross-section.

\subsection{Introduction}

The development of soft ionization methods such as electrospray ionization (ESI) [1] have expanded the application of ion mobility spectrometry (IMS) $[2,3]$ to structural investigations of nonvolatile biomolecules in the gas phase [4]. ESI allows soft sampling by transferring intact ions directly from the solution phase to the gas phase. Using this distinctive advantage of ESI, the shapes and sizes of various biomolecular ions from monomeric molecules to macrosize protein complexes have been investigated. The combination of ESI and IMS has facilitated conformational studies of macroions including clusters (oligomers) $[5,6,7]$, peptides [8, 9], and proteins $[10,11,12]$. In addition, ion mobilities of organic molecules such as amino acids [13, 14], carboxylic acids [15], and dinucleotides [16], as well as other organic molecules $[17,18]$, have been reported.

To provide a firm foundation for studies of the shapes of complex organic molecular ions using IMS, many research groups have endeavored to develop theoretical models to predict ion mobilities and related cross-sections of gas-phase molecular ions. Griffin et al. [19] have shown that mass and mobility are only correlated on the order of $20 \%$ within a collection of structurally unrelated compounds spanning a mass range of $\sim 70-500 \mathrm{amu}$. The correlations are improved up to $2 \%$ when only structurally related compounds are considered. Karpas and co-workers have established models to predict the mobility for a number of compound classifications including acetyls, aromatic amines, and aliphatic amines drifting in $\mathrm{He}, \mathrm{N}_{2}$, air, $\mathrm{Ar}, \mathrm{CO}_{2}$, and $\mathrm{SF}_{6}[20,21]$. Our laboratory has applied a $12-4$ potential model in studies of amino acids and carboxylic acids drifting in $\mathrm{N}_{2}$ and $\mathrm{CO}_{2}[14,15]$. Recently, Steiner et al. have reported predictions of mobilities for a series of different classes of amines (primary, secondary, tertiary) in various drift gases, such as He, Ne, Ar, $\mathrm{N}_{2}$, and $\mathrm{CO}_{2}$, using several theoretical models (rigid-sphere, polarization-limit, $12-6-4$, and $12-4$ potential model) [22].

Computational modeling related to interpretation of IMS data has been developed by several groups. Efforts toward theoretical ion mobility predictions using computational methods face difficulties associated with complicated collision integrals and the design of functions to accurately describe the ion-neutral interaction potential. Bowers and co-workers have proposed a project approximation method, which is based on a hard-sphere description of the interaction potential [23]. The trajectory (TJ) method, which has been proposed by Jarrold and co-workers, adopts more realistic soft-core interactions [24].

Ion mobility constants $(K)$ can be derived from the collision cross-section using the equation [25]

$$
K=\frac{(18 \pi)^{1 / 2}}{16} \frac{1}{\mu^{1 / 2}} \frac{z e}{\left(k_{B} T\right)^{1 / 2} \Omega_{D}} \frac{1}{N}
$$


where $\mu$ is reduced mass, $N$ is the number density of the neutral gas molecule, and $z$ is the charge of the ion. The collision cross-section, $\Omega_{D}$, is given by [24]

$$
\Omega_{D}=\frac{1}{8 \pi^{2}} \int_{0}^{2 \pi} d \theta \int_{0}^{\pi} d \phi \int_{0}^{2 \pi} d \gamma \frac{\pi}{8}\left(\frac{\mu}{k_{B} T}\right)^{3} \int_{0}^{\infty} d g e^{-\mu / 2 k_{B} T} g^{5} \int_{0}^{\infty} d b 2 b(1-\cos \chi(\theta, \phi, \gamma, g, b))
$$

and $\theta, \phi$, and $\gamma$ are the three-dimensional collision angles, $g$ is the relative velocity, and $b$ is the impact parameter. Because the scattering angle $\chi(\theta, \phi, \gamma, g, b)$ depends on the pairwise potential between the ion and neutral gas molecules, the accuracy of computed cross-section values is determined by the quality of the interaction potential model. The potential employed in the TJ method [24] for a He drift gas is given by

$$
\Phi(\theta, \phi, \gamma, b, r)=4 \epsilon \sum_{i}^{n}\left[\left(\frac{\sigma}{r_{i}}\right)^{12}-\left(\frac{\sigma}{r_{i}}\right)^{6}\right]-\frac{\alpha}{2}\left(\frac{z e}{n}\right)^{2}\left[\left(\sum_{i}^{n} \frac{x_{i}}{r_{i}^{3}}\right)^{2}+\left(\sum_{i}^{n} \frac{y_{i}}{r_{i}^{3}}\right)^{2}+\left(\sum_{i}^{n} \frac{z_{i}}{r_{i}^{3}}\right)^{2}\right] .
$$

The first term is a sum over short-range van der Waals interactions, and the second term represents long-range ion-induced dipole interactions. In the expression, $\epsilon$ is the depth of the potential well, $\sigma$ is the value of distance $(r)$ between the centers of mass of the each atom in the ion and neutral gas molecule at the potential minimum, and $R$ is the neutral polarizability. The coordinates, $r_{i}, x_{i}$, $y_{i}$, and $z_{i}$, are defined by the relative positions of the atoms with respect to the neutral. Utilizing the given ion-neutral interaction potential functions, the integrals in Equation 5.2 can be processed numerically. Monte Carlo integration schemes are used for the integration over $\theta, \phi, \gamma$, and b. The numerical integration over $g$ is performed using a combination of the Runge-Kutta-Gill integration method and the Adams-Moulton predictor corrector integration method.

Choline is a precursor for phosphatidylcholine, sphingomyelin, and other important biological molecules [26]. Further, it is a component of cell membrane lipids in biological systems, and it plays an important role in their repair. Choline can be oxidized to betaine, which is readily demethylated to yield $N, N$-dimethylglycine [26]. Decomposition of choline yields trimethylamine and dimethylamine [27]. Searching for lipids and their components (i.e., choline) may be a valuable strategy in the search for evidence of extinct or extant life elsewhere in the cosmos. Under the high oxidizing conditions and significant ultraviolet flux found on the surface of Mars, one would expect decomposition products of lipids to include various alkylamines [28].

In the present study, mobilities have been measured for a number of quaternary and tertiary ammonium cations related to choline and its derivates drifting in $\mathrm{N}_{2}$. Of particular interest was the possible dependence of mass-mobility correlations with the heavy atom $(\mathrm{C}, \mathrm{N}, \mathrm{O})$ complements present in the molecular ion, comparing, for example, alkylated ammonium ions to abiotic amino acids (betaine and $N, N$-dimethylglycine). A modified TJ method for the ion-neutral interaction, 
to account for the potential associated with the nonspherical drift gas $\mathrm{N}_{2}$, has been applied to predict cross-sections of these polyatomic ammonium cations and to test the sensitivity of collision cross-section to details of the ion-neutral interaction. Comparisons of the results from the ammonium cations to other classes of organic molecules (carboxylic acids and abiotic amino acids) are presented. The origin of the observed correlation between mass and mobility of ammonium cations is discussed.

\subsection{Experimental Section}

\subsubsection{Chemicals and Reagents}

All the compounds studied in this work were purchased from Sigma Aldrich (St. Louis, MO) and were used without further purification. All solvents (water, methanol, acetic acid) were HPLC grade and were purchased from EMD Chemicals Inc. (Gibbstown, NJ). Quaternary ammonium samples were prepared by dissolving known quantities of ammonium ions in a solvent consisting of $50 \%$ water and $50 \%$ methanol by volume to give sample concentrations in the range of $100 \mu \mathrm{M}$. Tertiary amine samples were prepared as $300 \mu \mathrm{M}$ in a solvent of 50:50 water and methanol with $1 \%$ acetic acid by volume.

\subsubsection{Electrospray Ionization Ion Mobility Spectrometer}

The ESI-IMS instrument and the data acquisition system used in this investigation were based on designs previously described by Hill and co-workers [17, 29] and have been described in detail by Johnson et al. [14]. The drift length of the ion mobility spectrometer was $13.65 \mathrm{~cm}$ and was operated in the positive mode. A drift voltage of $3988 \mathrm{~V}$, corresponding to electric field strength of $292 \mathrm{~V} / \mathrm{cm}$, was employed. All measurements were made at local atmospheric pressure $(\sim 730$ Torr $)$ while a counterflow of the preheated drift gas was introduced at the detection end of the drift region at a flow rate of $\sim 800 \mathrm{~mL} / \mathrm{min}$. The sample solution was delivered by an Eldex Micropro liquid chromatography pump at a flow rate of $3 \mu \mathrm{L} / \mathrm{min}$ into a stainless steel electrospray needle, which was held at a potential $3-4 \mathrm{kV}$ above the entrance to the desolvation region of the spectrometer. The gap between the electrospray needle and the entrance electrode was $\sim 2 \mathrm{~cm}$.

Ions were introduced into the drift region through the ion gate in 0.2-ms pulses. Signals collected at the Faraday cup were amplified by a factor of $10^{9}$ (Stanford Research Systems model SR570 low-noise current preamplifier) and recorded as a function of drift time in 0.02-ms-wide channels. Typically, 1000 individual $0-25$-ms scans were averaged to produce the final spectra used in the analysis. Resolution of the instrument was found to be $\sim 0.43 \mathrm{~ms}$ full width at half-maximum (FWHM) with drift times in the range 12-17 ms for the ions studied and the parameters employed in these experiments. 
Throughout this work, it was assumed that ESI of the prepared samples resulted in singly charged ammonium cations. The assumption was confirmed by ESI mass spectrometric analysis using a Finnigan LCQ Deca XP ion trap mass spectrometer. The mass spectra of all nine samples in the present study show singly charged monomeric molecular cations as the major ionic species. Since the experiments were conducted with the drift cell at $473 \mathrm{~K}$, it was further assumed that there was no significant water cluster formation based on previous IMS-MS studies [18, 30].

Reduced ion mobilities, $K_{0}$, were determined from the recorded spectra and the experimental parameters according to the usual relation,

$$
K_{0}=\left(\frac{273 \mathrm{~K}}{T}\right)\left(\frac{P}{760 \text { Torr }}\right) \frac{D^{2}}{V t},
$$

where $V$ is the voltage drop across the drift region, $D$ is the drift length, $t$ is the drift time, $P$ is the pressure, and $T$ is the temperature. With the above parameters expressed in units of $\mathrm{V}, \mathrm{cm}, \mathrm{s}$, Torr, and K, respectively, Equation 5.4 gave the reduced mobility in the typical units of $\mathrm{cm}^{2} \mathrm{~V}^{-1}$ $\mathrm{s}^{-1}$. The experimental uncertainties of the determined $K_{0}$ values are estimated to be $\sim 3 \%$ based on the half width at half-maximum (HWHM) of each drift time peak in the averaged ion mobility spectra.

\subsubsection{Computational Modeling}

More than 500 possible molecular conformations were investigated through dihedral angles of $-180^{\circ}$ to $180^{\circ}$ at the PM5 level using CAChe 6.1 .12 (Fujitsu, Beaverton, OR). Then, the lowest-energy structures were determined using density functional theory (DFT) with a number of candidate lowenergy structures from the previous PM5 calculations. DFT calculations were performed using Jaguar 6.0 (Schrödinger, Inc., Portland, OR) utilizing the Becke three-parameter functional (B3) [31] combined with the correlation functional of Lee, Yang, and Parr (LYP) [32], using the 6-31G** basis set [33]. The optimized structures of ammonium cations investigated in the present study are shown in Figure 5.2.

The TJ method [24], originally developed by Jarrold and coworkers, was modified to describe the interaction between ions and an $\mathrm{N}_{2}$ drift gas and expand the applicability of the TJ method beyond cases of ions drifting in He. As shown in Equation 5.3, the potential used in the original TJ method consists of two terms representing van der Waals and ion-induced dipole interactions, which are characterized by the Lennard-Jones parameters $(\epsilon, \sigma)$ and the neutral polarizability $(\alpha)$, respectively. We set the polarizability of $\mathrm{N}_{2}$ at the experimentally determined value [34] of $1.710 \times 10^{-24} \mathrm{~cm}^{2}$ and took the Lennard-Jones parameters described in the universal force field [35], which is a general purpose force field optimized for all the elements in the periodic table. Due to the linear geometry of $\mathrm{N}_{2}$, two more consequences should be additionally taken into account; the ion-quadrupole interaction 
and the orientation of the molecule. We mimic the quadrupole moment of $\mathrm{N}_{2},(-4.65 \pm 0.08) \times 10^{-40}$ $\mathrm{C} \mathrm{cm}^{2}[36]$, by displacing charges by negative $q(0.4825 e)$ to each nitrogen atom and one positive $2 q$ at the center of the nitrogen molecule. Hence, the ion-quadrupole potential can be expressed with simple summations of partial charges as follows:

$$
\Phi_{I Q}=\sum_{j=1}^{3} \sum_{i}^{n} \frac{z_{i} z_{j} e^{2}}{r_{i j}}
$$

where indexes $i$ and $j$ denote the atoms of the ion, three points of $\mathrm{N}_{2} \cdot j=1$ and 3 indicate the two nitrogen atoms, and $j=2$ indicates the center of mass position of $\mathrm{N}_{2}$.

To consider the orientation of the nitrogen molecule rigorously, all possible trajectories with varying molecular orientations were taken into account. It has been widely accepted that the ion field does not exclusively quench the rotational angular momentum of the neutral molecule and only partial locking occurs during the collision process [37, 38]. Thus, we assumed that the interaction potential averaged over the rotational degree of freedom generates an appropriated average impact parameter [39]. The calculated rotation time of a $\mathrm{N}_{2}$ molecule $(\sim 620 \mathrm{~ns})$ implies that approximately three molecular rotations occur during a collision between an ion and $\mathrm{N}_{2}$ taking place $(\sim 2 \mathrm{ps})$. The orientations of $\mathrm{N}_{2}$ are sampled along with $x, y$, and $z$ axes to represent all the three-dimensional rotational space. Then the orientation averaged interaction potential is evaluated using Boltzmann weighting. Using these different weights, the orientation averaged interaction potential is evaluated, and this potential is used to compute the collision cross-section.

For the calculations of collision cross-section of ions, it is assumed that the DFT optimized structures are rigid. To ensure that the assumption is valid for the ammonium cations investigated in the present study, the collision cross-sections of two extreme conformations for the largest two ionic molecules, acetylcholine and (3-carboxypropyl)trimethylammonium, are estimated. The DFT calculated electronic energies reveal that the extended structures of both acetylcholine and (3-carboxypropyl)trimethylammonium are unstable by 4.24 and $0.547 \mathrm{kcal} / \mathrm{mol}$, respectively, compared to cyclic structures shown in Figure 5.2. The maximum difference between two conformations

of (3-carboxypropyl)-trimethylammonium is calculated as $\sim 7 \AA^{2}$, which we can set as a maximum error bound originating from the structural uncertainty.

\subsection{Results}

\subsubsection{Mass-Mobility Correlation of Ammonium Cations}

IMS spectra were obtained as described above. The drift times of the ammonium cations were determined from the location of the peak maximums. Figure 5.1 shows example spectra taken with pure 


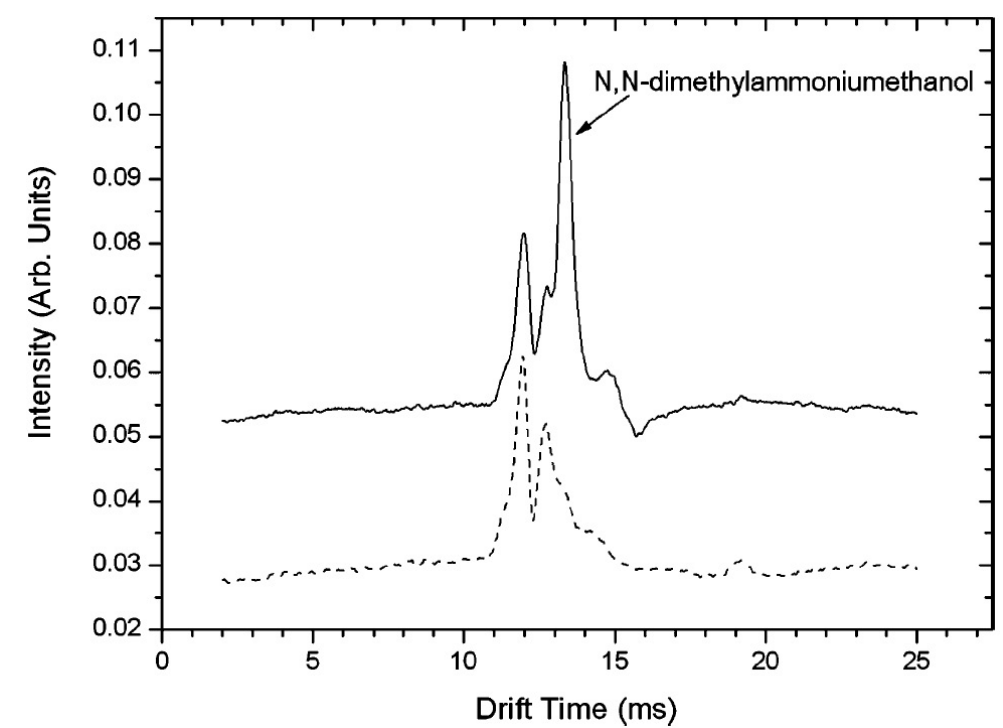

Figure 5.1: Examples of the ion mobility spectra taken in this study. Shown are two spectra taken in 730 Torr $\mathrm{N}_{2}$. The electric field strength and the temperature of the drift tube were 292 $\mathrm{V} / \mathrm{cm}$ and $473 \mathrm{~K}$, respectively. The dash curve is a spectrum taken with pure solvent being introduced to the electrospray needle while the solid curve is a spectrum of solvent and $300 \mu \mathrm{M} N, N$ dimethylammoniumethanol. The two spectra were smoothed (10 point adjacent averaging) and shifted in intensity by an additive constant to avoid overlap. The $N, N$-dimethylammoniumethanol feature is indicated in the figure. The unlabeled features correspond to ionized solvent (water, methanol, acetic acid) and atmospheric constituents ionized through proton transfer (due to the open nature of the ESI-IMS instrument). 
solvent being introduced to the electrospray needle and with $300 \mu \mathrm{M} N, N$-dimethylammoniumethanol dissolved in the solvent. These spectra are characteristic of those considered in this work. Measured drift times, reduced ion mobilities (in $\mathrm{N}_{2}$ drift gas), and determined $\Omega_{D}$ for the nine ammonium cations chosen for this study are listed in Table 5.1 along with their respective molecular weights. The $12-4$ potential model, which has proven satisfactory to model experimental data $[14,15,20,21,22]$ has been used for the analysis of the experimentally determined mobilities of ammonium cations. The potential is expressed as

$$
\Phi(r)=\frac{\epsilon}{2}\left\{\left(\frac{\sigma-a}{r-a}\right)^{12}-3\left(\frac{\sigma-a}{r-a}\right)^{4}\right\}
$$

where $\epsilon, r$, and $\sigma$ are defined above and the parameter $a$ is the location of center charge from the center of mass in the ion. Rearrangement of Equation 5.6, along with the substitution of the appropriate constants, yields

$$
K_{0}^{-1}=\left(1.697 \times 10^{-4}\right)(\mu T)^{1 / 2} \sigma^{2} \Omega^{(1,1) *},
$$

which gives the reduced ion mobility in terms units of $\mathrm{cm}^{2} \mathrm{~V}^{-1} \mathrm{~s}^{-1} \cdot \Omega^{(1,1) *}$ is the dimensionless collision integral, where $\Omega_{D}=\pi \sigma^{2} \Omega^{(1,1) *}$. Derivation of Equation 5.7 from Equation 5.6 is well described by Johnson et al. [14]. Equation 5.7 was fit to the data set of ammonium ion mobilities in $\mathrm{N}_{2}$ using a nonlinear least-squares fitting procedure [14]. The plot of $K_{0}^{-1}$ versus ion mass for ammonium cations drifting in $\mathrm{N}_{2}$ is shown in Figure 5.2 along with the best fit to the data. As seen in Figure 5.2, all nine ammonium cations investigated in the present study exhibit a good correlation $\left(R^{2}=0.99\right)$ between mass and mobility of ion. In particular, the two different classes of ammonium cations (tertiary and quaternary) investigated in this study exhibit a common massmobility correlation. Further, the heteroatomic complements of the molecular ions do not impact the mass-mobility correlation.

\subsubsection{Tertiary and Quaternary Ammonium Cations with Similar Molec- ular Weights}

Two sets of cations, which have similar molecular weights but different structures, were chosen to investigate the influence of the composition and structural details of the ion on the mobility. The molecular weights of trimethylethylammonium and $N, N$-dimethylammnoiumethanol are 88 and $90 \mathrm{amu}$, respectively. There is a significant structural difference between these two ions in addition to variation in the degree of alkylation to the ammonium groups. Protonated $N, N$ dimethylammoniumethanol possesses a hydroxyl group at the ethyl group while trimethylethylammonium possesses only alkyl groups. The molecular weights of choline and $N, N$-dimethylglycine 

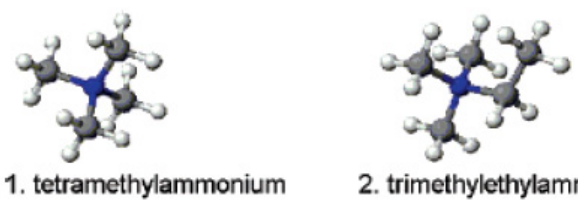

2. trimethylethylammonium
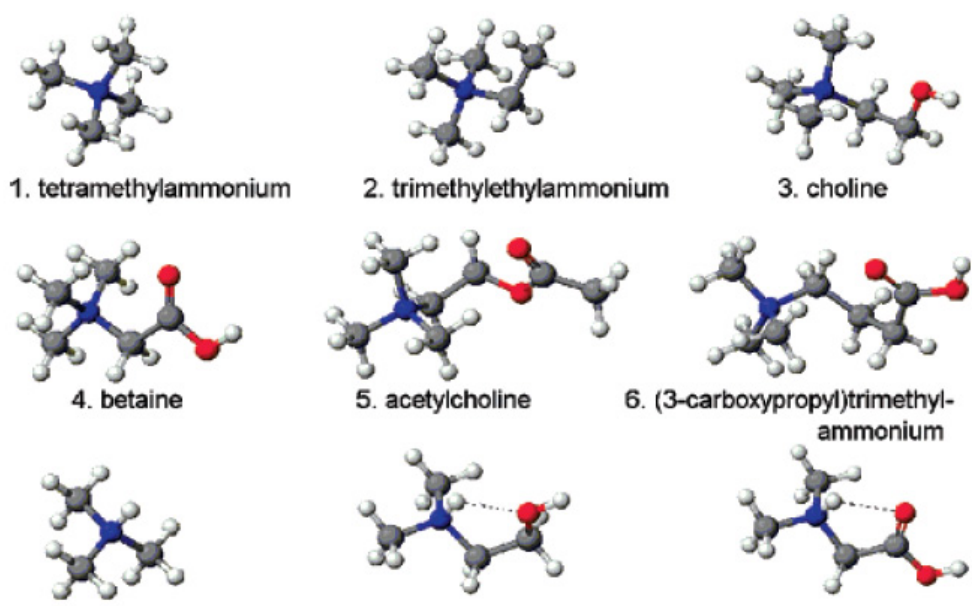

5. acetylcholine

6. (3-carboxypropyl)trimethyl-

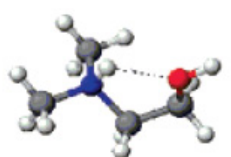
ammonium

$\begin{array}{lll}\text { a. trimethylammonium } & \text { b. } \mathrm{N}, \mathrm{N} \text {-dimethylammoniumethanol } & \text { c. } \mathrm{N}, \mathrm{N} \text {-dimethylglycine }\end{array}$

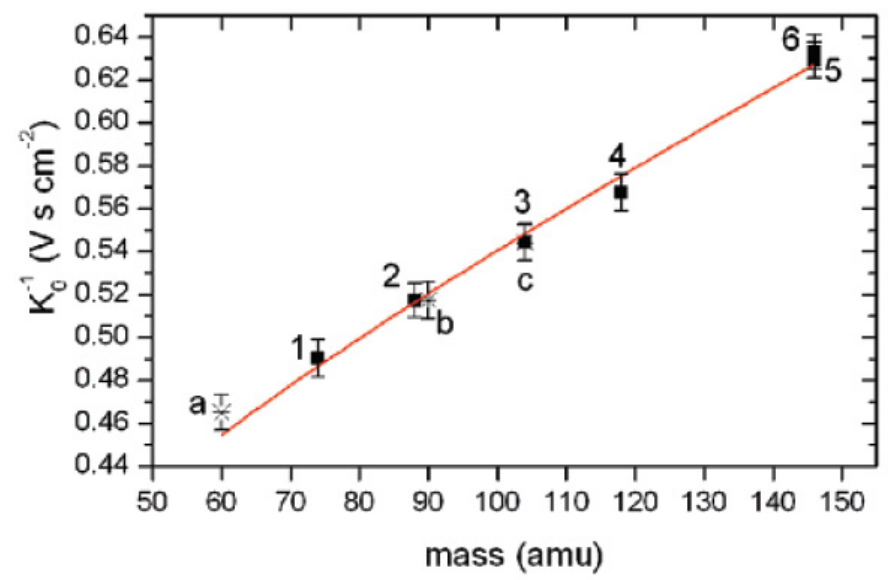

Figure 5.2: Plot of $K_{0}^{-1}$ for $3^{\circ}$ and $4^{\circ}$ ammonium cations drifting in $\mathrm{N}_{2}$ versus ion mass. Experimentally determined data for $3^{\circ}$ ammonium and $4^{\circ}$ ammonium cations are shown as asterisks and solid squares, respectively. The solid line is the fit of the $12-4$ potential model to the ammonium cation data set. DFT optimized structure of each numerically or alphabetically labeled ion is shown above. Optimized geometries are obtained at B3LYP/6-31G** level. The hydrogen bonds are indicated with dashed lines. 
Table 5.1: Drift Times, Reduced Mobilities, and Collision Cross-Sections of Ammonium Cations in $\mathrm{N}_{2}$ Drift Gas

\begin{tabular}{lcccc}
\hline \hline ammonium cation & ${ }^{a} \mathrm{MW}$ & ${ }^{b} \mathrm{DT}$ & ${ }^{c} K_{0}$ & ${ }^{d} \Omega_{D}$ \\
\hline trimethylammonium & 60 & 12.1 & 2.15 & 91.2 \\
tetramethylammonium & 74 & 12.7 & 2.04 & 95.3 \\
trimethylethylammonium & 88 & 13.4 & 1.93 & 102.2 \\
$N, N$-dimethylammoniumethanol & 90 & 13.4 & 1.93 & 100.9 \\
choline & 104 & 14.1 & 1.84 & 104.5 \\
$N, N$-dimethylglycine & 104 & 14.1 & 1.84 & 102.3 \\
betaine & 118 & 14.7 & 1.76 & 105.3 \\
acetylcholine & 146 & 16.3 & 1.59 & 118.5 \\
(3-carboxypropyl)trimethylammonium & 146 & 16.4 & 1.58 & 115.9 \\
\hline \hline
\end{tabular}

${ }^{a}$ Molecular weight (amu). ${ }^{b}$ Drift time $(\mathrm{ms}) .{ }^{c}$ Reduced mobility $\left(\mathrm{cm}^{2} \mathrm{~V}^{-1} \mathrm{~s}^{-1}\right) .{ }^{d}$ Collision crosssection $\left(\AA^{2}\right)$.

cation are both 104 amu. Protonated $N, N$-dimethylglycine cations contain a carboxyl group while choline possesses a hydroxyl group. Experimentally determined mobility values of trimethylethylammonium and $N, N$-dimethylammoniumethanol are identical at $1.93 \mathrm{~cm}^{2} \mathrm{~V}^{-1} \mathrm{~s}^{-1}$. Mobilities of both choline and $N, N$-dimethylglycine cation are measured as $1.84 \mathrm{~cm}^{2} \mathrm{~V}^{-1} \mathrm{~s}^{-1}$. It is inferred that the contribution of the oxygen atom to the mobility (ion-neutral ion-neutral interaction) is not significantly different from that of a methylene group in the investigated ammonium cations.

\subsubsection{Functional Group Isomers of Ammonium Cations}

Two functional group isomers, acetylcholine and (3-carboxypropyl)-trimethylammonium cation, were examined to study the influence of the location of oxygen atoms on the molecular ion's mobility. As seen in Figure 5.2, acetylcholine and (3-carboxypropyl)trimethylammonium are not distinguishable based on their mobilities.

\subsubsection{Collision Cross-Sections of Ions in $\mathrm{N}_{2}$ via the Trajectory Method}

Theoretical $\Omega_{D}$ of the ammonium cations investigated in this study were evaluated using the modified TJ method. Prior to application of the modified TJ method to the ammonium cations, we tested the model on previously published experimental data. Figure 5.3a shows the plot of experimentally determined $\Omega_{D}$ of carboxylic acid anions [15] and abiotic amino acid cations [14] in $\mathrm{N}_{2}$ versus those determined theoretically using the modified TJ method following the procedure described in the Experimental Section. Theoretical $\Omega_{D}$ of both carboxylic acid anions and abiotic amino acid cations exhibit good agreement with experimental values. The agreement is within $5 \%$ in the worst-case deviation with less than $2 \%$ deviation on average. Figure 5.3b shows the plot of $\Omega_{D}$ of ammonium cations obtained experimentally versus theoretical collision cross-sections calculated 

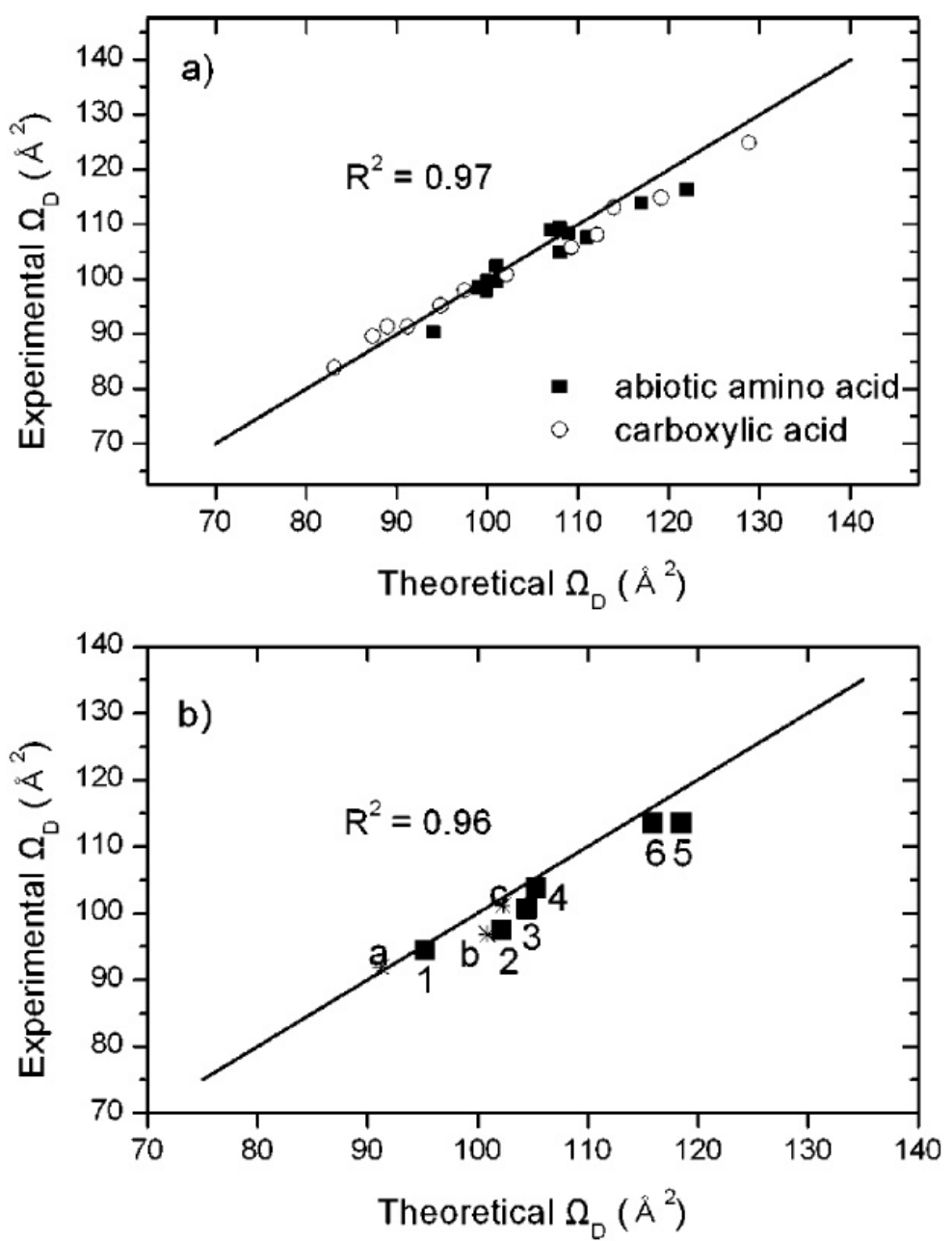

Figure 5.3: (a) Plot of experimentally determined collision cross-sections $\left(\Omega_{D}\right)$ of abiotic amino acid cations [14] and carboxylic acid anions [15] in $\mathrm{N}_{2}$ versus theoretically determined $\Omega_{D}$ using the modified TJ method for $\mathrm{N}_{2}$ drift gas. Abiotic amino acid cation data are shown as solid squares and carboxylic acid anion data are shown as empty circles. The solid line is $y=x$. (b) Plot of experimentally determined collision cross-sections $\left(\Omega_{D}\right)$ of $3^{\circ}$ and $4^{\circ}$ ammonium cations in $\mathrm{N}_{2}$ versus theoretically determined $\Omega_{D}$ using the modified TJ method for $\mathrm{N}_{2}$ drift gas. $3^{\circ}$ ammonium cation data are shown as asterisks and $4^{\circ}$ ammonium cation data are shown as solid squares. Each ion is labeled with the appropriate identifying number and alphabet shown in Figure 5.1. The solid line is $y=x$. 
using the modified TJ method. The worst observed deviation of the model from the experimental cross-sections is $5 \%$ with an average deviation of $2.5 \%$.

\subsection{Discussion}

\subsubsection{Classical Ion-Neutral Collision Model}

The cross-section includes the information regarding the ion-neutral interaction. An ion and a neutral interact through the long-range ion-induced dipole potential, which is given by

$$
\Phi_{I I D}=-\frac{(z e)^{2} \alpha}{2 r^{4}}
$$

where $z, R$, and $r$ are defined above. The effective potential, $\Phi_{e f f}(r)$, is expressed as $\Phi_{I I D}+L^{2} / 2 \mu r^{2}$, where $L$ is angular momentum of the collision partners about the center of mass of the combined system. The critical impact parameter $b^{*}=\left(2 \alpha e^{2} / \mathrm{KE}\right)^{1 / 4}$ is derived by setting $\mathrm{KE}$ equal to the maximum effective potential, $\Phi_{e f f}^{*}(r)$, which is given by $1 / 2(\mathrm{KE})^{2} b^{4} / \alpha e^{2}$, where $\mathrm{KE}$ is the relative kinetic energy. Then the Langevin capture cross-section is

$$
\Omega_{L}=\pi\left(b^{*}\right)^{2}=\pi \sqrt{\frac{2 \alpha e^{2}}{\mathrm{KE}}} .
$$

When the hard-sphere collision radius, $R_{c}$, is greater than $b^{*}$, the Langevin model is no longer appropriate and collisions are dominated by large angle deflections appropriate for a hard-sphere model. In this case, momentum transfer is no longer dominated by long-range interactions. In order to assess the ion-neutral collision under our experimental conditions, $b^{*}$ and $\Omega_{L}$ are evaluated from the mean relative kinetic energies. The evaluated $\Omega_{L}$ and $b^{*}$ are then compared to the experimental $\Omega_{D}$ and $R_{c}$ (Table 5.2). The hard-sphere collision radius $R_{c}$ is determined from the experimental $\Omega_{D}$ by equating it to $\pi R_{c}^{2}$. Experimental mean relative kinetic energies can be determined from the Wannier energy formula,

$$
\mathrm{KE}=\frac{1}{2} \mu g^{2}=\frac{3}{2} k_{B} T+\frac{1}{2} M v_{d}^{2}
$$

where $M$ is mass of drift gas molecule and $v_{d}$ is drift velocity of ion [40]. Under the current experimental conditions described in the Experimental Section, $b^{*}$ is calculated on the order of $5 \AA$. Comparison with $R_{c}$ shows that $b^{*}$ in our system is on the same order, i.e., less than $1 \AA$ smaller (Table 5.2). It is therefore inferred that the group of molecules studied here are on the borderline between being dominated by long-range versus short-range interactions, favoring some orbiting at lower collision energies, which would then determine the cross-section for momentum transfer and hence the mobility. 


\subsubsection{Computational Trajectory Method}

Ammonium cations investigated in this study exhibit a correlation between mass and mobility (Figure 5.2). In order to understand and estimate the effect of the each component of the ion-neutral interaction potential in terms of the observed mass-mobility correlation in our experimental system, theoretical calculations were performed using the modified TJ method. The collision cross-sections $\left(\Omega_{D}\right)$ were evaluated using molecular ions with restricted interaction potentials and artificial charge distributions. Comparisons of the $\Omega_{D}$ of tertiary $\left(3^{\circ}\right)$ and quaternary $\left(4^{\circ}\right)$ ammonium cations, abiotic amino acid cations, and carboxylic acid anions, which are calculated with different interaction potentials, are shown in Figures 5.4 and 5.5.

\subsubsection{Ion-Quadrupole Potential}

In order to understand the role of the ion-quadrupole interaction in ion-neutral interactions, the $\Omega_{D}$ are computed without ion-quadrupole interactions. The presence of the quadrupole moment elevates the $\Omega_{D}$ by $2.8 \%$ for the ammonium cations, $2.7 \%$ for the abiotic amino acid cations, and $4.2 \%$ for carboxylic acid anions (Figure 5.4a). Overall, it is observed that the addition of the ion-quadrupole potential to the model for ion- $\mathrm{N}_{2}$ interaction improves the agreement between experimental and theoretical $\Omega_{D}$ values. Previously, Su and Bowers reported quadrupole effects for molecules with high quadrupole moments using the average quadrupole orientation theory [41]. They demonstrated the significance of quadrupole effects, especially in the case when the ionic charge and quadrupole moment have the same polarity [41]. In analogy, a larger quadrupole effect is observed in carboxylic acid anions versus ammonium and abiotic amino acid cations, since nitrogen has a negative quadrupole moment. During the collision process, therefore, the change of a favorable orientation induced by the total ionic charge influences the collision cross-sections via ion-quadrupole interaction. This causes the observed difference of the $\mathrm{N}_{2}$ drift gas in ion-neutral interactions compared to spherical drift gas (i.e., He).

\subsubsection{Ion-Induced Dipole Potential}

In order to understand the effect of the long-range ion-induced dipole interactions between ions and neutral $\mathrm{N}_{2}$ molecules, theoretical collision cross-section with the van der Waals and ion-induced dipole potential $\left(\Omega_{D, V D W+I I D}\right)$ of molecular ions are compared to collision cross-sections computed after assigning the total charge of the ionic molecule as neutral $\left(\Omega_{D, V D W}\right)$. The calculated $\Omega_{D, V D W}$ with the van der Waals-only potential are $\sim 8-23 \%$ smaller than the calculated $\Omega_{D, V D W+I I D}$. The observed difference is attributed mainly to the lack of long-range interactions. Figure 5.4b shows plots of theoretically determined $\Omega_{D, V D W}$ over the theoretical $\Omega_{D, V D W+I I D}$ of $3^{\circ}$ and $4^{\circ}$ ammonium cations, abiotic amino acid cations, and carboxylic acid anions in $\mathrm{N}_{2}$ versus ion mass. The agreement 

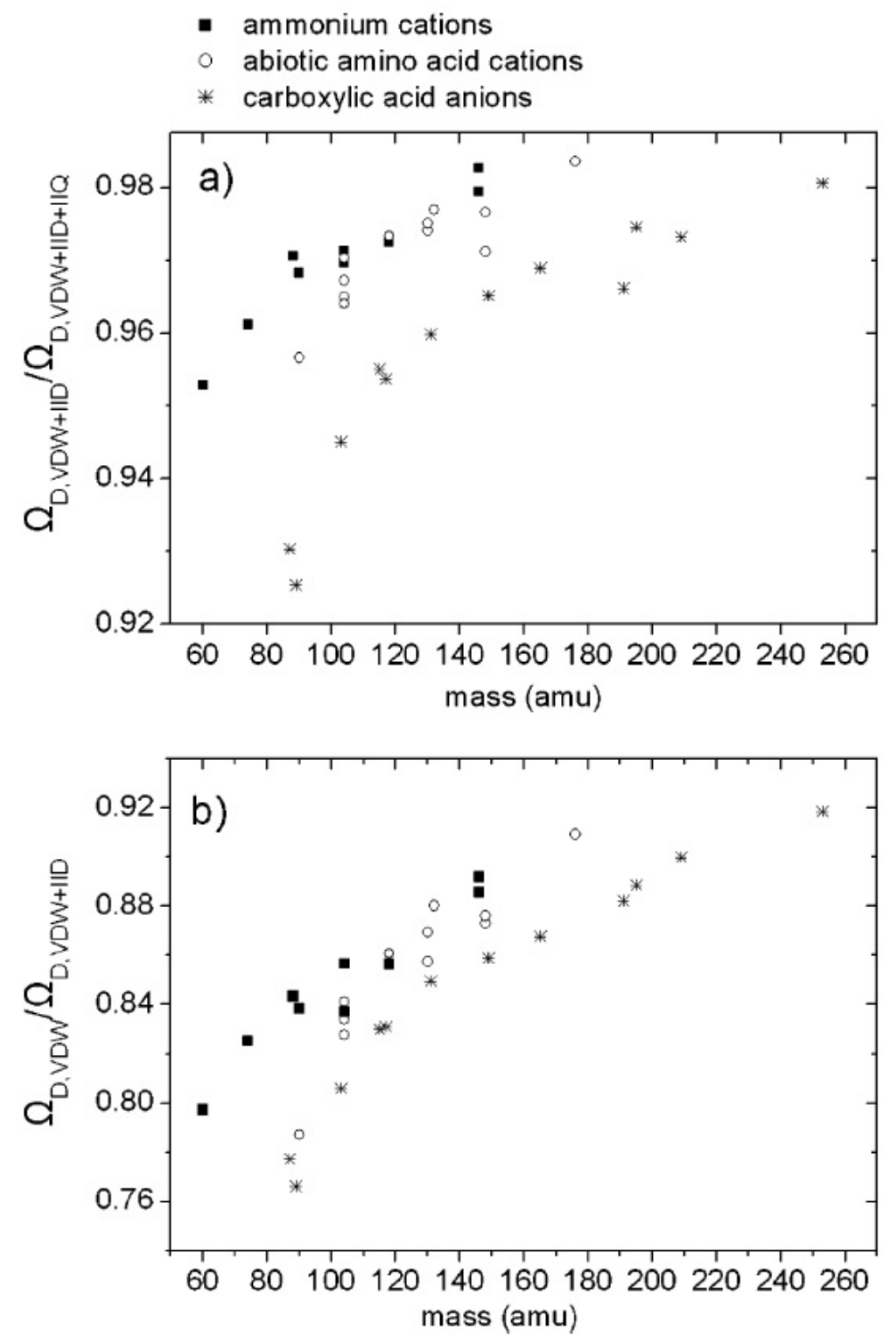

Figure 5.4: Plots of theoretically determined collision cross-sections $\left(\Omega_{D}\right)$ (a) with potential from van der Waals and ion-induced dipole $(V D W+I I D)$ interactions over the theoretical $\Omega_{D}$ with original pairwise potential, van der Waals + ion-induced dipole + ion-quadrupole $(V D W+I I D+I Q)$ interactions, and (b) with potential from van der Waals potential $(V D W)$ over the theoretical $\Omega_{D}$ with potential from van der Waals and ion-induced dipole $(V D W+I I D)$ interactions of $3^{\circ}$ and $4^{\circ}$ ammonium cations, abiotic amino acid cations, and carboxylic acid anions in $\mathrm{N}_{2}$ versus ion mass. The ammonium cation data, the abiotic amino acid cation, and carboxylic acid anion data are shown as solid squares, empty circles, and asterisks, respectively. 
Table 5.2: Critical Impact Parameter, $b^{*}$, Langevin Capture Cross-Section, $\Omega_{L}$, and Mean Relative Kinetic Energies, KE, during the Experiments with Experimentally Determined Hard-Sphere Collision Radius, $R_{c}$ for Each Ammonium Cation

\begin{tabular}{lcccc}
\hline \hline ammonium cation & $\begin{array}{c}\mathrm{KE} \\
(\mathrm{kcal} / \mathrm{mol})\end{array}$ & $\begin{array}{c}b^{*} \\
(\AA)\end{array}$ & $\begin{array}{c}\Omega_{L} \\
\left(\AA^{2}\right)\end{array}$ & $\begin{array}{c}R_{c} \\
(\AA)\end{array}$ \\
\hline trimethylammonium & 1.70 & 5.08 & 81.0 & 5.41 \\
tetramethylammonium & 1.69 & 5.09 & 81.3 & 5.48 \\
trimethylethylammonium & 1.67 & 5.10 & 81.7 & 5.57 \\
$N, N$-dimethylammoniumethanol & 1.67 & 5.10 & 81.7 & 5.55 \\
choline & 1.65 & 5.11 & 82.1 & 5.66 \\
$N, N$-dimethylglycine & 1.65 & 5.11 & 82.1 & 5.67 \\
betaine & 1.64 & 5.12 & 82.5 & 5.75 \\
acetylcholine & 1.60 & 5.15 & 83.4 & 6.01 \\
(3-carboxypropyl)trimethylammonium & 1.60 & 5.15 & 83.5 & 6.01 \\
\hline \hline
\end{tabular}

between the $\Omega_{D, V D W}$ of ions and the $\Omega_{D, V D W+I I D}$ increases from 75 to $92 \%$ along with the mass of the molecular ion increases (Figure 5.4b). This is easy to rationalize since the contribution of the van der Waals interaction increases as the size (i.e., number of atoms) of the molecular ion increases. As a result, it can be concluded that the contribution of long-range ion-induced dipole interaction is important for the $\Omega_{D}$ of small size molecular ions, while the van der Waals interaction prominently affects to the $\Omega_{D}$ in large size molecular ions in this study.

\subsubsection{Van der Waals Potential}

The plots of the $\Omega_{D}$ of $3^{\circ}$ and $4^{\circ}$ ammonium cations, abiotic amino acid cations, and carboxylic acid anions determined only with the van der Waals potential versus ion mass are shown in Figure 5.5, providing the comparison with the corresponding $\Omega_{D}$ from original pairwise potential, which is the combined potential of van der Waals, ion-induced dipole, and ion-quadrupole interactions. It is notable that the characteristic relative $\Omega_{D, V D W}$ show high similarity to the relative $\Omega_{D}$ from the original theoretical calculations. It is inferred that the distinction between the $\Omega_{D}$ for each ion is largely due to the short-range van der Waals interaction between ion and neutral $\mathrm{N}_{2}$ molecule. The molecular weight and specific geometry of the ions is considered to dominate the short-range van der Waals interaction, which affect the collision cross-section of the ion.

\subsubsection{Mass-Mobility Correlation}

It has been suggested from the classical ion-neutral collision calculation that our ion-neutral collision occurs at the borderline between systems dominated by either long-range or short-range interactions. This is well supported from the theoretical investigation using the TJ method. The contribution of long-range interaction to the $\Omega_{D}$ of ammonium cations is large $(\sim 30 \%)$ for small ions and decreases 


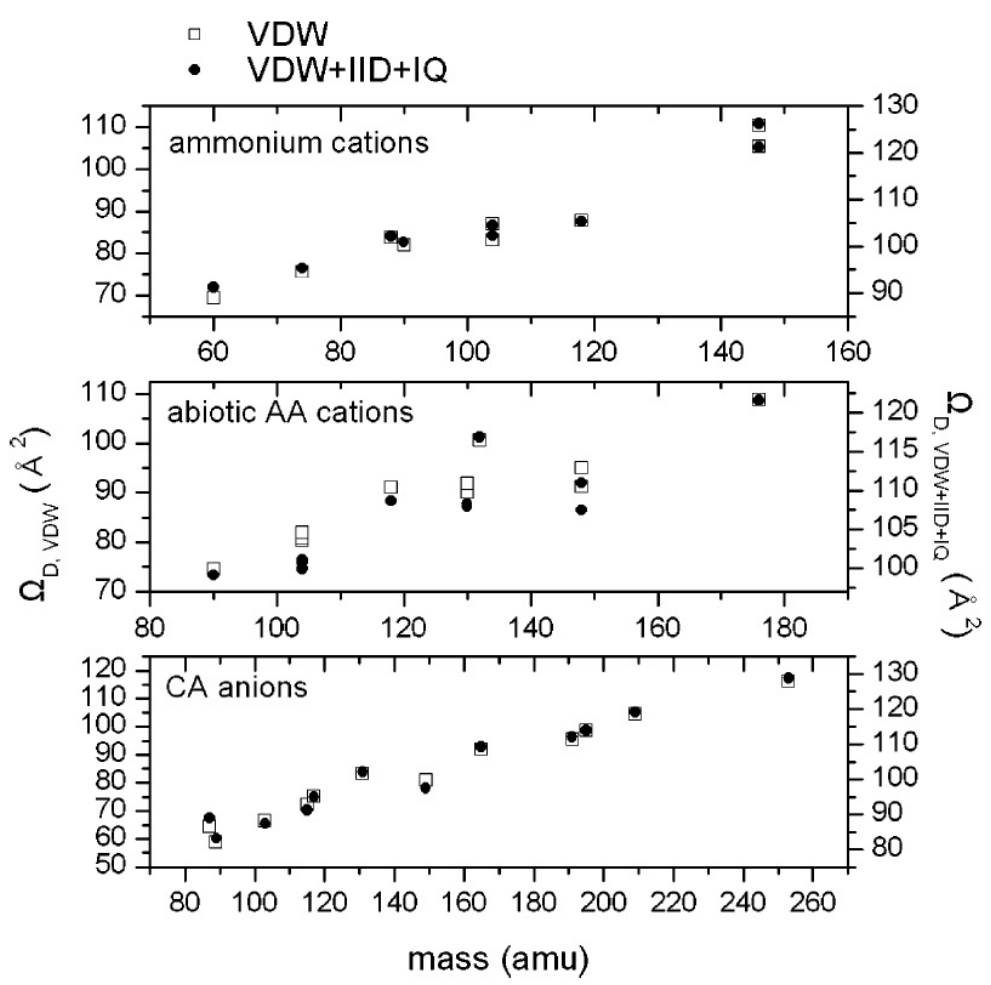

Figure 5.5: Plots of theoretically determined collision cross-sections $\left(\Omega_{D}\right)$ of $3^{\circ}$ and $4^{\circ}$ ammonium cations, abiotic amino acid cations, and carboxylic acid anions in $\mathrm{N}_{2}$ versus ion mass. The calculated $\Omega_{D}$ of the molecular ions only with van der Waals $(V D W)$ interaction with $\mathrm{N}_{2}$ are shown as empty squares (left $y$-axis). The calculated $\Omega_{D}$ of the molecular ions with original pairwise potential, van der Waals + ion-induced dipole + ion-quadrupole $(V D W+I I D+I Q)$ interactions, with $\mathrm{N}_{2}$ are shown as solid circles (right $y$-axis).

Table 5.3: Theoretically Determined Collision Cross-Sections of $3^{\circ}$ and $4^{\circ}$ Ammonium Cations

\begin{tabular}{lcccc}
\hline \hline ammonium cation & $\begin{array}{c}{ }^{a} \Omega_{D} \\
\left({ }^{b} \mathrm{VDW}+\right. \\
\left.{ }^{c} \mathrm{IID}+{ }^{d} \mathrm{IQ}\right)\end{array}$ & $\begin{array}{c}{ }^{a} \Omega_{D} \\
\left({ }^{b} \mathrm{VDW}+\right. \\
\left.{ }^{c} \mathrm{IID}\right)\end{array}$ & $\begin{array}{c}{ }^{a} \Omega_{D} \\
\left({ }^{b} \mathrm{VDW}\right)\end{array}$ & $\begin{array}{c}{ }^{a} \Omega_{D} \\
\left({ }^{e} \text { center }\right. \\
\text { charge })\end{array}$ \\
\hline trimethylammonium & 91.2 & 86.9 & 69.3 & 91.3 \\
tetramethylammonium & 95.3 & 91.6 & 75.6 & 95.1 \\
trimethylethylammonium & 102.2 & 99.2 & 83.7 & 101.0 \\
$N, N$-dimethylammoniumethanol & 100.9 & 97.7 & 81.9 & 100 \\
choline & 104.5 & 101.5 & 87.0 & 104 \\
$N, N$-dimethylglycine & 102.3 & 99.2 & 83.0 & 101.7 \\
betaine & 105.3 & 102.4 & 87.7 & 105.3 \\
acetylcholine & 126.3 & 123.7 & 110.3 & 120.2 \\
(3-carboxypropyl)trimethylammonium & 121.1 & 119.0 & 105.4 & 117.8 \\
\hline \hline
\end{tabular}

${ }^{a}$ Collision cross-section $\left(\AA^{2}\right) . \quad{ }^{b}$ Van der Waals potential. ${ }^{c}$ Ion-induced dipole interaction. ${ }^{d}$ Ionquadrupole interaction. ${ }^{e}$ Ionic charge at center of mass. 
to less than $10 \%$ as the size of the ion increases.

Previous studies have suggested that charge localization on certain functional groups and the specific structure of the ion play major roles in the interaction between ions and neutral gas molecules in IMS $[7,8,9]$. In order to assess the effect of specific charge distribution in the molecular ion on $\Omega_{D}$, the ionic $\Omega_{D}$ were evaluated after assigning the charge of the molecular ion at the center of mass. In general, $\Omega_{D}$ of ions, in which a total charge +1 has been assigned at the center of mass in the molecule exhibit insignificant deviations from the $\Omega_{D}$ of the ions determined with DFT calculated Mulliken charge distributions. The $\Omega_{D}$ of the ammonium cations with the charge at the center of mass show an average deviation of $0.7 \%$ from the $\Omega_{D}$ of ions with Mulliken charge distributions (Table 5.3). The $\Omega_{D}$ of the carboxylic acid anions and abiotic amino acid cations exhibit 0.64 and $2.7 \%$ deviations, respectively, between the two models. This implies that the influence of the ion charge distribution on $\Omega_{D}$ is minimal. The distance of the center of charge from the center of mass was calculated to investigate the specific charge distribution of the molecular ion in the present study. The average distance between the centers of charge from the centers of mass in the molecular ions is $0.7 \AA$ for ammonium cations, and $0.9 \AA$ for abiotic amino acid cations and carboxylic acid anions. It is inferred that the sizes of the molecular ions investigated in this study are too small to expect localization of the charge to a specific site.

In the previous section, we discussed that all potential terms, ion-quadrupole, ion-induced dipole, and van der Waals potential, are important considerations in determining the collision cross-section of the ions. Especially 75-95\% of collision cross-section is contributed by van der Waals interactions, which implies that strong mass-mobility correlations are highly affected by the geometries of the ions. This can explain the correlation observed in previous studies such as carboxylic acids and amino acids in terms of their structural similarity $[14,15]$. However, it is not able to explain the strong correlation among the ammonium cations. Localization of the charge in molecular ions induces specific gas-phase intramolecular cyclic structures of deprotonated carboxylate anions [15, 42] and protonated abiotic amino acid cations [14]. However, DFT optimized structures of highly alkylated ammonium cations show no significant influence of the localization of the charge on the structures (Figure 5.2).

To evaluate the pure geometrical effect on the $\Omega_{D}$, we calculated the molecular volume and surface area of ions in $\mathrm{N}_{2}$, which are also known as solvent-excluded volume and area [43], using the Maximal Speed Molecular Surface (MSMS) program [44]. The volume and surface area of ion are traced by the inward-facing part of the probe sphere as it rolls over the ion [43]. The radius of the probe sphere is set to be the hard-sphere diameter of $\mathrm{N}_{2}$ molecule, $3.70 \AA$. A distinct mass-volume correlation among the ammonium cations with different numbers of oxygen atoms is found. However, the surface area demonstrates a higher correlation with ion mass for the overall mass range. For example, the volume increases 7.6 and $5.6 \%$ from trimethylethylammonium (88 amu) to choline 
89

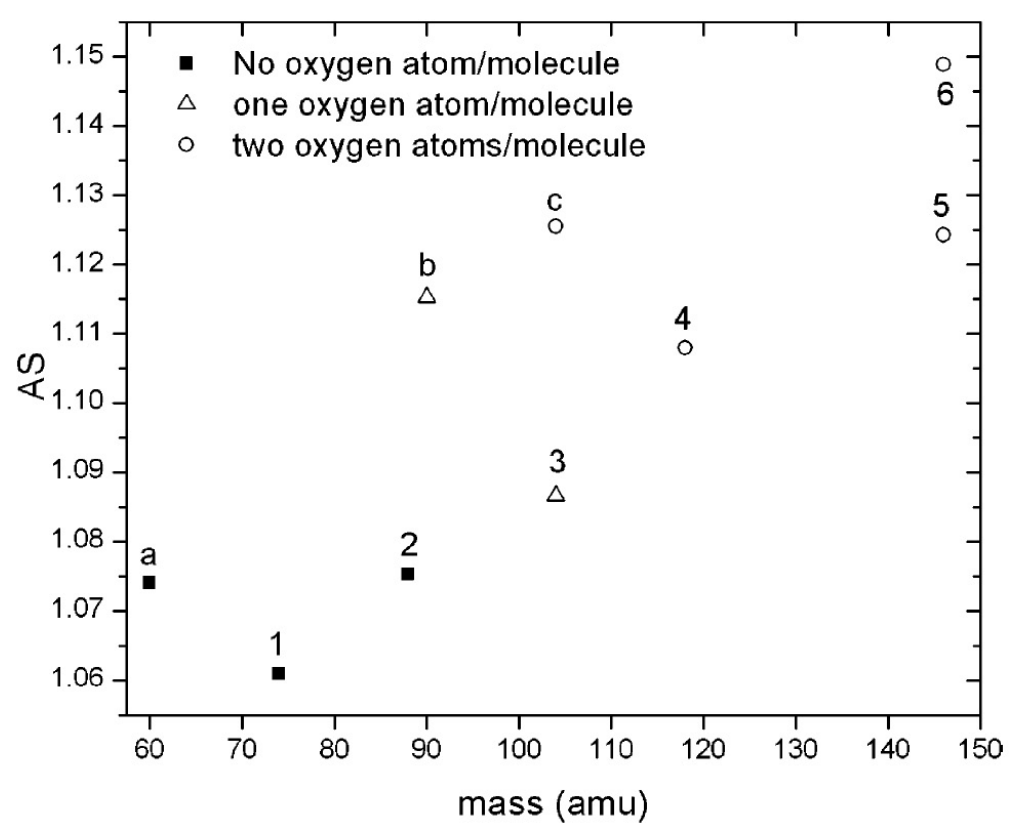

Figure 5.6: Plot of the total shape asymmetry (AS) of the ammonium cations versus ion mass. The ammonium cations with no oxygen atom are shown as solid squares. The ions containing one oxygen atom and two oxygen atoms are shown as empty triangles and empty circles, respectively. The DFT optimized structure of each numerically or alphabetically labeled ion is shown in Figure 5.2.

(104 amu) and betaine (118 amu) while the surface area increases 6.1 and $6.8 \%$, respectively. Using the obtained molecular volume and surface area, the molecular ion's asymmetry of the total shape is determined (Figure 5.6). The asymmetry of the total shape (AS) is expressed as

$$
\mathrm{AS}=\left(\frac{S}{4 \pi}\right)\left(\frac{3 V}{4 \pi}\right)^{-2 / 3}=\frac{1}{4.836}\left(\frac{S}{V^{2 / 3}}\right)
$$

where $S$ and $V$ are molecular surface area and volume, respectively. When the molecular ion is symmetrical (i.e., spherical) AS becomes unity, with AS increasing from unity as the asymmetry in shape increases. As seen in Figure 5.6, higher asymmetry is observed as the number of oxygen atoms and the size of the ion increase. Although the larger content of oxygen atom makes for smaller molecular volumes, it increases the asymmetry of the total shape, which increases the surface area of the ion. It is therefore inferred that our observed strong mass-mobility correlation is largely due to geometrical factors. This allows us to comprehend the observed mass-mobility correlation among two different classes of ammonium cations with the heteroatom complements in the present study.

\subsection{Conclusions}

A high correlation between mass and mobility in $\mathrm{N}_{2}$ is observed from a number of tertiary and quaternary ammonium cations. The classical ion-neutral collision calculation implies that the group 
of molecules studied here are on the borderline between being dominated by long-range versus shortrange interactions, favoring some orbiting at lower collision energies, which would then determine the cross-section. Theoretical investigation using a modified trajectory (TJ) method also indicates that all potential terms, ion-quadrupole, ion-induced dipole, and van der Waals potential, are important considerations in determining the collision cross-section of the ions. For the smaller molecular ions, the importance of long-range interaction is emphasized, while short-range interactions dominate the collision cross-sections of the larger molecular ions. The evaluated volume and surface area suggest that shape asymmetry of the ammonium cations plays a small but significant role in determining the observed correlation between mass and mobility. The increase of the asymmetry in the shape of an ion compensates the reduction of the ion's volume, which finally yields similar mobilities of the ammonium cations with similar molecular weight investigated in this study, independent of their heteroatom complement.

\subsection{Acknowledgments}

This research was carried out at the Jet Propulsion Laboratory, California Institute of Technology, under a contract with the National Aeronautics and Space Administration (NASA), the Noyes Laboratory of Chemical Physics, California Institute of Technology, and the Material and Process Simulation Center, Beckman Institute, California Institute of Technology. Financial support through NASA's Astrobiology Science and Technology Instrument Development, Planetary Instrument Definition and Development, and Mars Instrument Development programs is gratefully acknowledged. We appreciate the support provided by the Mass Spectrometry Resource Center in the Beckman Institute. The authors greatly appreciate Prof. Martin Jarrold at Indiana University Bloomington for generously allowing us to use and modify the Mobcal program. Hyungjun Kim and Hugh I. Kim contributed equally to this work. 


\section{Bibliography}

[1] Fenn, J. B.; Mann, M.; Meng, C. K.; Wong, S. F.; Whitehouse, C. M. Science 1989, 246, 64-71.

[2] Shumate, C. B.; Hill, H. H. Anal. Chem. 1989, 61, 601-606.

[3] Wittmer, D.; Luckenbill, B. K.; Hill, H. H.; Chen, Y. H. Anal. Chem. 1994, 66, 2348-2355.

[4] Creaser, C. S.; Griffiths, J. R.; Bramwell, C. J.; Noreen, S.; Hill, C. A.; Thomas, C. L. P. Analyst 2004, 129, 984-994.

[5] Gidden, J.; Ferzoco, A.; Baker, E. S.; Bowers, M. T. J. Am. Chem. Soc. 2004, 126, 15132-15140.

[6] Julian, R. R.; Hodyss, R.; Kinnear, B.; Jarrold, M. F.; Beauchamp, J. L. J. Phys. Chem. B 2002, 106, 1219-1228.

[7] Counterman, A. E.; Clemmer, D. E. J. Phys. Chem. B 2001, 105, 8092-8096.

[8] Kaleta, D. T.; Jarrold, M. F. J. Phys. Chem. A 2002, 106, 9655-9664.

[9] Wu, C.; Siems, W. F.; Klasmeier, J.; Hill, H. H. Anal. Chem. 2000, 72, 391-395.

[10] Shelimov, K. B.; Clemmer, D. E.; Hudgins, R. R.; Jarrold, M. F. J. Am. Chem. Soc. 1997, $119,2240-2248$.

[11] Hudgins, R. R.; Woenckhaus, J.; Jarrold, M. F. Int. J. Mass Spectrom. 1997, 165, 497-507.

[12] Clemmer, D. E.; Jarrold, M. F. J. Mass Spectrom. 1997, 32, 577-592.

[13] Beegle, L. W.; Kanik, I.; Matz, L.; Hill, H. H. Anal. Chem. 2001, 73, 3028-3034.

[14] Johnson, P. V.; Kim, H. I.; Beegle, L. W.; Kanik, I. J. Phys. Chem. A 2004, 108, 5785-5792.

[15] Kim, H. I.; Johnson, P. V.; Beegle, L. W.; Beauchamp, J. L.; Kanik, I. J. Phys. Chem. A 2005, 109, 7888-7895.

[16] Gidden, J.; Bowers, M. T. Eur. Phys. J. D. 2002, 20, 409-419.

[17] Asbury, G. R.; Klasmeier, J.; Hill, H. H. Talanta 2000, 50, 1291-1298. 
[18] Asbury, G. R.; Wu, C.; Siems, W. F.; Hill, H. H. Anal. Chim. Acta 2000, 404, 273-283.

[19] Griffin, G. W.; Dzidic, I.; Carroll, D. I.; Stillwel, R. N.; Horning, E. C. Anal. Chem. 1973, 45, 1204-1209.

[20] Berant, Z.; Karpas, Z. J. Am. Chem. Soc. 1989, 111, 3819-3824.

[21] Karpas, Z.; Berant, Z. J. Phys. Chem. 1989, 93, 3021-3025.

[22] Steiner, W. E.; English, W. A.; Hill, H. H. J. Phys. Chem. A 2006, 110, 1836-1844.

[23] Vonhelden, G.; Hsu, M. T.; Kemper, P. R.; Bowers, M. T. J. Chem. Phys. 1991, 95, 3835-3837.

[24] Mesleh, M. F.; Hunter, J. M.; Shvartsburg, A. A.; Schatz, G. C.; Jarrold, M. F. J. Phys. Chem. 1996, 100, 16082-16086.

[25] Mason, E. A.; O’hara, H.; Smith, F. J. J. Phys. B 1972, 5, 169-176.

[26] Blusztajn, J. K. Science 1998, 281, 794-795.

[27] Zeisel, S. H.; Dacosta, K. A.; Youssef, M.; Hensey, S. J. Nutr. 1989, 119, 800-804.

[28] McHowat, J.; Jones, J. H.; Creer, M. H. J. Lipid Res. 1996, 37, 2450-2460.

[29] Wu, C.; Siems, W. F.; Asbury, G. R.; Hill, H. H. Anal. Chem. 1998, 70, 4929-4938.

[30] Asbury, G. R.; Hill, H. H. Anal. Chem. 2000, 72, 580-584.

[31] Becke, A. D. J. Chem. Phys. 1993, 98, 5648-5652.

[32] Lee, C. T.; Yang, W. T.; Parr, R. G. Phys. Rev. B 1988, 37, 785-789.

[33] Harihara, P. C.; Pople, J. A. Chem. Phys. Lett. 1972, 16, 217-219.

[34] Olney, T. N.; Cann, N. M.; Cooper, G.; Brion, C. E. Chem. Phys. 1997, 223, 59-98.

[35] Rappe, A. K.; Casewit, C. J.; Colwell, K. S.; Goddard, W. A.; Skiff, W. M. J. Am. Chem. Soc. 1992, 114, 10024-10035.

[36] Graham, C.; Imrie, D. A.; Raab, R. E. Mol. Phys. 1998, 93, 49-56.

[37] Dugan, J. V.; Palmer, R. W. Chem. Phys. Lett. 1972, 13, 144-149.

[38] Dugan, J. V.; Magee, J. L. J. Chem. Phys. 1967, 47, 3103-3112.

[39] Bowers, M. T. Gas Phase Ion Chemistry; Academic Press: New York, 1979; Vol. 1.

[40] Wannier, G. H. Bell Syst. Tech. J. 1953, 32, 170-254. 
[41] Su, T.; Bowers, M. T. Int. J. Mass Spectrom. Ion Processes 1975, 17, 309-319.

[42] Woo, H. K.; Wang, X. B.; Lau, K. C.; Wang, L. S. J. Phys. Chem. A 2006, 110, 7801-7805.

[43] Connolly, M. L. J. Am. Chem. Soc. 1985, 107, 1118-1124.

[44] Sanner, M. F.; Olson, A. J.; Spehner, J. C. Biopolymers 1996, 38, 305-320. 


\section{Chapter 6}

\section{Structural Characterization of Unsaturated Phospholipids Using Traveling Wave Ion Mobility Spectrometry}

\subsection{Abstract}

A number of phosphatidylcholine (PC) cations spanning a mass range of 400 to 1000 Da are investigated using electrospray ionization mass spectrometry coupled with traveling wave ion mobility spectrometry (TWIMS). A high correlation between mass and mobility is demonstrated with saturated phosphatidylcholine cations in $\mathrm{N}_{2}$. A significant deviation from this mass-mobility correlation line is observed for the unsaturated PC cation. We found that the double bond in the acyl chain causes a $5 \%$ reduction in drift time. The drift time is reduced at a rate of $\sim 1 \%$ for each additional double bond. Theoretical collision cross-sections of PC cations exhibit good agreement with experimentally evaluated values. Collision cross-sections are determined using the recently derived relationship between mobility and drift time in TWIMS stacked ring ion guide (SRIG) and compared to estimate collision cross-sections using empiric calibration method. Computational analysis was performed using the modified trajectory (TJ) method with nonspherical $\mathrm{N}_{2}$ molecules as the drift gas. The difference between estimated collision cross-sections and theoretical collision cross-sections of PC cations is related to the sensitivity of the PC cation collision cross-sections to the details of the ion-neutral interactions. The origin of the observed correlation and deviation between mass and mobility of PC cations is discussed in terms of the structural rigidity of these molecules using molecular dynamics simulations. 


\subsection{Introduction}

Lipids are essential biological components and have critical roles for cell structure, energy storage, and metabolic control [1]. Characterizing their structures is an essential part of lipid analysis. In addition, searching for lipid molecules is a valuable strategy for finding traces of extinct or extant life elsewhere in outer space. Lipids and biomembranes can be preserved for a long period; thus, detailed characterization of these biomarker compositions allows for the assessment of major contributing species [2]. Lipids offer records of modern and ancient life, environmental conditions, and changes in history. However, the variety and in situ alteration of lipids also increases complexity, making them difficult to characterize fully [3].

The separation and characterization of phospholipids using tandem ion mobility mass spectrometry (IM-MS) has been investigated by several research groups [4, 5, 6, 7]. Utilizing matrix-assisted laser desorption ionization (MALDI) with IM-MS, phospholipid ions have been separated from other biomolecule ions $[4,8]$. Separation can be achieved based on the correlation between mass and ion mobility. Phospholipids in tissue samples have been directly analyzed using MALDI-IM-MS [4, 5]. These studies have reported that phospholipid ions have slower mobility than peptide, carbohydrate, and nucleotide ions with similar masses $[4,7,8,9]$. In general, peptides, nucleotides, and carbohydrates form globular conformations in the gas phase due to intramolecular Coulombic interactions $[10,11,12]$. However, such interactions are difficult to achieve for phospholipid molecules because their major components are aliphatic acyl chains. Recently, Jackson et al. reported the effects of various head and tail groups of phospholipids on mass-mobility correlations using MALDI-IM-MS [6]. They report a slight increase in the mobility of phospholipids as the degree of unsaturation on the acyl chain increases.

The correlation between the mass and mobility of molecular ions has been used to separate and characterize ions related to the mobility of gas phase ion molecules. In the early 1970s, Griffin et al. [13] showed that mass and mobility are strongly correlated for structurally related compounds. In the late 1980s, Karpas and Berant demonstrated distinct mass-mobility correlations of acetyls, aromatic amines, and aliphatic amines drifting in various drift gases including $\mathrm{He}, \mathrm{N}_{2}, \mathrm{CO}_{2}$, and air $[14,15]$. Clemmer and co-workers have demonstrated distinct mass-mobility correlations for peptides with molecular weights of 500 to 2500 Da [16]. Recently, Thalassinos et al. characterized phosphorylated peptides as having higher mobilities than their non-phosphorylated counterparts [17].

Our laboratory has investigated the distinct mass-mobility correlations of amino acids and carboxylic acids drifting in $\mathrm{N}_{2}$ and $\mathrm{CO}_{2}[18,19]$. Recently, we experimentally observed a high correlation between mass and mobility of tertiary and quaternary ammonium cations in $\mathrm{N}_{2}$ [20]. This observed correlation was investigated using classical ion-neutral collision dynamic theories and computational 
calculation using a modified trajectory (TJ) method. From these theoretical investigations, the ammonium cations in the mass range from $60 \mathrm{Da}$ to $150 \mathrm{Da}$ are on the borderline between being dominated by long range versus short range interactions with $\mathrm{N}_{2}$. In addition, all potential terms, ion-quadrupole, ion-induced dipole, and van der Waals potential are important considerations for determining the collision cross-sections of the ions in $\mathrm{N}_{2}$.

In this paper, we measure drift times for a number of phosphatidylcholines (PC) spanning a mass range of 400 to $1000 \mathrm{Da}$ in $\mathrm{N}_{2}$ using a commercial traveling wave ion mobility spectrometry (TWIMS) coupled with orthogonal acceleration time-of-flight (oa-TOF) mass spectrometry (Waters Synapt HDMS). Of particular interest is the possible dependence of mass-mobility correlations on the symmetry, length, and degree of saturation of the acyl chains. Despite a wide range of TWIMS applications in various chemistry fields $[17,21,22,23,24,25]$, studies have only begun to understand the principal physics behind the TWIMS drift time and ion mobility. A number of studies have employed the empiric calibration method to estimate mobilities and collision cross-sections of analyte ions from the drift times in TWIMS [17, 21, 22, 25, 26]. Recently, Shvartsburg and Smith quantitatively revealed the relationship between drift time and ion mobility in TWIMS [27].

Jarrold and co-workers have proposed a TJ method based on a soft-core ion-neutral interaction potential to interpret collision cross-sections of ion molecules [28]. A modified TJ method for the ion-neutral interaction to account for the potential associated with the non-spherical drift gas $\mathrm{N}_{2}$ has been applied to predict collision cross-sections of PC cations and to test the sensitivity of these cross-sections in order to detail the structural rigidity of these molecules [20]. Results from the estimated collision-cross sections using empiric calibration are compared with the evaluated relationship between TWIMS drift time and mobility by Shvartsburg and Smith [27]. The origin of the observed correlation and deviation between PC mass and mobility is discussed.

\subsection{Experimental Section}

\subsubsection{Chemicals and Reagents}

All phosphatidylcholines studied in this work were purchased from Avanti Polar Lipids (Alabaster, $\mathrm{AL}$ ) and were used without further purification. All solvents (water, methanol, and formic acid) were HPLC grade and were purchased from EMD Chemicals Inc. (Gibbstown, NJ). Calibrant peptides (GGGGGG and AAAAAA), cytochrome C, and trypsin from porcine pancreas were purchased from Sigma-Aldrich (St. Louis, MO). Samples were prepared by dissolving known quantities of molecules in a solvent consisting of $1: 1$ water and methanol with $0.1 \%$ formic acid by volume to yield sample concentrations in the range of $50 \mu \mathrm{M}$. Trypsin digest of cytochrome $\mathrm{C}$ was prepared by incubating $200 \mu \mathrm{M}$ of cytochrome $\mathrm{C}$ with $6 \mu \mathrm{g}$ of trypsin from porcine pancreas in $1 \mathrm{~mL}$ of water containing $25 \mathrm{mM}$ ammonium bicarbonate $\left(\mathrm{NH}_{4} \mathrm{HCO}_{3}\right)$ at $37^{\circ} \mathrm{C}$ for 4 hours. The trypsin was then removed 
using a Millipore Microcon centrifugal filter fitted with an Ultracel YM-10 membrane. The sample solution was diluted to an appropriate concentration for ESI with 1:1 water/methanol and $0.1 \%$ formic acid by volume. Phospholipid and peptide ions examined in this study are listed in Table 6.1 along with their respective molecular weights.

Phosphatidylcholines examined in this study are named by their acyl chain length and number of double bonds. For example, 1-steroyl-2-oleoyl-sn-phosphatidylcholine (SOPC), which comprises two 18 carbon acyl chains and one double bond, is referred to as 18:0-18:1 PC.

\subsubsection{Electrospray Ionization Traveling Wave Ion Mobility Mass Spec- trometer}

Experiments were performed on a Synapt HDMS traveling wave ion mobility orthogonal acceleration time-of-flight (TW-IM-oa-TOF, Waters, Manchester, U.K.) in positive ion mode. The details of the instrument have been described elsewhere [29, 30]. Source temperature of $100^{\circ} \mathrm{C}$, capillary voltage of $3 \mathrm{kV}$, desolvation temperature of $250^{\circ} \mathrm{C}$, and cone voltage of $30 \mathrm{~V}$ were set as parameters for ESI. Other parameters of the instrument were optimized to achieve the best separation of phospholipids without the roll-over effect [29]. Nitrogen drift gas was introduced to the TWIMS stacked ring ion guide (SRIG) at a $25 \mathrm{~mL} / \mathrm{min}$ flow rate, which corresponds to 0.39 Torr. The traveling wave (T-wave) height and velocity were optimized as $8 \mathrm{~V}$ and $300 \mathrm{~m} / \mathrm{s}$, respectively. For each sample, 150 spectra were obtained and averaged for analysis. The drift times of the singly charged phospholipid cations and peptides were determined from the location of the ion mobility peak maxima extracted manually using MassLynx (v 4.1) software (Waters corp. Milford, MA). Resolution of the instrument was found to be $\sim 0.8 \mathrm{~ms}$ in full width at half maximum (FWHM) with drift times for the ions studied and the parameters employed in this study.

\subsubsection{Collision Cross-Section Calibration}

Previously published collision cross-sections of singly charged peptide hexaglycine, hexaalanine, and tryptic digest of cytochrome $\mathrm{C}$ in helium drift gas were used to create a calibration curve [16]. Recently published PC collision cross-sections determined in helium drift gas were also used for the calibration [9]. The calibration procedure was adopted from Thalassinos et al. [17]. The effective drift time $\left(t_{d}^{\prime \prime}\right)$ of the calibrant was corrected for mass independent and mass dependent time. The published collision cross-section of the calibrant was scaled by reduced mass in $\mathrm{N}_{2}$. The effected drift time was plotted against the corrected published collision cross-section $\left(\Omega_{D}^{\prime}\right)$. The plot was used to fit a linear and power trend. The equation from the fitting result was used to estimate collision cross-sections of phospholipids with reduced mass. 
Table 6.1: Mass, Drift time, and Corrected Drift Time of Ion Molecule Used in This Study

\begin{tabular}{ccccc}
\hline \hline Name & cationization & ${ }^{c}$ mass & $t_{d}(\mathrm{~ms})$ & ${ }^{d} t_{d}^{\prime}(\mathrm{ms})$ \\
\hline${ }^{a}$ 5:0-5:0 PC & $\mathrm{H}^{+}$ & 426 & 3.58 & 3.39 \\
${ }^{a}$ 8:0-8:0 PC & $\mathrm{Na}^{+}$ & 448 & 3.97 & 3.77 \\
& $\mathrm{H}^{+}$ & 510 & 4.99 & 4.78 \\
${ }^{a}$ 11:0-11:0 PC & $\mathrm{Na}^{+}$ & 532 & 5.50 & 5.29 \\
& $\mathrm{H}^{+}$ & 595 & 6.40 & 6.18 \\
${ }^{a}$ 14:0-16:0 PC & $\mathrm{Na}^{+}$ & 617 & 6.85 & 6.62 \\
& $\mathrm{H}^{+}$ & 707 & 8.06 & 7.82 \\
${ }^{a}$ 16:0-16:0 PC & $\mathrm{Na}^{+}$ & 729 & 8.32 & 8.07 \\
& $\mathrm{H}^{+}$ & 735 & 8.64 & 8.39 \\
${ }^{a}$ 18:0-14:0 PC & $\mathrm{Na}^{+}$ & 757 & 8.77 & 8.51 \\
& $\mathrm{H}^{+}$ & 735 & 8.58 & 8.33 \\
${ }^{a}, b$ 16:0-18:2 PC & $\mathrm{Na}^{+}$ & 757 & 8.77 & 8.51 \\
${ }^{a}$ 18:0-16:0 PC & $\mathrm{H}^{+}$ & 759 & 8.51 & 8.26 \\
${ }^{a}$ 16:0-20:4 PC & $\mathrm{H}^{+}$ & 763 & 8.96 & 8.71 \\
${ }^{a}, b$ 18:0-18:2 PC & $\mathrm{Na}^{+}$ & 785 & 9.15 & 8.89 \\
${ }^{a}$ 18:0-18:1 PC & $\mathrm{H}^{+}$ & 783 & 8.58 & 8.32 \\
${ }^{a}$ 18:0-18:0 PC & $\mathrm{H}^{+}$ & 787 & 8.90 & 8.64 \\
${ }^{a}$ 16:0-22:6 PC & $\mathrm{H}^{+}$ & 789 & 8.96 & 8.70 \\
${ }^{a}$ 24:0-24:0 PC & $\mathrm{H}^{+}$ & 791 & 9.41 & 9.15 \\
${ }^{b}$ KK (cytC) & $\mathrm{H}^{+}$ & 807 & 8.83 & 8.57 \\
${ }^{b}$ HK (cytC) & $\mathrm{H}^{+}$ & 959 & 12.2 & 11.9 \\
${ }^{b}$ GKK (cytC) & $\mathrm{H}^{+}$ & 275 & 1.86 & 1.70 \\
${ }^{b}$ GGGGGG & $\mathrm{H}^{+}$ & 284 & 1.86 & 1.70 \\
${ }^{b}$ ATNE (cytC) & $\mathrm{H}^{+}$ & 332 & 2.37 & 2.20 \\
${ }^{b}$ AAAAAA & $\mathrm{H}^{+}$ & 362 & 2.18 & 2.00 \\
${ }^{b}$ KATNE (cytC) & $\mathrm{H}^{+}$ & 434 & 3.20 & 3.01 \\
${ }^{b}$ Ac-GDVEK (cytC) & $\mathrm{H}^{+}$ & 446 & 3.14 & 2.94 \\
${ }^{b}$ GITWK (cytC) & $\mathrm{H}^{+}$ & 563 & 4.29 & 4.07 \\
${ }^{b}$ IFVQK (cytC) & $\mathrm{H}^{+}$ & 590 & 4.80 & 4.58 \\
${ }^{b}$ YIPGTK (cytC) & $\mathrm{H}^{+}$ & 605 & 5.12 & 4.89 \\
${ }^{+}$MIFAGIK (cytC) & $\mathrm{H}^{+}$ & 635 & 5.70 & 5.46 \\
\hline
\end{tabular}

${ }^{a}$ Molecules examined in this study. ${ }^{b}$ Molecules used as calibrant. ${ }^{c}$ All ions are singly charged. ${ }^{d}$ Mass dependent corrected drift time. 


\subsubsection{Computational Modeling}

Collision cross-sections of ions were calculated using the modified TJ method [20], which consists of two potential terms representing van der Waals and ion-induced dipole interactions characterized by Lennard-Jones parameters and neutral polarizability, respectively [28]. The modified TJ method describes the interaction between ions and an $\mathrm{N}_{2}$ drift gas that expands applicability beyond cases of ions drifting in He (details of this modification can be found elsewhere) [20]. In brief, we set the polarizability of neutral gas for $\mathrm{N}_{2}\left(1.710 \times 10^{-24} \mathrm{~cm}^{2}\right)$. Due to the linear geometry of $\mathrm{N}_{2}$, two more consequences were taken into account: ion-quadrupole interaction and molecule orientation. The ion-quadrupole interaction is expressed in simple summations of partial charges of negative $q$ $(0.4825 e)$ to each nitrogen atom and one positive $2 q$ at the center of the nitrogen molecule. The orientations of $\mathrm{N}_{2}$ are sampled along the $x$-, $y$-, and $z$-axis; the averaged interaction potential is evaluated using Boltzmann weighting.

In order to consider the effect of structural fluctuation on the collision cross-section at room temperature, we performed NVT molecular dynamics (MD) simulations using a Nosé-Hoover thermostat at $300 \mathrm{~K}$. The inter-atom interactions are described with the all-atom CHARMM PARAM27 force field [31] using the LAMMPS (large-scale atomic/molecular massively parallel simulator) code [32]. We adopted the " $\mathrm{sp}_{2} \mathrm{C}-\mathrm{sp}_{3} \mathrm{C}-\mathrm{sp}_{2} \mathrm{C}$ " angle parameter and the " $\mathrm{sp}_{2} \mathrm{C}-\mathrm{sp}_{2} \mathrm{C}-\mathrm{sp}_{3} \mathrm{C}-\mathrm{sp}_{2} \mathrm{C}$ " dihedral parameters from reference [33], which were optimized using 1, 4-pentadiene. The partial charge distribution of protonated phosphate $\left(\mathrm{O}_{3} \mathrm{P}-\mathrm{O}-\mathrm{H}\right)$ was optimized using Mulliken charge distributions from density functional theory (DFT) calculations (Table 6.2), since the common CHARMM force field only has a partial charge distribution of negatively charged phophate $\left(\mathrm{O}_{3} \mathrm{P}_{-} \mathrm{O}^{-}\right)$. The systems are pre-equilibrated for 100 ps, and the conformations are sampled every one ps from the 200 ps simulations. We note that such a procedure allows for canonical sampling of the conformations at $300 \mathrm{~K}$. We analyzed the collision cross-sections and potential energies of all sampled conformations of PC.

\subsection{Results}

\subsubsection{Saturated Phosphatidylcholine Cations}

The drift times, $t_{d}$, of the PC cations were determined as described above. The drift times were then corrected with the mass dependent flight time, defined as the time that an ion spent in the TOF [17, 26]. Measured and corrected drift times for the PC cations chosen for this study are found in Table 6.1. The corrected drift time from TWIMS was plotted against the mass to charge $(\mathrm{m} / \mathrm{z})$ of the ion, and the plot was used to fit a linear trend. The plot of drift time versus mass for singly protonated PC cations is shown in Figure 6.1 along with the linear fit to the data. As seen 
Table 6.2: Optimized Partial Charge Distribution of Protonated Phosphate from the DFT Calculation of Protonated Dimethyl Phosphate (The Partial Charges with Atom Types for CHARMM Force Field are Tabulated.)

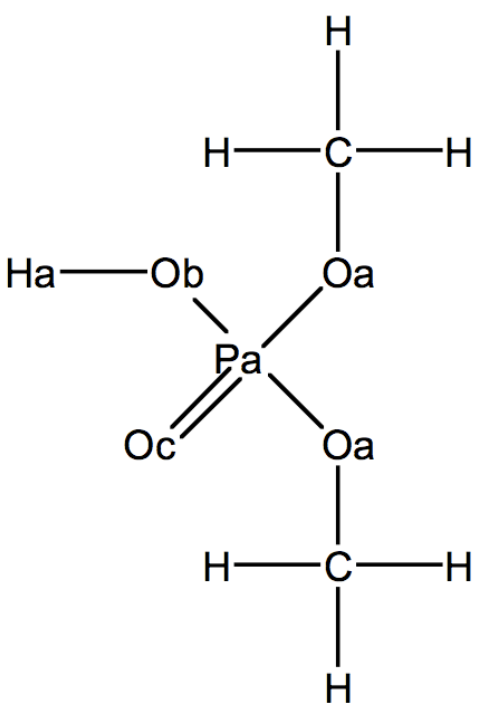

\begin{tabular}{cccc}
\hline \hline & $\begin{array}{c}{ }^{a} \text { Mulliken } \\
\text { Charge }\end{array}$ & $\begin{array}{c}\text { Force Field } \\
\text { Charge }\end{array}$ & $\begin{array}{c}\text { Force Field } \\
\text { Type }\end{array}$ \\
\hline $\mathrm{Pa}$ & 1.16 & 1.50 & $\mathrm{PL}$ \\
$\mathrm{Oa}$ & -0.51 & -0.49 & OSL \\
$\mathrm{Ob}$ & -0.54 & -0.52 & OHL \\
$\mathrm{Oc}$ & -0.57 & -0.56 & O2L \\
$\mathrm{Ha}$ & 0.35 & 0.36 & HOL \\
\hline \hline
\end{tabular}

${ }^{a}$ Computed from density functional theory (DFT) calculation using Jaguar 6.0 of Schrödinger company with B3LYP functional and $6-31 \mathrm{G}^{* *}$ basis set. 
in Figure 6.1a, all saturated PC cations investigated in this study (400 - $1000 \mathrm{Da})$ exhibit a good correlation $\left(R^{2}>0.999\right)$ between mass and drift time (i.e., ion mobility). In particular, symmetry of the two acyl chains in the phospholipid does not affect the common mass-mobility correlation of a saturated PC cation.

\subsubsection{Unsaturated Phosphatidylcholine Cations}

The usual acyl chain length of membrane phospholipids vary from 18 to 20 carbon atoms [34]. Most unsaturated phospholipids contain one acyl chain with one or more cis-double bonds and a saturated one as a second acyl chain [34]. We have selected unsaturated PC cations with these characteristics to investigate the dependence of mass-mobility correlations on the presence of double bonds in the acyl chains of membrane phospholipids (Table 6.1).

Figure 6.1b shows a plot of corrected drift time versus mass for PC cations from $700 \mathrm{Da}$ to 810 Da along with the linear fit to the data. A good correlation $\left(R^{2}=0.984\right)$ is still observed for the saturated PC cations within the mass range. However, a poor correlation between mass and mobility from unsaturated and saturated PC cations is also observed $\left(R^{2}=0.487\right)$. Unsaturated PC cations show higher mobilities (i.e., faster drift time) compared to saturated PC cations. Corrected drift times of 16:0-18:2 PC (MW 759) and 16:0-20:4 PC (MW 783) are measured as $8.26 \mathrm{~ms}$ and $8.32 \mathrm{~ms}$, respectively. They traveled in the SRIG faster than smaller saturated PC cations such as 16:0-16:0 and 18:0-14:0 (MW 735), which have $8.39 \mathrm{~ms}$ and $8.33 \mathrm{~ms}$ drift times, respectively. The corrected drift time of 16:0-22:6 PC (MW 807) is measured as $8.57 \mathrm{~ms}$. Compared to the $8.71 \mathrm{~ms}$ and 9.15 ms, which are corrected drift times of two smaller saturated PC cations, 18:0-16:0 PC (MW 763) and 18:0-18:0 PC (MW 791), respectively, 16:0-22:6 PC travels across the SRIG faster.

The presence of a cis-double bond causes the acyl chain to bend. In addition, a double bond causes a relatively rigid acyl chain structure compared to that of the saturated acyl chain. It is inferred that these two factors cause smaller collision cross-sections and thus faster mobility than unsaturated PC cations.

\subsubsection{Sodiated Phosphatidylcholine Cations}

Figure 6.1c shows the plot of drift time versus mass for protonated and sodiated PC cations. The sodiated PC cations investigated in this study exhibit a good mass-mobility correlation $\left(R^{2}=0.996\right)$ with protonated PC ions. A recent investigation by Kim et al. reported that short range interactions are most important for the collision cross-sections of molecular ions larger than $150 \mathrm{Da}$ [20]. The PC cations (400 - $1000 \mathrm{Da})$ investigated in this study are larger than ions that Kim et al. [20] investigated $(60-250 \mathrm{Da})$. Thus, the importance of short range interactions is emphasized for collision cross-sections of PC cations. In numerous cases, metal cations have been shown to cause 
a

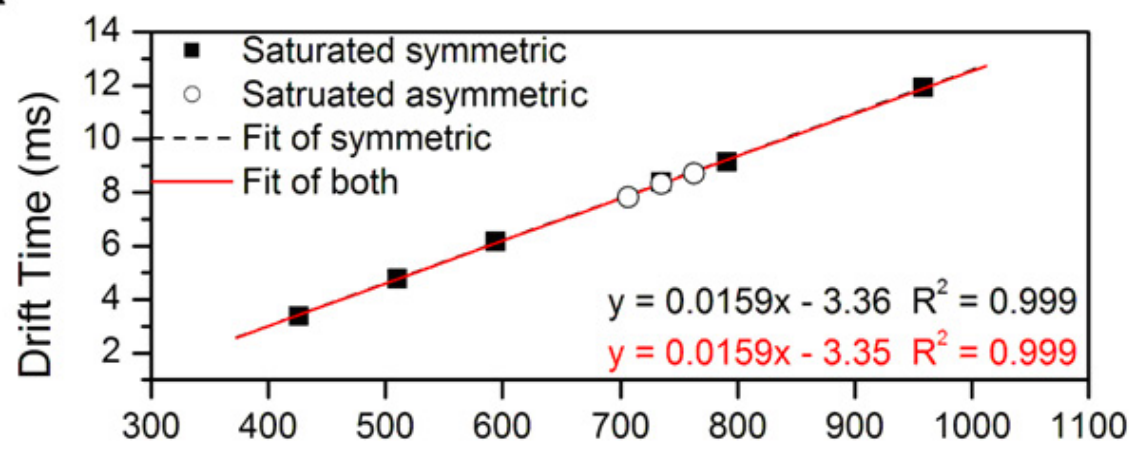

b

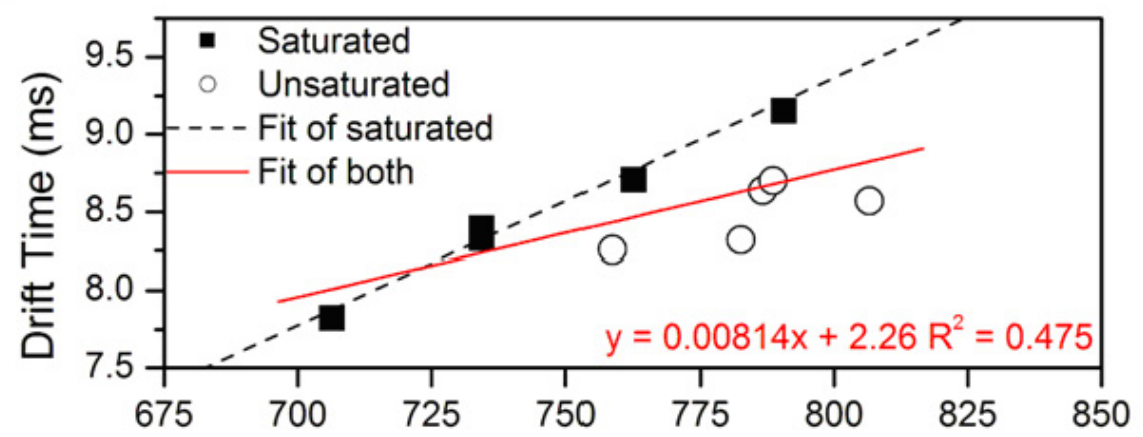

C

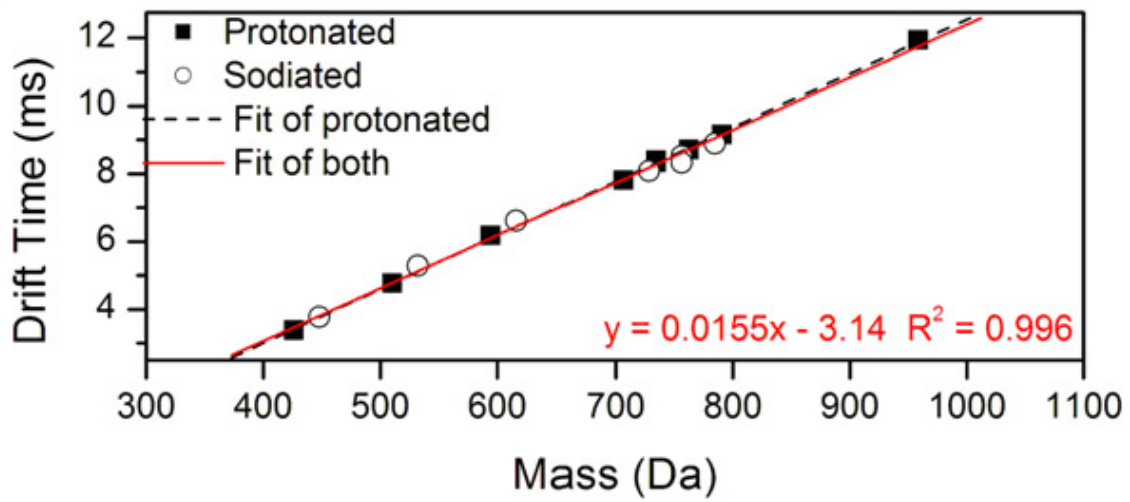

Figure 6.1: (a) Plot of drift time of saturated phosphatidylcholine (PC) cations in traveling wave ion mobility spectrometer versus ion mass. Experimentally determined data for symmetric PC and asymmetric PC cations are shown as solid squares and empty circles, respectively. The black dash and red solid lines are the linear fit to the symmetric PC cation data set and to both symmetric and asymmetric PC cation data set, respectively. (b) Plot of drift time of PC cations spanning mass range $700-800 \mathrm{Da}$ in traveling wave ion mobility spectrometer versus ion mass. Experimentally determined data for saturated PC and unsaturated PC cations are shown as solid squares and empty circles, respectively. The black dash and red solid lines are the linear fit to the saturated PC cation data set and to both saturated and unsaturated PC cation data set, respectively. (c) Plot of drift time of protonated and sodiated PC cations in traveling wave ion mobility spectrometer versus ion mass. Experimentally determined data for protonated PC and sodiated PC cations are shown as solid squares and empty circles, respectively. The black dash and red solid lines are the linear fit to the protonated PC cation data set and to both protonated and sodiated PC cation data set, respectively. 
Table 6.3: Collision Cross-Sections of Phosphatidylcholine Cations in $\mathrm{N}_{2}$ Drift Gas Estimated and Evaluated using Empiric Calibration Method and Equations from Shavartsburg and Smith [27], respectively. Theoretically Determined Collision Cross-Sections in $\mathrm{N}_{2}$ and He are also Listed.

\begin{tabular}{ccccccc}
\hline \hline & & \multicolumn{5}{c}{$\Omega_{D}\left(\AA^{2}\right)$} \\
\cline { 3 - 7 } PC & mass & $\begin{array}{c}\text { Estimated } \\
\text { (linear fit) }\end{array}$ & $\begin{array}{c}\text { Estimated } \\
\text { (power fit) }\end{array}$ & Evaluated & $\begin{array}{c}{ }^{a} \text { Theoretical } \\
\left.\text { (in } \mathrm{N}_{2}\right)\end{array}$ & $\begin{array}{c}{ }^{a} \text { Theoretical } \\
\text { (in He) }\end{array}$ \\
\hline 5:0-5:0 & 426 & 143.4 & 136.1 & 244.8 & 255.3 & 162.5 \\
8:0-8:0 & 510 & 171.0 & 159.5 & 289.2 & 301.3 & 197.3 \\
11:0-11:0 & 594 & 193.1 & 183.0 & 327.3 & 335.0 & 223.8 \\
14:0-16:0 & 706 & 214.9 & 210.7 & 367.0 & 361.1 & 248.2 \\
16:0-16:0 & 734 & 221.9 & 220.4 & 379.9 & 400.7 & 277.1 \\
18:0-14:0 & 734 & 221.1 & 219.3 & 378.4 & 363.7 & 254.4 \\
16:0-18:2 & 758 & 220.1 & 218.0 & 376.7 & 388.2 & 265.8 \\
$18: 0-16: 0$ & 762 & 225.5 & 225.7 & 386.7 & 364.1 & 254.2 \\
$16: 0-20: 4$ & 782 & 220.7 & 218.9 & 377.8 & 357.0 & 250.6 \\
18:0-18:2 & 786 & 224.5 & 224.4 & 385.0 & 387.1 & 270.3 \\
18:0-18:1 & 788 & 225.3 & 225.5 & 386.4 & 396.2 & 274.4 \\
18:0-18:0 & 790 & 230.5 & 233.2 & 396.2 & 395.3 & 275.3 \\
16:0-22:6 & 806 & 223.6 & 223.2 & 383.3 & 366.8 & 258.0 \\
$24: 0-24: 0$ & 958 & 258.7 & 280.4 & 451.2 & 423.4 & 305.1 \\
\hline \hline
\end{tabular}

${ }^{a}$ Averaged over 200 conformations.

specific peptide structures in the gas phase through Columbic interactions with backbone amide, carboxyl, amine, and functional groups [35, 36]. In contrast, PC is composed of two esterified acyl chains and one phosphorylcholine attached to glycerol [1]. In the sodiated PC cations, sodium cation interacts solely with the phosphate group without inducing a noticeable conformation change of PC. Thus, a good correlation between mass and mobility is observed from PC cations regardless of whether they are protonated or sodiated.

\subsubsection{Estimated Collision Cross-Sections of Ions Using T-Wave Calibra- tion}

A number of studies have employed empiric calibration methods to estimate collision cross-sections of ions using a set of calibrant ions $[17,21,22,25,26]$. To understand the structural characteristics related to collision cross-sections of $\mathrm{PC}$ cations, the calibration method was applied to estimate collision cross-sections. Figure 6.2 a shows the calibration plots of $\Omega_{D}^{\prime}$ versus $t_{d}^{\prime \prime}$ for 14 singly charged peptides and $6 \mathrm{PC}$ cations (Table 6.1). Due to the different natures of peptide and PC ions in the gas phase, we fit only the peptide calibrants first. Then we compared the fit of peptide calibrants to the fit result from the combined peptide and PC calibrants. Both linear fit and power fit to the calibrants were performed, and both fittings exhibit a high correlation coefficient $\left(R^{2}=0.98\right.$ and 0.99, respectively). Thalassinos et al. reported that linear fit is appropriate for calibration with 
small peptides. However, a slightly higher correlation was observed for power fitting in the present study. Nearly identical calibration curves were obtained from both fits for peptide and combined peptide and PC calibrants. The nature of ions in the gas phase influenced the different mass-mobility correlations. However, the empiric calibration considered only the relationship between $\Omega_{D}^{\prime}$ and $t_{d}^{\prime \prime}$. Thus, utilizing appropriate $\Omega_{D}$ for calibration is more important than the chemical category of the calibrant. Figure $6.2 \mathrm{~b}$ summarizes the estimated collision cross-sections of protonated PC cations. The estimated collision cross-section values of PC cations are found in Table 6.3.

\subsubsection{Determination of Collision Cross-Sections of Ions}

Shavartsburg and Smith derived equations to describe the quantitative relationship between drift time and ion mobility in TWIMS [27]. Under the condition that $K E_{\max }<s$, where $K$ is ion mobility, $E_{\max }$ is maximum electric field $(E)$, and $s$ is wave velocity, the mobility of an ion is related to the average ion velocity in TWIMS as [27]

$$
\bar{v}=\frac{K^{2}}{b s} \int_{0}^{b} E^{2}(x) d s,
$$

where $E(x)$ is a half-sinusoidal traveling wave function and $b$ is the waveform baseline width. Note that the equation ignores the focusing field and restricts the dynamics to axial coordinates of the SRIG. The rearrangement of Equation 6.1 with drift length $L$ and the corrected drift time $t_{d}^{\prime}$ yields

$$
K=\sqrt{\frac{L b s}{t_{d}^{\prime} \int_{0}^{b} E^{2}(x) d x}} .
$$

Once $K$ is determined, the reduced mobility $K_{0}$ can be determined according to

$$
K_{0}=\left(\frac{273 \mathrm{~K}}{T}\right)\left(\frac{P}{760 \text { Torr }}\right) K,
$$

where $P$ and $T$ are the experimental pressure and temperature, respectively. Finally, the collision cross-section of an ion is evaluated by the relation $[37,38]$

$$
\Omega_{D}=\frac{3 q}{16 N_{0}}\left(\frac{2 \pi}{\mu_{N_{2}} k_{B} T}\right)^{1 / 2} \frac{1}{K_{0}},
$$

where $N_{0}$ is the number density at standard state (273 K and 760 Torr), $q$ is the charge on the ion, $\mu$ is the reduced mass of ion and $\mathrm{N}_{2}, k_{B}$ is the Boltzmann constant, $T$ is the temperature in the drift region, and $\Omega_{D}$ is the collision cross-section. The evaluated collision cross-sections of the examined PC cations are listed in Table 6.3. Note that a significant difference is found between the estimated $\Omega_{D}$ and the evaluated $\Omega_{D}$. The evaluated $\Omega_{D}$ values are on average $\sim 42 \%$ larger than the estimated $\Omega_{D}$ values from both power and linear fit. It is of note that the collision cross-sections of calibrants 


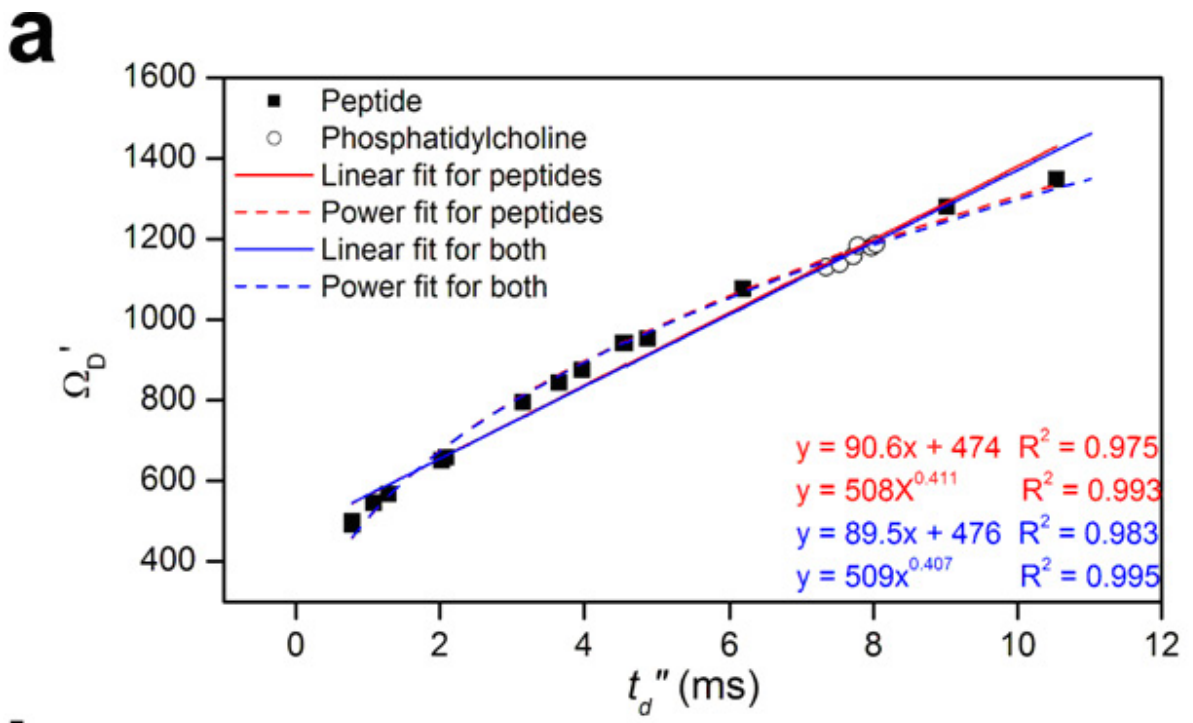

b

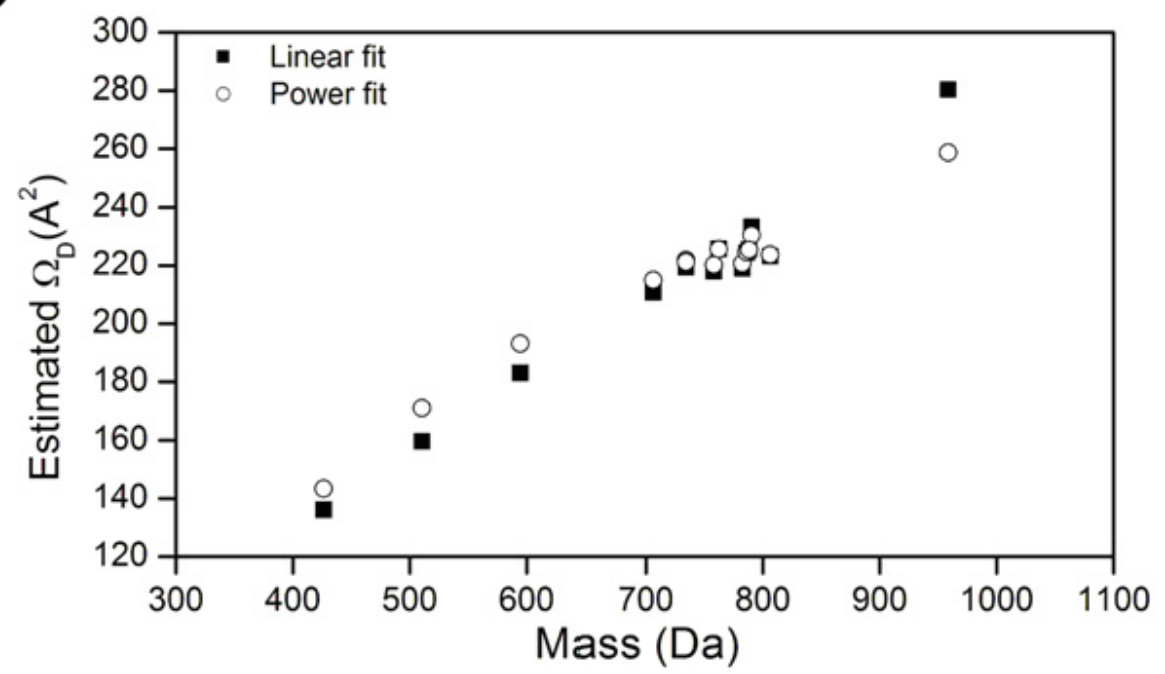

Figure 6.2: (a) Plot of corrected empiric cross-sections versus effective drift times for 14 peptides and 4 phosphatidylcholines (PC). For each peptide and PC the singly charged cation is used. Linear trend and power trend lines are shown as solid and dash lines, respectively. (b) A plot of the estimated cross-sections versus the ion mass for PC cations investigated in this study. The estimated collision cross-sections from linear trend and power trend are shown as solid squares and empty circles, respectively. 
are determined in He $[9,16]$ while the drift gas used in TWIMS is $\mathrm{N}_{2}$. A strong contribution of short range interaction between ion and neutral is expected for $\Omega_{D}$ of an ion at the mass range of PC [20]. Yet, a considerable contribution is still considered from long range interactions of ion-neutral, linear shape, and larger mass in $N_{2}$ for the determination of $\Omega_{D}$ of an ion. It is inferred that the observed difference of $\Omega_{D}$ values are caused by lack of these terms in the calibration procedure.

\subsubsection{Calculated Collision Cross-Sections of Ions Using the Trajectory Method}

The $\Omega_{D}$ of the PC cations investigated in this study were calculated using the TJ method in $\mathrm{N}_{2}$ [20] and He (Table 6.3) [28]. The MD simulation trajectories of the PC cation for 200 ps reveal that the two acyl chains undergo large structural fluctuation due to the thermal energy at 300 K. Figure 6.3a shows the time profile of the C-C distance between the carbon atoms at the end of each chain of the 18:0-18:0 PC during 200 ps of dynamics. The distance between two carbon atoms fluctuates in the range of 5 to $25 \AA$ within a 5 to 20 ps time period. In order to account for the sufficient amount of conformational change required for $\Omega_{D}$ calculation, we need to sample the conformations at every 1 ps. Then, the average $\Omega_{D}$ can be determined using the TJ method in $\mathrm{N}_{2}$ and He for the 200 structures on the MD simulation trajectories [20, 28].

Figure 6.3b shows the plot of $\Omega_{D}$ for PC cations evaluated using the Shavartsburg and Smith [27] equations versus the theoretical $\Omega_{D}$ in $\mathrm{N}_{2}$ calculated using the modified TJ method. The theoretical $\Omega_{D}$ values of PC cations exhibit good agreement with the experimentally evaluated values. The agreement is within $6.2 \%$ in the worst-case deviation with $3.2 \%$ deviation on average. This shows that the experimental collision cross-sections of analyte ions can be determined using Synapt HDMS and the relationship between SRIG drift time and mobility derived by Shavartsburg and Smith [27]. In contrast, poor agreement was observed from the estimated $\Omega_{D}$ of PC cations from the linear fit and power fit calibration curves with deviations of $71.2 \%$ and $73 \%$ on average, respectively.

\subsection{Discussion}

\subsubsection{Effect of Drift Gas on Ion Mobility}

The difference between the estimated $\Omega_{D}$ of the PC cation using empiric calibration and the evaluated $\Omega_{D}$ using Equations 6.2 to 6.4 can be explained by the different polarizabilities, sizes, and shapes of $\mathrm{He}$ and $\mathrm{N}_{2}$ molecules. In drift tube ion mobility spectrometry (DTIMS), the drift time, which corresponds to the effective drift time in TWIMS, $t_{d}^{\prime \prime}$, is inversely proportional to the ion mobility, $K$ : 

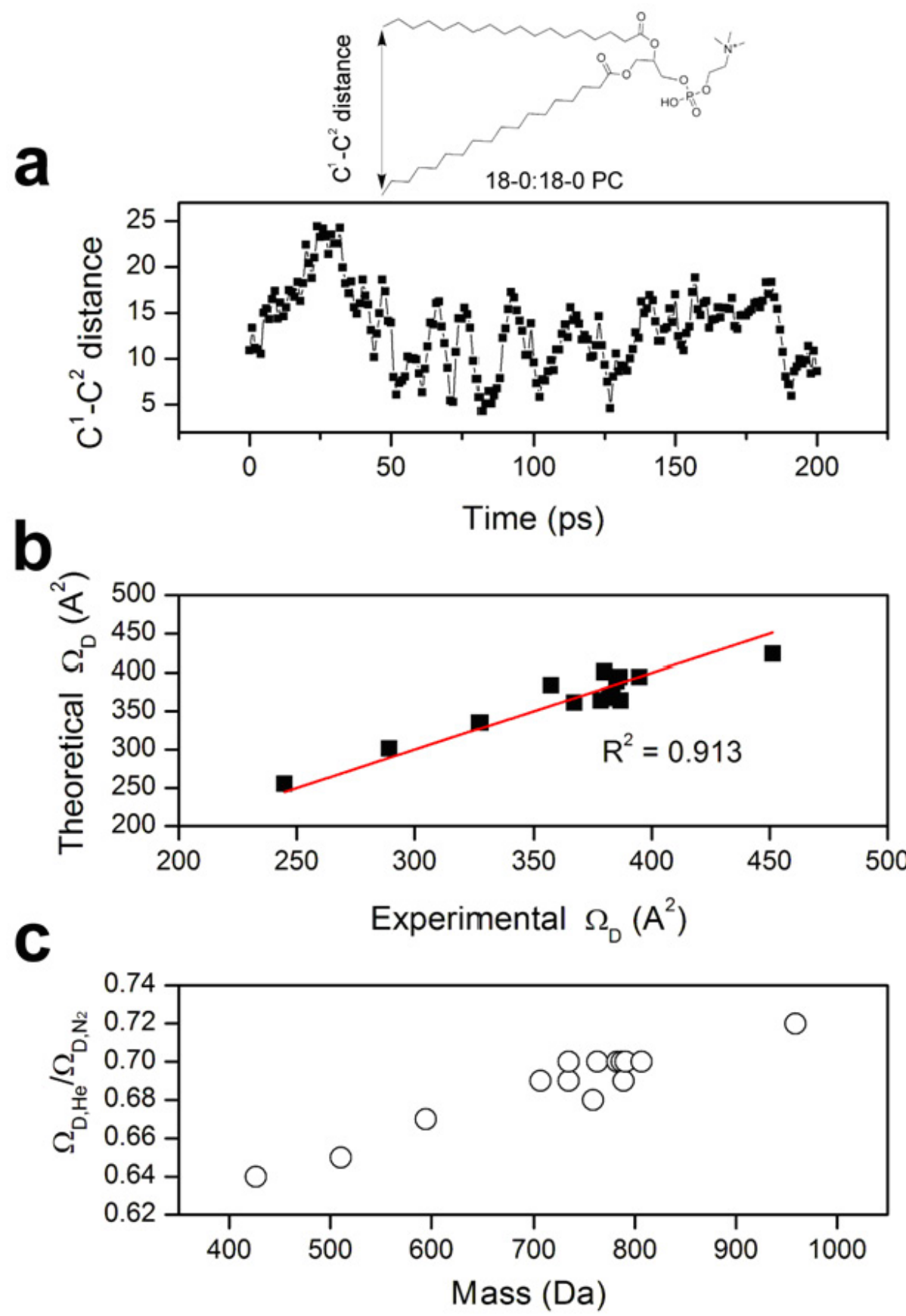

Figure 6.3: (a) Time profile of the distance between the carbon atoms at the end of each acyl chain of 18:0-18:0 phophatidylcholine during $200 \mathrm{ps}$ of the molecular dynamics simulation. The fluctuation is ranging from $\sim 5 \AA$ to $\sim 25 \AA$ with the time period of $5-20$ ps. Approximately 17 times of fluctuation is observed from this trajectory. (b) Plot of experimentally determined collision crosssections $\left(\Omega_{D}\right)$ of phosphatidylcholine (PC) cations in $\mathrm{N}_{2}$ against theoretically determined $\Omega_{D}$ using the modified TJ method for $\mathrm{N}_{2}$ drift gas. The theoretical $\Omega_{D}$ is obtained by averaging $\Omega_{D}$ for 200 structures from MD simulations. The solid line is $y=x$. (c) Plot of theoretical $\Omega_{D}$ in He over theoretical $\Omega_{D}$ in $\mathrm{N}_{2}$ versus mass of PC cations. 


$$
K=L^{2} / V t_{d}^{\prime \prime}
$$

where $V$ is voltage across the drift tube. The relationship between $K$ and $\Omega_{D}$ is described as [39]

$$
K=\frac{3 q}{16 N}\left(\frac{2 \pi}{\mu k_{B} T}\right)^{1 / 2} \frac{1}{\Omega_{D}} .
$$

The corrected collision cross-section, $\Omega_{D}^{\prime}$, for the empiric calibration is defined as $[17,26]$

$$
\Omega_{D}^{\prime}=\frac{\Omega_{D} \mu^{1 / 2}}{q} .
$$

From Equations 6.5 through 6.7, we obtain the proportional relationship between $t_{d}^{\prime \prime}$ and $\Omega_{D}^{\prime}$. As discussed earlier, the default drift gas of TWIMS of Synapt HDMS is $\mathrm{N}_{2}[17,30,40]$. Calibration methods commonly employ the empirical $\Omega_{D}$ determined in He [16]. Thus, the corrected collision cross-section, $\Omega_{D, N_{2}}^{\prime}$, using the reduced mass in $\mathrm{N}_{2}, \mu_{N_{2}}$, is related to the $\Omega_{D}$ in He as

$$
\Omega_{D, N_{2}}^{\prime}=\frac{\Omega_{D, N_{2}} \mu_{N_{2}}^{1 / 2}}{q}=\frac{\Omega_{D, H e} \mu_{N_{2}}^{1 / 2}}{q} .
$$

This relationship works if $\Omega_{D, H e} \approx \Omega_{D, N_{2}}$. Hill and co-workers have demonstrated the high dependence of $\Omega_{D}$ of ions on drift gas [41, 42]. Beegle et al. demonstrated the different polarizability effects of the drift gas molecule on the $\Omega_{D}$ of the ion molecule using a series of homologous Gly peptides [41]. As the size of the Gly peptide increases, the difference between $\Omega_{D}$ in $\mathrm{N}_{2}$ and in $\mathrm{He}$ decreases. The short range interaction for $\Omega_{D}$ becomes more important as the ion size increases [20]. Thus, the pre-assumption for the empiric calibration is valid when the size of the ion is very large; geometric factors of neutral and long range ion-neutral interactions are completely negligible for the determination of $\Omega_{D}$ [26]. Theoretically calculated $\Omega_{D}$ in $\mathrm{N}_{2}$ and He further support this argument. Figure 6.3c shows the plot of the theoretical $\Omega_{D}$ in He divided by $\Omega_{D}$ in $\mathrm{N}_{2}$ versus the mass of PC cations. As the size of the cation increases from $426 \mathrm{Da}$ to $959 \mathrm{Da}$, the agreement between the two theoretical $\Omega_{D}$ values increases from $64 \%$ to $72 \%$. As a result, for the mass range of the PC cations (400 - $1000 \mathrm{Da}$ ), estimating $\Omega_{D}$ using the empiric calibration method is not valid.

\subsubsection{Geometrical Effect on the Collision Cross-Sections of Phosphatidyl- choline Cations}

Figure 6.4a shows the plot of theoretical characteristic $\Omega_{D}$ of PC cations versus ion mass compared with the corresponding surface area of PC cations in $\mathrm{N}_{2}$ at $300 \mathrm{~K}$ using the Maximal Speed Molecular Surface (MSMS) program [43, 44]. Note that high similarity is observed from the characteristics of relative $\Omega_{D}$ from theoretical calculation and the relative surface areas of PC cations. This implies 
that the $\Omega_{D}$ for each PC cation is largely influenced by the short range van der Waals interaction between the ion and the neutral $\mathrm{N}_{2}$ molecule. The molecular weight and specific geometry of the ions dominate the short range van der Waals interaction, which affects the collision cross-section of the ion $[20]$.

The mobility of ion $K$ becomes field-dependent at a high electric field [45]. The field dependence of $K$ depends on the nature properties of ion-neutral interactions. In general, high field behavior of an ion is observed when the ion acquires enough energy from $E$ to change the nature of the ion-neutral collisions [45]. The total average energy of the ions can be determined from the Wannier energy formula as follows:

$$
\frac{3}{2} k_{B} T_{e f f}=\frac{3}{2} k_{B} T+\frac{1}{2} M v_{d}^{2}
$$

where $T_{\text {eff }}$ is an effective temperature of ion, $M$ is the mass of a drift gas molecule, and $v_{d}$ is the drift velocity of an ion [46]. The thermal kinetic energy is $3 / 2 k_{B} T$, and the field energy is $1 / 2 M v_{d}^{2}$. The low field behavior of an ion is achieved when

$$
\frac{3}{2} k_{B} T \gg \frac{1}{2} M v_{d}^{2}
$$

Ion mobility spectrometers typically operate at low electric fields. The typical $E / N$ range for the low field is a few Townsend $\left(\mathrm{Td}=10^{-17} \mathrm{Vcm}^{2}\right)[18,45]$. Although the applied voltage in the TWIMS is as low as $8 \mathrm{~V}$ in this study, due to the low pressure of the SRIG (0.39 Torr in this study), the average $E / N$ is $\sim 80 \mathrm{Td}$. This is an order of magnitude larger than common IMS operating field. In addition, $E / N$ increases to as much as $\sim 230 \mathrm{Td}$ at $E_{\max }$ of traveling wave.

The primary effect of a high electric field is to heat the ions [45], which increases their internal energy through ion-neutral collisions. This collisional activation can result in conformation changes of the ions. Figure $6.4 \mathrm{~b}$ shows the plots of the $\Omega_{D}$ of PC cations evaluated using the Shavartsburg and Smith [27] equations versus ion mass compared with the corresponding surface area of PC cations in $\mathrm{N}_{2}$ at $300 \mathrm{~K}$ and $400 \mathrm{~K}$. Greater similarity is observed from the characteristic relative $\Omega_{D}$ and the relative surface areas of PC cations at $400 \mathrm{~K}$ compared to the relative surface areas at $300 \mathrm{~K}$. Although the experiment was performed at $\sim 300 \mathrm{~K}$, field heating induced a shift in ion conformation distribution to slightly higher energy state. This results in a greater similarity of the characteristic between relative $\Omega_{D}$ at $300 \mathrm{~K}$ and relative surface area at higher temperature, $400 \mathrm{~K}$.

\subsubsection{Mass-Mobility Correlations of Phophatidylcholine Cations}

Saturated PC cations investigated in this study exhibit a good correlation between mass and mobility (Figure 6.1a). However, deviations from the correlation are observed in unsaturated PC cations

(Figure 6.1b). In the previous study, we discussed the importance of van der Waals potential for 


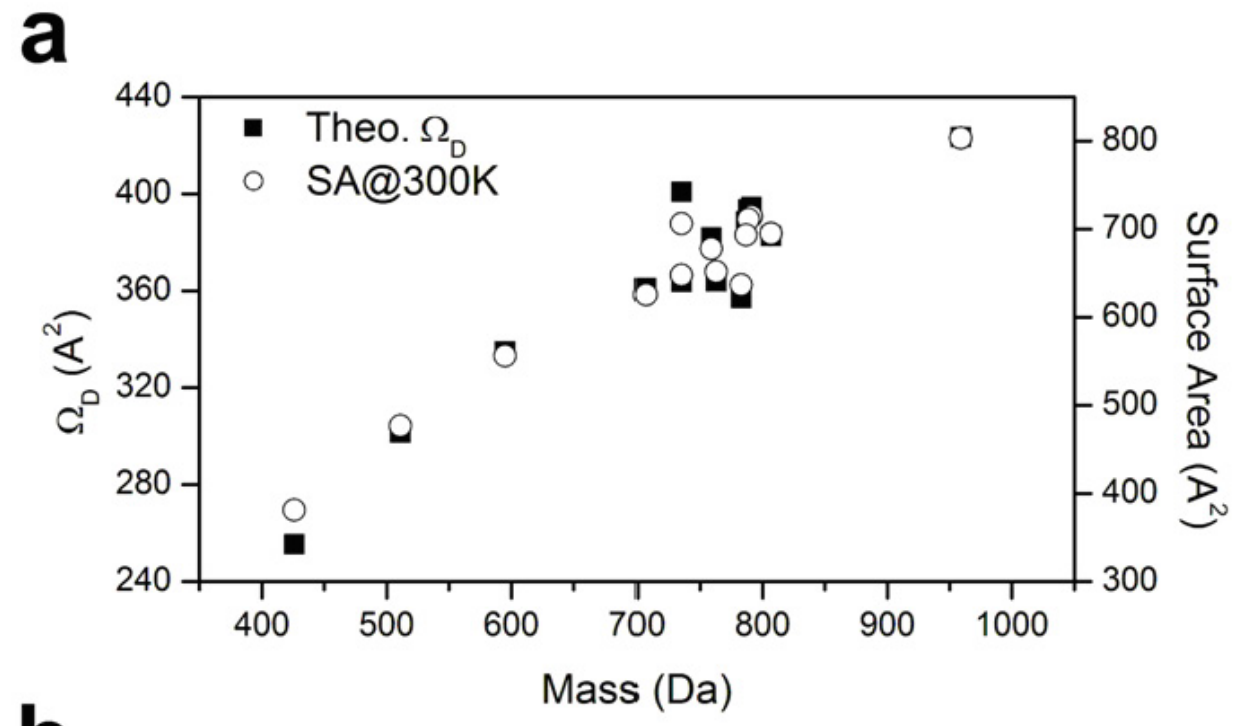

b

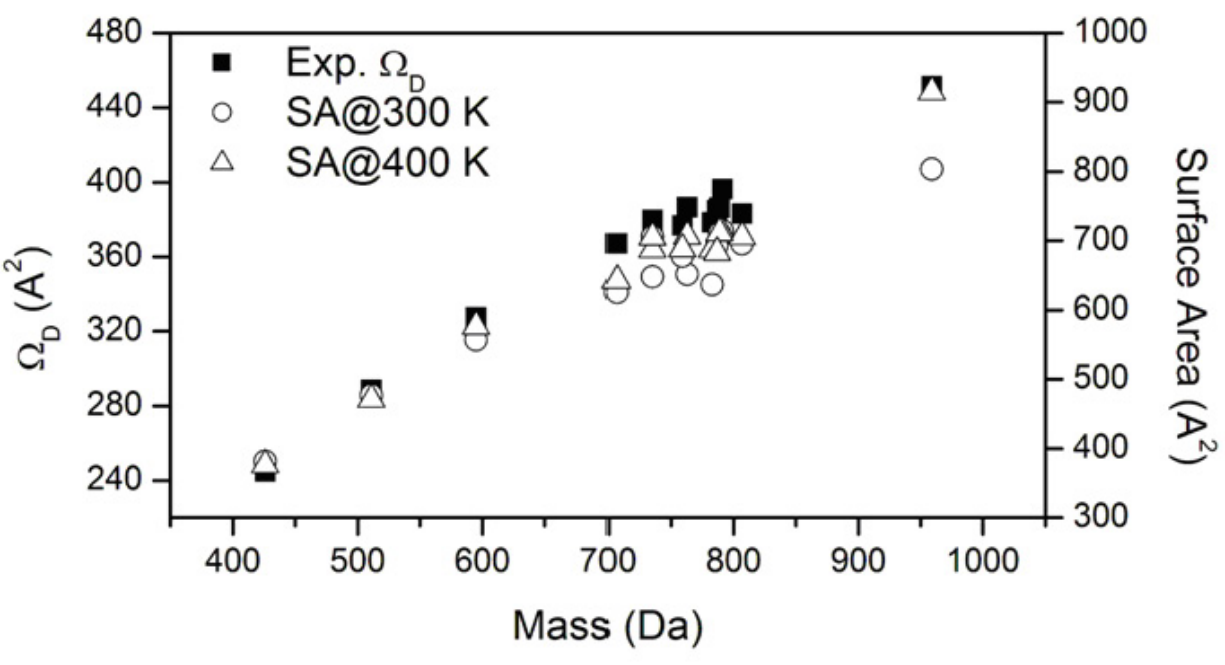

Figure 6.4: (a) Plots of theoretically determined collision cross-sections $\left(\Omega_{D}\right)$ and surface areas of phosphatidylcholine (PC) cations in $\mathrm{N}_{2}$ versus ion mass. The calculated average $\Omega_{D}$ of the 200 ion conformations are shown as solid squares (left $y$-axis). The calculated surface areas of PC cations in $\mathrm{N}_{2}$ at $300 \mathrm{~K}$ are shown as empty circles (right $y$-axis). (b) Plots of experimentally evaluated $\Omega_{D}$ and surface areas of phosphatidylcholine (PC) cations in $\mathrm{N}_{2}$ versus ion mass. The $\Omega_{D}$ of PC cations are shown as solid squares (left $y$-axis). The calculated surface areas of $\mathrm{PC}$ cations in $\mathrm{N}_{2}$ at $300 \mathrm{~K}$ and $400 \mathrm{~K}$ are shown as empty circles and empty triangle, respectively (right $y$-axis). 
determining the collision cross-section of an ion as the ion size increases [20]. This implies that strong mass-mobility correlation is highly affected by the geometry of the ion. In order to understand the mass-mobility correlation of saturated PC cations and deviations of unsaturated PC cations from the correlation plot, we investigate structures of PC cations with corresponding $\Omega_{D}$.

The minimum energy $\left(E_{0}\right)$ structures of some of the saturated PC cations examined in this study are shown in Figure 6.5a. The structures with the closest $\Omega_{D}$ to the experimental values are also shown along with their corresponding relative energy values $\left(E^{*}\right)$. The plot of $\Omega_{D}$ for the PC cations experimentally evaluated using the Shavartsburg and Smith [27] equations versus the theoretical $\Omega_{D}$ values of $E_{0}$ and $E^{*}$ is shown in Figure $6.5 \mathrm{~b}$. Slightly larger $\Omega_{D}$ values are observed from PC cations with longer acyl chains ( $\geq 18$ carbon) compared to the $\Omega_{D}$ values calculated from minimum energy structures. In contrast, smaller $\Omega_{D}$ values are observed from PC cations with short acyl chains $(\leq 16$ carbon). Note that PC cations with an acyl chain longer than 16 carbons form globular structures that are energetically favored. It is inferred that intramolecular van der Walls interactions of acyl chains drives the globular conformation to be preferred for large PC cations. However, extended structures are energetically favored for those with a shorter acyl chain (fewer than 16 carbons), whose steric effects prevent them from forming globular conformations in the gas phase. In contrast to peptide or protein ions, PC cations do not have strong intramolecular interactions to stabilize certain conformations. The energy difference between the $E_{0}$ structure and the $E^{*}$ structure is only $19 \mathrm{kcal} / \mathrm{mol}$ on average. Thus, the conformations of these ions may fluctuate while traveling in the SRIG. As discussed earlier, the internal energy of an ion increases with collisional activations from the traveling wave electric field, which results in continual excitation of the ion [27]. The shift in ion conformations occurs toward slightly excited $\left(E^{*}\right)$ state. Especially for larger molecules (18 or more carbon acyl chains), a significant increase in $\Omega_{D}$ occurs, since the increase in internal energy of these large molecules increases the importance of the entropy. As a result, the structural similarity of the saturated PC cations is maintained with moderately extended structures regardless of the length and symmetry of the PC acyl chains (Figure 6.5a); this promotes a good correlation between mass and mobility.

\subsubsection{Characterizing Unsaturated Phosphatidylcholines from Mass-Mobility Correlation}

Unsaturated PC cations exhibit significantly deviated mobility values from the mass-mobility correlation plot of saturated PC cations (Figure 6.1b). The drift time is reduced by $\sim 5 \%$ for the unsaturated PC cation with one double bond; the drift time reduces further at a rate of $\sim 1 \%$ for the additional double bond. Jackson et al. recently reported a $\sim 0.5 \%$ reduction in drift time for each additional double bond of phospholipids in DTIMS. In the present study, the larger difference in 
a

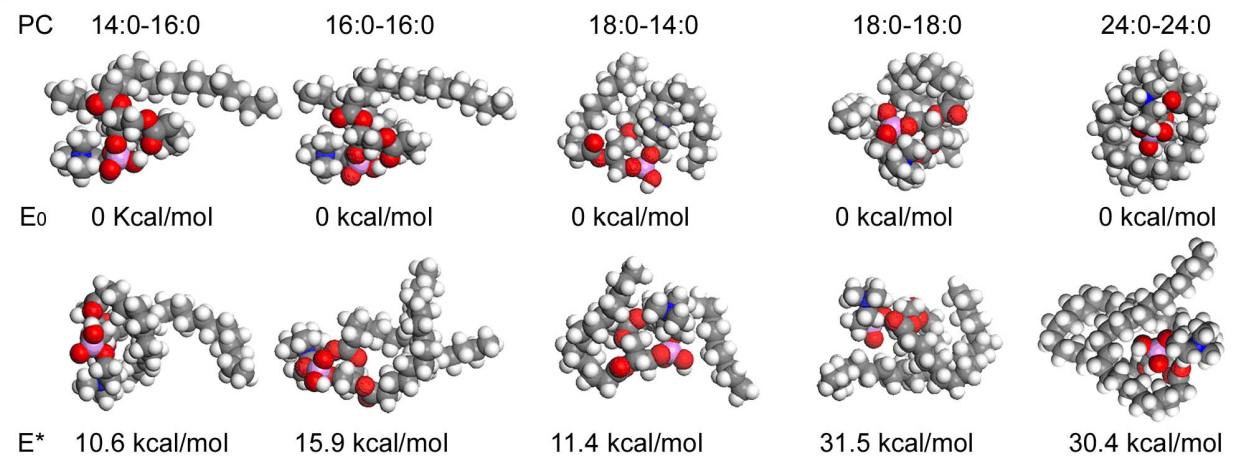

b

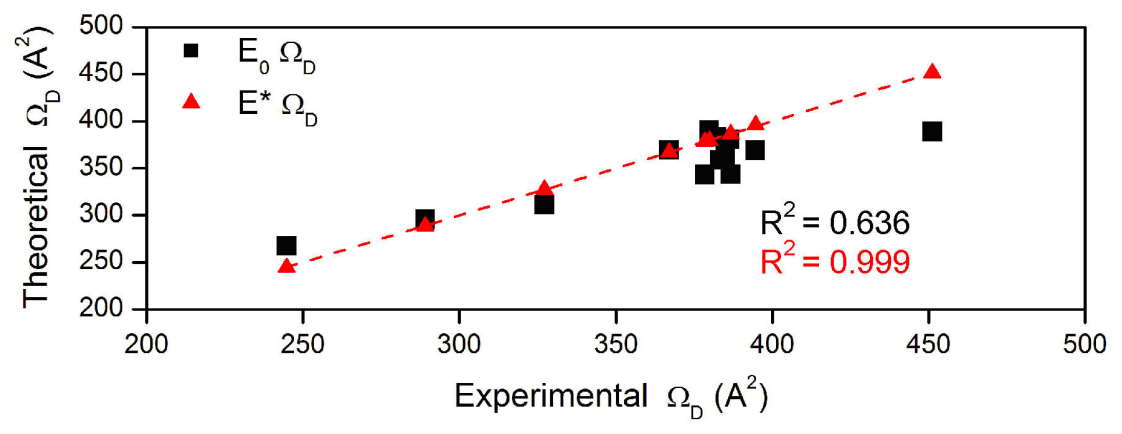

Figure 6.5: (a) MD simulated structures of saturated phosphatidylcholine cations at minimum energy state $\left(E_{0}\right)$ are shown. The structures of the closest $\Omega_{D}$ to the experimental values are also shown along with corresponding relative energy values $\left(E^{*}\right)$. (b) Plot of experimentally determined collision cross-sections $\left(\Omega_{D}\right)$ of phosphatidylcholine (PC) cations in $\mathrm{N}_{2}$ against theoretically determined $\Omega_{D}$ at $E_{0}$ and $E^{*}$ using the modified TJ method for $\mathrm{N}_{2}$ drift gas. The solid line is $y=x$. 
Table 6.4: Theoretically Determined Collision Cross-Sections $\left(\Omega_{D}\right)$ of Phosphatidylcholine Cations at Minimum Energy State $\left(E_{0}\right)$. The Differences of $\Omega_{D}\left(\Delta \Omega_{D}\right)$ and Potential Energy $(\Delta E)$ from the PC Structure at $E_{0}$ to Experimentally Determined $\Omega_{D}$.

\begin{tabular}{cccc}
\hline \hline $\mathrm{PC}$ & $\Omega_{D, E_{0}}\left(\AA^{2}\right)$ & $\Delta \Omega_{D}(\%)$ & $\Delta E(\mathrm{kcal} / \mathrm{mol})$ \\
\hline 5:0-5:0 & 267.08 & 9.1 & 15.2 \\
8:0-8:0 & 295.44 & 2.1 & 10.6 \\
11:0-11:0 & 311.24 & -4.9 & 34.7 \\
14:0-16:0 & 369.21 & 0.6 & 10.3 \\
16:0-16:0 & 390.55 & 2.8 & 15.9 \\
18:0-14:0 & 343.25 & -9.3 & 11.4 \\
16:0-18:2 & 382.85 & 1.6 & 10.4 \\
18:0-16:0 & 343.38 & -11.2 & 28.7 \\
16:0-20:4 & 333.7 & -11.7 & 26.8 \\
18:0-18:2 & 362.37 & -5.9 & 10.6 \\
18:0-18:1 & 380.32 & -1.6 & 2.21 \\
18:0-18:0 & 368.93 & -6.9 & 31.5 \\
16:0-22:6 & 358.53 & -6.5 & 30.2 \\
$24: 0-24: 0$ & 388.94 & -13.8 & 30.4 \\
\hline \hline
\end{tabular}

the mobility of unsaturated PC cations compared to saturated PC cations results from the different rate of conformation changes in TWIMS. Figure 6.6 shows structures of selected unsaturated PC cations at $E_{0}$ along with the conformations at $E^{*}$. For unsaturated $\mathrm{PC}$ cations, a smaller shift in $\Omega_{D}$ is observed from the $\Omega_{D}$ of the most stable conformation compared to saturated PCs of similar mass. For those PC cations with more than 16 carbon acyl chains, saturated PC cations exhibit $\mathrm{a} \sim 10 \%$ difference in $\Omega_{D}$ on average, while unsaturated PC cations show only $\sim 5 \%$ difference on average (Table 6.4). As observed in Figure 6.6, the major change in conformation occurs at the saturated acyl chain, while the conformation of the unsaturated acyl chain (yellow) maintains a bent structure. The presence of cis-double bonds in an acyl chain prevents the unsaturated acyl chain from extending by activation. As a result, less fluctuation in the ion structure occurred among unsaturated PC cations in the TWIMS. Unsaturated PC cations show smaller $\Omega_{D}$ values than saturated PC cations, which can form more extended conformations. This is logical given that globular structures of unsaturated PC cations are more compact and therefore have smaller collision crosssections. This allows us to characterize unsaturated PC cations based on their mobility, and thus collision cross-sections, using TWIMS.

\subsection{Conclusions}

A high correlation between mass and mobility in $\mathrm{N}_{2}$ is observed from a number of saturated PC cations in TWIMS. A significant deviation from this mass-mobility correlation is observed with unsaturated PC cations. Theoretical investigation using a modified TJ method indicates that the 
16:0-18:2 PC

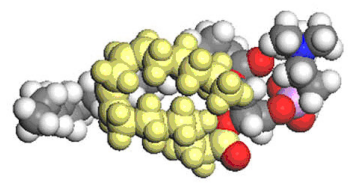

$\mathrm{E}_{0}=0 \mathrm{kcal} / \mathrm{mol}$ $\Omega_{\mathrm{D}}=382.9 \AA^{2}$

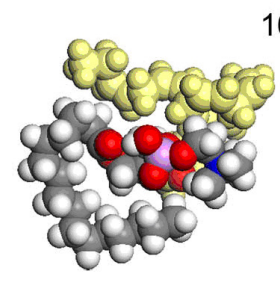

$\mathrm{E}_{0}=0 \mathrm{kcal} / \mathrm{mol}$ $\Omega_{\mathrm{D}}=358.5 \AA^{2}$

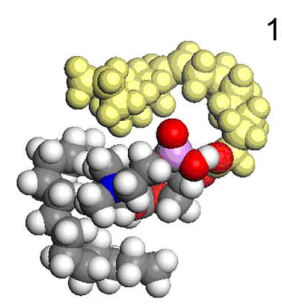

$\mathrm{E}_{0}=0 \mathrm{kcal} / \mathrm{mol}$ $\Omega_{\mathrm{D}}=380.3 \AA^{2}$

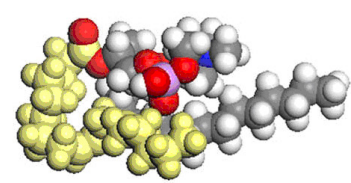

$E^{*}=10.4 \mathrm{kcal} / \mathrm{mol}$ $\Omega_{\mathrm{D}}=376.7 \AA^{2}$

16:0-22:6 PC

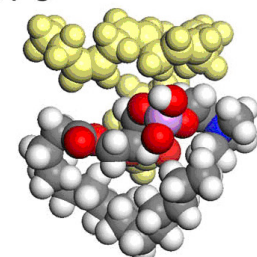

$E^{*}=30.2 \mathrm{kcal} / \mathrm{mol}$ $\Omega_{\mathrm{D}}=383.1 \AA^{2}$

18:0-18:1 PC

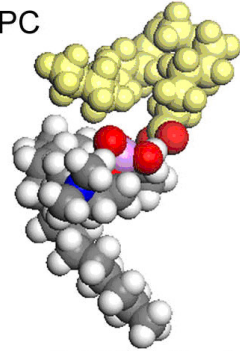

$E^{*}=2.21 \mathrm{kcal} / \mathrm{mol}$ $\Omega_{\mathrm{D}}=386.2 \AA^{2}$

Figure 6.6: MD simulated structures of unsaturated phosphatidylcholine cations at minimum energy state $\left(E_{0}\right)$ are shown. The structures of the closest $\Omega_{D}$ to the experimental values are also shown along with corresponding relative energy values $\left(E^{*}\right)$. Unsaturated acyl chain is colored in yellow. 
empiric calibration method is not suitable to estimate collision cross-sections for PC cations. Instead, we evaluate collision cross-sections using a quantitative relationship between drift time and mobility derived by Shavartsburg and Smith [27]. In addition to the lack of intramolecular interactions among PC cations, collisional excitation of the ions in the SRIG induces a shift in ion conformational distribution. The unsaturated acyl chain remains bent, while the saturated acyl chain extends under the electric field, which causes larger collision cross-sections for saturated PCs and smaller ones for unsaturated PCs. The initial double bond in the acyl chain yields an approximately $5 \%$ reduction in drift time, with further drift time reduction at the rate of $\sim 1 \%$ for each additional double bond. As a result, greater separation and characterization of unsaturated PC cations can be achieved using TWIMS.

\subsection{Acknowledgments}

This research was carried out at the Jet Propulsion Laboratory, California Institute of Technology, under a contract with the National Aeronautics and Space Administration (NASA), the University of California Los Angeles Mass Spectrometry and Proteomics Technology Center, and the Material and Process Simulation Center, Beckman Institute, California Institute of Technology. Financial support through NASA's Astrobiology Science and Technology Instrument Development, Planetary Instrument Definition and Development, and Mars Instrument Development programs is gratefully acknowledged. JAL acknowledges support from the NIH (RR20004). 


\section{Bibliography}

[1] Gurr, M. I.; Hardwood, J. L. Lipid Biochemistry; 4th ed.; Chapman and Hall: New York, 1991.

[2] Simoneit, B. R. T.; Summons, R. E.; Jahnke, L. L. Orig. Life Evol. Biosph. 1998, 28, 475-483.

[3] Mossoba, M. M.; Kramer, J. K. G.; Brenna, J. T.; McDonald, R. E. Lipid Analysis and Lipidomics AOCS Publishing 2006.

[4] Woods, A. S.; Ugarov, M.; Egan, T.; Koomen, J.; Gillig, K. J.; Fuhrer, K.; Gonin, M.; Schultz, J. A. Anal. Chem. 2004, 76, 2187-2195.

[5] Jackson, S. N.; Ugarov, M.; Egan, T.; Post, J. D.; Langlais, D.; Schultz, J. A.; Woods, A. S. J. Mass Spectrom. 2007, 42, 1093-1098.

[6] Jackson, S. N.; Ugarov, M.; Post, J. D.; Egan, T.; Langlais, D.; Schultz, J. A.; Woods, A. S. J. Am. Soc. Mass Spectrom. 2008, 19, 1655-1662.

[7] McLean, J. A.; Ridenour, W. B.; Caprioli, R. M. J. Mass Spectrom. 2007, 42, 1099-1105.

[8] Fenn, L. S.; McLean, J. A.; Springer Heidelberg: 2008, p 905-909.

[9] Fenn, L. S.; Kliman, M.; Mahsut, A.; Zhao, S. R.; McLean, J. A. 2009, In Press.

[10] Gidden, J.; Bowers, M. T. J. Am. Soc. Mass Spectrom. 2003, 14, 161-170.

[11] Fenn, L. S.; McLean, J. A. Chem. Commun. 2008, 5505-5507.

[12] Jarrold, M. F. Annu. Rev. Phys. Chem. 2000, 51, 179-207.

[13] Griffin, G. W.; Dzidic, I.; Carroll, D. I.; Stillwel, R. N.; Horning, E. C. Anal. Chem. 1973, 45, 1204-1209.

[14] Berant, Z.; Karpas, Z. J. Am. Chem. Soc. 1989, 111, 3819-3824.

[15] Karpas, Z.; Berant, Z. J. Phys. Chem. 1989, 93, 3021-3025.

[16] Valentine, S. J.; Counterman, A. E.; Clemmer, D. E. J. Am. Soc. Mass Spectrom. 1999, 10, 1188-1211. 
[17] Thalassinos, K.; Grabenauer, M.; Slade, S. E.; Hilton, G. R.; Bowers, M. T.; Scrivens, J. H. Anal. Chem. 2009, 81, 248-254.

[18] Johnson, P. V.; Kim, H. I.; Beegle, L. W.; Kanik, I. J. Phys. Chem. A 2004, 108, 5785-5792.

[19] Kim, H. I.; Johnson, P. V.; Beegle, L. W.; Beauchamp, J. L.; Kanik, I. J. Phys. Chem. A 2005, 109, 7888-7895.

[20] Kim, H.; Kim, H. I.; Johnson, P. V.; Beegle, L. W.; Beauchamp, J. L.; Goddard, W. A.; Kanik, I. Anal. Chem. 2008, 80, 1928-1936.

[21] Ruotolo, B. T.; Giles, K.; Campuzano, I.; Sandercock, A. M.; Bateman, R. H.; Robinson, C. V. Science 2005, 310, 1658-1661.

[22] Ruotolo, B. T.; Hyung, S. J.; Robinson, P. M.; Giles, K.; Bateman, R. H.; Robinson, C. V. Angew. Chem.-Int. Edit. 2007, 46, 8001-8004.

[23] Smith, D. P.; Giles, K.; Bateman, R. H.; Radford, S. E.; Ashcroft, A. E. J. Am. Soc. Mass Spectrom. 2007, 18, 2180-2190.

[24] Bagal, D.; Zhang, H.; Schnier, P. D. Anal. Chem. 2008, 80, 2408-2418.

[25] Williams, J. P.; Scrivens, J. H. Rapid Commun. Mass Spectrom. 2008, 22, 187-196.

[26] Ruotolo, B. T.; Benesch, J. L. P.; Sandercock, A. M.; Hyung, S. J.; Robinson, C. V. Nat. Protoc. 2008, 3, 1139-1152.

[27] Shvartsburg, A. A.; Smith, R. D. Anal. Chem. 2008, 80, 9689-9699.

[28] Mesleh, M. F.; Hunter, J. M.; Shvartsburg, A. A.; Schatz, G. C.; Jarrold, M. F. J. Phys. Chem. 1996, 100, 16082-16086.

[29] Giles, K.; Pringle, S. D.; Worthington, K. R.; Little, D.; Wildgoose, J. L.; Bateman, R. H. Rapid Commun. Mass Spectrom. 2004, 18, 2401-2414.

[30] Pringle, S. D.; Giles, K.; Wildgoose, J. L.; Williams, J. P.; Slade, S. E.; Thalassinos, K.; Bateman, R. H.; Bowers, M. T.; Scrivens, J. H. Int. J. Mass Spectrom. 2007, 261, 1-12.

[31] MacKerell, A. D.; Bashford, D.; Bellott, M.; Dunbrack, R. L.; Evanseck, J. D.; Field, M. J.; Fischer, S.; Gao, J.; Guo, H.; Ha, S.; Joseph-McCarthy, D.; Kuchnir, L.; Kuczera, K.; Lau, F. T. K.; Mattos, C.; Michnick, S.; Ngo, T.; Nguyen, D. T.; Prodhom, B.; Reiher, W. E.; Roux, B.; Schlenkrich, M.; Smith, J. C.; Stote, R.; Straub, J.; Watanabe, M.; Wiorkiewicz-Kuczera, J.; Yin, D.; Karplus, M. J. Phys. Chem. B 1998, 102, 3586-3616.

[32] Plimpton, S. J. Comput. Phys. 1995, 117, 1-19. 
[33] Feller, S. E.; Gawrisch, K.; MacKerell, A. D. J. Am. Chem. Soc. 2002, 124, 318-326.

[34] Alberts, B.; Bray, D.; Hopkin, K.; Johnson, A.; Lewis, J.; Raff, M.; Roberts, K.; Walter, P. Essential Cell Biology; Second ed.; Garland Science: New York, 2004.

[35] Kohtani, M.; Kinnear, B. S.; Jarrold, M. F. J. Am. Chem. Soc. 2000, 122, 12377-12378.

[36] Wyttenbach, T.; Liu, D. F.; Bowers, M. T. J. Am. Chem. Soc. 2008, 130, 5993-6000.

[37] Revercomb, H. E.; Mason, E. A. Anal. Chem. 1975, 47, 970-983.

[38] Eiceman, G. A.; Karpas, Z. Ion Mobility Spectrometry; CRC Press: Boca Raton, FL, 1994.

[39] Mason, E. A.; O’hara, H.; Smith, F. J. J. Phys. B 1972, 5, 169-176.

[40] Scarff, C. A.; Thalassinos, K.; Hilton, G. R.; Scrivens, J. H. Rapid Commun. Mass Spectrom. 2008, 22, 3297-3304.

[41] Beegle, L. W.; Kanik, I.; Matz, L.; Hill, H. H. Int. J. Mass Spectrom. 2002, 216, 257-268.

[42] Matz, L. M.; Hill, H. H.; Beegle, L. W.; Kanik, I. J. Am. Soc. Mass Spectrom. 2002, 13, 300-307.

[43] Connolly, M. L. J. Am. Chem. Soc. 1985, 107, 1118-1124.

[44] Sanner, M. F.; Olson, A. J.; Spehner, J. C. Biopolymers 1996, 38, 305-320.

[45] Carr, T. W.; Baim, M. A.; Cohen, M. J.; Dam, R. J.; Hagen, D. F.; Hill, H. H.; Mason, E. A.; Siegel, M. W.; Spangler, G. E.; Wernlund, R. F. Plasma Chromatography; Plenum Press: New York, 1984.

[46] Wannier, G. H. Bell Syst. Tech. J. 1953, 32, 170-254. 


\section{Chapter 7}

\section{Interfacial Reactions of Ozone with Lipids and Proteins in a Model Lung Surfactant System}

\subsection{Abstract}

Oxidative stresses from irritants such as hydrogen peroxide and ozone $\left(\mathrm{O}_{3}\right)$ can cause dysfunction of the pulmonary surfactant (PS) in the human lung, resulting in chronic diseases of the respiratory tract. For identification of structural changes of major components of PS due to the heterogeneous reaction with $\mathrm{O}_{3}$, field induced droplet ionization (FIDI) mass spectrometry is utilized to probe the surfactant layer system. FIDI is a soft ionization method in which ions are extracted from the surface of micro liter volume droplets. We report the structurally specific oxidative changes of $\mathrm{SP}_{-} \mathrm{B}_{1-25}$ (a shortened version of human surfactant protein B) and 1-palmitoyl-2-oleoyl-sn-phosphatidylglycerol (POPG) due to reaction with $\mathrm{O}_{3}$ at the air-liquid interface. We also present studies of the interfacial oxidation of SP-B $\mathrm{B}_{1-25}$ in a non-ionizable 1-palmitoyl-2-oleoyl-sn-glycerol monolayer as a model lung surfactant system, where the competitive oxidation of the two components is observed. Our results indicate that the heterogeneous reaction at the interface is different from that in the bulk phase. For example, we observe the hydroxyhydroperoxide and the secondary ozonide as major products of the heterogeneous ozonolysis of POPG. These products are metastable and difficult to observe in the bulk-phase. In addition, compared to the nearly complete homogeneous oxidation of SP-B ${ }_{1-25}$, only a subset of the amino acids known to react with ozone is oxidized in the hydrophobic interfacial environment. Combining these experimental observations with the results of molecular dynamics simulations provides an improved understanding of the interfacial structure and chemistry of a model lung surfactant system when subject to oxidative stress. 


\subsection{Introduction}

The human lung is constantly exposed to airborne environmental insults. Long-term and immediate exposure of lungs to pathogens, air pollutants, and other irritants can be a major cause of acute and chronic injuries such as cardiopulmonary mortality and lung cancer $[1,2,3]$. Lung disease is the third leading cause of death in the United States and $\sim \$ 154$ billion is spent for direct and indirect lung disease-related health care every year [3]. Lung disease death rates are still increasing and more efforts to understand the chemical as well as the physical characteristics of lung system are required.

Pulmonary surfactant (PS) is a complex mixture of lipids and proteins [4] found in the lungs that reduces the surface tension of the alveolar sacs during the breath cycle. Phospholipids form oriented monolayers at the air-liquid interface. The principal phospholipid component of the layer, 1,2dipalmitoyl-sn-phosphatidylcholine (DPPC), can achieve a very low surface tension $(\sim 0 \mathrm{mN} / \mathrm{m})[5$, 6] while the higher fluidity of unsaturated phospholipids such as 1-palmitoyl-2-oleoyl-sn-phosphatidylglycerol (POPG) improves the adsorption and spreading properties of surfactant at the air-liquid interface [6]. Surfactant protein B (SP-B) enhances phospholipid adsorption and spreading from the sub-phase to the interface [7], and inherited deficiencies in SP-B are lethal at birth [8]. Despite its vital importance, little is known about the interactions of the protein with phospholipids to form final lipid-protein complexes in the PS [9] as well as the manner in which this complex environment modifies the interaction of reative oxygen species (ROS) with individual components.

A number of studies have reported the chemical changes of major components of PS under various oxidative stresses $[10,11,12]$, as well as changes in physical properties, which cause acute lung injury and respiratory failure $[1,13,14]$. For example, Uppu et al. used human red-blood cell membranes as a model lung system and demonstrated their oxidation via a bulk-phase $\mathrm{O}_{3}$ application [11]. The alteration of structure and physical properties of SP-B by ROS has been reported by Possmayer and co-workers $[12,14]$. Yet, understanding detailed mechanisms of chemical and physical changes in the complex PS system is still an active and challenging field of research. In particular, the oxidative change of major PS components by a heterogeneous air-liquid reaction with an external oxidative source (i.e. $\mathrm{O}_{3}$ ) has not been studied thoroughly at the molecular level. An increasing number of studies have focused on the heterogeneous chemistry of small molecules at the air-liquid interface, mainly using mass spectrometric [15] and spectroscopic [16] techniques, as well as theoretical methods [17]. Fewer studies have considered the air-liquid interfacial chemistry of biologically relevant systems. Exemplifying the latter, Colussi and co-workers recently reported heterogeneous reactions with $\mathrm{O}_{3}$ of ascorbic acid [18] and uric acid [19], which are components of the pulmonary epithelial lining fluid, using mass spectrometry.

Field induced droplet ionization mass spectrometry (FIDI-MS) comprises a soft ionization method to sample ions from the surface of microliter droplets $[15,20,21]$. It is ideally suited to monitor time 
dependent heterogeneous reactions at the air-liquid interface. A pulsed electric field stretches neutral droplets until they develop dual Taylor cones, emitting streams of positively and negatively charged submicron droplets in opposite directions. In practice, a quiescent hanging droplet is formed on the end of a capillary and then exposed to gas-phase reactants for a variable period of time, followed by FIDI-MS sampling of molecular species present in the interfacial layer (Figure 7.1).

In this study, we utilize FIDI-MS for probing air-liquid interfacial oxidation of POPG, representative of the major unsaturated anionic lipids in lung surfactant, and SP-B ${ }_{1-25}$ (FPIPLPYCWLCRALIKRIQAMIPKG) by $\mathrm{O}_{3}$. Synthetic $\alpha$-helical SP-B ${ }_{1-25}$ is reported to produce the same effect as the entire 79 amino acid SP-B [22, 23, 24]. Sampling droplets with an interfacial layer of POPG or $\mathrm{SP}-\mathrm{B}_{1-25}$ exposed to $\mathrm{O}_{3}$ gas over a range of reaction times reveals distinct air-liquid interfacial chemistry. In addition, we examine the heterogeneous reaction of a model PS system comprising SP-B $\mathrm{B}_{1-25}$ and 1-palmitoyl-2-oleoyl-sn-glycerol (POG) with $\mathrm{O}_{3}$. POG is non-ionizable lipid with properties at the air-liquid interface similar to POPG. It is employed to avoid undesired competition of ionization with SP-B ${ }_{1-25}$ at the surface of the droplet during the FIDI experiment. For comparison to the observed heterogeneous chemistry, we also report homogeneous reactions of SP-B ${ }_{1-25}$ with dissolved ozone. Structures of POPG, POG, and SP-B ${ }_{1-25}$ are shown in Figure 7.2. The observed interfacial reactions are correlated with positioning of SP-B $\mathrm{B}_{1-25}$ in a lipid monolayer as determined by means of molecular dynamic (MD) simulations.

\subsection{Methods}

\subsubsection{Chemicals and Reagents}

Sodium salts of POPG and POG were purchased from Avanti Polar Lipid (Alabaster, AL). SP$\mathrm{B}_{1-25}$ was purchased from Biomer Technology (Hayward, CA). All solvents were purchased from EMD Chemicals Inc. (Gibbstown, NJ).

\subsubsection{Online FIDI-MS Technique and Heterogeneous Oxidation by $\mathrm{O}_{3}$}

The FIDI-MS instrument used in this investigation were based on designs previously described by Grimm et al. [15]. A $\sim 2 \mathrm{~mm}$ o.d. droplet of analyte solution is suspended from the end of a 28-gauge stainless steel capillary (Small Parts Inc.). The droplet is located on center in the region between the plate electrode and the MS inlet; the plate and inlet were separated by $6 \mathrm{~mm}$. A high-voltage pulse $\left( \pm 7 \times 10^{5} \mathrm{~V} \mathrm{~m}^{-1}, 20 \mathrm{~ms}\right)$ is applied on the parallel plate electrode and sampling capillary to achieve FIDI. Ozonolysis reactions occur between 0 and $30 \mathrm{~s}$ after a quiescent droplet is achieved ( $\sim 1-2$ s). A pencil-style UV calibration lamp (model 6035, Oriel) generates $\sim 20 \mathrm{ppm}$ $\mathrm{O}_{3}$. $100 \mu \mathrm{M}$ POPG, $50 \mu \mathrm{M}$ SP-B ${ }_{1-25}$, or mixtures of $100 \mu \mathrm{M}$ POG and $50 \mu \mathrm{M}$ SP-B ${ }_{1-25}$ in 1:1 


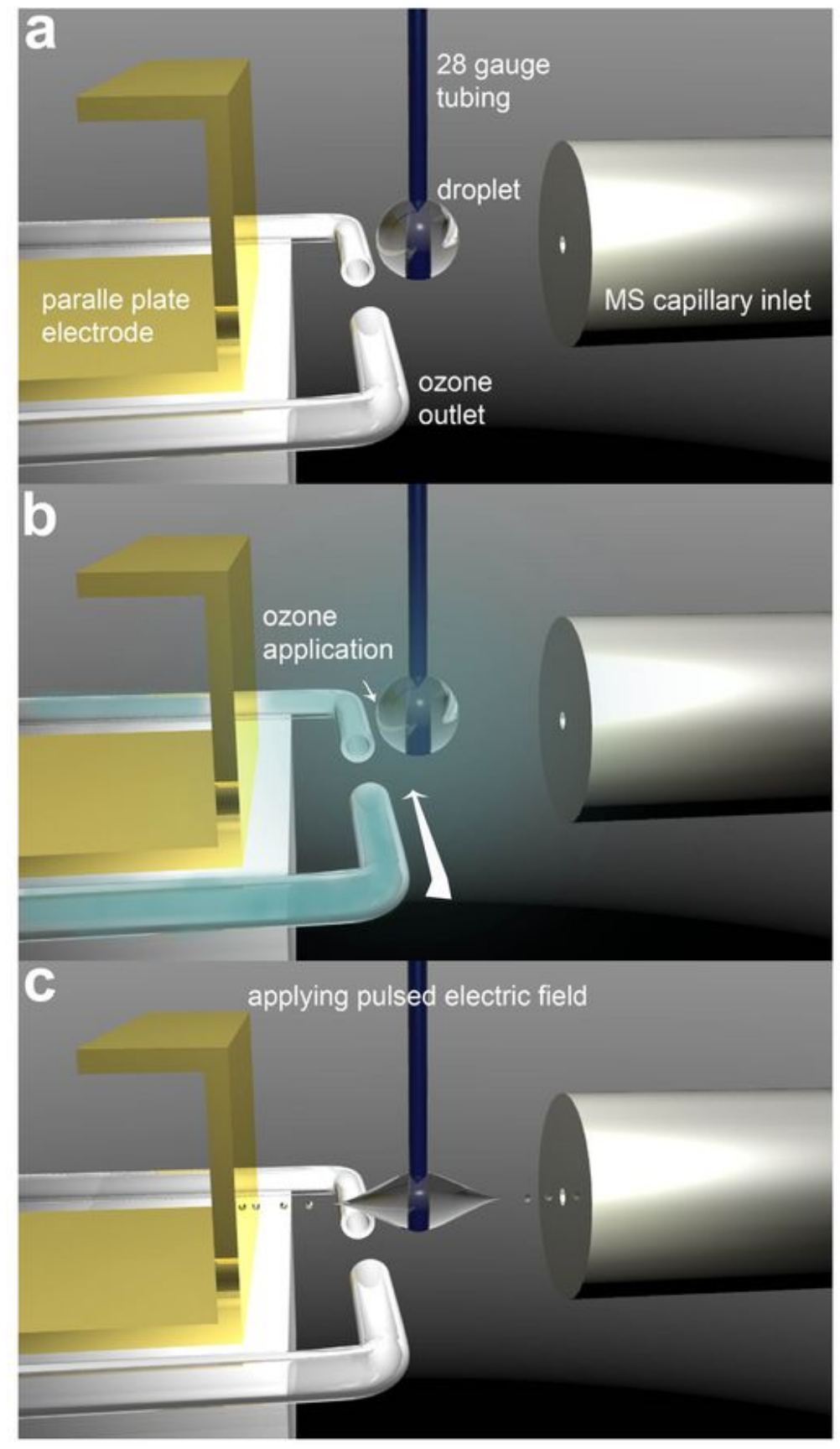

Figure 7.1: Illustration of FIDI-MS methodology for studies of interfacial reactions. (a) A quiescent hanging droplet of analyte-containing solution is formed on the end of a capillary. All electrical components remain at ground as the droplet is formed and reacts in a field-free environment. (b) The droplet is exposed to gas-phase reactants for a variable period of time to allow for heterogeneous reactions between gas-phase and solution-phase species. (c) After a reaciton period, a pulsed electric field stretches the neutral droplet until it emits stream of positively and negatively charged submicron droplets in opposite directions. Ionized reactants and products from heterogeneous reactions enter the capillary inlet of the mass analyzer. Either positive or negative ions can be sampled. 


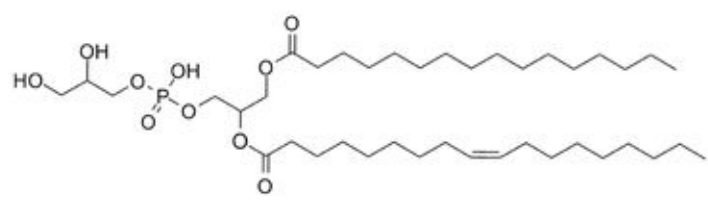

1-Palmitoyl-2-oleoyl-sn-phosphatidylglycerol

(POPG)

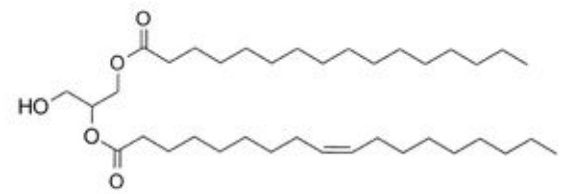

1-Palmitoyl-2-oleoyl-sn-glycerol

(POG)

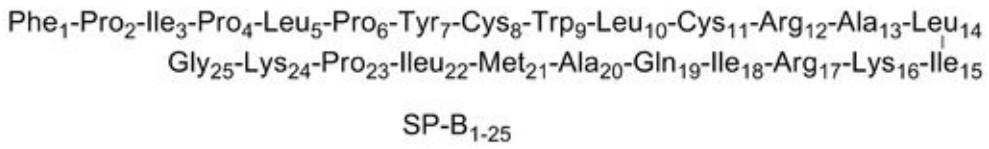

Figure 7.2: Structures of POPG, POG, and $\mathrm{SP}_{-} \mathrm{B}_{1-25}$ investigated in this study.

(by volume) water and methanol feed the droplet source. The FIDI-MS spectra reported in this study were obtained by averaging five to ten individually acquired spectra from separately prepared droplets. The $\mathrm{m} / \mathrm{z}$ of each ion was determined from the locations of the peak maxima identified in the mass spectra. The experimental details for the bulk-phase $\mathrm{O}_{3}$ application and the Fenton reaction of $\mathrm{SP}_{-} \mathrm{B}_{1-25}$ are described in Appendix $\mathrm{H}$.

\subsubsection{Molecular Dynamic Simulations}

The MD simulations were performed with the all-atom CHARMM PARAM27 [25] force field using the LAMMPS (large-scale atomic/molecular massively parallel simulator) code [26]. To describe the water, we used a flexible TIP3P potential, which needs additional Hooke's constants, $K$ of 900 $\mathrm{kcal} / \mathrm{mol} / \AA^{2}$ for $\mathrm{OH}$ bond and $K$ of $110 \mathrm{kcal} / \mathrm{mol} / \mathrm{rad}^{2}$ for $\mathrm{HOH}$ angle to the 3 -site-rigid TIP3P model [25]. The initial conformation of $\mathrm{SP}_{-} \mathrm{B}_{1-25}$ was taken from the Protein Data Bank structure (1DFW). The particle-particle particle-mesh (PPPM) method [27] was employed to compute the electrostatic using an accuracy criterion of $10^{-5}$.

The initial structures for the lipid monolayer-water systems were prepared with 48 hexagonallypacked lipids on the $3168,3264,3744$, and 4464 water molecules for the $55,60,65$, and $70 \AA^{2} /$ lipid surface densities, respectively. A pure-repulsive wall potential, $E=\epsilon\left[2 / 15(\sigma / r)^{9}-(\sigma / r)^{3}\right]$, where $\epsilon=0.1521 \mathrm{kcal} / \mathrm{mol}$ and $\sigma=3.1538 \AA$ with cut-off distance of $2.7071 \AA$, was applied at $z=0$ to 
a

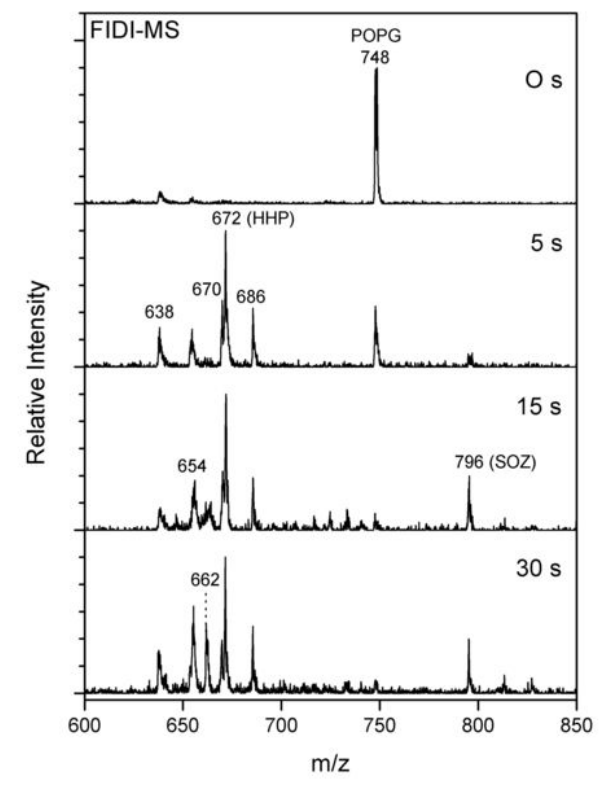

b
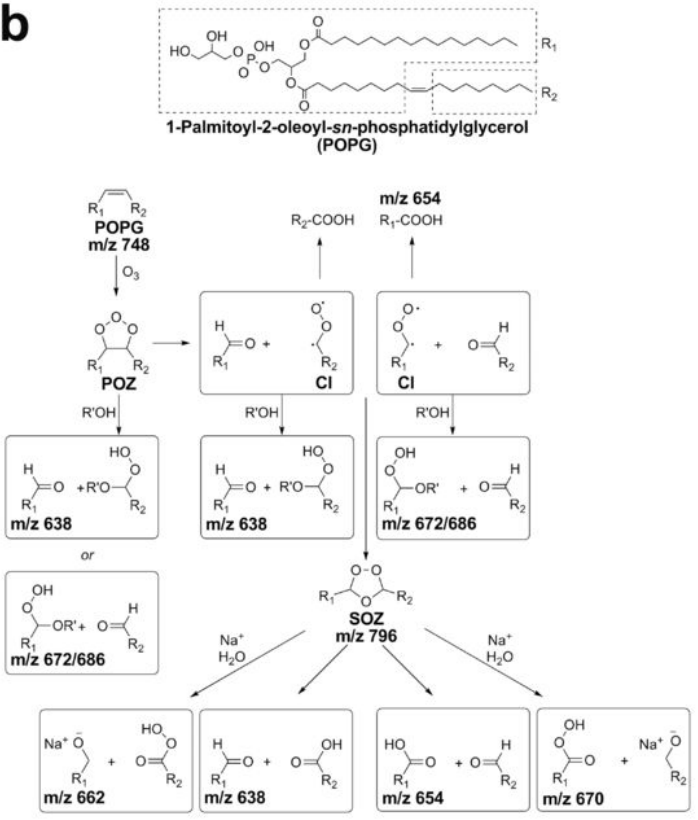

Figure 7.3: (a) Heterogeneous reaction of POPG with $\mathrm{O}_{3}$ as a function of time. In the absence of ozone, the negative ion FIDI-MS spectrum of POPG is dominated by the singly deprotonated POPG peak at $\mathrm{m} / \mathrm{z} 748$. POPG is depleted after $15 \mathrm{~s}$ of the exposure and oxidation products are dominated by deprotonated hydroxyhydroperoxide (HHP) at $\mathrm{m} / \mathrm{z} 672$. The aldehyde, carboxylic acid, and methoxyhydroperoxide products are observed at $\mathrm{m} / \mathrm{z} 638, \mathrm{~m} / \mathrm{z} 654$, and $\mathrm{m} / \mathrm{z} 686$, respectively. The secondary ozonide (SOZ) and sodiated alcohol products show up in the spectra at $\mathrm{m} / \mathrm{z} 796$ and $\mathrm{m} / \mathrm{z} 662$, respectively. (b) Summary of heterogeneous oxidation of POPG with $\mathrm{O}_{3}$ at the air-liquid interface. $\mathrm{R}^{\prime}$ is $\mathrm{H}$ for water and $\mathrm{CH}_{3}$ for methanol.

prevent the water from diffusing in the negative $z$-direction. The dimensions of the simulation cells used were $(55.21 \AA \times 47.82 \AA \times 200.0 \AA)$ for the $55 \AA^{2} /$ lipid, $(57.67 \AA \times 49.94 \AA \times 200.0 \AA)$ for the $60 \AA^{2} /$ lipid, $(60.02 \AA \times 51.98 \AA \times 200.0 \AA)$, for the $65 \AA^{2} /$ lipid, and $(62.28 \AA \times 53.94 \AA \times 200.0$ $\AA$ ) for the $70 \AA^{2} /$ lipid surface densities. The systems were equilibrated for $0.5 \mathrm{~ns}$ using $300 \mathrm{~K}$ NVT MD simulations by applying Nosé-Hoover thermostat with a temperature damping relaxation time of $0.1 \mathrm{ps}$. Then, $2.0 \mathrm{~ns}$ NVT MD simulations were performed, and these trajectories are employed for the analysis of the atomic profiles.

The initial structure for the SP-B $\mathrm{B}_{1-25}$ in the POG or POPG monolayer was constructed using the final structure after the simulation of the lipid monolayer-water system with the surface density of $60 \AA^{2}$. After removing six neighboring lipids, the SP-B ${ }_{1-25}$ is inserted into the resultant cavity with an $\alpha$-helical axis orientation angle of $34^{\circ}$ to the interfacial plane. Then the dimensions of the simulation cells were slightly adjusted to $(57.88 \AA \times 50.12 \AA \times 200.0 \AA)$. Similar to the lipid monolayer simulations, 0.5 ns equilibration followed by 2.0 ns NVT MD simulation was performed at $300 \mathrm{~K}$. To analyze the trajectories, we averaged the population over the last $0.5 \mathrm{~ns}$ of the $2.0 \mathrm{~ns}$ trajectories. 


\subsection{Results and Discussion}

\subsubsection{Interfacial Reaction of POPG with $\mathrm{O}_{3}$}

The cis-double bond of an unsaturated phospholipid reacts with $\mathrm{O}_{3}$ yielding aldehyde and carboxylic acid products directly from primary ozonide (POZ) or through energetic Crigee intermediates (CI), while saturated phospholipids such as DPPC remain intact. In this study we have investigated the heterogeneous reaction of $\mathrm{O}_{3}$ with POPG as a representative unsaturated phospholipid in PS system. The negative ion FIDI-MS spectra for ozonolysis of POPG in a water/methanol (1:1 by volume) droplet are shown in Figure 7.3a along with the proposed reaction mechanisms (Figure 7.3b). Singly deprotonated POPG, observed at $\mathrm{m} / \mathrm{z} 748$, is seen as a dominant species in the FIDI-MS spectrum before $\mathrm{O}_{3}$ application (Figure 7.3a). Products resulting from ozonolysis of POPG appear at least as early as $5 \mathrm{~s}$ after exposing the droplet to $\mathrm{O}_{3}$. The relative abundance of the reactant POPG decreases dramatically after $15 \mathrm{~s}$ of exposure, and then the FIDI-MS spectrum is dominated by ozonolysis products after $30 \mathrm{~s}$. The time for consuming $90-99 \%$ of POPG to form the primary ozonide (POZ) at the air-liquid interface is calculated to be $\sim 10-20 \mathrm{~s}$ (see Appendix G). This agrees well with the experimental observation of this study.

It is noteworthy that hydroxyhydroperoxide (HHP), methoxyhydroperoxide (MHP), and the $\mathrm{SOZ}$, which are known to be metastable species in the bulk-phase, are observed as major products of POPG ozonolysis in the FIDI-MS spectra (Figure 7.3a) [28]. In order to yield HHP, a Criegee intermediate (CI) or a POZ is required to react with a water molecule $[28,29]$. Rapid decomposition of HHP through proton transfers from water molecules yields ROS [29], which makes it difficult to observe HHP directly in the bulk-phase. The water density at the air-liquid interfacial region is significantly lower than in the bulk-phase [30]. In addition, water molecules in a lipid monolayer are observed to be localized within the lipid head group region due to the strong interactions with polar head groups [31]. These conditions allow HHP to be abundant in the lipid monolayer at the air-liquid interface, which is a characteristic of the heterogeneous reaction of POPG compared to the homogeneous reaction [29]. The observed MHP originates from the reaction of a CI or POZ with a methanol molecule in the droplet.

A significant abundance of SOZ is observed in the FIDI-MS spectra after exposing the droplet to $\mathrm{O}_{3}$ for $15 \mathrm{~s}$. The structure of SOZ (m/z 796) is confirmed by low energy collision induced dissociation (CID), which yields the aldehyde (m/z 638) and carboxylic acid (m/z 654) fragments. The peak corresponding to SOZ continues to build up in the spectrum as the POPG lipid is depleted. We infer that the observed $\mathrm{SOZ}$ is not formed by direct rearrangement of $\mathrm{POZ}$ but rather by recombination of the CI with aldehydes (Figure 7.3b) $[28,32]$. In the bulk-phase, however, faster reaction with water molecules prevents the CI from reacting with aldehyde to form SOZ [33]. A significant amount of the sodiated alcohol product ( $\mathrm{m} / \mathrm{z} 662)$ is observed after exposing the droplet to $\mathrm{O}_{3}$ for $30 \mathrm{~s}$. This 
a

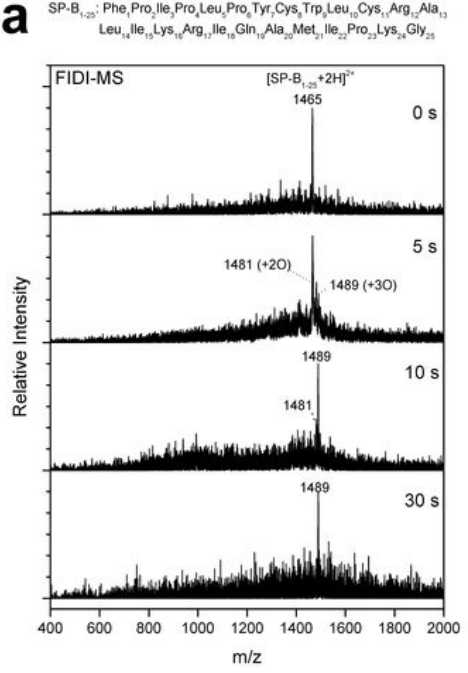

b

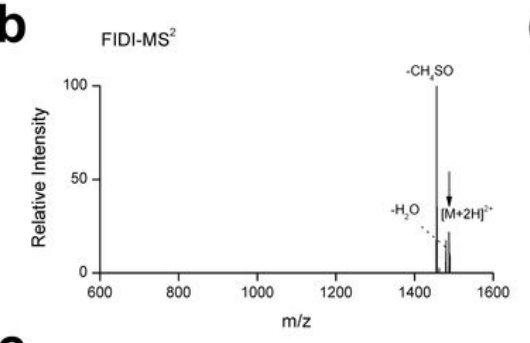

C

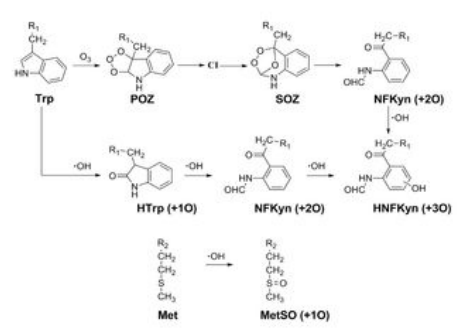

d

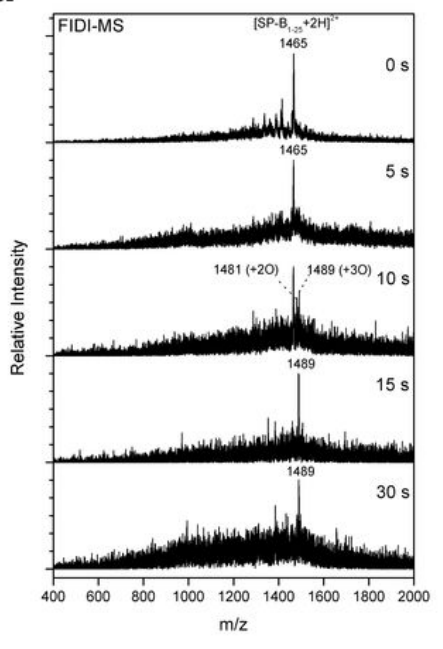

Figure 7.4: (a) Air-liquid interfacial oxidation of $\mathrm{SP}_{-} \mathrm{B}_{1-25}$ by $\mathrm{O}_{3}$ as a function of time. In the absence of ozone, the positive ion FIDI-MS spectrum of SP-B ${ }_{1-25}$ is dominated by the doubly protonated $\mathrm{SP}_{-} \mathrm{B}_{1-25}$ peak at $\mathrm{m} / \mathrm{z} 1465$. The products at $\mathrm{m} / \mathrm{z} 1481$ and $\mathrm{m} / \mathrm{z} 1489$ correspond to doubly protonated $\mathrm{SP}-\mathrm{B}_{1-25}$ with two oxygen atoms and with three oxygen atoms, respectively, appear after the droplet is exposed to $\mathrm{O}_{3}$ for $5 \mathrm{~s}$. The triply oxygenated product at $\mathrm{m} / \mathrm{z} 1489$ dominates the FIDI-MS spectrum after exposing the droplet to $\mathrm{O}_{3}$ for $10 \mathrm{~s}$. No further oxidation of the peptide is observed up to $30 \mathrm{~s}$ of exposure. (b) FIDI-MS ${ }^{2}$ of doubly charged triply oxygenated $\mathrm{SP}-\mathrm{B}_{1-25}$ product from heterogeneous oxidation yields an exclusive fragment at $\mathrm{m} / \mathrm{z} 1457$ resulting from the elimination of hydrosulfinylmethane $\left(\mathrm{CH}_{4} \mathrm{SO}\right)$. (c) The oxidation mechanisms of Trp by ozonolysis and hydrolysis with hydrolysis mechanism of Met in peptide. (d) Air-liquid interfacial oxidation of $\mathrm{SP}-\mathrm{B}_{1-25}$ by $\mathrm{O}_{3}$ in the POG monolayer as a function of time. Doubly protonated SP-B $B_{1-25}$ products with two oxygen atoms and with three oxygen atoms appear after the droplet is exposed to $\mathrm{O}_{3}$ for $10 \mathrm{~s}$. The triply oxygenated product dominates the FIDI-MS spectrum after exposing the droplet to $\mathrm{O}_{3}$ for $15 \mathrm{~s}$ and no further oxidation of the peptide is observed up to $30 \mathrm{~s}$ of exposure.

product is due to the dissociation of SOZ followed by the association with sodium cation. This suggests that after SOZ is produced under an anhydrous environment, the newly formed hydrophilic molecule interacts with sodium cation in the liquid-phase to yield the sodiated alcohol product. These SOZ and sodiated alcohol products are characteristic of specific air-liquid interface chemistry during POPG ozonolysis.

\subsubsection{Interfacial Oxidation of SP-B $1-25$}

The positive ion FIDI-MS spectra for the oxidation of $\mathrm{SP}-\mathrm{B}_{1-25}$ by $\mathrm{O}_{3}$ are shown in Figure 7.4a. The doubly protonated SP-B $\mathrm{B}_{1-25}$ is observed as the dominant species in the FIDI-MS spectrum before $\mathrm{O}_{3}$ application. Products resulting from the oxidation of $\mathrm{SP}-\mathrm{B}_{1-25}$ by $\mathrm{O}_{3}$ appear after the droplet is exposed to $\mathrm{O}_{3}$ for $5 \mathrm{~s}$. The products at $\mathrm{m} / \mathrm{z} 1481$ and $\mathrm{m} / \mathrm{z} 1489$ correspond to doubly protonated $\mathrm{SP}-\mathrm{B}_{1-25}$ with two oxygen atoms and with three oxygen atoms, respectively. The FIDIMS spectrum of the droplet with $\mathrm{SP}_{-} \mathrm{B}_{1-25}$ is dominated by the triply oxygenated product at $\mathrm{m} / \mathrm{z}$ 
1489 after exposing the droplet to $\mathrm{O}_{3}$ for $10 \mathrm{~s}$. No further oxidation of the peptide is observed up to $30 \mathrm{~s}$ of exposure. The FIDI-MS spectra show that the doubly oxygenated product immediately undergoes further oxidation to form the stable product with three oxygen atoms.

The FIDI collision induced dissociation (CID) spectrum (FIDI-MS ${ }^{2}$ ) of the product at m/z 1489 is shown in Figure 7.4b. The CID of triply oxygenated SP-B ${ }_{1-25}$ from heterogeneous oxidation yields an exclusive fragment at $\mathrm{m} / \mathrm{z} 1457$ resulting from the elimination of hydrosulfinylmethane $\left(\mathrm{CH}_{4} \mathrm{SO}\right)$, which is the characteristic CID fragment of methionine sulfoxide (MetSO) [34]. This indicates that the oxidation of the methionine residue $\left(\mathrm{Met}_{21}\right)$ in SP-B ${ }_{1-25}$ results from the heterogeneous ozonolysis. The other two oxygen atoms are added to the tryptophan residue ( $\left.\operatorname{Trp}_{9}\right)$ forming $\mathrm{N}$ formylkynrenine (NFKyn) (see Appendix H).

In order to investigate the difference between the interfacial and bulk-phase reactions of $\mathrm{O}_{3}$ with SP-B ${ }_{1-25}, \mathrm{O}_{3}$ was bubbled into a solution containing SP-B ${ }_{1-25}$. Oxidation using the Fenton reaction is also performed to compare the bulk-phase ozonolysis to bulk-phase oxidation by $\mathrm{OH}$ radical (see Appendix $\mathrm{H}$ for detailed analysis). The Fenton reaction yields a series of oxidized $\mathrm{SP}_{-} \mathrm{B}_{1-25}$ products with up to 10 oxygen atoms while the bulk-phase $\mathrm{O}_{3}$ reaction yields dominating products of 3 - and 9 -oxygenated SP-B ${ }_{1-25}$. For the comparison to heterogeneous ozonolysis of SP-B ${ }_{1-25}$, the products with three additional oxygen atoms from both reactions are analyzed. Bulk-phase $\mathrm{O}_{3}$ application yields two triply oxygenated SP- $\mathrm{B}_{1-25}$ products, one with NFKyn $(+2 \mathrm{O})$ and MetSO $(+1 \mathrm{O})$, and the other with hydroxy-N-formylkynrenine $(\mathrm{HNFKyn},+3 \mathrm{O})$. The Fenton reaction yields only the product with HNFKyn.

Ozone has limited solubility in water. For a gas-phase concentration of $20 \mathrm{ppm} \mathrm{O}_{3}$, the equilibrium concentration of $\mathrm{O}_{3}$ dissolved in aqueous solution is calculated as $22.6 \mathrm{nM}$ by Henry's law [35]. In addition, $\mathrm{O}_{3}$ is unstable in water, and rapidly forms secondary oxidants [36]. The major secondary oxidant formed by $\mathrm{O}_{3}$ in water is $\mathrm{OH}$ radical [37]. This implies that two major $\mathrm{O}_{3}$ oxidation pathways, ozonolysis and hydroxylation, can be observed at the air-liquid interface. The triply oxygenated SP-B $\mathrm{B}_{1-25}$ is formed concomitantly with the formation of the doubly oxygenated SP- $\mathrm{B}_{1-25}$ after exposing the droplet to $\mathrm{O}_{3}$ for $5-10 \mathrm{~s}$ (Figure 7.4a). The oxidation mechanisms of Trp and Met in peptide are shown in Figure 7.4c. The NFKyn can be formed via direct ozonolysis of Trp [38] or hydrolysis of hydroxytryptophan (HTrp) [12]. However, the formation of MetSO from Met occurs primarily by secondary oxidants [39]. The NFKyn of the heterogeneous reaction results from the direct ozonolysis of Trp 9 , which is located at the hydrophobic N-terminal side of SP-B $\mathrm{B}_{1-25}$. $\mathrm{Met}_{21}$, which likely forms MetSO, is located at the hydrophilic C-terminal site. Intact $\mathrm{Cys}_{8}$ and $\mathrm{Cys}_{11}$ support this observed $\mathrm{O}_{3}$ oxidation of SP-B $\mathrm{B}_{1-25}$. The rate constant for reaction of Cys with ozone is three orders of magnitude larger than that of Trp and Met [40]. However, the oxidation of Cys to yield sulfonic acid occurs primarily by reactions with secondary oxidants [41], which are not present in significant concentration in the vicinity of the hydrophobic segment of the peptide where 
$\mathrm{Cys}_{8}$ and $\mathrm{Cys}_{11}$ are located. The formation of HNFKyn requires at least one secondary oxidation step (Figure 7.4c). After NFKyn is formed by either direct ozonolysis or reaction with secondary oxidants, the Met and NFKyn undergo competitive oxidation to yield MetSO $\left(k=1.4 \times 10^{-11} \mathrm{~cm}^{3}\right.$ molecule $\left.{ }^{-1} \mathrm{~s}^{-1}\right)$ and HNFKyn $\left(k=1.3 \times 10^{-11} \mathrm{~cm}^{3}\right.$ molecule $\left.{ }^{-1} \mathrm{~s}^{-1}\right)$ by ROS in the bulk-phase [42]. The heterogeneous ozonolysis of $\operatorname{Trp}_{9}$ induces a change in peptide orientation at the interface. This results in $\mathrm{Cys}_{8}$ and $\mathrm{Cys}_{11}$ being exposed to ROS for oxidation in the bulk phase, which yields total 9-oxygenated SP-B ${ }_{1-25}$.

\subsubsection{Oxidation of $\mathrm{SP}-\mathrm{B}_{1-25}$ in POG Monolayer by $\mathrm{O}_{3}$}

The interfacial reaction of $\mathrm{SP}_{-} \mathrm{B}_{1-25}$ with ozone was also examined in a monolayer of the nonionizable lipid POG. Almost identical FIDI-MS spectra are observed compared to the spectra obtained for ozonolysis of $\mathrm{SP}_{-} \mathrm{B}_{1-25}$ without POG except for an $\sim 5 \mathrm{~s}$ time delay for initiation of the reaction (Figure 7.4d). The FIDI-MS spectrum of the $\mathrm{SP}_{-1-25} / \mathrm{POG}$ droplet is dominated by the triply oxygenated product after $15 \mathrm{~s}$ of exposure. No further oxidation of the peptide is observed up to $30 \mathrm{~s}$ exposure.

The observed time delay of the reaction provides a critical clue regarding the location of SP$\mathrm{B}_{1-25}$ in the monolayer. From the FIDI-MS spectra, it takes $\sim 10-20 \mathrm{~s}$ for POPG at the air-liquid interface to be consumed by $\mathrm{O}_{3}$ (Figure 7.3a). Both POG and POPG possess a palmitic acid chain and an oleic acid chain, the latter of which reacts with $\mathrm{O}_{3}$. Under the assumption that the reactivity of POG is similar to that of POPG, the initiation of the $\mathrm{SP}^{-\mathrm{B}_{1-25}}$ ozonolysis is expected after the droplet is exposed to $\mathrm{O}_{3}$ for $10-20 \mathrm{~s}$, if the peptide is completely shielded by lipid acyl chains. However, the observed short time delay of the initiation of SP- $\mathrm{B}_{1-25}$ ozonolysis suggests that the peptide competes directly with POG for reaction with $\mathrm{O}_{3}$. This is consistent with a picture in which the peptide is colocated at the air-liquid interface with POG at the surface of the droplet.

\subsubsection{Interactions of SP-B ${ }_{1-25}$ in a Lipid Monolayer}

We carried out MD simulations for the POPG monolayer and POG monolayer in a water box for $2.0 \mathrm{~ns}$ with four different surface densities $\left(55,60,65\right.$, and $70 \AA^{2} /$ lipid), which are reported as a proper density range for pulmonary surfactant function from previous theoretical studies [43, 44, 45]. Figure 7.5 shows the atomic density profiles of oxygen atoms of water molecules, saturated carbon atoms, and unsaturated carbon atoms of lipid acyl chains along $\pm \Delta z$, which is $z$-direction relative to the averaged phosphorous atom of POPG or hydroxyl hydrogen of POG. The POPG monolayer and POG monolayer exhibit almost identical atomic density profiles. Slightly stronger interaction between POPG and water is observed from their larger area of overlapping density ( $\sim 1.6$ times). This is due to the strong ion-dipole interactions between POPG phosphate group 


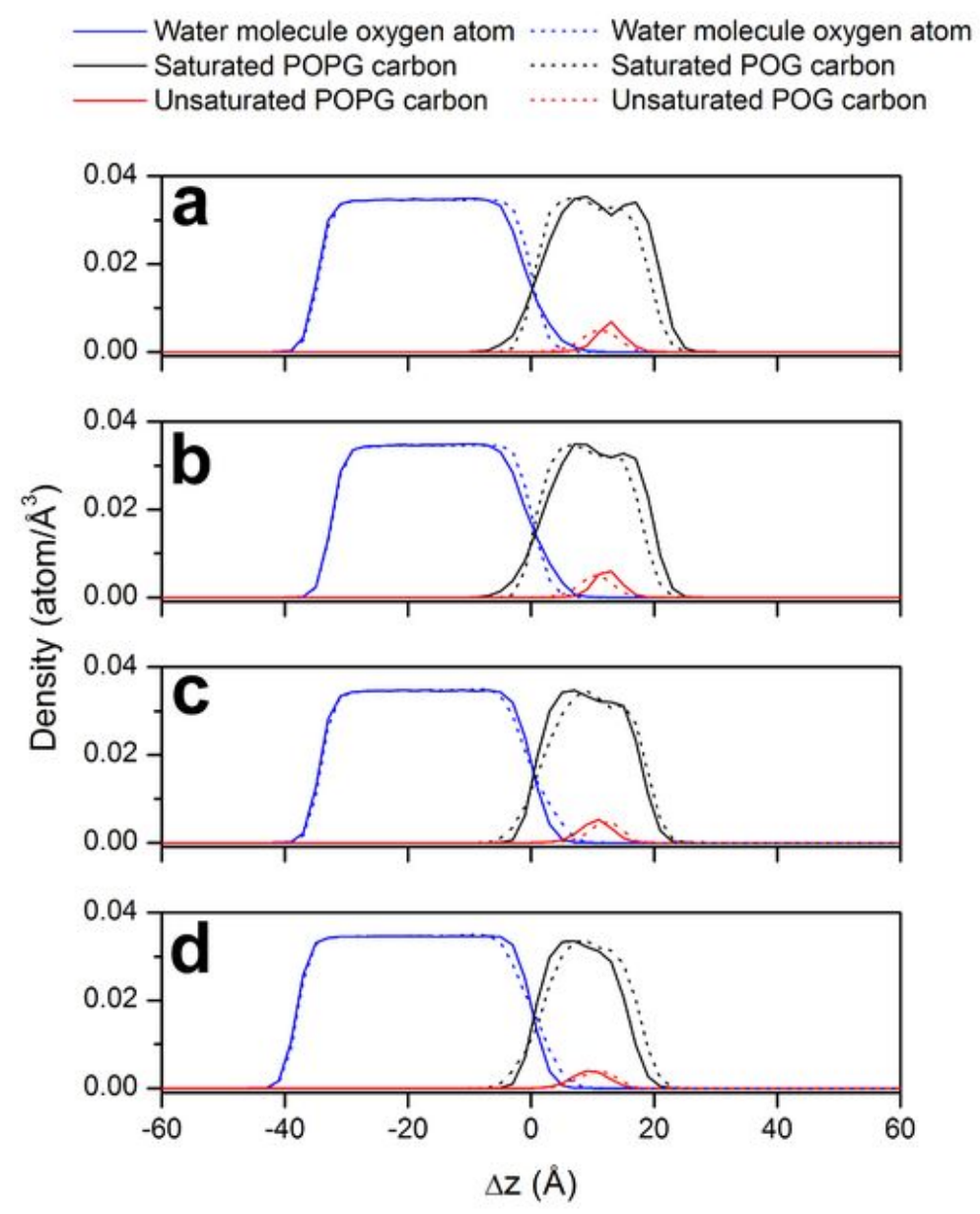

Figure 7.5: Atomic density profiles of POPG monolayer systems (solid lines) and POG monolayer systems (dotted lines) as a function of $\Delta z$, where the air/liquid interface is 0 , and proceeding left to right from the water to the lipid layer. The lipid surface densities are (a) $55 \AA^{2} /$ lipid, (b) 60 $\AA^{2} /$ lipid, (c) $65 \AA^{2}$ /lipid, and (d) $70 \AA^{2}$ /lipid. Blue lines denote the density profiles of oxygen atoms of water molecules, black lines denote that of saturated carbons of lipid acyl chains, and red lines denote that of unsaturated carbons of lipid acyl chains. 

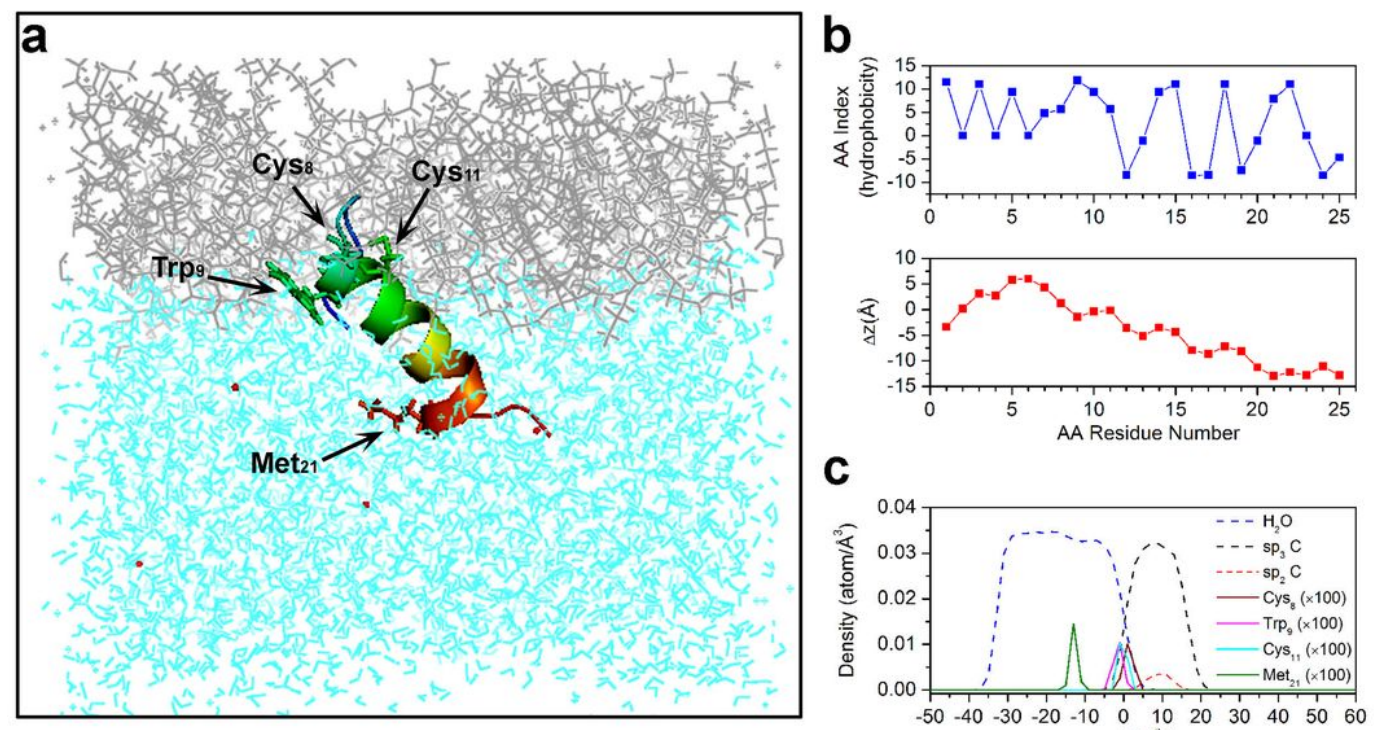

C

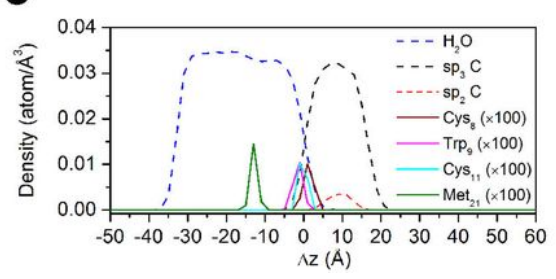

Figure 7.6: (a) Final snapshot after 2.0 ns of MD simulation of $\mathrm{SP}_{-1-25}$ in a POG monolayer at 60 $\AA^{2} /$ lipid. The peptide is shown in rainbow color (C-terminal: red, N-terminal: blue). Lipids, water molecules, and chloride are shown in purple, cyan, and red, respectively. (b) AA hydrophobicities [46] (top) and $\Delta z$ of $\mathrm{C}_{\alpha}$ of each residue averaged during the last 0.5 ns of a 2.0 ns duration MD simulation (bottom) are plotted as a function of amino acid residue number. The air/water interface is located near $\Delta z=0$. (c) Atomic density profiles of SP-B $\mathrm{B}_{1-25}$ in POG monolayer at $60 \AA^{2} /$ lipid as a function of $\Delta z$ during the last $0.5 \mathrm{~ns}$ of the $2.0 \mathrm{~ns} \mathrm{MD}$ simulation. Blue dash line denotes the density profiles of oxygen atoms of water molecules. Black and red dash lines denote those of separately summed saturated and unsaturated carbons of lipid acyl chains, respectively. Wine, magenta, cyan, and olive solid lines denote the 100 times scaled density profiles of the $\mathrm{C}_{\alpha}$ carbon of $\mathrm{Cys}_{8}, \mathrm{Trp}_{9}, \mathrm{Cys}_{11}, \mathrm{Met}_{21}$ residues, respectively.

and water molecules, which is absent from the POG monolayer. The water density at the double bond of POPG $(5-20 \AA)$ is $\sim 0.0005$ atom $/ \AA^{3}$, which is $\sim 70$ times less dense than in the bulk-phase $\left(\sim 0.035\right.$ atom $\left./ \AA^{3}\right)$. The low water concentrations around the double bond explains the experimental observation of the intermediates and metastable products from heterogeneous ozonolysis, including SOZ, HHP, and MHP (Figure 7.3a), which are difficult to observe in water-rich environments [28, 29, 33].

We performed 2.0 ns duration MD simulations of the POG/SP-B ${ }_{1-25} /$ water monolayer with 60 $\AA^{2} /$ lipid surface density as a representative case. The final snapshot in Figure 7.6a shows that the $\mathrm{SP}_{-\mathrm{B}_{1-25}}$ is located at the air-liquid interface. The hydrophobicity index of each amino acid (AA) residue in the peptide is shown in Figure 7.6b (top) [46]. Relatively strong hydrophobicity is found for the N-terminal side of the peptide with Leu, Ile, and Pro residues. In contrast, hydrophilicity is expected from C-terminal side due to Arg, Lys, and Gln residues. The MD simulated $\Delta z$ of $\mathrm{C}_{\alpha}$ of each residue exhibits a good correlation with the hydrophobicity index. The hydrophobic N-terminal side of the peptide is located above the air-liquid interface, while the hydrophilic C-terminal side is 
a

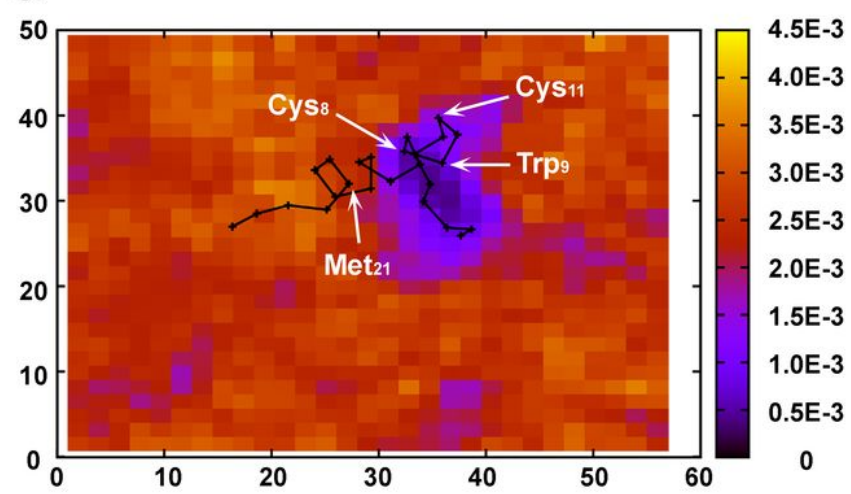

\section{b}

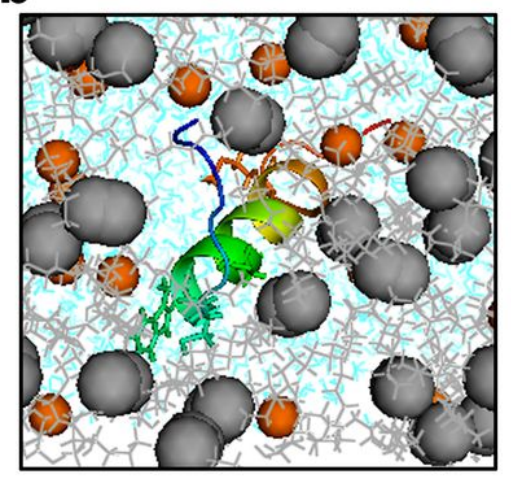

Figure 7.7: (a) The $x y$-projected density profiles of saturated carbon atoms of lipid acyl chains from MD simulations is shown with colors and the averaged positions of $\mathrm{C}_{\alpha}$ carbons of SP-B $\mathrm{B}_{1-25}$ in the POG monolayer is shown with a black line (each residue is shown with cross). (b) Top view of final snapshot after $2.0 \mathrm{~ns}$ of MD simulation of $\mathrm{SP}_{-\mathrm{B}_{1-25}}$ in a POG monolayer at $60 \AA^{2} /$ lipid. The peptide is shown in rainbow color (C-terminal: red, N-terminal: blue). Lipids and water molecules are shown in gray and cyan, respectively. Black spheres denote unsaturated carbon atoms of lipid acyl chains, and orange spheres denote hydroxyl oxygen atoms.

located under the interface (Figure 7.6b bottom). Figure 7.6c shows the atomic density profiles of oxygen atoms of water molecules as well as saturated and unsaturated carbon atoms of POG acyl chains along $\Delta z$. The 100 times scaled atomic density profiles of the $\mathrm{C}_{\alpha}$ carbon of $\mathrm{Cys}_{8}, \operatorname{Trp}_{9}, \mathrm{Cys}_{11}$, and Met $_{21}$ residues are also shown in Figure 7.6c. The density profiles show a good agreement with the hydrophobicity index. In practice, a low water density is found around $\operatorname{Trp}_{9}$, which leads to the formation of NFKyn via direct ozonolysis. The water density around Met $_{21}$ is observed to be sufficiently high to expect ROS formation and subsequent reaction to yield MetSO. In contrast, the low water density near $\mathrm{Cys}_{8}$ and $\mathrm{Cys}_{11}$ inhibits their oxidation by ROS.

Based on the competitive reactivity of POG and SP- $\mathrm{B}_{1-25}$ with $\mathrm{O}_{3}$ we suggest above that they are colocated at the interface. The MD simulations of SP-B $\mathrm{B}_{1-25}$ in a lipid monolayer support our interpretation. Trp 9 and $\mathrm{Met}_{21}$ of $\mathrm{SP}^{-\mathrm{B}_{1-25}}$ lie below (in the $z$-direction) the location of the lipid double bonds (Figure 7.6c). Figure 7.7a shows the $x y$-projected density profiles of saturated carbon atoms of lipid acyl chains with the averaged positions of $\mathrm{C}_{\alpha}$ of $\mathrm{AA}$ residues of $\mathrm{SP}-\mathrm{B}_{1-25}$. It is noteworthy that lipid acyl chains do not shelter the peptide at the air-liquid interface. The top view of the MD simulation final snapshot in Figure 7.7b illustrates that SP-B ${ }_{1-25}$ is not shielded by unsaturated carbons (black spheres) of lipids. The strong amphiphilic characteristic and the large surface area of SP-B ${ }_{1-25}$ cause the peptide to position itself at the air-liquid interface where it displaces lipids. As a result, $\mathrm{SP}-\mathrm{B}_{1-25}$ forms an island in a lipid monolayer that causes the hydrophobic portion of the peptide to be exposed to $\mathrm{O}_{3}$ despite its location below the position of lipid double bonds. We also simulated SP-B $\mathrm{B}_{1-25}$ in the POPG monolayer. The peptide penetrates 
deeper into the POPG monolayer due to the strong electrostatic interaction between the cationic AA residues $\left(\operatorname{Arg}_{12}, \operatorname{Arg}_{17}\right.$, and $\left.\mathrm{Lys}_{16}\right)$ and the anionic phosphate group of POPG (Figures 7.8a and 7.8b), which agrees with previous simulations in anionic lipid monolayers [43, 47]. SP-B $1-25$ forms an island in the POPG monolayer and the anhydrous environments in the lipid monolayer may lead SP-B ${ }_{1-25}$ to be more susceptible to direct ozonolysis than to modification by ROS (Figures $7.8 \mathrm{c}$ and $7.9)$.

\subsection{Conclusions}

Summarizing, to understand the unique chemistry at a model lung surfactant/air interface under $\mathrm{O}_{3}$ exposure, we utilized the FIDI-MS technique to analyze chemical reactions at this interface. In the FIDI-MS spectra, oxidized products distinct from those formed in the bulk-phase were observed from SP-B ${ }_{1-25}$ alone and imbedded in the POG monolayer. We also carried out MD simulations that provide additional insights into the interactions between lipids, $\mathrm{SP}-\mathrm{B}_{1-25}$, and water molecules in the interfacial region. In these simulations the location of $\mathrm{SP}-\mathrm{B}_{1-25}$ relative to the lipids provides a rationalization for the experimental observation that the peptides compete with the lipids for reaction with $\mathrm{O}_{3}$.

The oxidation of PS causes surface dysfunction in adsorption, respreading, and reduction of surface tension $[14,48]$. Once the $\mathrm{O}_{3}$ traverses the air-liquid interface, it decays rapidly concomitant with the formation of ROS in regions with high water densities [36]. However, due to the high reactivity with PS at the interface, it has been thought that little or none of the $\mathrm{O}_{3}$ can penetrate the PS monolayer to attack the epithelium cells below [49]. Instead of direct attack by $\mathrm{O}_{3}$ and its ROS derivates, secondary oxidized products of PS, such as HHP, have been expected to yield cellular damage [49]. We have found that more than $60 \%$ of the heterogeneous oxidation products of POPG by $\mathrm{O}_{3}$ are peroxides. These products, which are more water soluble than others, eventually dissolve into the water droplet. Then, rapid decomposition of products yields reactive oxygen species (ROS) [29] which causes cellular damage below the monolayer.

Possible protection of SP-B from homogeneous oxidation by PS lipids has been suggested in an earlier study [12]. However, we have shown that SP-B ${ }_{1-25}$ is oxidized directly by heterogeneous reaction with $\mathrm{O}_{3}$ since it is located at the air-liquid interface with significant exposure to $\mathrm{O}_{3}$. The homogeneous oxidation of SP-B by ROS is known to reduce its surface activity and function [12, 14]. We have observed fast formation of NFKyn from the direct ozonolysis of $\operatorname{Trp}_{9}$ at the hydrophobic N-terminal side of SP-B $\mathrm{B}_{1-25}$. The oxidized residue reduces the hydrophobicity of the N-terminal side of the peptide. This induces a change in peptide orientation in the monolayer that results in NFKyn, $\mathrm{Cys}_{8}$, and $\mathrm{Cys}_{11}$ being exposed to ROS for further oxidation. As a result, further oxidation of SP$\mathrm{B}_{1-25}$ by a heterogeneous reaction with $\mathrm{O}_{3}$ produces effects similar to those seen in homogeneous 

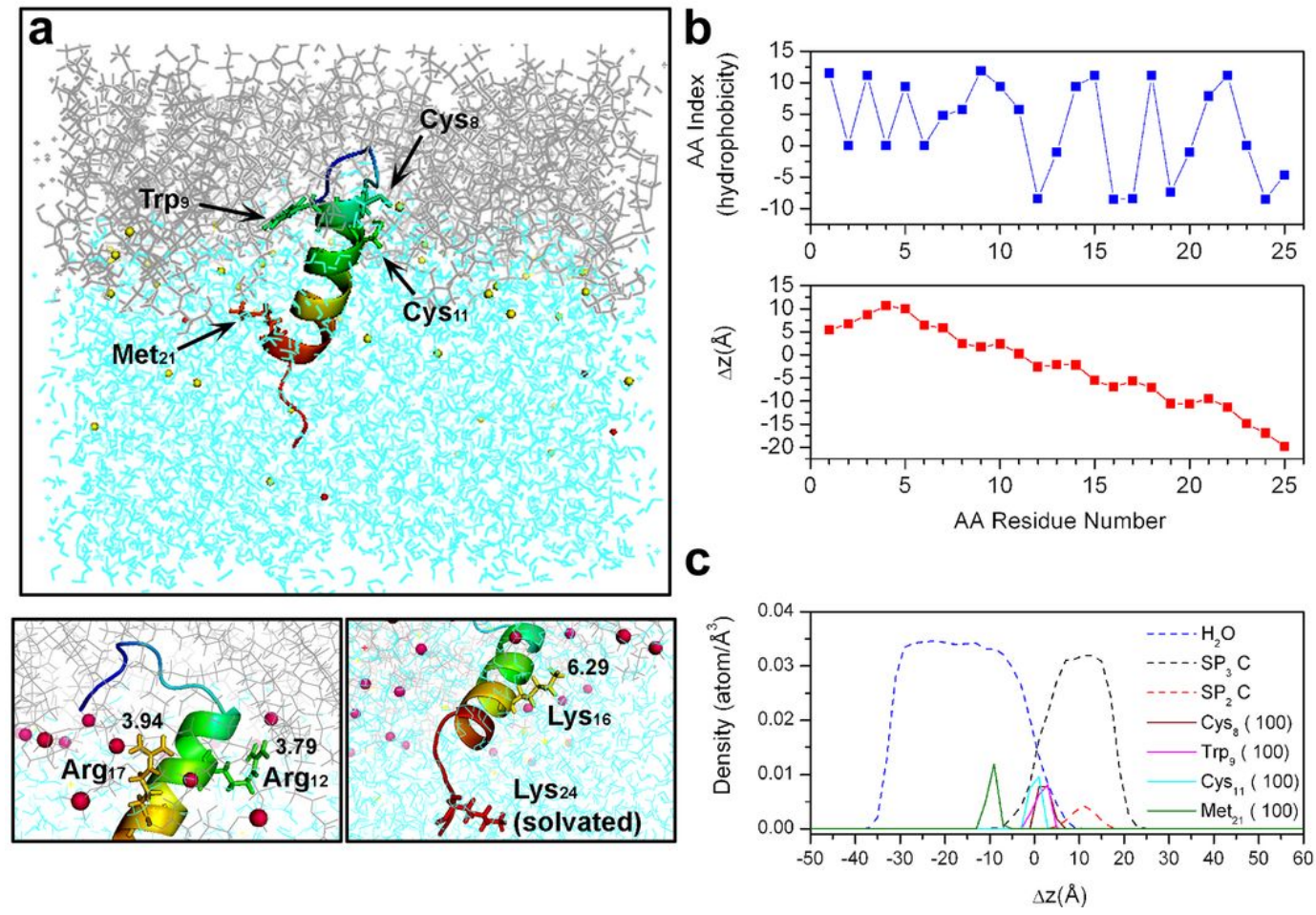

Figure 7.8: (a) Final snapshot after 2.0 ns MD simulation of SP-B B $_{1-25}$ in POPG monolayer at 60 $\AA^{2} /$ lipid is shown at top panel. The peptide is shown in rainbow color (C-terminal: red, N-terminal: blue), lipids in gray, water molecules in cyan, and chloride ions in red. Lower left and right show that Arg residues and Lys residues (displayed with sticks) are interacting with phosphate groups of lipids, respectively. (Phosphorous atoms are in magenta.) (b) AA index for hydrophobicity scale [46] (top) and $\Delta z$ of $\mathrm{C} \alpha$ of each residue averaged during the last $0.5 \mathrm{~ns}$ trajectory of 2.0 ns duration MD simulations (bottom) are plotted as a function of amino acid residue number. The air/water interface is located near $\Delta z=0$. (c) Atomic density profiles of SP- $\mathrm{B}_{1-25}$ in POG monolayer at 60 $\AA^{2} /$ lipid as a function of $\Delta z$ during the last $0.5 \mathrm{~ns}$ of the $2.0 \mathrm{~ns} \mathrm{MD}$ simulation. Blue dash line denotes the density profiles of oxygen atoms of water molecules. Black and red dash lines denote those of saturated and unsaturated carbons of lipid acyl chains, respectively. Wine, magenta, cyan, and olive solid lines denote the 100 times scaled density profiles of the $\mathrm{C}_{\alpha}$ carbon of $\mathrm{Cys}_{8}, \operatorname{Trp}_{9}$, $\mathrm{Cys}_{11}$, and Met 21 residues, respectively. 


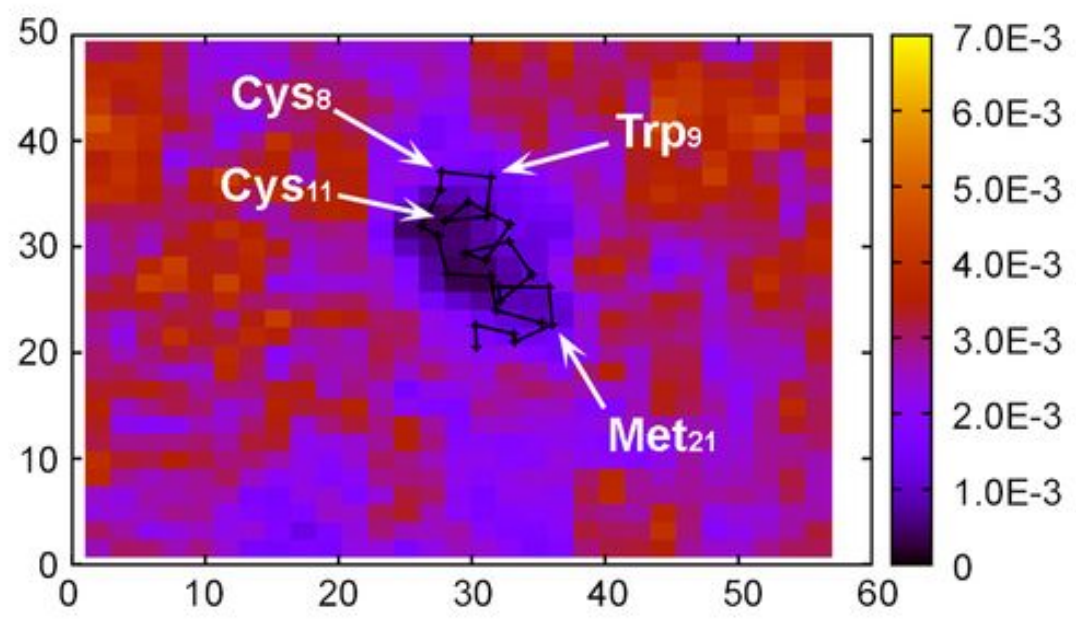

Figure 7.9: The $x y$-projected density profiles of saturated carbon atoms of lipid acyl chains is shown with colors and the averaged positions of $\mathrm{C}_{\alpha}$ carbons of $\mathrm{SP}^{-} \mathrm{B}_{1-25}$ in the POPG monolayer is shown with a black line (each residue is shown with cross).

oxidation.

Our findings present a detailed explanation for the mechanisms of the possible damage to the pulmonary system by ROS or direct ozone exposure. Further studies with a more elaborate model system comprising SP-B, SP-C, and various lipids could further clarify the effect of other environmental exposures, such as smoking and airborne particles, on the lung surfactant system.

\subsection{Acknowledgments}

The research described in this paper was carried out at the Beckman Institute and the Noyes Laboratory of Chemical Physics at the California Institute of Technology, the Computational NanoBioTechnology Laboratory at Georgia Institute of Technology and Jet Propulsion Laboratory under a contract with the National Aeronautics and Space Administration and funded through the Director's Research and Development Fund. We appreciate the support provided by the Beckman Institute Mass Spectrometry Resource Center. Partial support was also provided by the National Science Foundation (NSF) under grant No. CHE-0416381 (JLB, PI) and the National Cancer Institute under grant No. 5 U54 CA119347 (JRH, PI). 


\section{Bibliography}

[1] Anseth, J.; Goffin, A.; Fuller, G.; Ghio, A.; Kao, P. Am. J. Respir. Cell Mol. Biol. 2005, 33, 161-168.

[2] Halliwell, B.; Gutteridge, J. Biochem. J. 1984, 219, 1-14.

[3] Stansfield, A.; Jump, Z.; Sodlosky, S.; Rappaport, S.; Edelman, N.; Haldorsen, J.; Javed, T.; Martin, C.; Margulies, E. ed. Maple, D; American Lung Association National Headquarters: New York, 2008.

[4] Vangolde, L.; Batenburg, J.; Robertson, B. Physiol. Rev. 1988, 68, 374-455.

[5] Schram, V.; Hall, S. Biophys. J. 2004, 86, 3734-3743.

[6] Hawco, M.; Davis, P.; Keough, K. J. Appl. Physiol. 1981, 51, 509-515.

[7] Perez-Gil J.; Keough K. Biochim. Biophys. Acta-Mol. Basis Dis. 1998, 1408, 203-217.

[8] Clark J.; Wert S.; Bachurski C.; Stahlman M.; Stripp B.; Weaver T.; Whitsett J. Proc. Natl. Acad. Sci. U. S. A. 1995, 92, 7794-7798.

[9] Perez-Gil, J. Biophys. J. 2008, 94, 1542-1543.

[10] Pryor, W.; Das, B.; Church, D. Chem. Res. Toxicol. 1991, 4, 341-348.

[11] Uppu, R. M.; Cueto, R.; Squadrito, G. L.; Pryor, W. A. Arch. Biochem. Biophys. 1995, 319, $257-266$.

[12] Manzanares, D.; Rodriguez-Capote, K.; Liu, S. Y.; Haines, T.; Ramos, Y.; Zhao, L.; DohertyKirby, A.; Lajoie, G.; Possmayer, F. Biochemistry 2007, 46, 5604-5615.

[13] Andersson, S.; Kheiter, A.; Merritt, T. A. Lung 1999, 177, 179-189.

[14] Rodriguez-Capote, K.; Manzanares, D.; Haines, T.; Possmayer, F. Biophys. J. 2006, 90, 28082821.

[15] Grimm, R. L.; Hodyss, R.; Beauchamp, J. L. Anal. Chem. 2006, 78, 3800-3806. 
[16] Voss, L. F.; Hadad, C. M.; Allen, H. C. J. Phys. Chem. B 2006, 110, 19487-19490.

[17] Mundy, C. J.; Kuo, I. F. W. Chem. Rev. 2006, 106, 1282-1304.

[18] Enami, S.; Hoffmann, M. R.; Colussi, A. J. Proc. Natl. Acad. Sci. U. S. A. 2008, 105, 7365-7369.

[19] Enami, S.; Hoffmann, M. R.; Colussi, A. J. J. Phys. Chem. B 2008, 112, 4153-4156.

[20] Grimm, R. L.; Beauchamp, J. L. J. Phys. Chem. B 2003, 107, 14161-14163.

[21] Grimm, R. L.; Beauchamp, J. L. J. Phys. Chem. B 2005, 109, 8244-8250.

[22] Takamoto, D. Y.; Lipp, M. M.; von Nahmen, A.; Lee, K. Y. C.; Waring, A. J.; Zasadzinski, J. A. Biophys. J. 2001, 81, 153-169.

[23] Bruni, R.; Taeusch, H. W.; Waring, A. J. Proc. Natl. Acad. Sci. U. S. A. 1991, 88, 7451-7455.

[24] Longo, M. L.; Bisagno, A. M.; Zasadzinski, J. A. N.; Bruni, R.; Waring, A. J. Science 1993, $261,453-456$.

[25] MacKerell, A.D.; Bashford, D.; Bellott, M.; Dunbrack, R. L.; Evanseck, J. D.; Field, M. J.; Fischer, S.; Gao, J.; Guo, H.; Ha, S.; Joseph-McCarthy, D.; Kuchnir, L.; Kuczera, K.; Lau, F. T. K.; Mattos, C.; Michnick, S.; Ngo, T.; Nguyen, D. T.; Prodhom, B.; Reiher, W. E.; Roux, B.; Schlenkrich, M.; Smith, J. C.; Stote, R.; Straub, J.; Watanabe, M.; Wiorkiewicz-Kuczera, J.; Yin, D.; Karplus, M. J. Phys. Chem. B 1998, 102, 3586-3616.

[26] Plimpton, S. J. Comput. Phys. 1995, 117, 1-19.

[27] Hockney, R. W.; Eastwood, J. W. Computer simulation using particles; McGraw-Hill: New York, 1981.

[28] Karagulian, F.; Lea, A. S.; Dilbeck, C. W.; Finlayson-Pitts, B. J. Phys. Chem. Chem. Phys. 2008, 10, 528-541.

[29] Santrock, J.; Gorski, R. A.; Ogara, J. F. Chem. Res. Toxicol. 1992, 5, 134-141.

[30] Rivera, J. L.; Starr, F. W.; Paricaud, P.; Cummings, P. T. J. Chem. Phys. 2006, 125, 8.

[31] Ghosh, A.; Smits, M.; Bredenbeck, J.; Bonn, M. J. Am. Chem. Soc. 2007, 129, 9608.

[32] Lai, C. C.; Yang, S. H.; Finlayson-Pitts, B. J. Langmuir 1994, 10, 4637-4644.

[33] Pryor, W. A. Am. J. Clin. Nutr. 1991, 53, 702-722.

[34] Clauser, K. R.; Hall, S. C.; Smith, D. M.; Webb, J. W.; Andrews, L. E.; Tran, H. M.; Epstein, L. B.; Burlingame, A. L. Proc. Natl. Acad. Sci. U. S. A. 1995, 92, 5072-5076. 
[35] Seinfeld, J. H.; Pandis, S. N. Atmospheric chemistry and physics: From air pollution to climate change; John Wiley \& Sons: New York, 1998.

[36] von Gunten, U. Water Res. 2003, 37, 1443-1467.

[37] Pryor, W. A. Free Radic. Biol. Med. 1994, 17, 451-465.

[38] Pryor, W. A.; Uppu, R. M. J. Biol. Chem. 1993, 268, 3120-3126.

[39] Schoneich, C. BBA-Proteins Proteomics 2005, 1703, 111-119.

[40] Pryor, W. A.; Giamalva, D. H.; Church, D. F. J. Am. Chem. Soc. 1984, 106, 7094-7100.

[41] Berlett, B. S.; Stadtman, E. R. J. Biol. Chem. 1997, 272, 20313-20316.

[42] Buxton, G. V.; Greenstock, C. L.; Helman, W. P.; Ross, A. B. J. Phys. Chem. Ref. Data 1988, $17,513-886$.

[43] Kaznessis, Y. N.; Kim, S.; Larson, R. G. J. Mol. Biol. 2002, 322, 569-582.

[44] Baoukina, S.; Monticelli, L.; Risselada, H. J.; Marrink, S. J.; Tieleman, D. P. Proc. Natl. Acad. Sci. U. S. A. 2008, 105, 10803-10808.

[45] Kaznessis, Y. N.; Kim, S. T.; Larson, R. G. Biophys. J. 2002, 82, 1731-1742.

[46] Kawashima, S.; Ogata, H.; Kanehisa, M. Nucleic Acids Research 1999, 27, 368-369.

[47] Freites, J. A.; Choi, Y.; Tobias, D. J. Biophys. J. 2003, 84, 2169-2180.

[48] Gilliard, N.; Heldt, G. P.; Loredo, J.; Gasser, H.; Redl, H.; Merritt, T. A.; Spragg, R. G. J. Clin. Invest. 1994, 93, 2608-2615.

[49] Pryor, W. A. Free Radic. Biol. Med. 1992, 12, 83-88. 


\section{Appendix A}

\section{Discussions on Coarse-Graining of Time- and Length-Scale in Monte Carlo Simulations for AN-OPE SAM}

In order to connect from the atomistic quantum level to macroscopic level, it is necessary to coarse grain the system. We discuss below some of the consequences of the coarse graining on time- and length-scale.

\section{A.1 Time-Scale}

From the NN model, the energies required to initiate the P-to-T transition under 1.2 V/ $\AA$ and T-to-P transition without external field are $\Delta E^{\ddagger}=11.58 \mathrm{kcal} / \mathrm{mol}$ and $\Delta E^{\ddagger}=12.52 \mathrm{kcal} / \mathrm{mol}$, respectively. Assuming $\Delta S^{\ddagger}=k_{B} \ln (2500)$, from Eyring equation, $k=k_{B} T / h \exp \left(\Delta S^{\ddagger} / k_{B}\right) \exp \left(-\Delta E^{\ddagger} / k_{B} T\right)$, we obtain $\tau_{0}=1 / k=1.9 \times 10^{-8} \mathrm{~s}$ and $\tau_{0}=9.3 \times 10^{-8} \mathrm{~s}$ for P-to-T case and T-to-P case, respectively. By comparing these values with MC results of $\tau_{0}=274,193 \mathrm{MCS}$ and $\tau_{0}=357,135 \mathrm{MCS}$, we can estimate $1 \mathrm{MCS}$ as an order of $\sim 10^{-13} \mathrm{~s}$. Thus, in actual unit, the sweep rates of $1 \times 10^{-8} \mathrm{~V} / \mathrm{MCS}$, $4 \times 10^{-8} \mathrm{~V} / \mathrm{MCS}$, and $2 \times 10^{-7} \mathrm{~V} / \mathrm{MCS}$ are around $1 \times 10^{5} \mathrm{~V} / \mathrm{s}, 4 \times 10^{5} \mathrm{~V} / \mathrm{s}$, and $2 \times 10^{6} \mathrm{~V} / \mathrm{s}$, which are $\sim 10^{6}$ times faster than the experimental conditions.

\section{A.2 Length-Scale}

We found that in our simulations, the total time taken for the complete P-to-T transition under the constant external field of $F, t_{\mathrm{P} \text {-to- } \mathrm{T}}(F)$ is highly overestimated when $F$ is near $F_{c}$ due to the periodic boundary condition. It is a well known fact that the finite-size effect in simulations becomes serious near the critical point of phase transition [1]. This artifact makes the transition occur at once after 


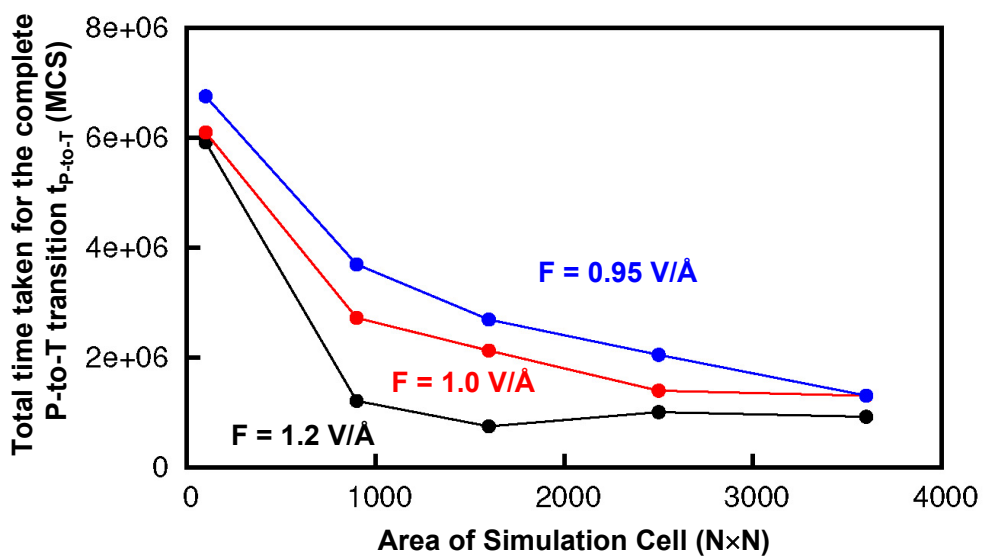

Figure A.1: Total time taken for the complete P-to-T transition, $t_{\mathrm{P}-\text {-to- } \mathrm{T}}$ by varying the area of the periodic simulation cell as $10 \times 10=100,30 \times 30=900,40 \times 40=1600,50 \times 50=2500$, and $60 \times 60=3600$. Black line denotes the change of $t_{\mathrm{P} \text {-to- } \mathrm{T}}$ under the external field of $F=1.2 \mathrm{~V} / \AA$, red line denotes the change of $t_{\mathrm{P} \text {-to- } \mathrm{T}}$ scaled by 10 under the external field of $F=1.0 \mathrm{~V} / \AA$, and blue line denotes the change of $t_{\mathrm{P}-\text { to- } \mathrm{T}}$ scaled by 15 under the external field of $F=0.95 \mathrm{~V} / \AA$. The $t_{\mathrm{P} \text {-to- } \mathrm{T}}$ value is converged at the area of $900-1600$ and $2500-3600$ when $F=1.2 \mathrm{~V} / \AA$ and $1.0 \mathrm{~V} / \AA$, respectively. When $F=0.95 \mathrm{~V} / \AA$, the $t_{\mathrm{P}-\text { to- } \mathrm{T}}$ value is not converged until the area of 3600 . This infers that we need much larger simulation cell to get the right converged value of $t_{\mathrm{P} \text {-to- } \mathrm{T}}$ as the $F$ approaches to the critical field, $F_{c}=0.56 \mathrm{~V} / \AA$. Otherwise, we will get the overestimated value of $t_{\mathrm{P}-\mathrm{to}-\mathrm{T}}$ near $F_{c}$.

the bias voltage is increased sufficiently, and finally yields a narrower range of NDR (Figure A.1). 


\section{Appendix B}

\section{Effect of Molecular Fluctuations on the Electrical Conductivity of AN-OPE SAM}

Electrical conductivity through the AN-OPE is estimated using the fitting equation of Figure 2.8 based on the twisting angles, $\chi_{1}$ and $\chi_{2}$, from MD simulations with $(10 \times 10)$ unit cell. We mimicked two sets of voltage sweep cycle by following MD procedures:

(a) QM optimized P structure is employed for the initial structure, then, annealed in the absence of external field. The conformations are almost co-planar as predicted from QM calculation with a well-developed HB network. Estimated conductivity is large with the averaged value of 0.035 .

(b) External field of $1.2 \mathrm{~V} / \AA$ is turned on to the final structure of (a). The SAM structure is partially disordered and $\chi_{1}$ has largest probability at $90^{\circ}$. Estimated conductivity 3.21 times decreases to 0.011 .

(c) QM optimized T structure is employed for the initial structure, then, annealed under the external field of $1.2 \mathrm{~V} / \AA$ A. Structural fluctuation allows AN-OPEs to have $\sim 90^{\circ}$ twisted conformations, resulting the loss of HB network. SAM is well-ordered. Estimated conductivity remains same to (b) with the value of 0.011 .

(d) External field is turned down from the final structure of (a). SAM forms a pretty disordered structure and $\chi_{1}$ has no strongly preferring angle. Estimated conductivity 1.23 times increases to 0.013 .

(e) The final structure from step (d) is annealed in the absence of external field. SAM still has disordered structure, however, probability at $\chi_{1}=90^{\circ}$ slightly decreases. Estimated conductivity is slightly increased to 0.014 . 
(f) External field of $1.2 \mathrm{~V} / \AA$ is re-turned on to the final structure of (e). Structure and twisting angle is similar to (b), however, it is a bit more disordered. Thus, estimated conductivity is a bit smaller than (b) with the value of 0.010 .

(g) The final structure from step (d) is annealed under the external field of $1.2 \mathrm{~V} / \AA$, and the SAM has still similar structure to (f). Estimated conductivity remains same to (f) with the value of 0.010 .

(h) External field is re-turned down from the final structure of (g). SAM forms a more disordered structure to (d). Thus, estimated conductivity is smaller than (d) with the value of 0.012 .

(i) The final structure from step (h) is annealed in the absence of external field. SAM still has a disordered structure, however, probability at $\chi_{1}=90^{\circ}$ slightly decreases. Estimated conductivity is 1.05 times increased to 0.013 .

The conductivities during the second cycle (f,g,h,i) shows $\sim 6 \%$ smaller values compared to the conductivities during the first cycle $(\mathrm{b}, \mathrm{c}, \mathrm{d}, \mathrm{e})$. These results are shown at Figure B.1. 

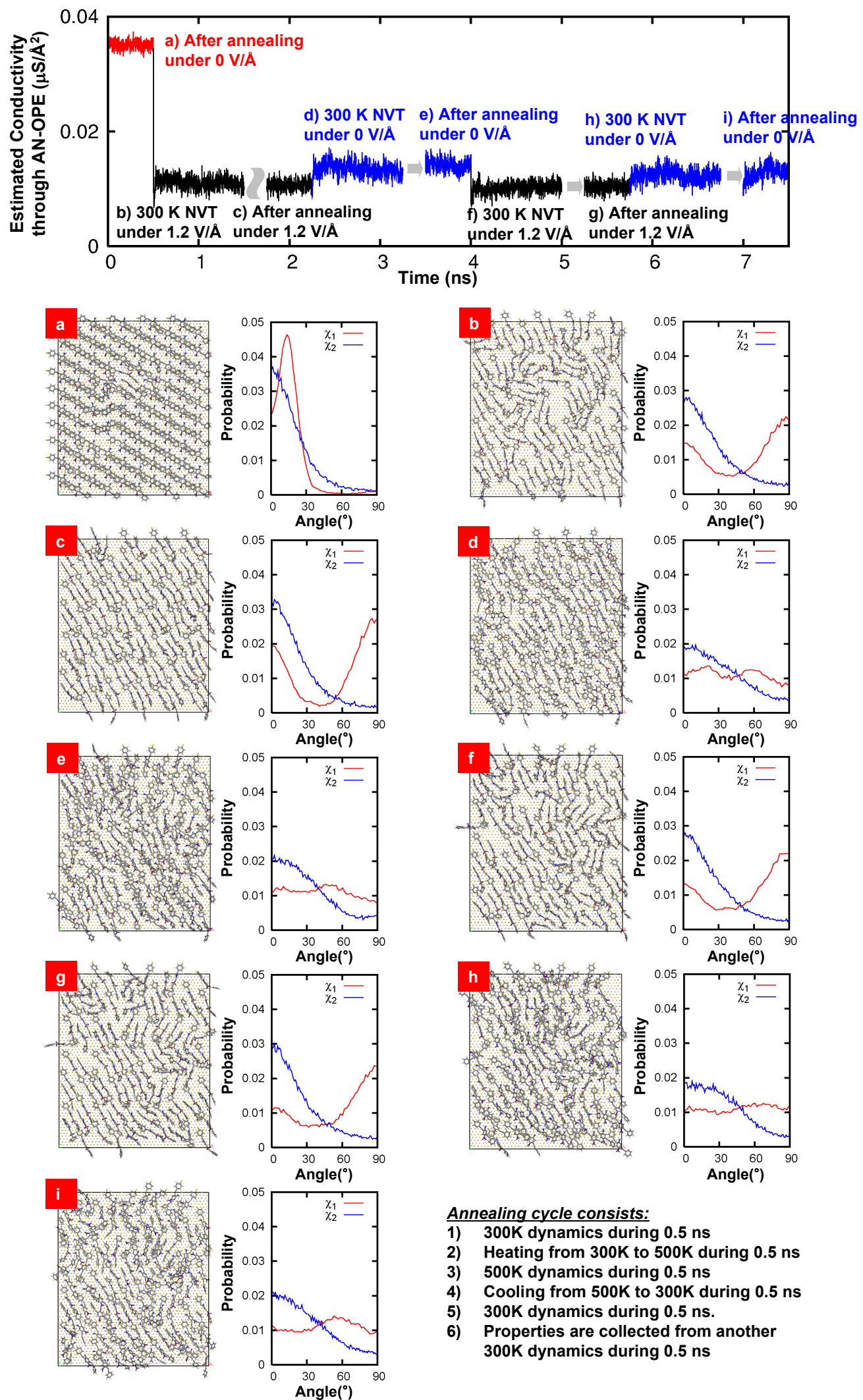

Annealing cycle consists:

1) $300 \mathrm{~K}$ dynamics during $0.5 \mathrm{~ns}$

2) Heating from $300 \mathrm{~K}$ to $500 \mathrm{~K}$ during $0.5 \mathrm{~ns}$

3) $500 \mathrm{~K}$ dynamics during $0.5 \mathrm{~ns}$

4) Cooling from $500 \mathrm{~K}$ to $300 \mathrm{~K}$ during $0.5 \mathrm{~ns}$

5) $300 \mathrm{~K}$ dynamics during $0.5 \mathrm{~ns}$.

6) Properties are collected from another

$300 \mathrm{~K}$ dynamics during $0.5 \mathrm{~ns}$

Figure B.1: (top panel) Electrical conductivity through the AN-OPE is estimated based on the twisting angles from MD simulations with $(10 \times 10)$ unit cell. (bottom panels) The final snapshot after each step is shown with the populations of $\chi_{1}$ and $\chi_{2}$, which are averaged during the last 0.5 ns dynamics. 


\section{Appendix C}

\section{NDR in Other OPE-derivative Systems}

We investigated that how our mechanism predicts the possibility of NDR in other systems containing other OPE derivatives.

\section{C.1 Bare OPE}

For bare OPE (B-OPE) with no functional groups our mechanism would suggest that there is no NDR effect, since the lack of functional groups leads to only a small molecular dipole moment to interact with the external field and there is no mechanism for the increased applied field to convert from the high conductance $\mathrm{P}$ phase to the low conductance $\mathrm{T}$ phase. Reference [2] shows the experimental data on B-OPE, which shows no NDR effect.

\section{C.2 Nitro OPE}

We calculated that the SAM of 5-nitro monosubstituted OPE system (N-OPE) on Au (111) surface. We figured out that N-OPE also can have two conformations of $\mathrm{P}$ and $\mathrm{T}$ on the SAM by forming weak HB networks along [1120] direction and [1010] direction, respectively. The lack of amino group makes $\mathrm{NO}_{2}$ form a weak $\mathrm{HB}$ with the positive aromatic $\mathrm{H}$ atoms of the adjacent molecules (Figure C.1).

The twist angles are $\chi=4^{\circ}$ and $\chi=163^{\circ}$ and the heights of the terminal ring are $8.73 \AA$ and 7.63 $\AA$ for $\mathrm{P}$ and $\mathrm{T}$, respectively. These are almost identical to the values in AN-OPE case. Therefore, we expect the conductance ratio for N-OPE to be very similar to AN-OPE.

From PBE DFT calculations, $\mathrm{P}$ is more stable than $\mathrm{T}$ by $6.57 \mathrm{kcal} / \mathrm{mol}$ when no external field is applied. The dipole moment components in the [0001] direction are 3.83 (2.01) debye for $\mathrm{P}$ and 6.61 (3.86) debye for $\mathrm{T}$ from the analysis of Mulliken charges, while the parenthetical values are 
from quantum mechanical wave-function. Thus, the favorable state is changed from $\mathrm{P}$ to $\mathrm{T}$ as the external field is applied (Figure C.2). This suggests that the N-OPE also undergoes conformational change from $\mathrm{P}$ to $\mathrm{T}$ as the bias voltage increases. The critical field $F_{c}$ is calculated as $0.49 \mathrm{~V} / \AA$ and $0.74 \mathrm{~V} / \AA$ when using dipole moments from Mulliken charge and QM wave-function, respectively. Since these are in the range of the $F_{c}$ in AN-OPE system $(0.56 \mathrm{~V} / \AA)$, the critical bias voltage where NDR occurs is similar to the critical bias voltage of AN-OPE system of $\sim 0.6 \mathrm{~V}$. Reference [2] also shows the experimental data on $\mathrm{N}-\mathrm{OPE}$, which shows a well-defined hysteretic NDR at $\sim 0.6 \mathrm{~V}$ as predicted from our calculations. 


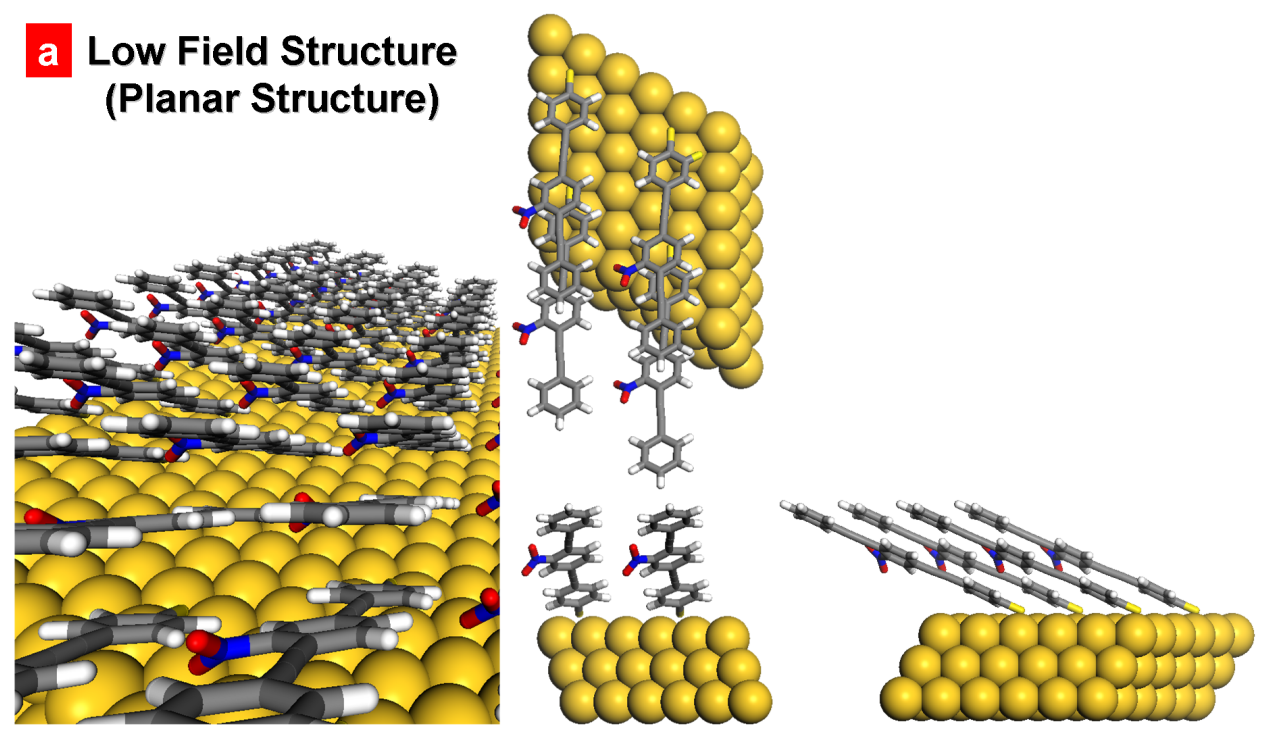

\section{b High Field Structure (Twisted Structure)}
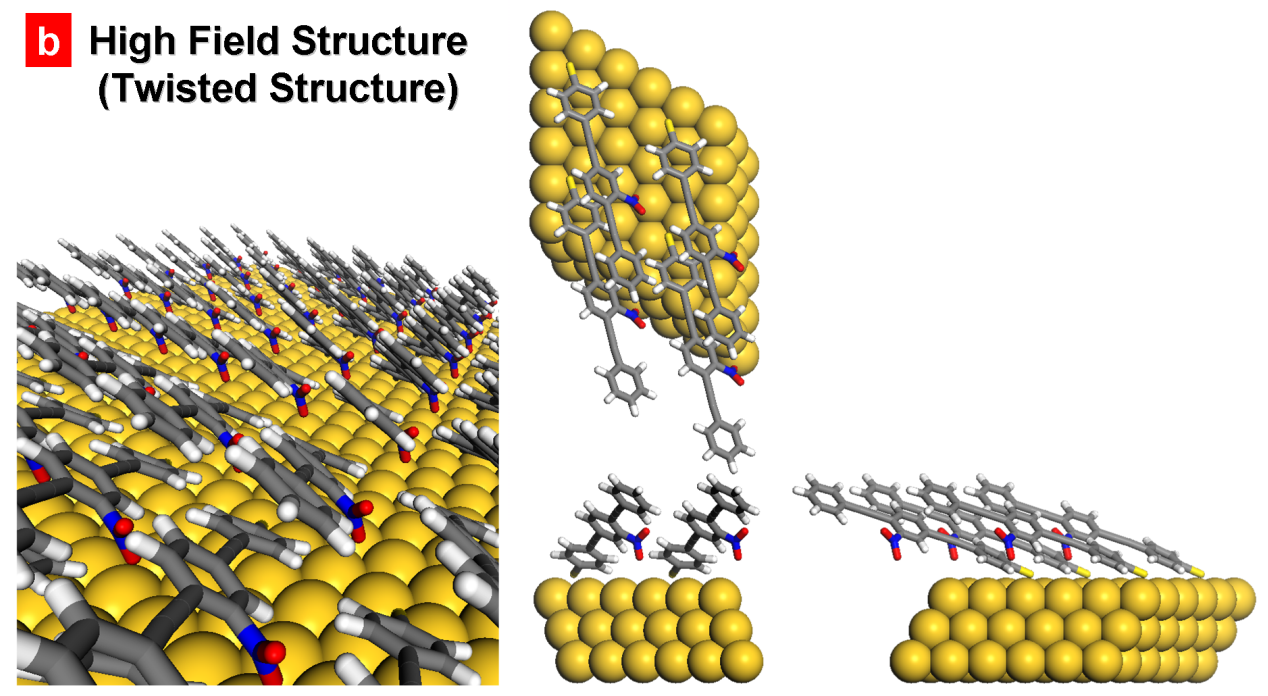

Figure C.1: (a) Optimized geometry for the low field structure (P) of N-OPE SAM. Here [0001] is the surface normal and the views are along $z$-axis (upper middle), $y$-axis (lower middle), and $x$-axis (lower right). The left picture is a perspective along the axis of one plane of molecules. The weak hydrogen bonding network is aligned along the [1120] direction. (b) Optimized geometry for the high field structure (T) of N-OPE SAM. Here [0001] is the surface normal and the views are along $z$-axis (upper middle), $y$-axis (lower middle), and $x$-axis (lower right). The left picture is a perspective along the axis of one plane of molecules. The weak hydrogen bonding network is aligned along the [10̄0] direction. 


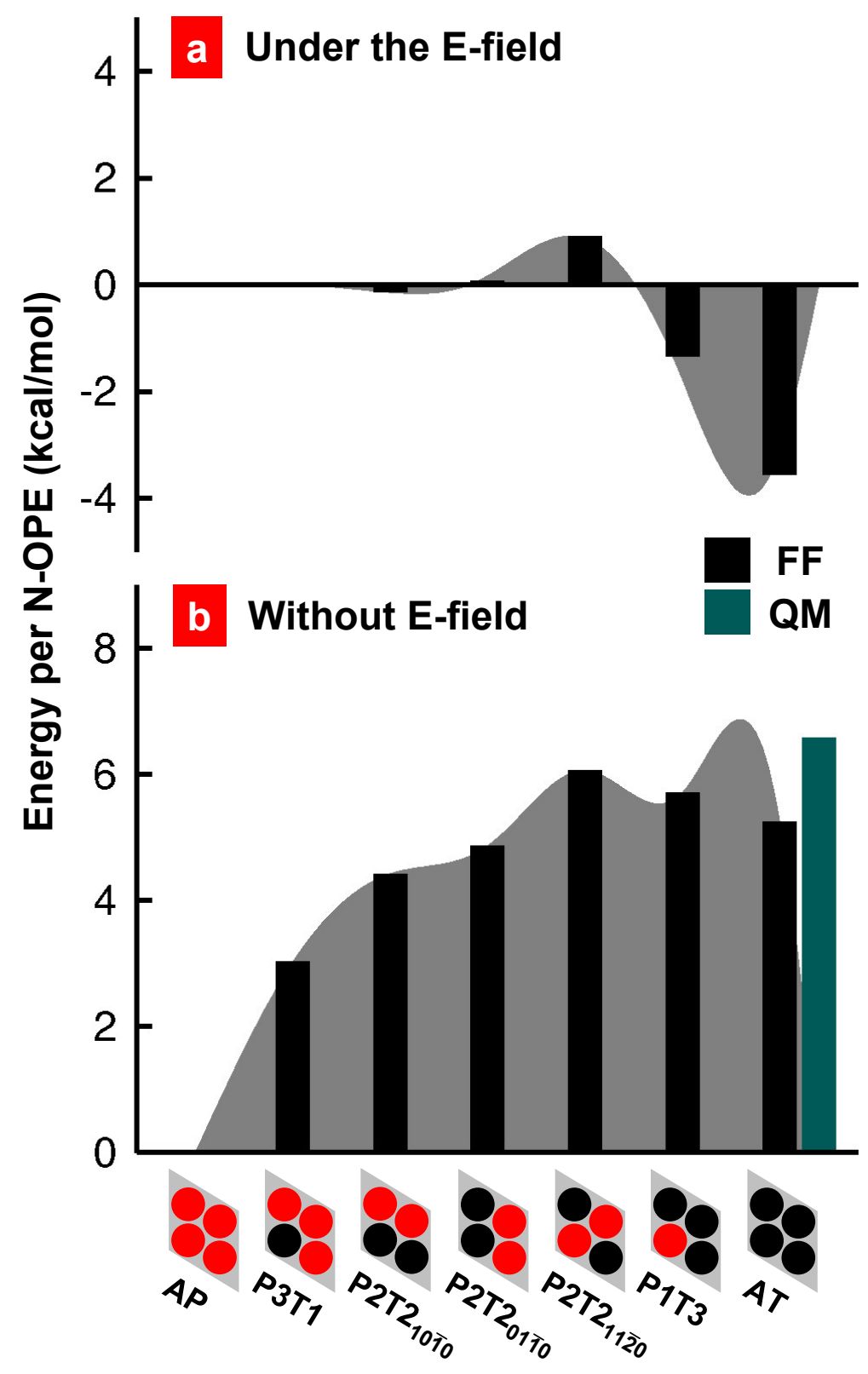

Figure C.2: (a) Energies of various conformations of N-OPE relative to the AP conformation computed with a $0.6 \mathrm{~V} / \AA$ external field from FF calculations (black histograms). (b) Energies of various conformations of N-OPE relative to the AP conformation computed with no external field from $\mathrm{FF}$ calculations (black histograms), and QM calculations (green histograms). 


\section{Appendix D}

\section{Conversion Factor between External Electric Field and Bias Voltage}

To connect the NEGF results and MC simulations, we need to convert the magnitude of external field, $F$, into the bias voltage, $V$. Since the critical bias voltage $V_{c}$ is observed as $\sim 0.6 \mathrm{~V}$ from the experiment and the critical field $F_{c}$ is $0.56 \mathrm{~V} / \AA$ in our NN model, we set the linear coefficient $c$ as $0.93 \AA^{-1}$, which is defined by $F / V$. 


\section{Appendix E}

\section{Mulliken Charge Distributions of Bistable [2]Rotaxane Molecular Switch Depending on CBPQT ${ }^{4+}$ Ring's Position}

The shuttling motion of charge accepting cyclobis-(paraquat- $p$-phenylene) $\left(\mathrm{CBPQT}^{4+}\right)$ between the charge donating stations such as tetrathiafulvalene (TTF) and 1,5-dioxynaphthalene (DNP) moieties is governed by the charge transfer amount between the ring and the backbone. Since the potential energy surface that the $\mathrm{CBPQT}^{4+}$ ring suffers while traveling along the backbone has a large dependency on the local charge distribution of the rotaxane molecule, we performed DFT calculations for 9 different rings' positions by varying the oxidation states, $0,+1$, and +2 . Computed Mulliken charge distributions are tabulated in this section. 


\section{a) Backbone}

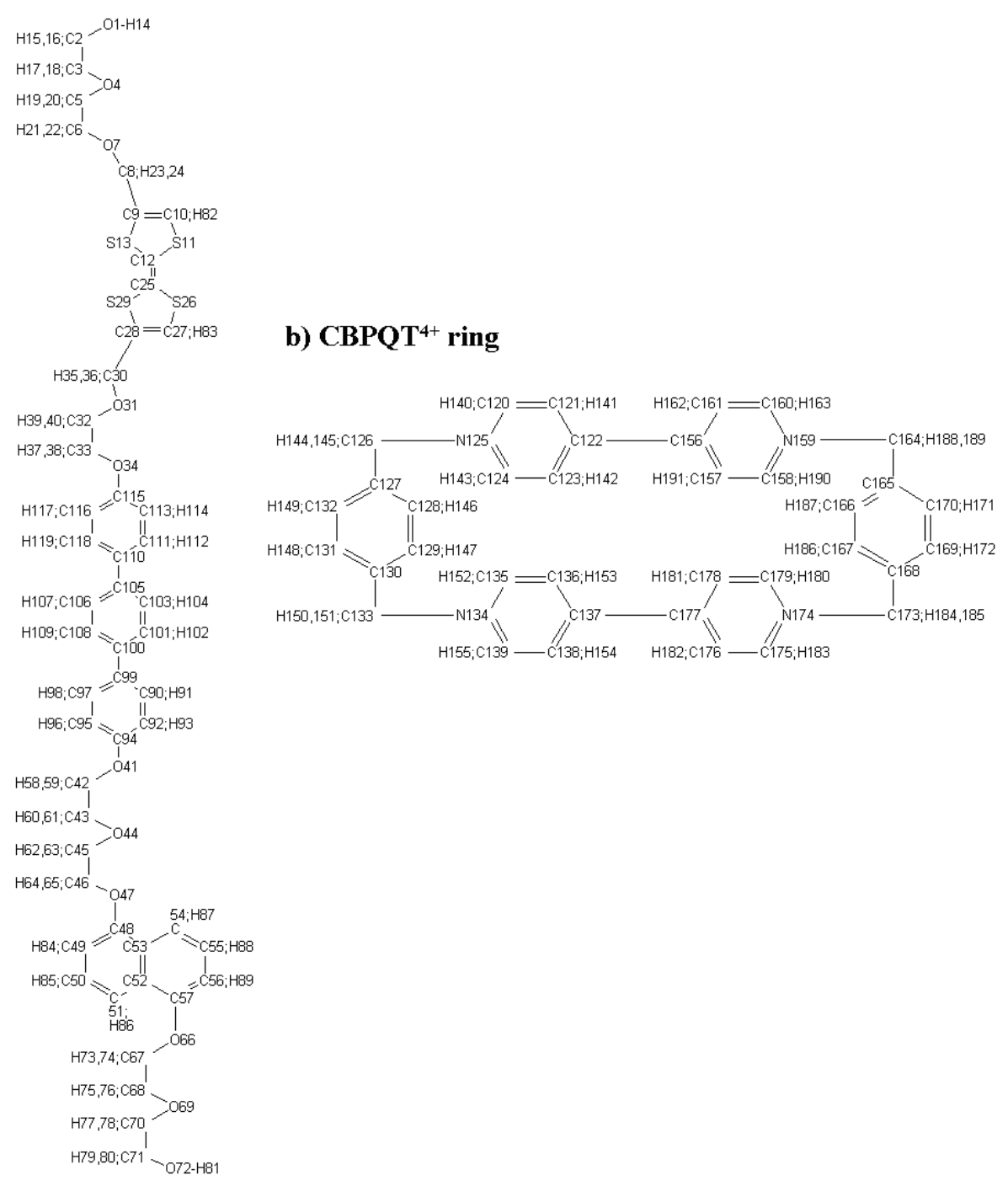

Figure E.1: Structures of a) backbone part and b) $\mathrm{CBPQT}^{4+}$ ring part with numbered atoms. The assigned numbers on the atoms are associated with the partial charge distribution data shown in Tables E.1, E.2, and E.3. 
Table E.1: Partial Charge Distribution of Neutral Rotaxane when the Center of Mass of the $\mathrm{CBPQT}^{4+}$ Ring Moves from $z=10.92 \AA$ (TTF side) to $z=44.83 \AA$ (DNP side)

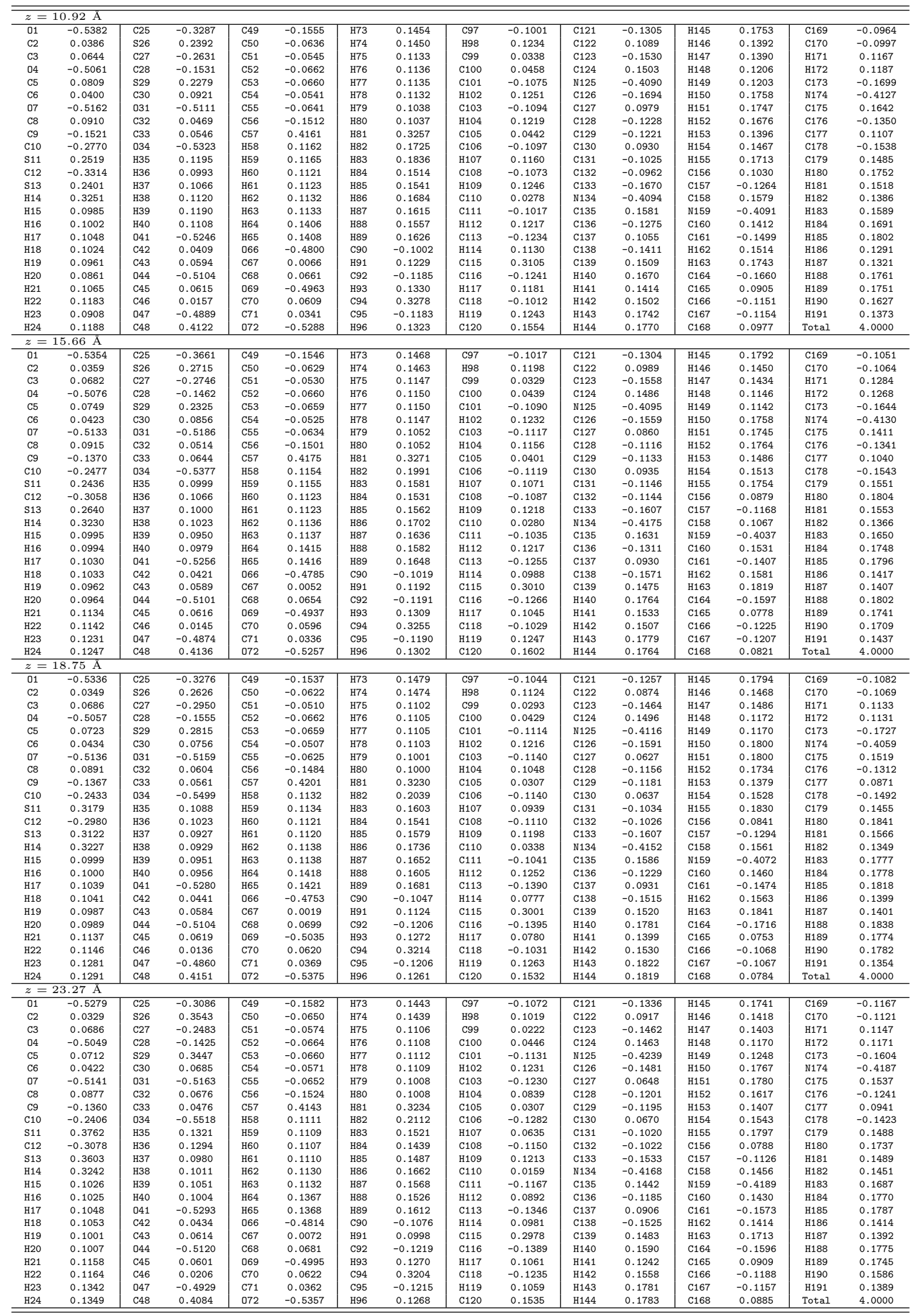


151

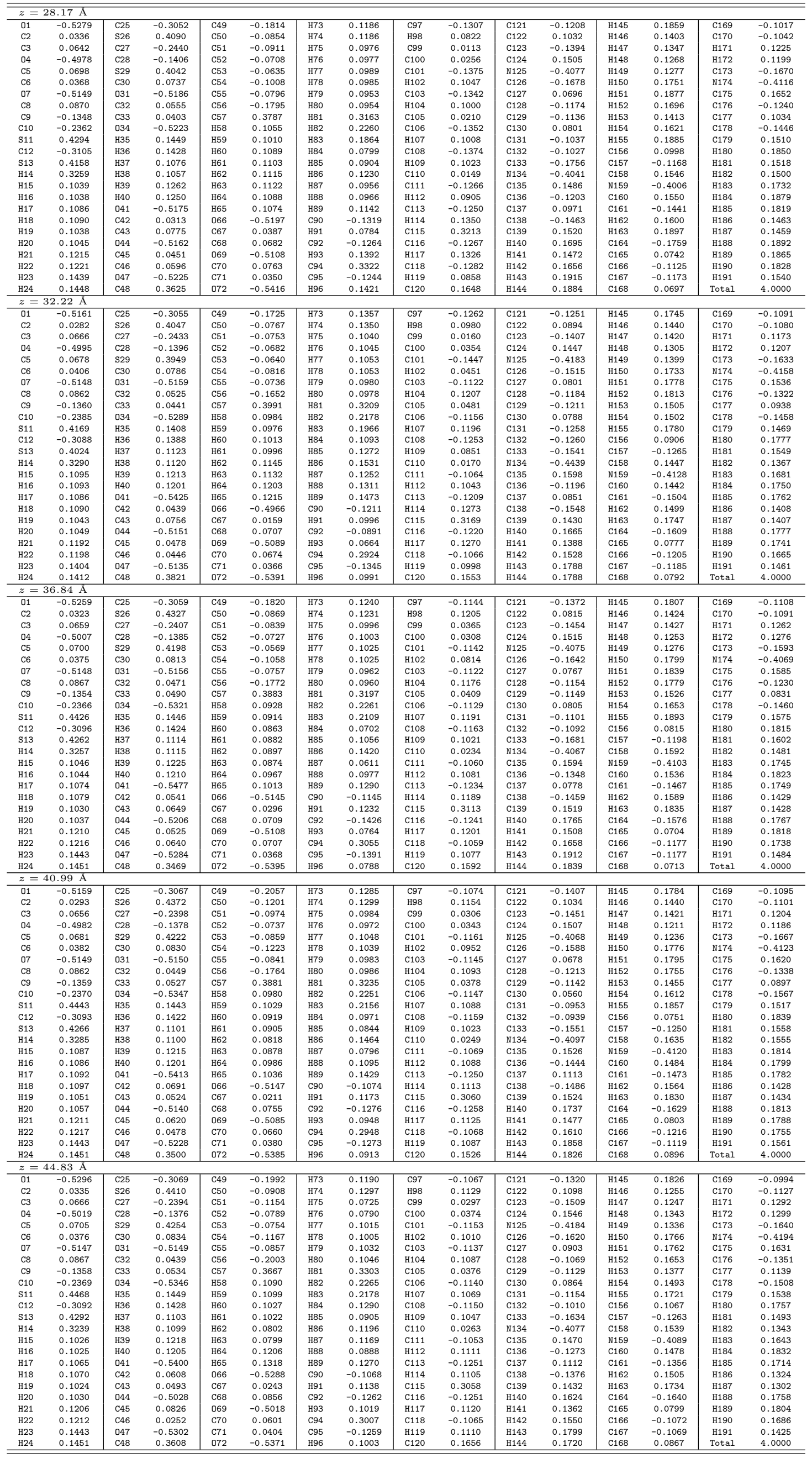


Table E.2: Partial Charge Distribution of +1 Oxidized Rotaxane when the Center of Mass of the $\mathrm{CBPQT}^{4+}$ Ring Moves from $z=10.92 \AA$ (TTF side) to $z=44.83 \AA$ (DNP side)

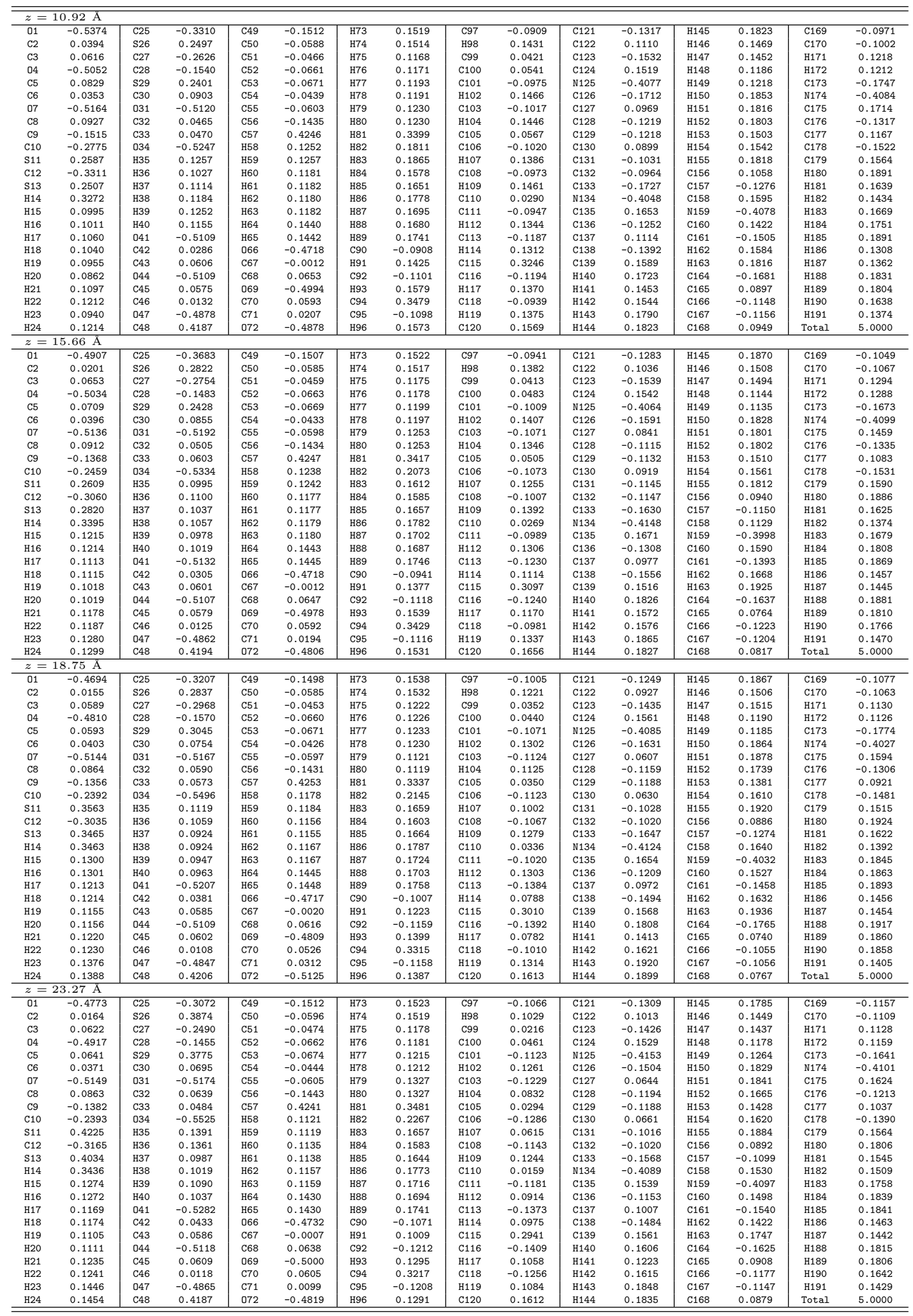


153

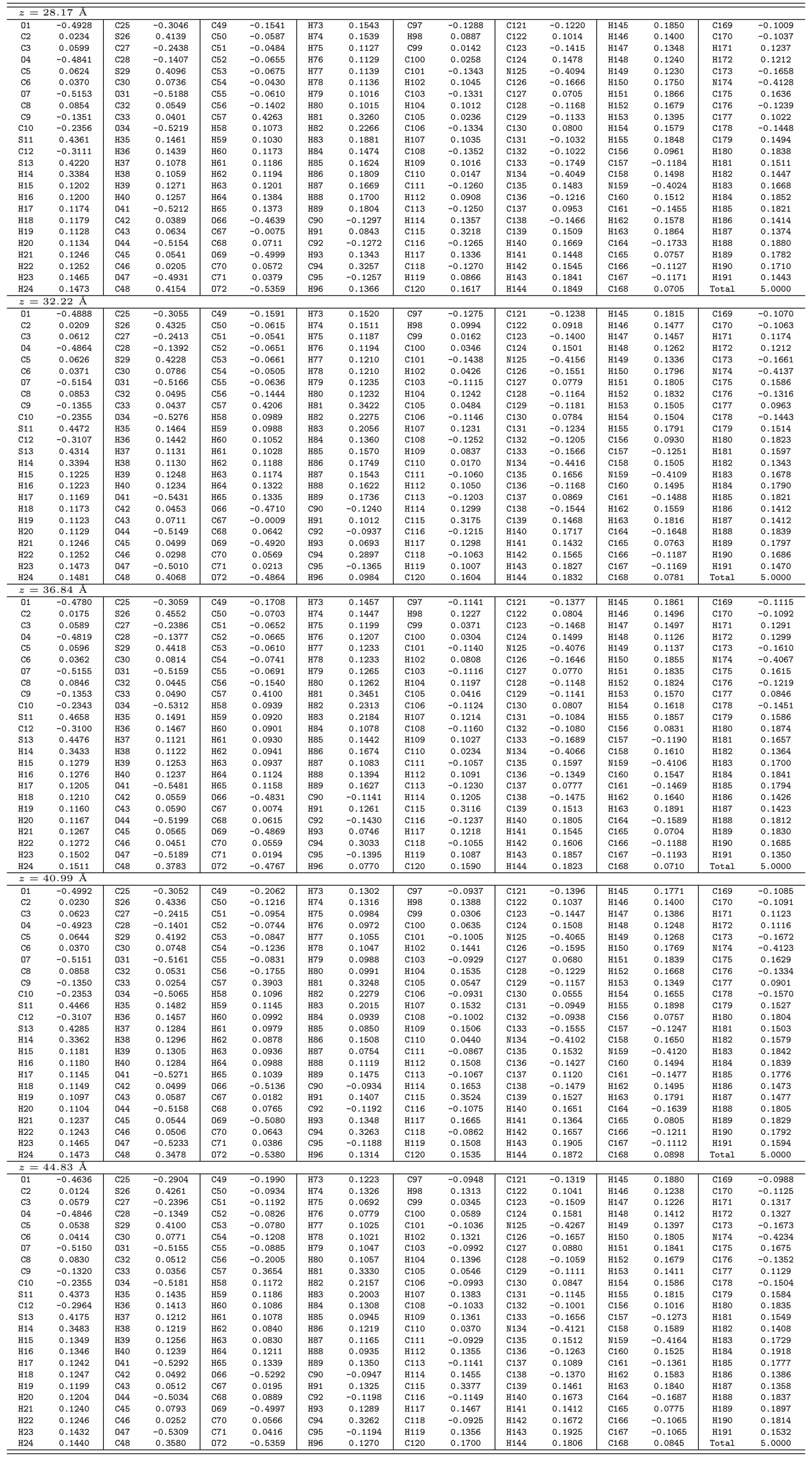


Table E.3: Partial Charge Distribution of +2 Oxidized Rotaxane when the Center of Mass of the $\mathrm{CBPQT}^{4+}$ Ring Moves from $z=10.92 \AA$ (TTF side) to $z=44.83 \AA$ (DNP side)

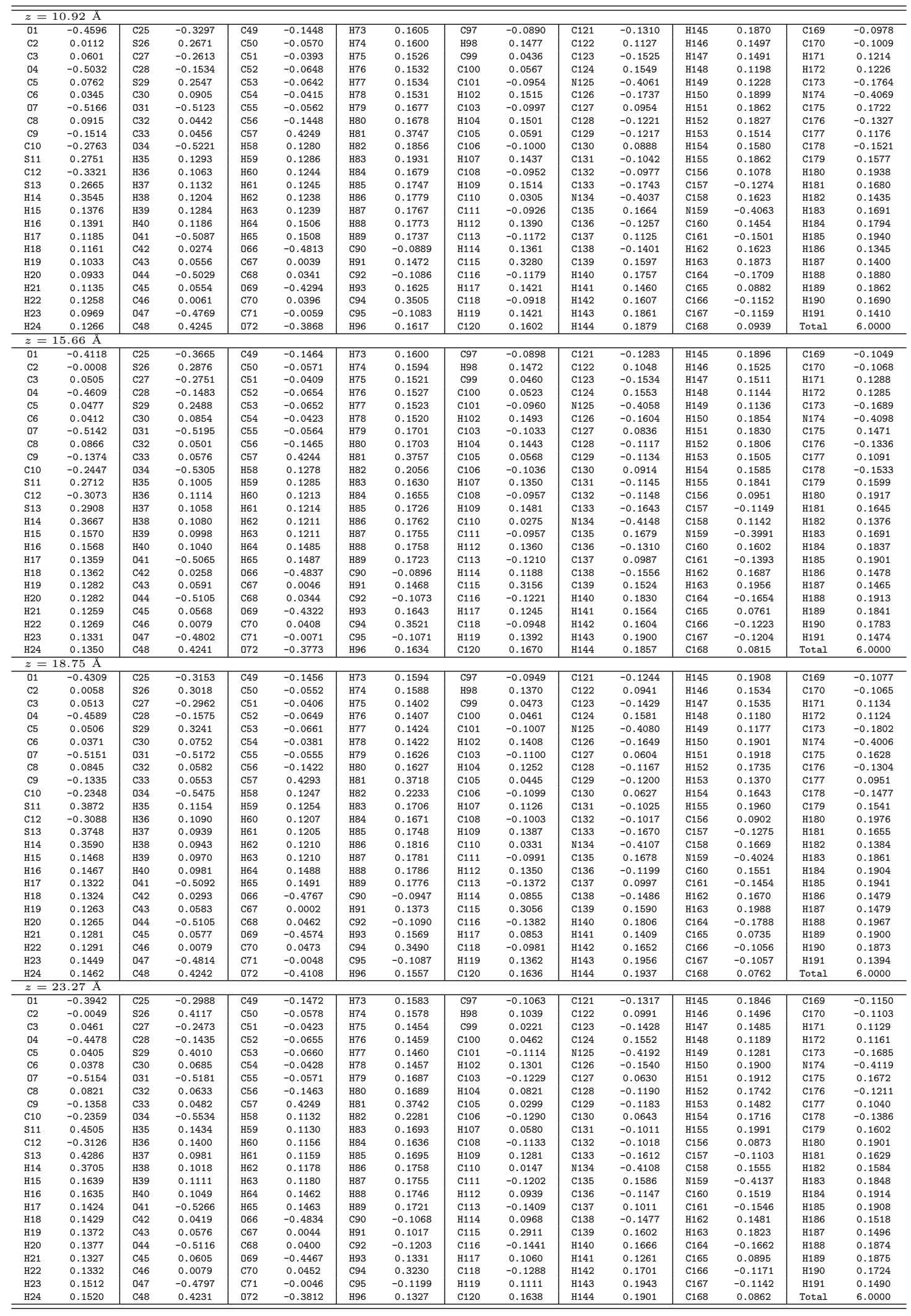


155

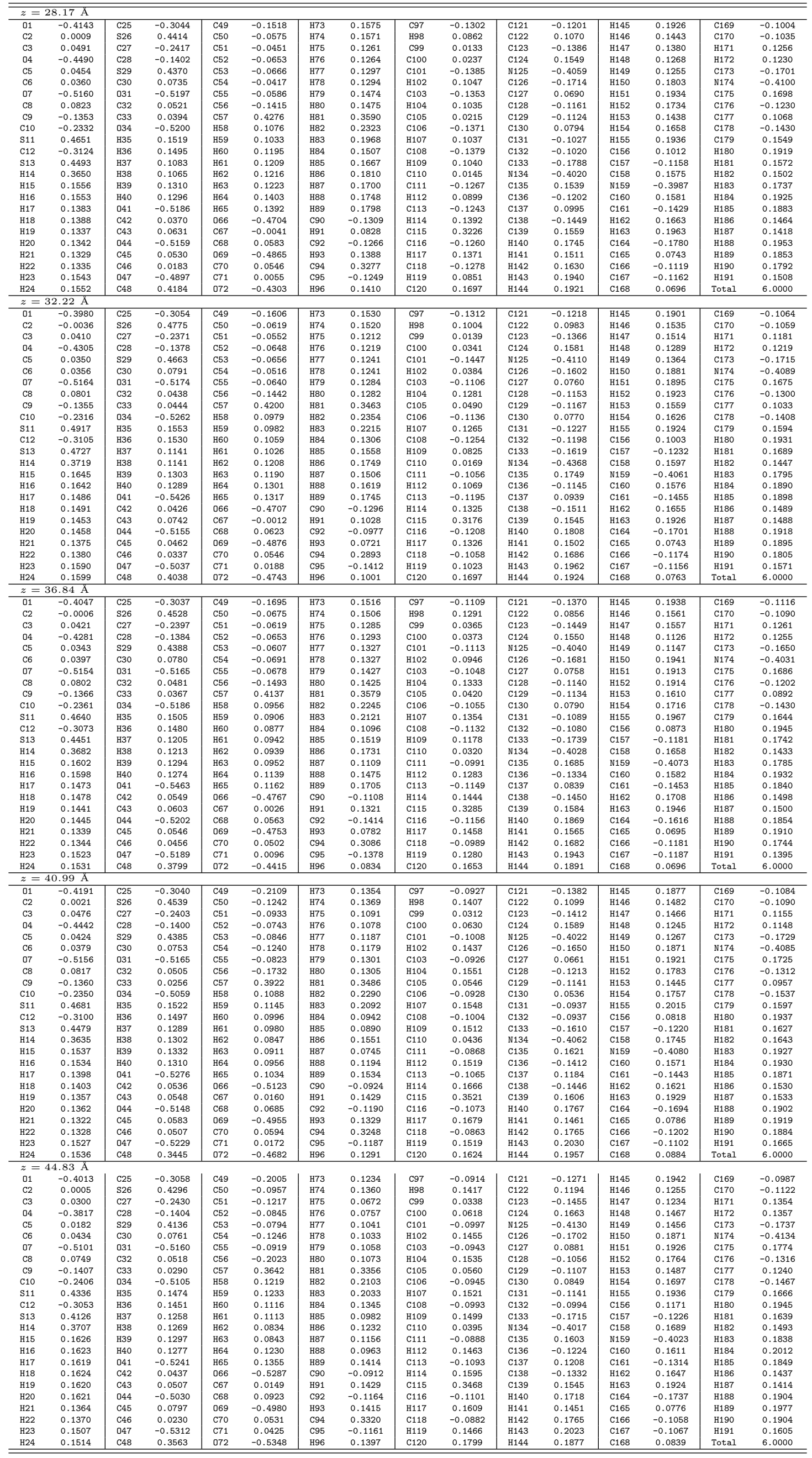




\section{Appendix $\mathbf{F}$}

\section{Consideration of Metric Effect on the Bistable [2/Rotaxane Molecule during the Constant MD Simulations using Fixman's Theorem}

When we consider a system with $N$ generalized coordinates $q_{i}$, associated conjugated momenta $p_{i}$, and masses $m_{i}$, the Hamiltonian of this system $H$ is given by

$$
H(p, q)=\frac{1}{2} \mathbf{p}^{T} \mathbf{M}^{-1} \mathbf{p}+V(\mathbf{q}),
$$

where $\mathbf{M}$ is the "symmetric metric tensor" or "mass-metric tensor" that is defined as

$$
M_{k l}=\sum_{i}^{N} m_{i} \frac{\partial \mathbf{r}_{i}}{\partial q_{k}} \cdot \frac{\partial \mathbf{r}_{i}}{\partial q_{l}}
$$

Among $N$ generalized coordinates, we consider that $\nu$ generalized coordinates $q_{i}^{\prime \prime}$ are constrained, and rests of them $q_{i}^{\prime}$ are not. Then, according to the Fixman's theorem [3], the ratio of the original partition function $Q$ and the partition function from the constraint dynamics $Q_{c}$ is

$$
\frac{Q}{Q_{c}}=(\operatorname{det} \mathbf{Z})^{-1 / 2}
$$

where

$$
Z_{k l}=\sum_{i}^{\nu} \frac{1}{m_{i}} \frac{\partial q_{k}^{\prime \prime}}{\partial \mathbf{r}_{i}} \cdot \frac{\partial q_{l}^{\prime \prime}}{\partial \mathbf{r}_{i}}
$$

In our simulation, the ( $\operatorname{det} \mathbf{Z})$ is determined as the inverse of the $\mathrm{CBPQT}^{4+}$ ring's mass, $1 / M_{\text {ring }}$. From Equation F.3, the difference between the original free energy $F=-k_{B} T \ln Q$ and the free 
energy from the constraint dynamics $F_{c}=-k_{B} T \ln Q$ is determined by

$$
F-F_{c}=-k_{B} T \ln (\operatorname{det} \mathbf{Z})^{-1 / 2}=-\frac{1}{2} k_{B} T \ln M_{\text {ring }}=\text { const. }
$$

Therefore, the metric effect only adds a constant scalar value to the absolute free energy values, which has no influence on the relative energetics. 


\section{Appendix G}

\section{Time for Consumption of POPG}

The formation of primary ozonide (POZ), which is the first step in the ozonolysis of 1-palmitoyl-2oleoyl-sn-phosphatidylglycerol (POPG) at the air-liquid interface, is described as

$$
\mathrm{O}_{3}+\mathrm{POPG} \stackrel{k_{1}}{\longrightarrow} \mathrm{POZ}
$$

The ozone concentration is assumed to be constant during the reaction, which allows calculating the reaction rate using the pseudo-first order rate constant $k_{2}=k_{1}\left[\mathrm{O}_{3}\right]$, where $k_{1}=4.5 \times 10^{-16}$ $\mathrm{cm}^{3}$ molecule ${ }^{-1} \mathrm{~s}^{-1}$ adopted from the OPPC ozonolysis on $\mathrm{NaCl}[4]$. The ozone concentration, measured spectrophotometrically using an absorption cell with $10 \mathrm{~cm}$ path length, is determined to be $\sim 5 \times 10^{14}$ molecule $\mathrm{cm}^{-3}$ (20 ppm). The reaction rate is expressed as

$$
-\frac{d[\mathrm{POPG}]_{\text {surf }}}{d t}=k_{2}[\mathrm{POPG}]_{\text {surf }, 0} .
$$

Solving Equation G.2 gives

$$
\frac{[\mathrm{POPG}]_{\text {surf }}}{[\mathrm{POPG}]_{\text {surf }, 0}}=e^{-k_{2} t}
$$

For $90 \%$ and $99 \%$ depletion of POPG at the air-liquid interface, it takes $\sim 10 \mathrm{~s}$ and $\sim 20 \mathrm{~s}$, respectively. 


\section{Appendix $\mathbf{H}$}

\section{Bulk-phase Ozonolysis}

\section{H.1 Methods}

\section{H.1.1 Chemicals and Reagents}

Ammonium bicarbonate $\left(\mathrm{NH}_{4} \mathrm{HCO}_{3}\right)$, iron (II) dichloride $\left(\mathrm{FeCl}_{2}\right)$, sodium ethylenediaminetetraacetic acid (EDTA), trypsin from porcine pancreas were purchased from Sigma-Aldrich (St. Louis, MO). SP-B $1-25$ was purchased from Biomer Technology (Hayward, CA).

\section{H.1.2 Bulk-phase $\mathrm{O}_{3}$ Reaction}

A continuous flow of $\sim 20 \mathrm{ppm}_{3}$ in air was applied to the $100 \mu \mathrm{M}$ SP-B $1-25$ solution in 1:1 (by volume) water and methanol solvent for $30 \mathrm{~s}, 1,1.5,2,3,4$, and 5 min. The SP-B ${ }_{1-25}$ solution was diluted to $50 \mu \mathrm{M}$ for ESI with 1:1 water/methanol and $1 \%$ acetic acid by volume. Trypsin digests of $\mathrm{SP}-\mathrm{B}_{1-25}$ and $\mathrm{O}_{3}$ treated $\mathrm{SP}_{-} \mathrm{B}_{1-25}$ were prepared by incubating $200 \mu \mathrm{M}$ of SP-B $\mathrm{B}_{1-25}$ with $6 \mu \mathrm{g}$ of trypsin from porcine pancreas in $1 \mathrm{~mL}$ of water containing $25 \mathrm{mM}$ ammonium bicarbonate $\left(\mathrm{NH}_{4} \mathrm{HCO}_{3}\right)$ at $37^{\circ} \mathrm{C}$ for 4 hours. The trypsin was then removed using a Millipore Microcon centrifugal filter fitted with an Ultracel YM-10 membrane. The sample solution was diluted to an appropriate concentration for ESI with 1:1 water/methanol and $1 \%$ acetic acid by volume. Product analysis was performed on a Thermo Finnigan LCQ Deca XP ion trap mass spectrometer (ITMS) in positive and negative modes. The nomenclature proposed by Roepstorff and Fohlman [5] was used for the parent and fragment ions.

\section{H.1.3 Fenton Reaction}

SP-B ${ }_{1-25}(500 \mu \mathrm{M})$ was incubated with $600 \mu \mathrm{M} \mathrm{FeCl}_{2}, 600 \mu \mathrm{M}$ sodium EDTA, and $30 \mathrm{mM} \mathrm{H}_{2} \mathrm{O}_{2}$ in $1 \mathrm{~mL}$ water at $37^{\circ} \mathrm{C}$ for 12,18 , and $24 \mathrm{~h}$. The peptide was purified using a Varian C18 OMIX $100 \mu \mathrm{L}$ pipette tip. The sample solution was diluted to an appropriate concentration with 1:1 

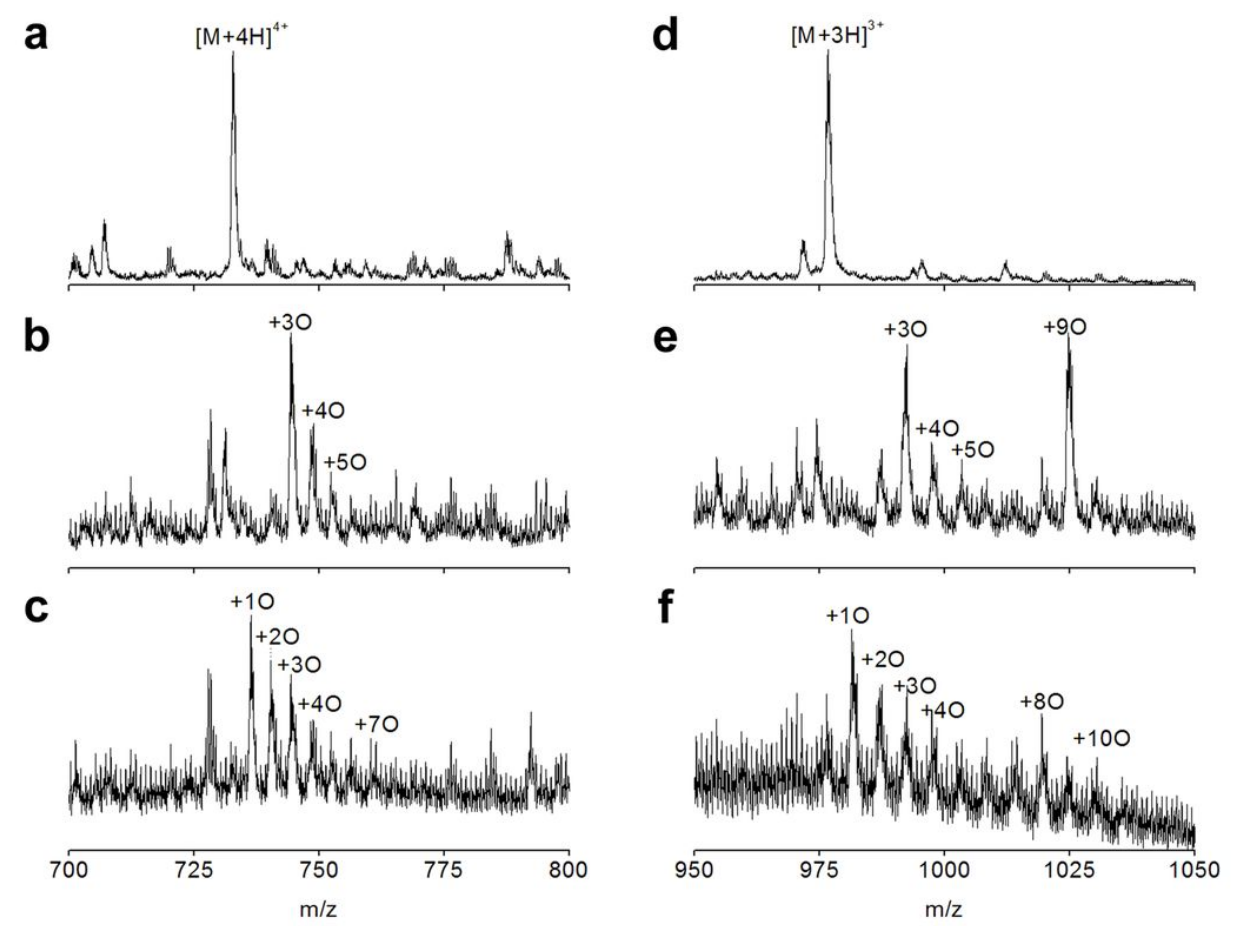

Figure H.1: (a) Quadruply charged SP-B $\mathrm{B}_{1-25}$ and oxidized products from (b) the bulk-phase $\mathrm{O}_{3}$ reaction and (c) the Fenton reaction with intact SP-B ${ }_{1-25}$. (d) Triply charged SP-B ${ }_{1-25}$ and oxidized products from (e) the bulk-phase $\mathrm{O}_{3}$ reaction and (f) the Fenton reaction are also shown.

water/methanol and $1 \%$ acetic acid by volume. Product analysis was performed on a Micromass QTof2 quadrupole time of flight mass spectrometer in the positive ion mode.

\section{H.2 Results and Discussion}

Triply and quadruply protonated SP-B $\mathrm{B}_{1-25}$ are observed as dominating ions in the ESI-MS spectrum with a small amount of doubly protonated peptides (Figures H.1a and H.1d). Figure H.1 shows oxidized products of triply and quadruply charged SP- $\mathrm{B}_{1-25}$ from the bulk-phase $\mathrm{O}_{3}$ reaction and the Fenton reaction with intact SP-B ${ }_{1-25}$. As seen in Figures H.1b and H.1e, incorporation of three and nine oxygens in SP- $\mathrm{B}_{1-25}$ are observed as major products from the bulk-phase $\mathrm{O}_{3}$ reaction with the peptide. Other oxygenated peptides $(+4 \mathrm{O}$ and $+5 \mathrm{O})$ are also observed as minor products in the spectrum. The Fenton reaction yields a series of oxidized SP-B $\mathrm{B}_{1-25}$ products with up to 10 oxygen atoms (Figures H.1c and H.1f). It is notable that the bulk-phase $\mathrm{O}_{3}$ reaction yields dominating products of 3- and 9-oxygenated SP-B ${ }_{1-25}$. This suggests the high stability of SP-B ${ }_{1-25}$ incorporating 3 and 9 oxygen atoms. In order to determine structures of the oxidized SP-B $1-25$ by the bulk-phase $\mathrm{O}_{3}$ reaction, trypsin digest was performed after applying $\mathrm{O}_{3}$ for 3 min to the SP-B ${ }_{1-25}$ solution. 


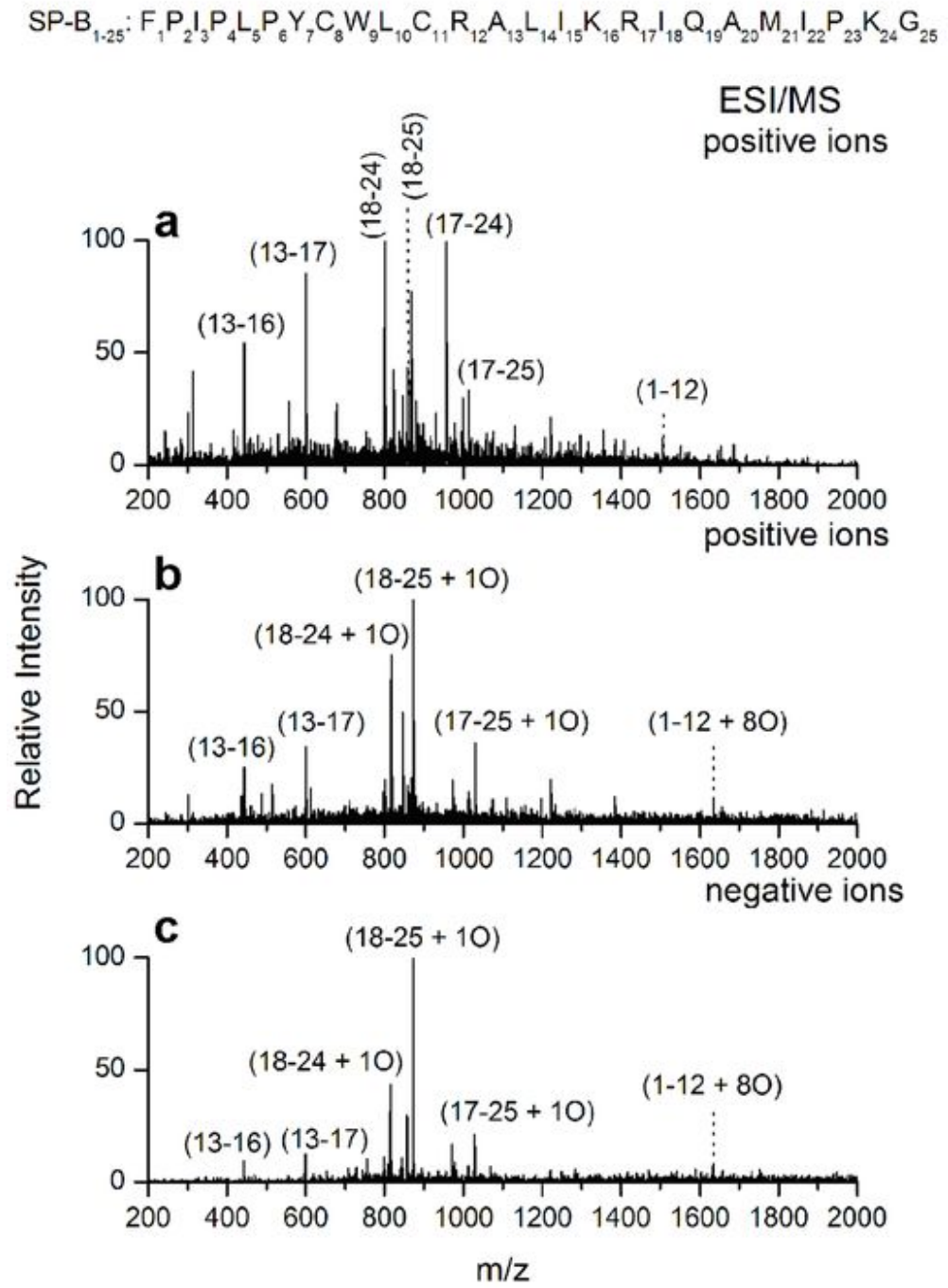

Figure H.2: (a) The ESI mass spectrum of the trypsin digests of SP-B ${ }_{1-25}$ in positive mode. (b) The ESI mass spectrum of the trypsin digests of the oxidized $\mathrm{SP}_{-} \mathrm{B}_{1-25}$ from the bulk-phase $\mathrm{O}_{3}$ reaction in positive mode. (c) The negative mode ESI mass spectrum of the trypsin digests of the oxidized $\mathrm{SP}-\mathrm{B}_{1-25}$ from the bulk-phase $\mathrm{O}_{3}$ reaction. 


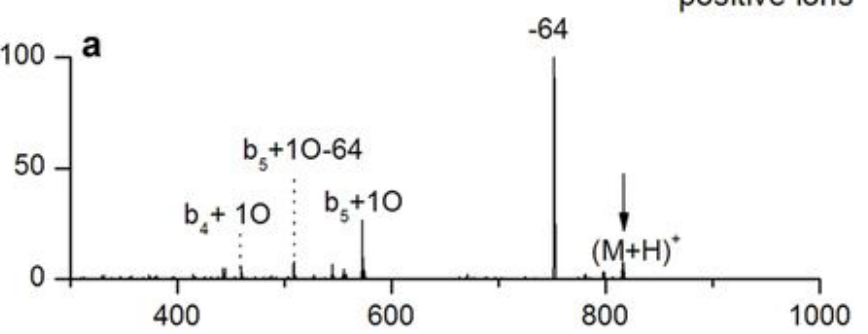

M: FPIPLPYCWLCR (1-12) +80
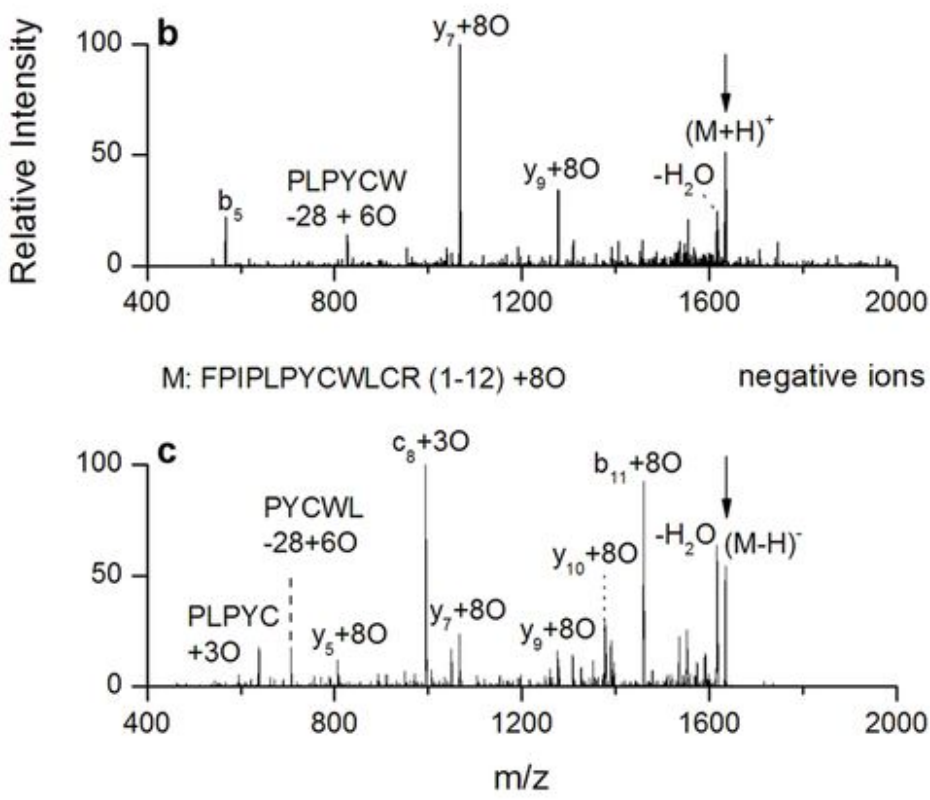

Figure H.3: (a) The CID spectrum of cationic IQAMIPK $+3 \mathrm{O}$ at $\mathrm{m} / \mathrm{z} 817$ from the tryptic digest of the oxidized SP-B ${ }_{1-25}$. (b) The CID of cationic FPIPLPYCWLCR $+8 \mathrm{O}$ at $\mathrm{m} / \mathrm{z} 1636$ from the tryptic digest of the oxidized SP-B ${ }_{1-25}$. (c) The CID of anionic FPIPLPYCWLCR $+8 \mathrm{O}$ at $\mathrm{m} / \mathrm{z}$ 1634 from the tryptic digest of the oxidized SP-B $\mathrm{B}_{1-25}$.

The ESI mass spectrum of a trypsin digest of SP- $\mathrm{B}_{1-25}$ exhibits 7 major ion peaks. The masses and segments of the observed tryptic digest ions of SP-B ${ }_{1-25}$ are indicated in Figure H.2a. The ESI mass spectrum of a trypsin digest of SP- $\mathrm{B}_{1-25}$ oxidized by reaction with bulk-phase $\mathrm{O}_{3}$ exhibits segments with MetSO (m/z 817, $\mathrm{m} / \mathrm{z} 874$, and $\mathrm{m} / \mathrm{z}$ 1030) and the less abundant N-terminal segment of FPIPLPYCWLCR $+8 \mathrm{O}$ at m/z 1636 in positive ion mode (Figure H.2b). The negative ion mode ESI mass spectrum also exhibits corresponding deprotonated segment ion peaks (Figure H.2c).

The structures of segments are confirmed by CID spectra. As seen in Figure H.3a, the CID of the ion at $\mathrm{m} / \mathrm{z} 817$ exhibits the elimination of hydrosulfinylmethane $\left(\mathrm{CH}_{4} \mathrm{SO}, 64\right.$ mass unit), which is the characteristic dissociation pathway of methionine sulfoxide (MetSO) [6, 7]. The structure of the segment FPIPLPYCWLCR $+8 \mathrm{O}$ is also investigated using CID. Fragments from the CID of 


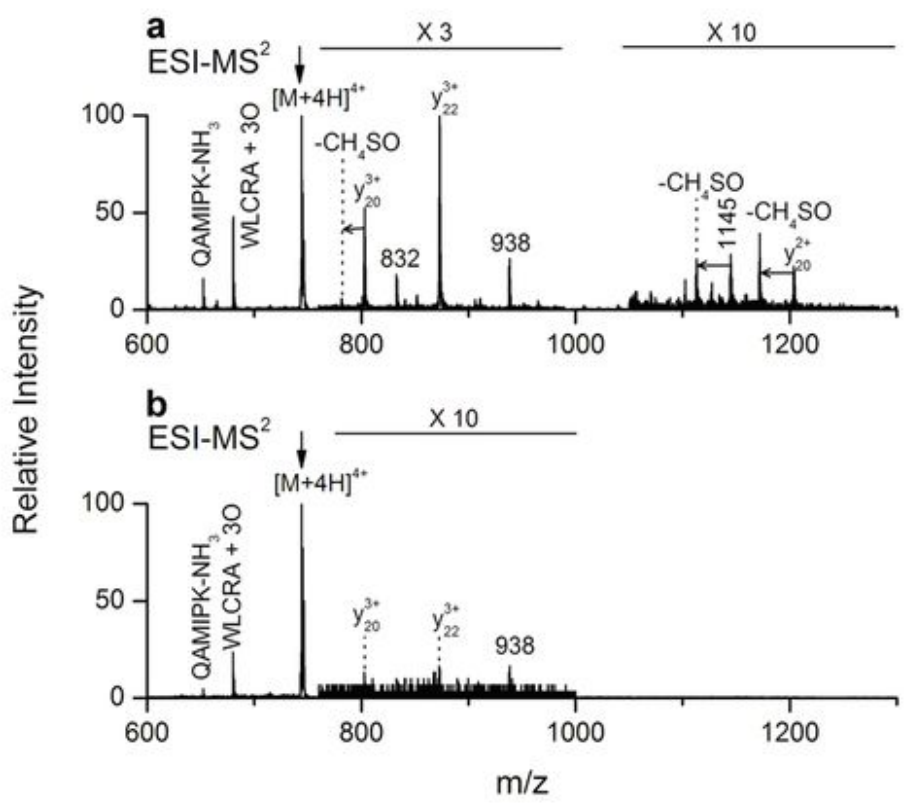

Figure H.4: ESI-MS ${ }^{2}$ of triply oxygenated products of quadruply protonated SP-B ${ }_{1-25}$ from (a) bulk-phase $\mathrm{O}_{3}$ application, and from (b) the Fenton reaction. M denotes a parent ion, which is triply oxygenated $\mathrm{SP}-\mathrm{B}_{1-25}$.

the cationic FPIPLPYCWLCR +80 at $\mathrm{m} / \mathrm{z} 1636$ yields $y$-type fragments $\left(y_{7}\right.$ and $\left.y_{9}\right)$ with all eight oxygen atoms as dominant products (Figure H.3b). The fragment PLPYCW with six oxygen atoms indicates that $\mathrm{Cys}_{8}$ and $\mathrm{Trp}_{9}$ are oxidized to sulfonic acid $(+3 \mathrm{O})$ and hydroxy-Nformylkynurenine (HNFKyn, $+3 \mathrm{O})$, respectively. Thus, we can assume that $\mathrm{Cys}_{11}$ is oxidized to sulfenic acid (+ $2 \mathrm{O})$. The structures are further confirmed by the fragments of $\mathrm{c}_{8}+3 \mathrm{O}$ and PLPYC $+3 \mathrm{O}$ resulting from the CID of FPIPLPYCWLCR $+8 \mathrm{O}$ in negative ion mode (Figure H.3c). It is notable that $\mathrm{Cys}_{8}$ and $\mathrm{Cys}_{11}$ are located next to each other in the helical structure [8]. Asymmetric oxidation of closely located Cys residues is caused by the possible formation of sulfonic anhydride $\left(\mathrm{R}_{1}-\mathrm{O}_{2} \mathrm{SOSO}_{2}-\right.$ $R_{2}$ ). No segment peak is observed from the triply oxygenated $S P-B_{1-25}$ after tryptic digest of the products from the bulk-phase $\mathrm{O}_{3}$ reaction.

The observed high abundance of SP-B $\mathrm{B}_{1-25}$ where 3 and 9 oxygen atoms have been added can be explained by the distinct oxidation mechanisms of $\mathrm{O}_{3}$ in aqueous solution. Cys easily undergoes oxidation by $\mathrm{O}_{3}$ to form sulfonic acid in aqueous solution primarily by reactions with secondary oxidants [9]. Cys8 and $\mathrm{Cys}_{11}$, which are located near the hydrophobic N-terminal site in SP-B ${ }_{1-25}$, are hardly oxidized by reactive oxygen species (ROS) at the air-liquid interface. Trp 9 and $\operatorname{Met}_{21}$ are oxidized to form NFKyn and MetSO by ozonolysis and ROS, respectively, by interfacial reactions. This induces a change in peptide orientation in the interface that results in NFKyn, Cys 8 , and Cys $_{11}$ being exposed to ROS for further oxidation. As a result, NFKyn occurs to form HNFKyn and, simultaneously, $\mathrm{Cys}_{8}$ and $\mathrm{Cys}_{11}$ are also oxidized by ROS to form sulfonic anhydride. 
The SP- $\mathrm{B}_{1-25}$ products with three additional oxygen atoms from both reactions are analyzed using CID. Figure H.4 shows CID spectra (ESI-MS²) of triply oxygenated products of quadruply protonated $\mathrm{SP}_{-} \mathrm{B}_{1-25}$ from bulk-phase $\mathrm{O}_{3}$ application and from the Fenton reaction. The ESI-MS ${ }^{2}$ spectrum of the triply oxygenated $\mathrm{SP}-\mathrm{B}_{1-25}$ by bulk-phase $\mathrm{O}_{3}$ application implies the presence of two products, one with NFKyn $(+2 \mathrm{O})$ and MetSO $(+1 \mathrm{O})$, and the other with HNFKyn $(+3 \mathrm{O})$. Evidence of the MetSO in the oxidized SP-B ${ }_{1-25}$ is found from the CID experiment involving the quadruply charged SP-B $\mathrm{B}_{1-25}$ product from the bulk-phase $\mathrm{O}_{3}$ reaction (Figure H.4a). For example, the paired fragments of $y_{20}-\mathrm{CH}_{4} \mathrm{SO}$ and $y_{20}$ fragments, which are observed at $\mathrm{m} / \mathrm{z} 782$ and $\mathrm{m} / \mathrm{z} 803$ (triply charged) and at m/z 1172 and m/z 1204 (doubly charged), confirm the presence of MetSO (Figure H.4a). Interesting CID fragments are also observed in the ESI-MS ${ }^{2}$ spectrum. The singly charged fragment at $\mathrm{m} / \mathrm{z} 680, \mathrm{~m} / \mathrm{z} 832$, and $\mathrm{m} / \mathrm{z} 938$ are WLCRA $+3 \mathrm{O}$, LPYCWLCRALIKR $+3 \mathrm{O}$ and PLPYCWLCRALIKRI $+3 \mathrm{O}$, respectively. These fragments are evidence of the formation of HNFKyn through the oxidation of $\operatorname{Trp}_{9}$ from the bulk-phase $\mathrm{O}_{3}$ reaction. This is further supported by the singly charged fragment at $\mathrm{m} / \mathrm{z} 652$, QAMIPK- $\mathrm{NH}_{3}$, which indicates that no oxidation has occurred at Met $_{21}$. The ESI-MS ${ }^{2}$ spectrum of the triply oxygenated SP-B $\mathrm{B}_{1-25}$ by bulk-phase $\mathrm{O}_{3}$ reaction implies the presence of two products, one with NFKyn $(+2 \mathrm{O})$ and MetSO $(+1 \mathrm{O})$, and the other with HNFKyn $(+3 \mathrm{O})$. The CID fragment abundance implies that the bulk-phase $\mathrm{O}_{3}$ reaction with $\mathrm{SP}_{-} \mathrm{B}_{1-25}$ yields more product peptides with HNFKyn compared to product peptides with NFKyn and MetSO. The ESI-MS ${ }^{2}$ spectrum of SP- $\mathrm{B}_{1-25}$ product from the Fenton reaction is shown in Figure H.4b. The CID of the Fenton product exhibits the presence of HNFKyn at m/z 652, $\mathrm{m} / \mathrm{z} 680$, and $\mathrm{m} / \mathrm{z}$ 938, which corresponds to the singly charged fragments QAMIPK- $\mathrm{NH}_{3}$, WLCRA $+3 \mathrm{O}$, and PLPYCWLCRALIKRI $+3 \mathrm{O}$, respectively. However, evidence for the formation of the product with NFKyn and MetSO is not found in the spectrum. 


\section{Bibliography}

[1] Binder, K. Rep. Prog. Phys. 1997, 60, 487-559.

[2] Candra, P. M.S. Dissertation, University of Minnesota, 2006.

[3] Fixman, M. Proc. Natl. Acad. Sci. U.S.A. 1974, 71, 3050-3053.

[4] Karagulian, F.; Lea, A. S.; Dilbeck, C. W.; Finlayson-Pitts, B. J. Phys. Chem. Chem. Phys. 2008, 10, 528-541.

[5] Roepstorff, P.; Fohlman, J. Biomedical Mass Spectrometry 1984, 11, 601-601.

[6] Clauser, K. R.; Hall, S. C.; Smith, D. M.; Webb, J. W.; Andrews, L. E.; Tran, H. M.; Epstein, L. B.; Burlingame, A. L. Proc. Natl. Acad. Sci. U. S. A. 1995, 92, 5072-5076.

[7] Qin, J.; Chait, B. T. Anal. Chem. 1997, 69, 4002-4009.

[8] Bruni, R.; Taeusch, H. W.; Waring, A. J. Proc. Natl. Acad. Sci. U. S. A. 1991, 88, 7451-7455.

[9] Berlett, B. S.; Stadtman, E. R. J. Biol. Chem. 1997, 272, 20313-20316. 